Seton Hall University eRepository@Seton Hall

Seton Hall University Dissertations and Theses (ETDs)

Seton Hall University Dissertations and Theses

Winter 12-2000

\title{
Dendritic Polymers as Bonded Stationary Phases in Capillary Electrochromatography
}

Chiayu Helen Chao

Seton Hall University

Follow this and additional works at: https://scholarship.shu.edu/dissertations

Part of the Polymer Chemistry Commons

\section{Recommended Citation}

Chao, Chiayu Helen, "Dendritic Polymers as Bonded Stationary Phases in Capillary Electrochromatography" (2000). Seton Hall University Dissertations and Theses (ETDs). 1253.

https://scholarship.shu.edu/dissertations/1253 


\title{
Dendritic Polymers as Bonded Stationary Phases in
}

\section{Capillary Electrochromatography}

\author{
Thesis by \\ Chiayu Helen Chao \\ In Partial Fulfilment of the Requirements \\ for the Degree of \\ Doctor of Philosophy
}

Seton Hall University

South Orange, New Jersey 07079

December, 2000 
We certify that we have read this thesis and that in our opinion it is adequate in scientific scope and quality as a dissertation for the degree of Doctor of Philosophy.

\section{Approved}

Aamer i. Itanow

James. E. Hanson, Ph. D.

Research Mentor

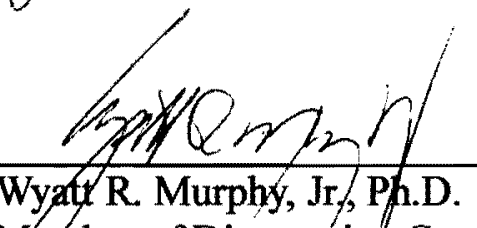

Member of Dissertation Committee
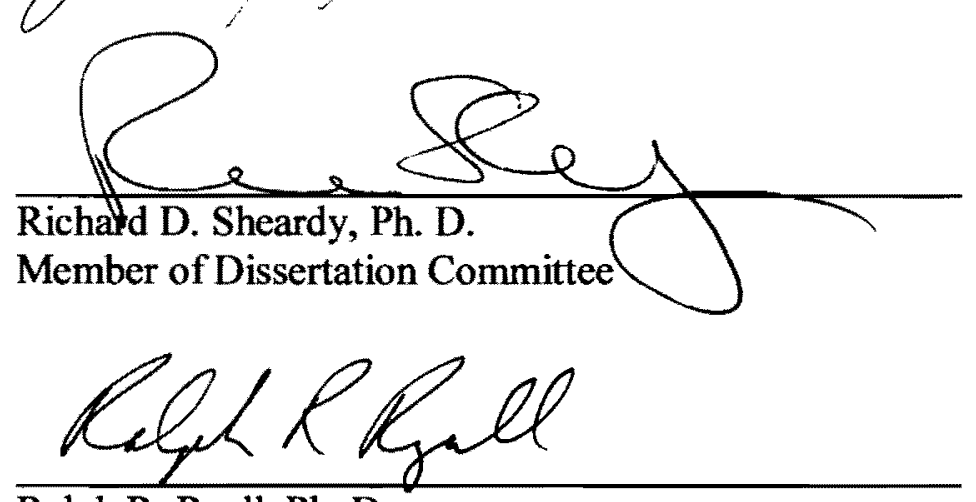

Ralph R. Ryall, Ph. D.

Member of Dissertation Committee

Director, New Product Research - Analytical Development

The R. W. Johnson Pharmaceutical Research Institute

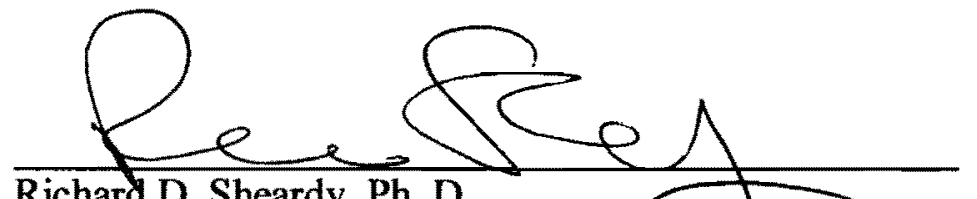

Richard D. Sheardy, Ph. D.

Approved for the Chemistry Departmext

Chairperson, Department of Chemistry

Seton Hall University 
(C) 2000

Helen Chia-yu Chao

All Rights Reserved 


\section{DEDICATION}

To my two sons, Hanvey and Hansun. without their spiritual support, their many years of sacrifice and their confidence in me, I would not have had the courage to complete my studies.

This thesis is also dedicated to my mentor at The R. W. Johnson Pharmaceutical Research Institute, Chuck Shaw, who sacrificed his personal time to coach me in all the spectroscopy work. Without his encouragement and guidance, this work would not have been completed.

I would also like to dedicate this work to my late parents, they supported me every decision I made throughout my life. By deviating from the normal Chinese tradition, they always encouraged me to continue my education. 


\section{Acknowledgements}

I would like to express my gratitude to my thesis advisor, Dr. James Hanson, for all of his time, assistance, and guidance during the course of my doctoral research at Seton Hall University. Because of his enthusiasm, support and guidance throughout my research, I was able to complete my doctoral work at this institution. Likewise, I wish to convey my sincere appreciation for my mentor at The R. W. Johnson Pharmaceutical Research Institute (RWJ-PRI), Chuck Shaw, and the countless valuable contributions he has provided towards the achievement of my research goals. I also wish to thank Dr. Wyatt R. Murphy Jr. for his helpful suggestions and friendly discussions during my residency at Seton Hall University.

I am very grateful to my dissertation reader, Dr. Wyatt R. Murphy Jr., Dr Richard D. Sheardy and Dr. Ralphy R. Ryall for their valuable suggestions and time. I also wish to thank all my labmates and friends, Kate Jensen, Jeanne M. Riley, Biaming Xiao, Patrick Hanlon, Richard Allston, Alfredo Mellace, Hye-Jung Han, Sueyuan Li, and Qiuxia Zhao for their friendship and encouragement at different moments during my stay at Seton Hall. I would like to acknowledge some of my additional friends: Amal Yamany, Lorna Waters, Rosemary Wills and Lee Craven for their endless encouragement and confidence in me.

The author also wishes to thank The R. W. Johnson Pharmaceutical Research Institute management board and the scholarship program for funding my studies. Without their financial support, I would never have been able to fulfill my life long dream. 


\begin{abstract}
Dendrimers are an interesting new class of polymers. Their unusual chemical and physical properties have great potential in many applications. Polyamidoamine starburst dendrimers (PAMAM-SBDs) have shown promising results as a pseudostationary phase in Micellar Electrokinetic Capillary Chromatography (MECC). However, few chromatographic applications have been explored using dendrimers. The objective of our study is to explore the potential of using poly(aryl ether) dendritic polymers as a bonded stationary phase in open tubular Capillary Electrochromatography (CEC).

The syntheses of the first generation (G1) and second generation (G2) of poly(aryl ether) alcohols have been achieved in this study. The dendritic polymers can be chemically bonded onto the inner wall of a fused silica capillary column by using 3(triethoxysilyl)propyl isocyanate as an anchoring agent. Four different bonded phases were studied: the G1 and G2 poly(aryl ether) dendrimers, a C18 alkyl chain, and 4pyridylcarbinol. Model separations of different analytes were examined over a range of $\mathrm{pH}$ values with a variety of buffer electrolytes.

The suppression in electroosmotic flow (EOF) was observed using thiourea as a neutral marker. A decrease in EOF with G1 coating versus a C18 coating was obtained over the $\mathrm{pH}$ range $4-9.3$. The EOF was completely suppressed when the capillary was coated with second generation of poly(aryl ether) carbamate. The permanent surface modification method in this report proved to be stable over the $\mathrm{pH}$ range of $4.0-9.3$. Improved selectivity and retention were observed with polyaromatic and basic compounds.
\end{abstract}


Table of Contents

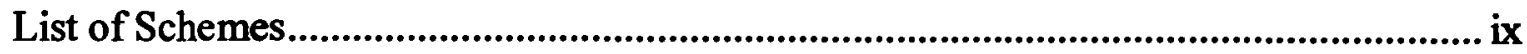

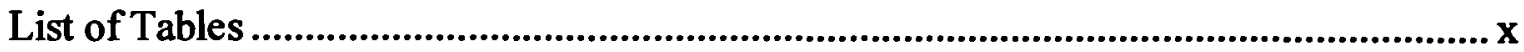

List of Figures......................................................................................................

CHAPTER 1 Introduction

$1.1 \quad$ Introduction............................................................................................................ 1

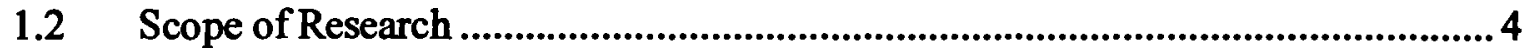

1.3 Outline of dissertation.......................................................................................... 6

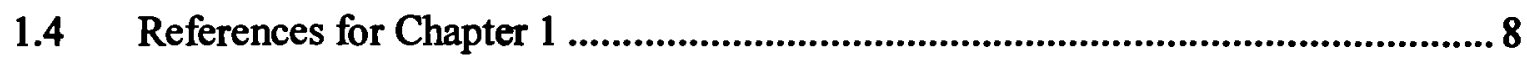

CHAPTER 2 Capillary Electrochromatography

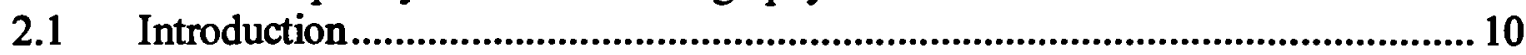

2.2 Capillary electrophoresis ........................................................................................ 10

2.2.1 Modes of capillary electrophoresis .............................................................. 13

2.2.2 Electroosmotic flow ......................................................................... 15

2.2.3 Theory of CZE operation .................................................................... 20

2.3 Capillary Electrochromatography (CEC) ........................................................... 28

2.3.1 Electroosmotic pumping29

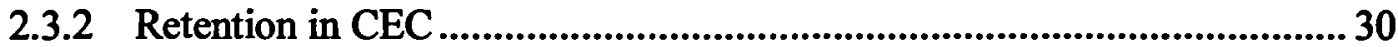

2.3.3 Separation efficiency in CEC ............................................................... 32

2.3.4 Separation efficiency in OT-CEC ........................................................33

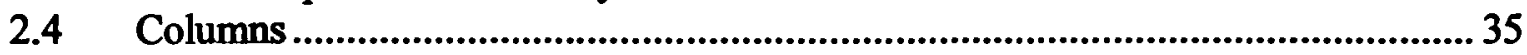

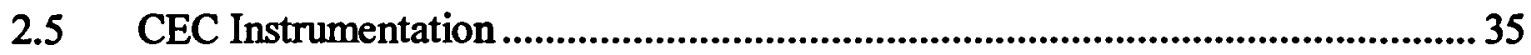

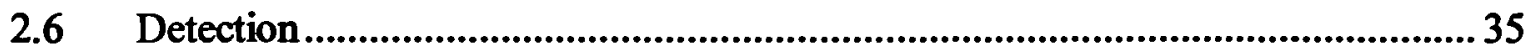

2.7 Advantages and Limitations of CEC ............................................................36

2.7.1 Advantages of CEC...............................................................................36

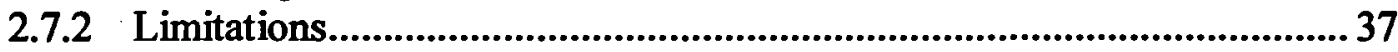

$2.8 \quad$ References for Chapter 2 ................................................................................... 39

CHAPTER 3 Dendrimers

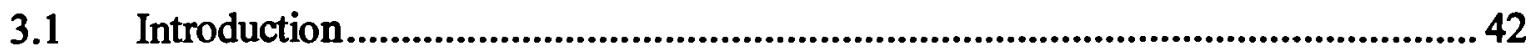

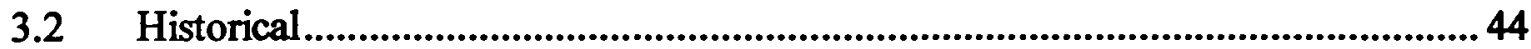

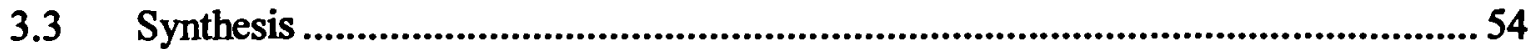

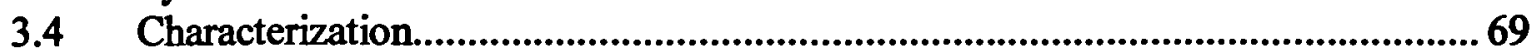

3.5 Physical behavior of dendritic molecules ..................................................... 71

3.5.1 Localization of end groups in dendrimers................................................... 71

3.5.2 Dendrimers versus linear macromolecules................................................ 73

3.5.3 The behavior of dendrimers on surfaces ............................................... 74

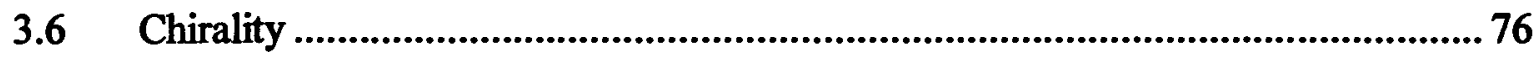

$3.7 \quad$ References for Chapter 3 ................................................................................. 80 
CHAPTER 4 Dendrimer Synthesis

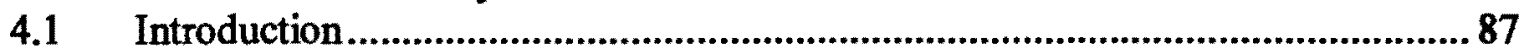

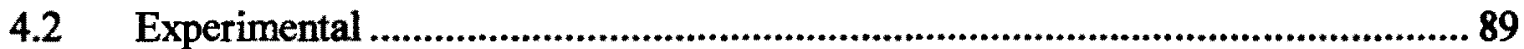

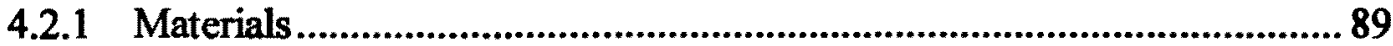

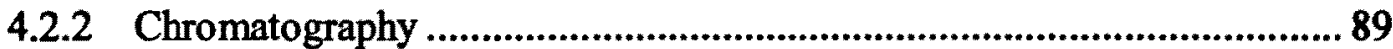

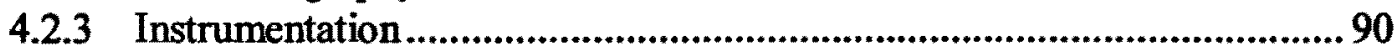

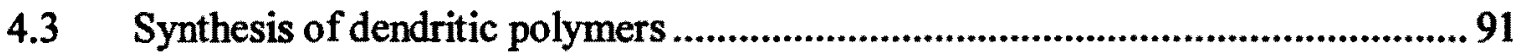

4.3.1 Methyl 3,5-dihydroxybenzoate (1) ........................................................92

4.3.2 3,5-dibenzyloxybenzyl alcohol (2) ......................................................... 93

4.3.3 3,5-dibenzyloxybenzyl methane sulfonate (3) ........................................94

4.3.4 Methyl 3,5-(3',5'-benzyloxybenzyloxy)benzoate (4) ..............................95

4.3.5 3,5-(3',5'-benzyloxybenzyloxy)benzyl alcohol (5) ................................96

4.4 Synthesis of column coating materials ................................................................97

4.4.1 General procedure...........................................................................98

4.4.2 3,5-dibenzyloxybenzyl 3-(triethoxysilyl)propyl carbamate (9), $\mathrm{G}_{1}$

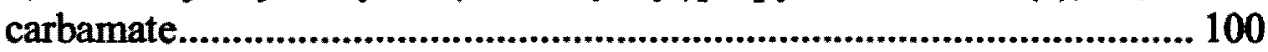

4.4.3 3,5-(3',5'-benzyloxybenzyloxy)benzyl 3-(triethoxysilyl)propyl

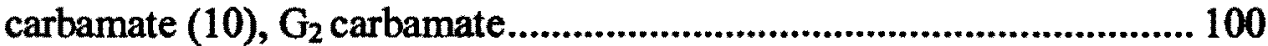

4.4.4 1-Octadecyl 3-(triethoxysilyl)propyl carbamate (11), stearyl

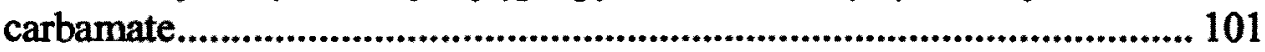

4.4.5 4-pyridyl carbinol 3-(triethoxysilyl)propyl carbamate (12) .................... 101

4.4.6 Benzyl 3-(triethoxysilyl)propyl carbamate (13) ..................................... 102

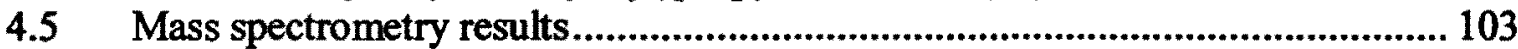

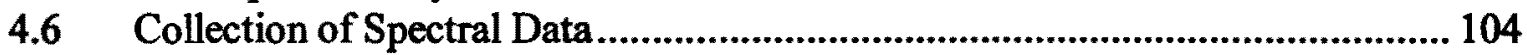

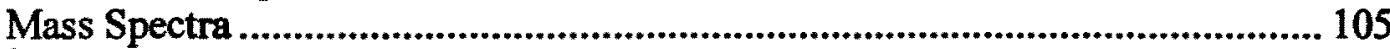

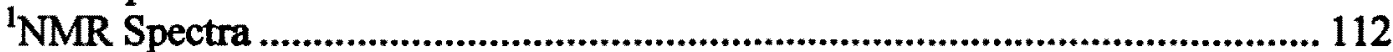

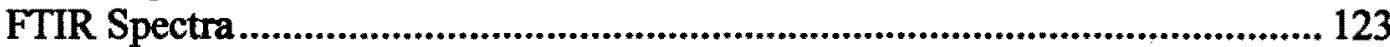

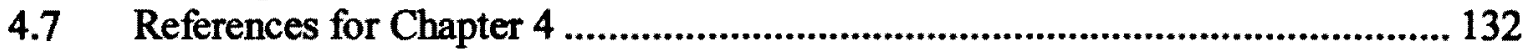

CHAPTER 5 Dendrimer Coated Capillary Columns in OT-CEC

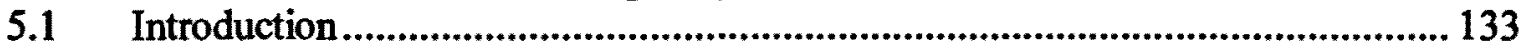

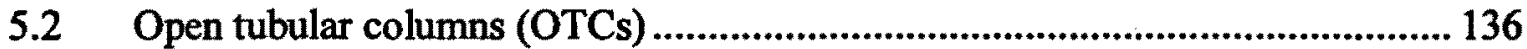

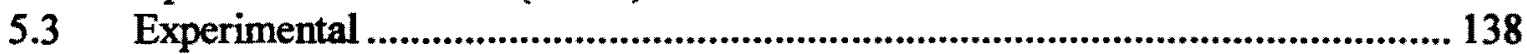

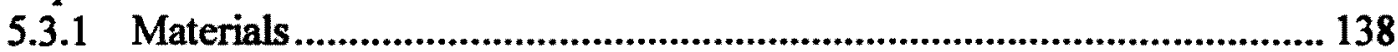

5.3.2 Column coatings .................................................................................... 138

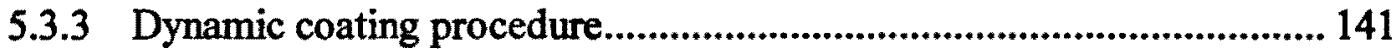

5.3.4 Effects of dendritic polymers coating ................................................... 141

5.3.5 Effects of dendritic polymers on analytes ............................................ 143

5.3.6 Effects of Si-O-C versus Si-O-Si bonds............................................... 144

5.3.7 Permanent coating using silane compound as an anchoring agent......... 149

5.3.8 Preparation of the coated capillary column ............................................ 150

5.4 Surface characterization of the bonded stationary phases .................................. 153

5.4.1 Preparation of dendrimer coatings on fused silica plates........................ 153

5.4.2 Spectroscopic results ................................................................................ 154

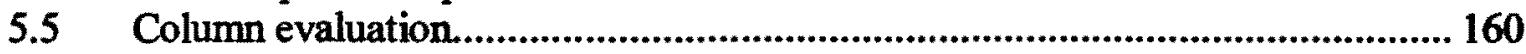

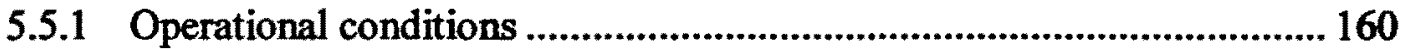




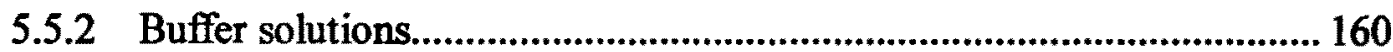

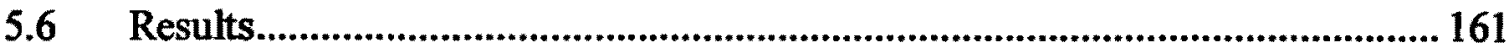

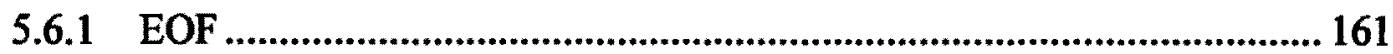

5.6.2 Evaluation of coated capillaries with polyaromatic compounds ............. 161

5.6.3 Evaluation of coated capillaries with basic amine substances ................ 162

5.6.4 Evaluation of coated capillaries with basic proteins............................... 163

5.6.5 Evaluation of coated capillaries with anions ............................................ 163

5.6.6 Evaluation of coated capillaries with structural isomers.......................... 164

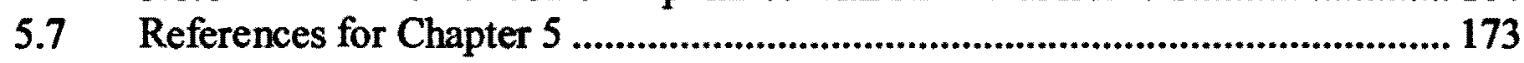

CHAPTER 6 Discussion and Conclusion

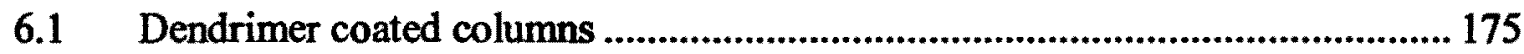

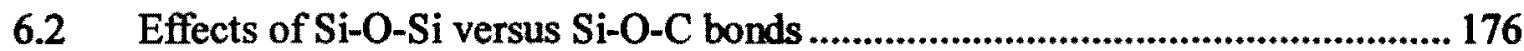

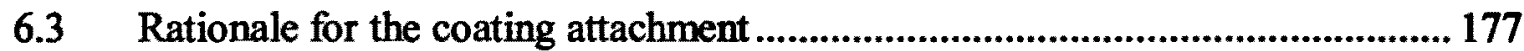

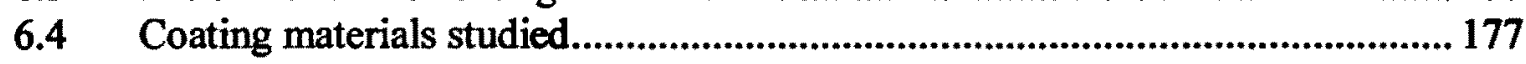

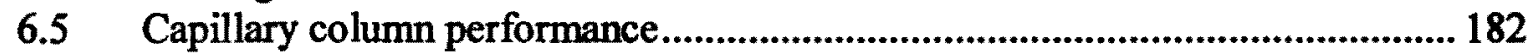

6.5.1 Evaluation of coated capillaries with polyaromatic compounds ............. 182

6.5.2 Evaluation of coated capillaries with basic amine substances .................. 186

6.5.3 Evaluation of coated capillaries with basic proteins.................................. 186

6.5.4 Evaluation of coated capillaries with anions ............................................. 186

6.5.5 Evaluation of selectivity effect on structural isomers.............................. 187

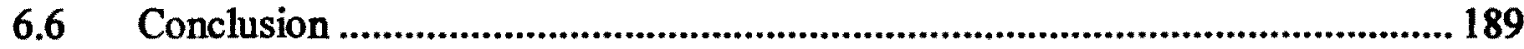






\section{List of Schemes}

Scheme 3-1. Tomalia's schematical representation of the divergent dendrimer growth.

Scheme 3-2. A) Divergent preparation of dendrimer, B) Convergent preparation of dendrimer.

Scheme 3-3. Synthetic scheme for starburst PAMAM dendrimers. ............................... 56

Scheme 3-4. Convergent preparation of poly(aryl ether) dendron possessing a single nitrile terminus.................................................................................5

Scheme 3-5. Synthetic scheme using the CDI chemistry............................................58

Scheme 3-6. The double exponential growth process......................................................59

Scheme 3-7. Synthetic scheme illustrates Fréchet's $\mathrm{AB}_{2}$ growth process. ....................61 


\section{List of Tables}

Chapter 4 Dendrimer synthesis

Table 4-1. LC/MS Electrospray Ionization Data for the Compounds

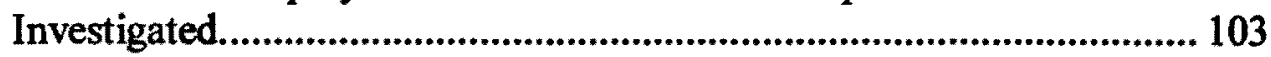

Chapter 5 Dendrimer Coated Capillary Columns in OT-CEC

Table 5-1. Summary of apparent mobility observed with dynamic coating process.

Table 5-2. Resolution comparison between the Si-O-C and Si-O-Si coatings. ....... 149

Table 5-3. The experimental EOF values at different $\mathrm{pH}$ using thiourea as a Neutral marker. 165

Table 5-4. The migration time of 2'-fluoroacetanilide........................................... 165

Chapter 6 Discussion and Conclusion

Table 6-1. Pyrrolopyridine separation factors obtained from G1, C18 coated and uncoated columns. 


\section{List of Figures}

Chapter 1 Introduction

Figure 1-1. Intrinsic viscosity behavior of polyether dendrimers and polystyrene. .......3

Figure 1-2. Structural and topological relationships between (a) branched polymers;

(b) $\mu$-gels; (c) macroscopic networks; (d) functionalized $\mu$-gels; and

(e) cascade polymers.

Figure 1-3. An example of a small poly (aryl ether) monodendron based on the work of Fréchet.................................................................................................. 5

Figure 1-4. Chemical structure of the five coating materials. ........................................

Chapter 2 Capillary Electrochromatography

Figure 2-1. Diagram of a basic capillary electrophoresis system............................... 13

Figure 2-2. Representation of the double-layer at the capillary wall........................... 17

Figure 2-3. Differential solute migration superimposed on electroosmotic flow in capillary zone electrophoresis.................................................................. 18

Figure 2-4. Effect of $\mathrm{pH}$ on electroosmotic flow mobility in various capillary materials.

Figure 2-5. Electroosmotic flow mobility as a function of buffer concentration and ionic strength.

Figure 2-6. Elution order for cations ( + ), nonionics ( 0$)$, and anions $(-)$ based on the cumulative effects of electrophoresis and strong electroosmotic flow toward the cathode.

Figure 2-7. Flow profile in a capillary tube with (a) electro-drive, (b) pressure drive.

Chapter 3 Dendrimers

Figure 3-1. Dendritic box hosts the guest molecules in its cavities.............................. 46

Figure 3-2. Supramolecular arrangement to form a hexameric dendrimer.................. 47 
Figure 3-3. Starburst dendrimer, poly(propylene imine) dendrimers. 48

Figure 3-4. Arborol dendrimer, 27 cascade: hexane[3-1,1,1]: (4-oxapentylidene) : (3-oxo 2-azapropylidene): methanol.

Figure 3-5. Schematic representation of a backfold trifunctional fifth generation dendrimer according to Boris and Rubinstein.

Figure 3-6. Dendritic copolymer responds to changes in its environment...................53

Figure 3-7. Liao and Moss's organoruthenium dendrimers. 65

Figure 3-8. The molecular encapsulation principle: closing and opening of a dendrimer box.

Figure 3-9. The synthesis of PPI dendrimers (reactions A and B) and alternative, unwanted reaction paths $\mathrm{C}$ and $\mathrm{D}$.

Figure 3-10. Unwanted reactions in the PAMAM-synthesis: retro-Michael reaction (A) and Lactam formations (B).

Figure 3-11. Structure of a Fréchet-type polyaryl ether dendritic wedge.

Figure 3-12. Schematic representation of the different modes of adsorption of dendrimers on surfaces.

Figure 3-13. A "phase diagram" that shows the shape of dendrimers in adsorbed monolayers depends on the strength of the adsorption interaction and the dendrimer generation. 74

Figure 3-14. Schematic representations of different types of chiral dendrimers. 75

Figure 3-15. The first example of a "grafted" dendrimer prepared by Newkome et al. 76

Figure 3-16. Chiral dendrimers with a core chirality center. 77

Figure 3-17. Examples of chiral cores that can potentially be utilized for the generation of chiral dendrimers.

Chapter 4 Dendrimer Synthesis

Figure 4-1. Synthetic scheme illustrating the mesylation route. 88 
Figure 4-2. Chemical structures of the poly(aryl ether) dendrimers and the process intermediates.

Figure 4-3. The chemical structures of the desired poly(benzyl ether) dendrimers and other alcohol materials.

Figure 4-4. Synthesis scheme illustrating the preparation of carbamates from the coupling reaction.

Figure 4-5. The chemical structures of the carbamates used as coating materials for CEC evaluation.

Figure 4-6. Mass spectrum of methyl 3, 5-dihydroxybenzoate (1). 106

Figure 4-7. Mass spectrum of 3, 5-dibenzyloxybenzylalcohol (2). 107

Figure 4-8. Mass spectrum of 3, 5-dibenzyloxy benzyl methane sulphonate (3)...... 108

Figure 4-9. Mass spectrum of 3, 5-(3', 5'-benzyloxybenzyloxy) benzyl alcohol (5).

Figure 4-10. Mass spectrum of 4-pyridyl carbinol 3-(triethoxysilyl)propyl carbamate (12).

Figure 4-11. Mass spectrum of Benzyl 3-(triethoxysilyl)propyl carbamate (13)........ 111

Figure 4-12. $\quad 300-\mathrm{MHz}{ }^{\mathrm{I}} \mathrm{H}$ NMR spectrum of methyl 3, 5-dihydroxybenzoate (1).... 113

Figure 4-13 300-MHz ${ }^{1} \mathrm{H}$ NMR spectrum of 3, 5-dibenzyloxybenzylalcohol (2). .... 114

Figure 4-14. 300-MHz ${ }^{1} \mathrm{H}$ NMR spectrum of 3, 5-dibenzyloxy benzyl methane sulphonate (3).

Figure 4-15. 300-MHz 'H NMR spectrum of Methyl 3, 5-(3', 5'-benzyloxybenzyloxy) benzoate (4). 116

Figure 4-16. 300-MHz ${ }^{1} \mathrm{H}$ NMR spectrum of 3, 5-(3', 5'-benzyloxybenzyloxy) benzyl alcohol (5).

Figure 4-17. 300-MHz ${ }^{1} \mathrm{H}$ NMR spectrum of 3, 5-dibenzyloxybenzyl 3-(triethoxysilyl)propyl carbamate (9), G1 carbamate. 118

Figure 4-18. 300-MHz ${ }^{\mathrm{I}} \mathrm{H}$ NMR spectrum of 3, 5-(3', 5'-benzyloxybenzyloxy) benzyl 3-(triethoxysilyl)propyl carbamate (10), G2 carbamate. 119 
Figure 4-19. 300-MHz ${ }^{1} \mathrm{H}$ NMR spectrum of 1-Octadecyl 3-(triethoxysilyl)propyl carbamate (11), Stearyl Carbamate.

Figure 4-20. 300-MHz ${ }^{1} \mathrm{H}$ NMR spectrum of 4-pyridyl carbinol 3-(triethoxysilyl)propyl carbamate (12).

Figure 4-21. 300-MHz ${ }^{1} \mathrm{H}$ NMR spectrum of Benzyl 3-(triethoxysilyl)propyl carbamate (13). 122

Figure 4-22. FTIR spectrum of methyl 3, 5-dihydroxybenzoate (1)......................... 124

Figure 4-23. FTIR spectrum of 3, 5-dibenzyloxybenzylalcohol (2).......................... 125

Figure 4-24. FTIR spectrum of 3, 5-dibenzyloxy benzyl methane sulphonate (3)...... 126

Figure 4-25. FTIR spectrum of Methyl 3, 5-(3', 5'-benzyloxybenzyloxy) benzoate (4).

Figure 4-26. FTIR spectrum of 3, 5-(3', 5'-benzyloxybenzyloxy) benzyl alcohol (5). 128

Figure 4-27. FTIR spectrum of 3, 5-dibenzyloxybenzyl 3-(triethoxysilyl)propyl carbamate (9), G1 carbamate.

Figure 4-28. FTIR spectrum of 4-pyridyl carbinol 3-(triethoxysilyl)propyl carbamate (12). 130

Figure 4-29. FTIR spectrum of benzyl 3-(triethoxysilyl)propyl carbamate (13)........ 131

Chapter 5 Dendrimer coated Capillary Columns in OT-CEC

Figure 5-1. The structure formula of PAMAM, $4^{\text {th }}$ generation. 134

Figure 5-2. The structural formula of a poly(aryl ether) dendrimer, $4^{\text {th }}$ generation. 135

Figure 5-3. Si-O-C bond with G0 from the bromide............................................. 139

Figure 5-4. $\mathrm{Si}-\mathrm{O}-\mathrm{C}$ bond of poly(aryl ether) dendrimer. ........................................ 140

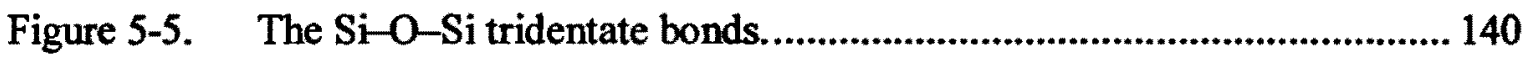

Figure 5-6. Anion test mixture separation comparison with a uncoated column, benzyl bromide coated column, and a G1 poly(aryl ether) coated column. 
Figure 5-7. Anion electropherograms obtained with uncoated and G1 coated capillary columns.

Figure 5-8. Neutral compound electropherograms obtained from uncoated and G1 coated capillary columns.

Figure 5-9. Column coating comparison when benzyl bromide and benzyl triethoxysilane are used.

Figure 5-10. The chemical structure of the five coating materials used for OT-CEC.

Figure 5-11. Diagram of the capillary coating device.

Figure 5-12. FTIR spectra comparison of the Gl and 4-pyridyl carbinol carbamate coated fused silica plates versus an uncoated fused silica plate.

Figure 5-13. UV spectra comparison of the G1 and 4-pyridyl carbinol carbamate coated fused silica discs versus an uncoated silica disc.

Figure 5-14. Raman spectra comparison of the G1 and 4-pyridyl carbinol carbamate coated fused silica plates.

Figure 5-15. Raman spectrum of G1 coated fused silica plate, zoom scan for the wavelength region of $2000 \mathrm{~cm}^{-1}$ to $1800 \mathrm{~cm}^{-1}$.

Figure 5-16. Raman spectrum of 4-pyridyl carbinol carbamate coated fused silica plate, zoom scan for the wavelength region of $2000 \mathrm{~cm}^{-1}$ to $1800 \mathrm{~cm}^{-1}$.

Figure 5-17. The separation of toluene, naphthalene and phenanthrene, obtained with G1 coated capillary column.

Figure 5-18. Electropherograms for the mixture of the phenyl ethylamine, 2, 6-difluoroaniline and 2'-fluoroacetanilide

Figure 5-19. Electropherograms of the protein mixture. 168

Figure 5-20. Anion test mixture electropherograms migration order. 169

Figure 5-21. Epinephrine electropherograms obtained using uncoated, 4-pyridyl carbinol, C18 and G1 coated capillary columns. 170

Figure 5-22. Epinephrine electropherogram obtained at different pHs using a G1 coated column. 
Figure 5-23. Pyrrolopyridine electropherograms obtained with G1, C18 and uncoated capillary columns at $15 \mathrm{KV}$ in a pH 4.0 phosphate buffer. ..... 172

Chapter 6 Discussion and Conclusion

Figure 6-1. G1 alcohol molecular structure............................................................ 178

Figure 6-2. C18 alcohol molecular structure............................................................ 179

Figure 6-3. EOF dependence on $\mathrm{pH}$ and coating type: $\mathrm{G} 1$ carbamate coated, C18 carbamate coated, and uncoated silica capillary columns.

Figure 6-4. Generation 2 poly(aryl ether) dendrimer molecular structure................. 181

Figure 6-5. The separation of (1) toluene, (2) naphthalene, and (3) phenanthrene obtained at different temperatures with a G1 coated olumn.

Figure 6-6. The electropherograms demonstrate the structural selectivity provided by PAMAM.

Figure 6-7. Electropherograms showing the difference in selectivity between SDS-MECC and SBD-EKC.

Figure 6-8. The molecular structures of the three possible impurities 


\section{List of Symbols}

A cross-sectional area

cmc critical micelle concentration

$\mathrm{C}_{(\mathrm{K})}$ mass transfer coefficient in the mobile phase

$\mathrm{C}_{(\mathrm{K}) \mathrm{s}} \quad$ mass transfer coefficient in the stationary phase

d capillary diameter

$\mathrm{d}_{\mathrm{c}} \quad$ capillary inner diameter

$d_{f} \quad$ stationary phase thickness

dt change in time

$\mathrm{dH}$ rate of heat generation

D diffusion coefficient

$D_{m} \quad$ diffusion coefficient of solute in the mobile phase

$D_{s}$ diffusion coefficient of the solute in the stationary phase

E electric field strength

H theoretical plate height

i current

K distribution coefficient

L capillary length

$L_{d} \quad$ effective capillary length

$L_{1}$ total capillary length

N separation efficiency

pI isoelectric point

Q net charge of the particle

$\mathbf{r} \quad$ ionic radius

$R$ resistance

$\mathbf{R}_{\mathbf{s}} \quad$ resolution

t migration time

$t_{0} \quad$ migration time of the neutral marker

V applied voltage

$\alpha$

separation factor 


$\begin{array}{ll}\delta & \text { thickness of electrical double layer } \\ \varepsilon & \text { dielectric constant } \\ \zeta & \text { zeta potential } \\ \eta & \text { viscosity } \\ \mathbf{K} & \text { solution conductivity } \\ \mathrm{K} * & \text { retention factor } \\ \mu & \text { micron } \\ \mu_{\mathrm{m}} & \text { micrometer } \\ \mu_{\text {app }} & \text { apparent solute mobility } \\ \mu_{\text {avg }} & \text { average electrophoretic mobility of two solutes } \\ \mu_{\text {eof }} & \text { coefficient of the electroosmotic mobility } \\ \mu_{\text {ep }} & \text { electrophoretic mobility } \\ \mu_{+} & \text {cation mobility } \\ \mu_{-} & \text {anion mobiltiy } \\ \mu_{0} & \quad \text { neutral molecule mobility } \\ \nu & \text { average zone velocity } \\ \Delta v & \text { difference in zone velocity } \\ \sigma^{2} & \text { migrating zone width }\end{array}$




\begin{tabular}{|c|c|}
\hline \multicolumn{2}{|c|}{ Abbreviations } \\
\hline al & aliphatic \\
\hline ar & aromatic \\
\hline as & asymmetric \\
\hline $\mathbf{A r}_{0}$ & generation 0 aromatic ring \\
\hline $\mathrm{Ar}_{1}$ & generation 1 aromatic ring \\
\hline $\mathrm{Ar}_{2}$ & generation 2 aromatic ring \\
\hline AFM & atomic force microscopy \\
\hline $\mathrm{CE}$ & capillary electrophoresis \\
\hline CEC & capillary electrokinetic chromatography \\
\hline CIEF & capillary isoelectric focusing \\
\hline CITP & capillary isotachophoresis \\
\hline CGE & capillary gel electrophoresis \\
\hline CTAB & cetyltrimethylammonium bromide \\
\hline $\mathrm{CZE}$ & capillary zone electrophoresis \\
\hline $\mathrm{C}_{8}$ & eight carbon chain \\
\hline $\mathrm{C}_{18}$ & eighteen carbon chain \\
\hline d & doublet \\
\hline dd & distilled deionized \\
\hline EOF & electroosmotic flow \\
\hline ESI & electrospray ionization \\
\hline FTIR & fourier transform infrared \\
\hline $\mathrm{G}_{0}$ & generation 0 \\
\hline $\mathbf{G}_{1}$ & generation 1 \\
\hline $\mathbf{G}_{2}$ & generation 2 \\
\hline HEPES & N-2-hydroxyethylpiperazine-N'-2-ethanesulfonic acic \\
\hline${ }^{1} \mathrm{H}-\mathrm{NMR}$ & proton nuclear magnetic resonance \\
\hline HPLC & high performance liquid chromatography \\
\hline i.d. & internal diameter \\
\hline LC & liquid chromatography \\
\hline LIF & laser induced fluorescence \\
\hline OTC & open tubular capillary \\
\hline OT-CEC & open tubular capillary electrochromatography \\
\hline pI & isoelectric point \\
\hline Ppm & parts per million \\
\hline Pyx & pyridine \\
\hline
\end{tabular}




\begin{tabular}{|c|c|}
\hline$q$ & quartet \\
\hline $\mathbf{R}$ & resolution \\
\hline $\mathrm{R}_{\mathbf{f}}$ & migration factor \\
\hline $\mathbf{R P}$ & reversed phase \\
\hline $\mathbf{s}$ & singlet \\
\hline st & stretching \\
\hline sy & symmetric \\
\hline SDBS & sodium dodecyl benzene sulfonate \\
\hline SDS & sodium dodecyl sulfonate \\
\hline $\mathbf{t}$ & triplet \\
\hline$t_{r}$ & retention time \\
\hline$t^{3}$ & relative retention time \\
\hline TEOS & triethoxysilane \\
\hline TLC & thin layer chromatography \\
\hline TMS & tetramethylsilane \\
\hline TRIS & tris(hydoxymethyl-aminomethane) \\
\hline UV & ultraviolet \\
\hline $\mathbf{w}$ & peak width \\
\hline
\end{tabular}




\section{CHAPTER 1}

\section{Introduction}

\subsection{Introduction}

Capillary electrochromatography (CEC) is a technique that combines the desirable features of both high performance liquid chromatography (HPLC) and capillary electrophoresis (CE). It can be performed in open tubular capillary columns (OTC) $)^{1-8}$ and in packed capillary columns (PCC). 9,10 Unlike HPLC, where the bulk flow is driven by hydrostatic pressure which gives a laminar flow profile, CEC employs a solvent flow generated by electroosmosis to give a flat profile. The separation process is based on differential interactions between the stationary and mobile phases, while the electroosmotic flow (EOF) transports the mobile phase through the capillary. This technique differs from a traditional $\mathrm{CE}$ because $\mathrm{CE}$ cannot separate uncharged species due to the lack of a retention mechanism. For uncharged species, separation in CEC is achieved by interaction of solutes with the stationary phase. The separation of charged analytes is based on both electrophoretic mobility and partitioning between the stationary phase and the mobile phase. The stationary phase can be particles fixed in a packed bed as in HPLC, or a thin film on the inner wall of capillary.

Capillary electrophoresis (CE) has been widely exploited as a powerful separation tool for the analysis of a large variety of complicated samples. However, unmodified fused silica capillaries have two inherent problems: (1) the electroosmotic flow is not reproducible, and (2) many charged molecules (e.g., basic proteins and basic compounds) adhere to the wall of the column. Permanent silanol modification appears to be the most 
elegant and practical method of controlling both electroosmotic flow and protein-wall interactions. Therefore, the search for new stationary phases supporting the CEC separation technique becomes an exciting challenge.

Extensive work has been published on wall modifications using commercially available linear polymers, such as polyethylene glycol, ${ }^{11-16}$ polyethyleneimine, ${ }^{13}$ nonionic surfactants, ${ }^{14}$ poly(vinylpyrrolidinone) ${ }^{15}$ and polymethacrylate. ${ }^{16}$ Recently, Guo and Colón 4,5 explored sol-gel technology to produce porous silica glass films that can be cast onto the inner walls of OTCs for LC and CEC applications.

Dendritic polymers are a relatively new class of polymers with structures that depart rather dramatically from traditional linear polymers. These molecules have gained considerable attention in the past 20 years due to their highly branched, monodisperse structures which can lead to a number of interesting characteristics and features. One of the most interesting features is the solution structure of large dendrimers, which may adopt a spherical shape like a micelle. This unusual physical property is exhibited by the variation in their intrinsic viscosities 17,18 with molecular weight. When the size increases beyond a certain point, the intrinsic viscosity begins to decline, contrary to the behavior of linear polymers. Figure 1-1 shows the intrinsic viscosity behavior of polyether dendrimers and polystyrene. This effect is believed to be a consequence of the globular shapes of high generation dendrimers leaving them unable to "tangle" with one another, as is characteristic of linear polymers. Figure 1-2 depict the structural and topological relationships between high generation dendrimers and of linear polymers. 19,20 


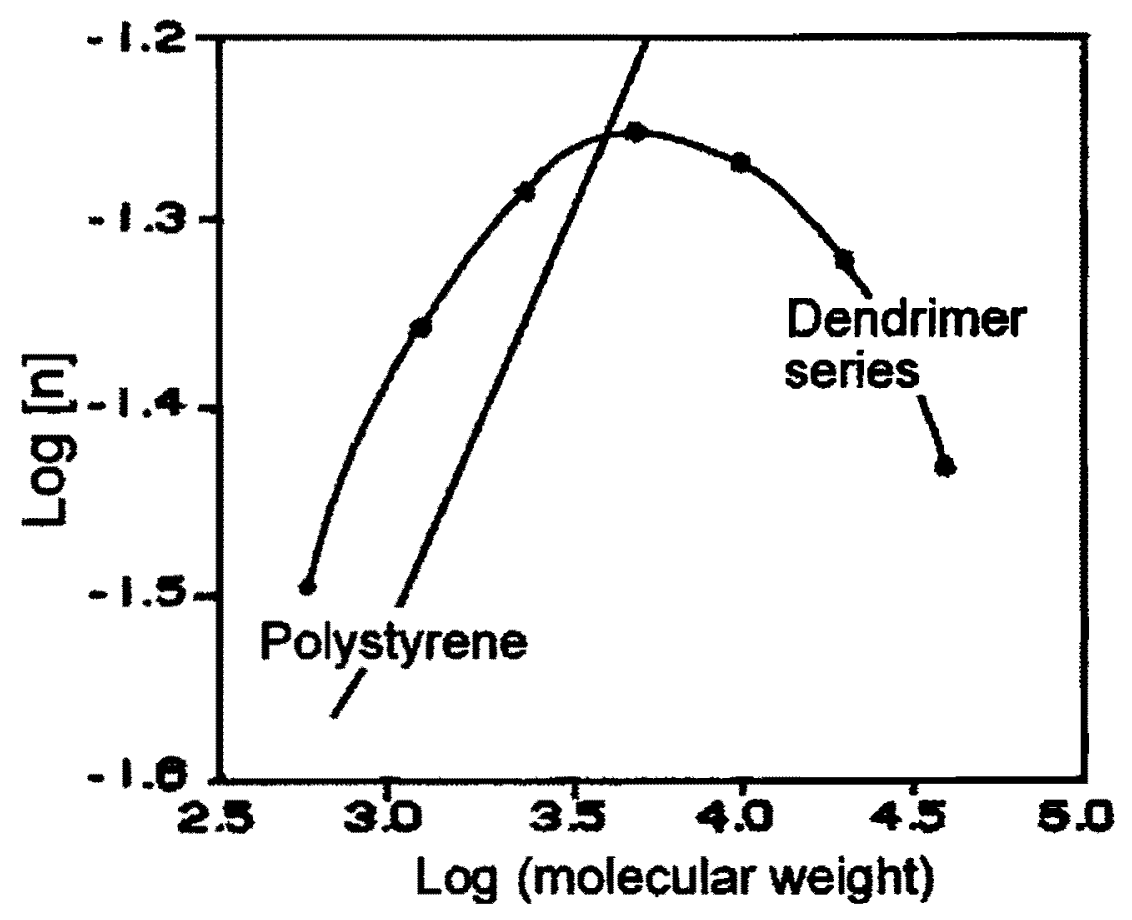

Figure 1-1. Intrinsic viscosity behavior of polyether dendrimers and polystyrene. 18

A large variety of dendrimers have been synthesized. Prominent examples are the polyamidoamines (PAMAM) of Tomalia, polyethers (Fréchet), arborols and metal containing structures (Newkome) and polyamines (Meijer). Researchers have shown promising results using polyamidoamine starburst dendrimers (PAMAM-SBDs) as pseudo-stationary phases in micellar electrokinetic capillary chromatography (MECC). $21-24$ 


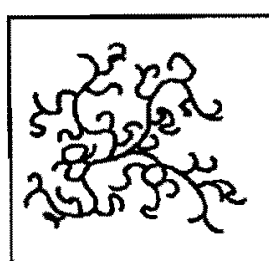

(a)

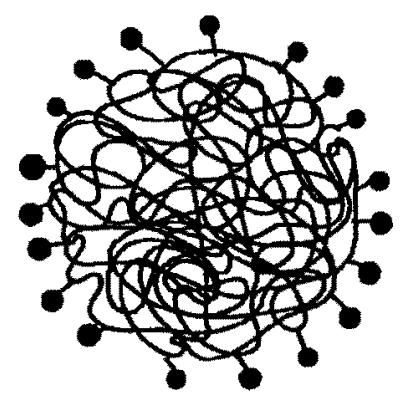

(d)

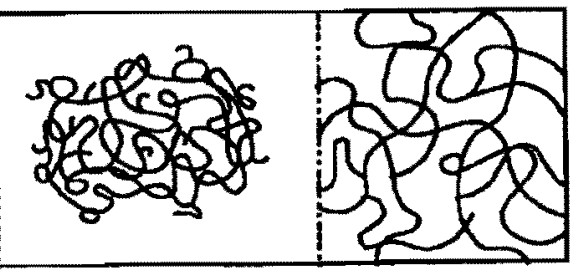

(b)

Figure 1-2. Structural and topological relationships between linear polymers shown in (a) branched polymers; (b) $\mu$ - gels; (c) macroscopic networks; and (d) functionalized $\mu$-gels; and the dendrimers shown in (e) cascade polymers. 19

\subsection{Scope of the research}

This study has focused on the investigation of OT-CEC in which a stationary phase is bonded inside a capillary tube by a covalent bond. We have synthesized and designed a reaction scheme for linking neutral dendrimers to the silanol wall. In this study, the first generation (G1) and second generation (G2) of poly(aryl ether) alcohols were synthesized. Figure 1-3 provides an example for the poly(aryl ether) dendrimer structures developed by Fréchet with the generations clearly defined. 


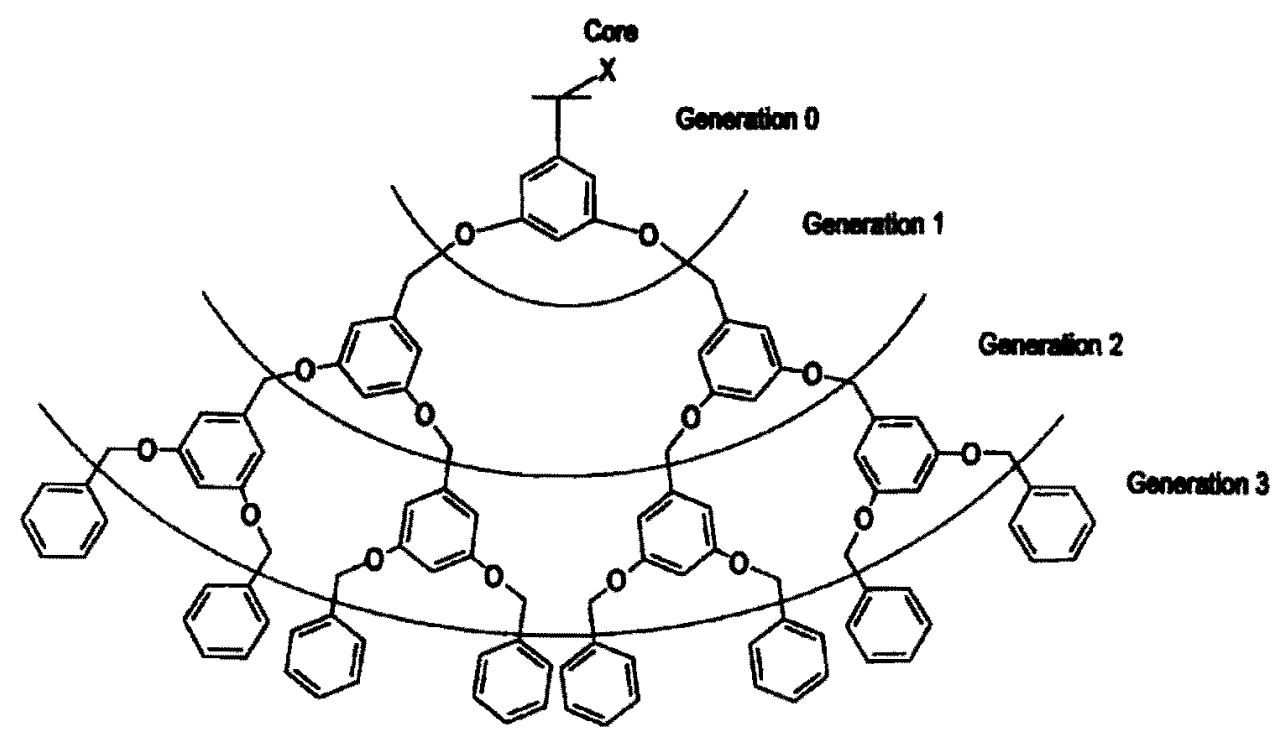

Figure 1-3. An example of a small poly(aryl ether) monodendron based on the work of Fréchet. The terminology of "generations" is roughly indicated by the circular zones. This is a third generation monodendron: there are three layers of the polymer added to the core (where $\mathrm{X}=\mathrm{OH}$ or $\mathrm{Br}$ ). Note that each monomer branches into two chains (a branching ratio of two) and that the end groups are the highest generation. 25

A new method to chemically bond the dendritic polymers onto the inner wall of a fused silica capillary column was developed using tri-(ethoxysilyl)propyl isocyanate as an anchoring agent. Five different substrates were studied as bonded stationary phases in OT-CEC: (1) G0 alcohol (benzyl alcohol), (2) G1 alcohol, (3) G2 alcohol, (4) Stearyl 
alcohol (C18 straight chain), and (5) 4-pyridyl carbinol. Their chemical structures are shown in Figure 1-4. Model separations of different analytes were examined at various $\mathrm{pH}$ values and in a variety of buffer electrolytes.

It is our hypothesis that the permanent silanol surface modification with dendrimers as stationary phases would provide better control of electroosmotic flow and prevent the undesirable analyte-wall interaction due to electrostatic interaction.

\subsection{Outline of the dissertation}

The focus of this dissertation is to investigate the potential of poly(aryl ether) dendritic polymers as bonded stationary phases. To facilitate discussion of the research presented later in this dissertation, Chapter 2 reviews basic theories and general practices of the capillary separation techniques under investigation. A general description of the dendritic polymer technology and the rationale for using this material as stationary phases in open tubular capillary electrochromatography are presented in Chapter 3. The synthesis of the dendritic polymers, the coating materials and the spectroscopic structural identification and characterization of the synthesized materials are provided in Chapter 4. Chapter 5 describes the details of the capillary column preparation protocols and performance evaluation for the surface modified capillaries. Chapter 6 includes discussion, conclusion and future prospects. The references are listed at the end of each chapter. 
<smiles>CCOCCCCNC(=O)OCc1cc(OCc2ccccc2)cc(OCc2ccccc2)c1</smiles><smiles></smiles><smiles>CCCCCCCCCCCCCCCCCCCOC(=O)NCCC[SiH2]OCC</smiles>

(11) C18 carbamate<smiles>CCOCCCCNC(=O)OCc1ccncc1</smiles>

(12) Pyridyl carbinol carbamate<smiles>CCOCCCCNC(=O)OCc1ccccc1</smiles>

(13) Benzyl carbamate

Figure 1-4. Chemical structure of the five coating materials. 


\subsection{References for Chapter 1}

1) Bruin, G. J. M.; Tock, P. P. H.; Kraak, J. C.; Poppe, H. J. Chromatogr. 1990, 517, 557-572.

2) Chiari, M.; Dell'Orto, N.; Gelain, A. Anal. Chem. 1996, 68, 2731-2736.

3) Fu, R.; Liu, Y.; Gu, J. J. Chromatogr. A 1995, 694, 498-506.

4) Guo, Y.; Colón, L. A. J. Microcol. Sep. 1995, 7, 485.

5) Guo, Y.; Colón, L. A. Anal. chem. 1995, 67, 2511.

6) Lagu, A.; Strege, M. Anal. Chem. 1991, 63, 1233-1236.

7) Lee, M.; Huang, M. J. Microcol. Sep. 1992, 4, 491-496.

8) Schurig, V.; Mayer, S. J. of Liq. Chroma. 1993, 16, 915-931.

9) Jorgenson, J. W.; MacNaiar, J. E.; Lewis, K. C. Anal. Chem. 1997, 69, 983-989.

10) Yan, C.; Dadoo, R.; Zhao, H.; Zare, R. N.; Rakestraw, D. J. Anal. Chem. 1995, $67,2026$.

11) Bruin, G.; Chang, J.; Kuhlman, K.; Zegers, K,; Kraak, J.; Poppe, H. J. Chromatogr., 1989, 471, 429.

12) Herren, B. J.; Shafer, S. G.; Alstine, S. V.; Harris, J. M.; Snyder, R. S. J. Colloid Interface. Sci. 1987, 115, 46.

13) Towns, J. K.; Regnier, F. E. J. Chromatogr., 1990, 516, 1126.

14) Towns, J. K.; Regnier, F. E. Anal. Chem. 1991, 63, 1126.

15) McCormick, R. M. Anal. Chem., 1988, 60, 2322.

16) Remcho, V. T.; Tan, Z. J. J. Microcolumn Separations, 1998, 10, 99-105.

17) Cai, C.; Chen, Z. Y. Macromolecules 1998, 31, 6393-6396. 
18) Fréchet, J. M. J.; Hawker, C. J.; Wooley, K. L. J. Macromol. Sci., Pure Appl. Chem. 1994, A31, 1627.

19) Obrecht, W.; Seitz, U.; Funke, W. Macromol. Chem. 1978, 179, 2145.

20) Antonietti, M. Angew. Chem., Int. Ed. Engl. 1988, 27, 1743.

21) Terabe, S.; Tanaka, N.; Tanigawa, T.; Hosoya, K.; Kimata, K.; Araki, T. Chem. Lett. 1992, 959-962.

22) Tanaka, N.; Fukutome, T.; Hosoya, K.; Kimata, K. a. A., T. Journal of Chromatog. A 1995, 716, 57-67.

23) Meijer, E. W.; Jansen, J. F. G. A.; Muijselaar, P. G. H. M.; Claessens, H. A.; Cramers, C. A. J. High Resol. chromatogr. 1995, 18, 121.

24) Castagnola, M.; Cassiano, L.; Lupi, A.; Messana, I.; Patamia, M.; Rabino, R.; Rossetti, D. V.; Giardina, B. J. of Chromatogr. 1995, 694, 463-469.

25) Hanlon, P. M., Ph.D. Dissertation, Seton Hall University, 1998. 


\section{CHAPTER 2}

\section{Capillary Electrochromatograpphy}

\section{$2.1 \quad$ Introduction}

Capillary electrophoresis (CE) and open tubular capillary electrochromatography (OT-CEC) are the separation techniques used in this research study. CEC, in general, is considered to be one of the operational modes of $\mathrm{CE}$. The principle involved in $\mathrm{CE}$ separation is based purely on the electrophoretic mobility of each analyte, but the OTCEC technique combines electrophoresis and reversed-phase liquid chromatography to separate both charged and uncharged species. This chapter will describe operational theories and general practice of both CE and CEC techniques.

\subsection{Capillary electrophoresis (CE)}

Capillary electrophoresis (CE) has shown a tremendous growth during the past decade. This technique offers high speed, high efficiency, great mass sensitivity, low consumption of analytes, and high resolution. The concept of electrophoresis dates back more than a century ago as documented by Compton and Brownlee. 1 The first report on electrophoresis in a tubular format appeared in the late $19^{\text {th }}$ century, where Hardy observed globulin movement within a U-tube under the influence of an electric field. ${ }^{2}$ Modern capillary electrophoresis was born in 1964, when Martin and Everaerts described the technique of displacement electrophoresis that was presented in Everaerts' doctoral thesis. 3 Their publication laid the foundation for performing electrophoretic separations in capillaries and also described methods for in-line detection of the separated zones. 
In 1967, Hjerten $^{4}$ provided the earliest demonstration of the use of high electric field strength in free solution electrophoresis in $3 \mathrm{~mm}$ i.d. capillaries. Pioneering work done by Verheggen, Mikkers, and Everaerts further illustrated the advantages of using small i.d. capillaries. However, they were unable to demonstrate high separation efficiencies because of sample overloading, poor detector sensitivity, and large injection volumes. 5,6 In 1981, CE became an attractive technique when Jorgenson and Lukacs solved the perplexing problems of injection and detection using $75 \mu \mathrm{m}$ i.d. capillaries and established the fundamental theory of CE. 7-10

The basic principles of CE are simple. A schematic diagram of a prototypical CE instrument illustrating the experimental setup is shown in Figure 2-1. This CE instrument consists of a high-voltage power supply, two buffer reservoirs, two electrodes, a detector and a data acquisition system. In the CE operation, both ends of the capillary are placed in the reservoirs containing the buffer electrolyte. A small plug of sample solution is introduced into one end of a fused silica capillary tube. Component molecules in the injected sample then migrate at different rates along the length of the capillary separation channel under the action of a large electrical field, and the molecules are detected as they approach the other end of the capillary by one of several on-line detection schemes. Online detection is most often performed either by using an UV spectrophotometric detector or a laser induced fluorometry (LIF) detector. The end-column approach is used when other detection devices are interfaced with CE (i.e., a mass spectrometer or an electrochemical detector). 11,12 
The key to this efficient separation technique is the inherently large internal surface-area-to volume ratio. Small i.d. capillaries $(<100 \mu \mathrm{m})$ allow efficient heat dissipation. This heat removal limits the broadening of sample zones, and higher theoretical plate counts can therefore be achieved.

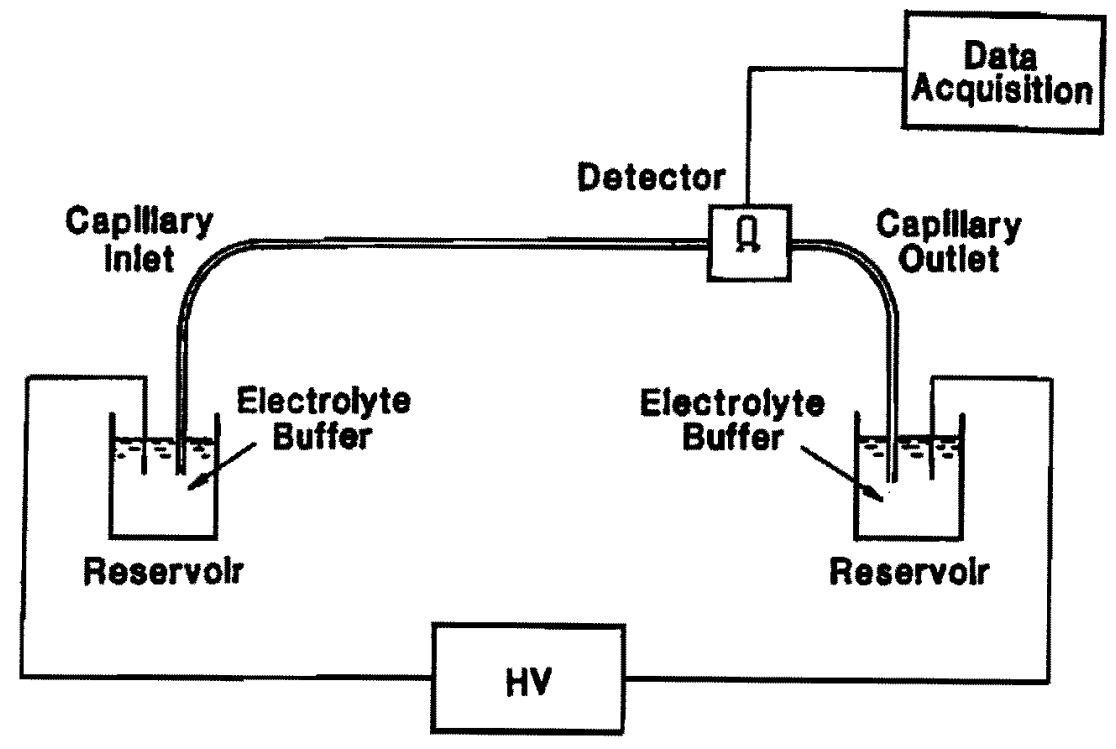

Figure 2-1. Diagram of a basic capillary electrophoresis system. 


\subsubsection{Modes of capillary electrophoresis.}

While the high efficiencies of capillary electrophoretic separations are achieved through the use of sufficiently small diameter separation channels, selectivity for sample components of interest is obtained by the choice of the proper separation medium within the capillary. To achieve highly efficient separation, many modes of CE separation have been developed. They are;

1. Capillary zone electrophoresis (CZE)

2. Capillary gel electrophoresis (CGE)

3. Capillary isoelectric focusing (CIEF)

4. Capillary isotachophoresis (CITP)

5. Micellar electrokinetic capillary chromatography (MECC)

6. Capillary electrochromatography (CEC)

CZE, which is now commonly referred to as CE (also in this thesis), is the most mature of the six modes of operation. CE separations are based upon differences in the electrophoretic mobilities of charged species, resulting in different migration velocities under the influence of the applied electric field. ${ }^{13}$ The separation mechanism, which will be discussed in this chapter, is related to solute size and charge at a given $\mathrm{pH}$ value. High resolution separations based on size differences of biopolymers is referred to as CGE mode. 14,15 The technique is directly comparable to traditional slab gel electrophoresis since the separation mechanisms are identical. Polyacrylamide gel-filled capillaries are usually employed as the separation media. However, the technique is limited to neutral molecules since electroosmotic flow is suppressed in this mode of operation. CIEF ${ }^{13,16-}$ 
18 is a high resolution electrophoretic technique used primarily to separate proteins and peptides on the basis of $\mathrm{pI}$. In this technique, a $\mathrm{pH}$ gradient is formed within the capillary using ampholytes. After filling the capillary with sample and ampholytes, the gradient is formed. With a basic solution at the cathode and an acidic solution at the anode, and upon application of the electric field, the charged ampholytes and proteins migrate through the medium until they reach a region where they become uncharged (at their $\mathrm{pl}$ ). This process is known as "focusing". After focusing, the solutes and ampholytes are mobilized and the zones passed through the detector. EOF needs to be reduced or eliminated in CIEF since the flow could flush the ampholytes from the capillary before focusing is complete. Reduction of EOF can be accomplished by the use of dynamic or covalent coatings. The coating is also helpful in limiting protein adsorption to the capillary walls. CITP13,19 differs from other modes of CE. It is performed in a discontinuous buffer system. In a CITP separation, charged analytes migrate between two solutions of different ionic mobility (i.e. leading electrolyte and terminating electrolyte). Separation will occur in the gap between the leading and terminating electrolytes based on the individual mobilities of the analytes. Both anions and cations can be determined, although not in the same run. Perhaps the most intriguing mode of CE for the determination of small molecules is MECC. 13,20,21 In MECC, micelles are used in the electrolyte buffer at a concentration above their critical micelle concentrations (cmc). They act as a pseudo-stationary phase in solution that resembles reverse-phase LC with the benefits of CE. Neutral molecules are separated based on differential partitioning between the micelle phase and electrolyte phase. Finally, the CEC mode offers the true bridge between capillary HPLC and CE. 
Since this study is focused on open tubular capillary electrochromatography, its fundamental theory and separation mechanism will be discussed in detail in section 2.3.

\subsubsection{Electroosmotic Flow.}

One of the most distinguishing properties of capillary electrophoresis is electroosmotic flow (EOF), which refers to the movement of solvent relative to a stationary charged surface due to an applied electric field. 22,23 In CE, electroomosis causes nearly all species, regardless of charge, to migrate in the same direction, and it plays a principal role in many of the modes of CE, such as CZE, MECC and CEC.

The EOF results from the effect of the applied electric field on the solution doublelayer at the wall. Under aqueous conditions most solid surfaces possess an excess of negative charges. This can result from ionization of the surface and/or from adsorption of ionic species at the surface. For fused silica, both processes probably occur. Silanol groups present on the inner walls of the fused silica capillary are ionizable at $\mathrm{pH} 2.0$ or higher. Therefore, the interior surface of the fused silica capillary is negatively charged under normal CE conditions. Positive ions are then attracted towards the negative surface and negative ions are repelled. A fraction of the ions forming the electrolyte part of the electric double layer is always fixed by electrostatic forces near the capillary wall and forms the so called Stern layer. Further away from the silica surface are the remaining excess ions, which are solvated and behave like any other ion in the adjacent solution in an applied electric field. This region is called the diffuse layer or Gouy-Chapman layer, as shown in Figure 2-2. 24-26 The potential at the boundary between the Stern layer and the diffuse layer is called the zeta potential $(\zeta)$, typically between 0 and $100 \mathrm{mV}$. The value 
of the zeta potential is dependent not only on the surface charge but also on the electrolyte concentration. At higher electrolyte concentrations, the potential drop is steeper, and consequently, the zeta potential is lower. The zeta potential drops exponentially with the distance (x) from the capillary wall, and is given by the Helmholtz equation. 4,27

$$
\zeta=\frac{4 \pi \eta \mu_{\mathrm{eof}}}{\epsilon}
$$

Where $\eta$ is the viscosity, $\epsilon$ is the dielectric constant of the solution, and $\mu_{\text {eor }}$ is the coefficient of the EOF or electroosmotic mobility. The distance $(\delta)$, over which the zeta potential decrease by the factor of $\mathrm{e}^{-1}$, is the thickness of the electrical double layer.

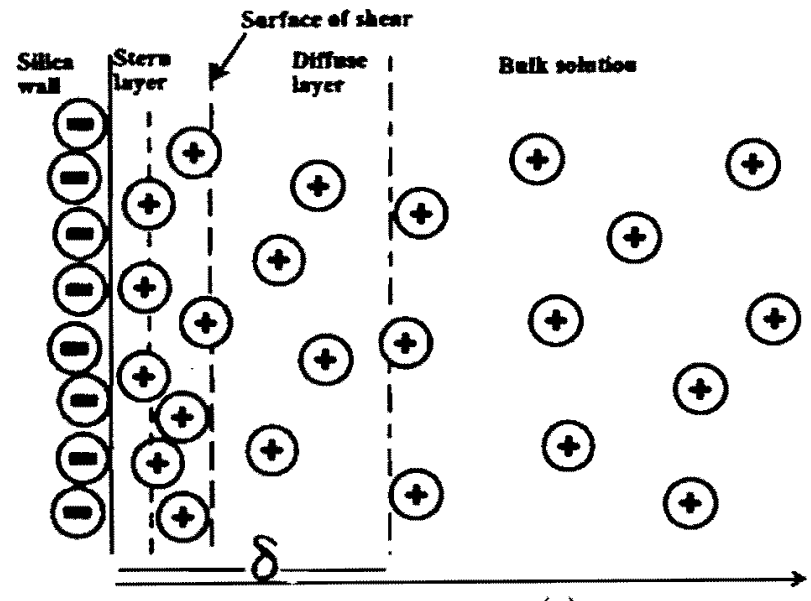

Detence frem captitury wall $(\mathrm{x})$

Figure 2-2. Representation of the double-layer at the capillary wall. 28

When voltage is applied across the fused silica capillary, the cations forming the diffuse double-layer are attracted toward the cathode, producing a pluglike flow that has a 
flat velocity distribution across the capillary diameter. $7,9,10$ This process is shown in schematic form in Figure 2-3.

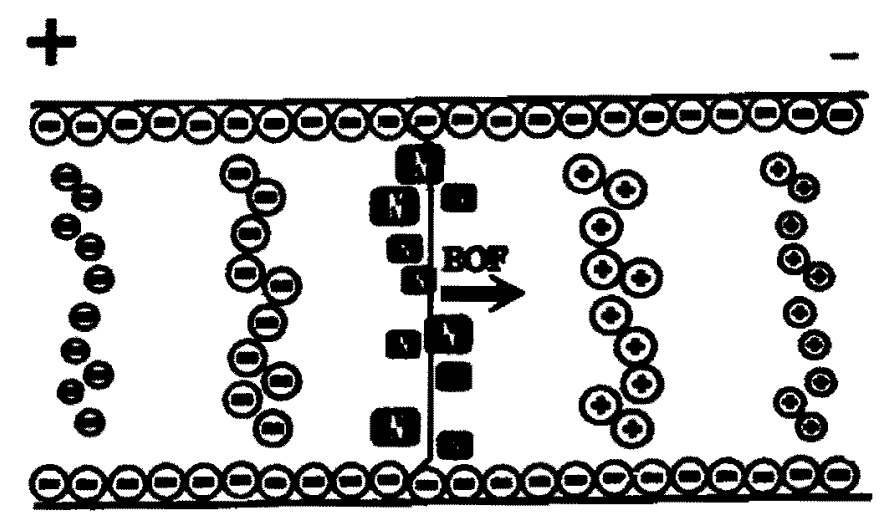

Figure 2-3. Differential solute migration superimposed on electroosmotic flow in capillary zone electrophoresis.

Experimentally, the EOF is measured by monitoring the migration time of an uncharged EOF marker. The electroosmotic mobility, $\mu_{\text {oof }}$ can be calculated by the following equation. 13

$$
\mu_{\text {eof }}=\frac{v_{\text {eof }}}{E}
$$

where $v_{\text {eor }}$ is the velocity of the EOF, and $\mathbf{E}$ is the field strength. Using an experimentally measurable parameter, Eq. 2.2 can be rewritten:

$$
\mu_{\text {eof }}=\frac{L_{d} L_{t}}{t_{0} V}
$$


where $\boldsymbol{L}_{d}$ from the point of injection to the point of detection, $\boldsymbol{L}_{t}$ is the total length of the capillary, $t_{0}$ is the migration time of the EOF marker, and $V$ is the applied voltage.

The EOF can be influenced by $\mathrm{pH}$, ionic strength of the background electrolyte, and organic solvent additives. ${ }^{13,19}$ At high $\mathrm{pH}$, the silanol groups on the inner surface of the fused silica capillary are fully ionized, thus generating a large zeta potential and a dense double layer. As a result, the EOF increases as the buffer $\mathrm{pH}$ is elevated. Figure 2-4 illustrates this effect for fused silica and other materials. ${ }^{29}$

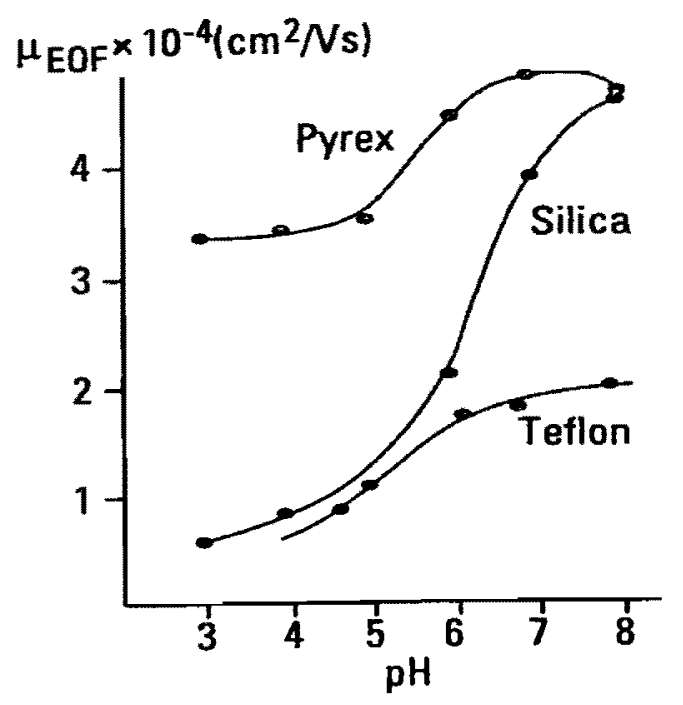

Figure 2-4. Effect of $\mathrm{pH}$ on electroosmotic flow mobility in various capillary materials. 29

EOF can also be affected by adjusting the concentration and ionic strength of the buffer electrolyte. High buffer concentrations decrease the effective charge at the inner wall, while increased ionic strength results in double layer compression, decreased zeta potential, and reduced EOF (Figure 2-5). The addition of simple organic solvents can 
influence EOF dramatically. ${ }^{30}$ Methanol decreases electroosmotic flow while acetonitrile increases electroosmotic flow, although not as dramatically. The addition of organic solvents generally decreases the EOF. ${ }^{30-32}$ The EOF can also be controlled by modification of the capillary wall by means of dynamic coatings or covalent coatings. These coatings can increase, decrease, or reverse the surface charge and thus control the EOF. 33,34
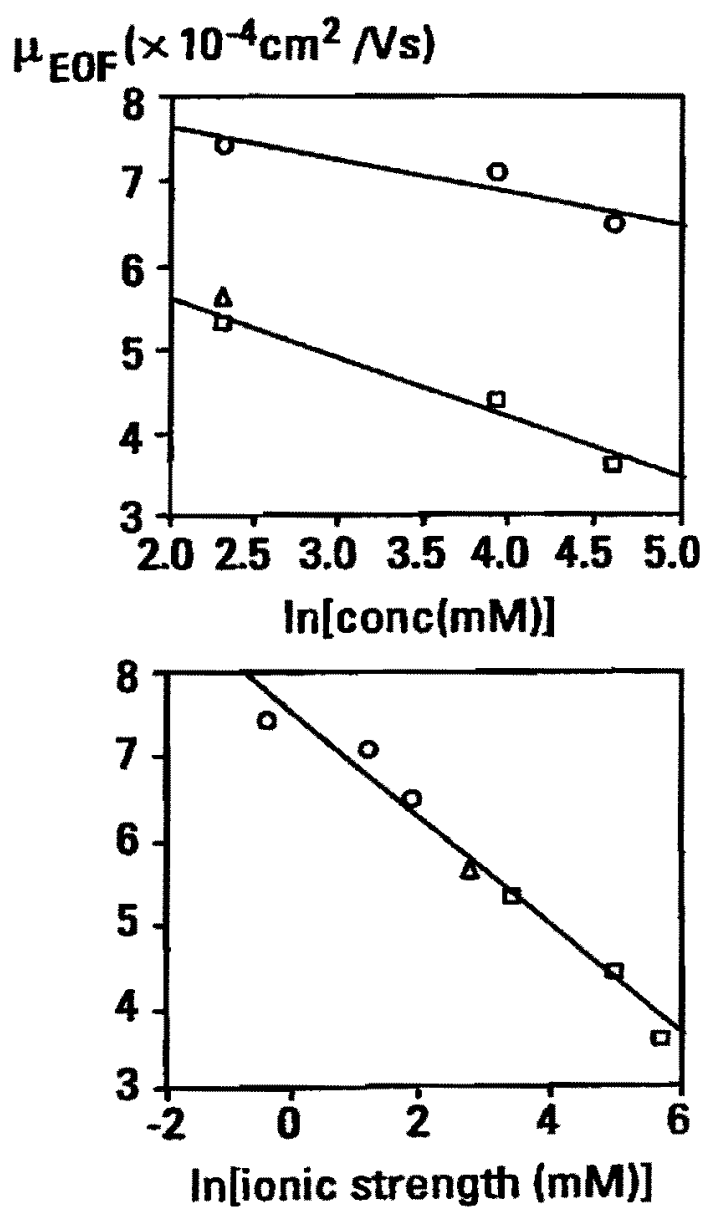

Figure 2-5. Electroosmotic flow mobility as a function of buffer concentration and ionic strength. 35 


\subsubsection{Theory of CZE operation}

In this section, the principles of electrophoretic migration in capillaries relative to migration time and efficiency, and the physical phenomena that affect the nature of separation are discussed. The discussion in this section primarily concerns aspects of capillary zone electrophoresis in fused silica capillaries. However, the same principles can be applied to other capillary electrophoretic techniques.

Migration velocity. As described in the beginning of this chapter, electrophoresis is the separation of charged molecules based on differential migration in an applied potential field. The ionic species migrate in the buffer solution with an electrophoretic mobility (direction and velocity) determined by their charge and mass. Electrophoretic mobility $\left(\mu_{\mathrm{cp}}\right)$ can be approximated from the Debye-Huckel-Henry theory, 19

$$
\mu_{\text {ep }}=\frac{\mathrm{Q}}{6 \pi \eta r}
$$

where $\mathbf{Q}$ is the net charge on the particle, $\boldsymbol{\eta}$ is the viscosity of the buffer, and $\boldsymbol{r}$ is the ionic radius. The size of the molecule, usually characterized by its ionic radius or Debye radius, is related to its molecular weight, three-dimensional structure, and degree of solvation. With CZE, the normal polarity is considered to be from inlet $[+]$ to outlet $[-]$ as shown in Figure 2-1. Without considering electroosmosis, the migration velocity 0 in electrophoresis can be expressed by the following equation,

$$
v_{\text {ep }}=\mu_{\text {ep }} \mathrm{E}=\frac{\mu_{\text {ep }} V}{L}
$$


where $\mathrm{E}$ is the potential field strength $(V / L), V$ is the voltage applied across the capillary, and $L$ is the capillary length. The migration time $t$ for a solute electrophoretically migrating from one end of the capillary to the other is given by

$$
t=\frac{L}{u_{\text {ep }}}=\frac{L^{2}}{\mu_{\text {ep }} V}
$$

If the applied field were the only force acting on the ions, net positively charged (cationic) substances would pass the detector while neutral components would remain static (i.e. at the inlet) and anionic components would be driven away from the detector. It is clear that if this were the case, $\mathrm{CE}$ would be of limited use. Fortunately, the migration of all species in fused silica capillaries is not solely governed by their electrophoretic mobilities. The bulk flow of the electrolyte solution (i.e. the EOF) has a direct effect on the net migration velocity of charged species, and in this sense both the separation efficiency and resolution are related to the flow rate. Thus, in the presence of electroosmotic flow, the migration velocity and time from Eqs. 2.5 and 2.6 can be rewritten as

$$
v_{\text {ep }}=\frac{\left(\mu_{\text {ep }}+\mu_{\text {oof }}\right) V}{L}
$$

and

$$
t=\frac{L^{2}}{\left(\mu_{\text {ep }}+\mu_{\text {off }}\right) V}
$$

In Eqs 2.7 and 2.8, the solute velocity and the migration time are now proportional to the sum of the coefficients for electrophoretic mobility and electroosmotic flow. $7,9,10$ If the rate of EOF is greater in magnitude and opposite in direction to all anions in the buffer, 
then all ions will migrate in the same direction, and the nonionic species will be carried by the EOF and elute at one end of the capillary. Figure 6 shows the effect of electrophoretic migration and electroosmotic flow on the elution order of cations, neutrals, and anions in CE. The electroosmotic or electrophoretic mobility in the cathodic direction normally takes the positive sign. It can be noted from Eq. 2.8 that higher voltages and shorter column lengths provide faster elution times in $\mathrm{CE}$.

Separation efficiency. The charged molecules in a sample matrix are separated into discrete zones migrating at the velocity determined by Eq. 2.7. If there is no solutewall interaction, the only contribution to band broadening is longitudinal diffusion, therefore the variance of the migrating zone width $\left(\sigma^{2}\right)$ is determined by Einstein's law of diffusion, which can be written in term of total migration time and is given by

$$
\sigma^{2}=2 \mathrm{D} t
$$

Substituting Eq. 2.8 into Eq. 2.9 yields:

$$
\sigma^{2}=\frac{2 \mathrm{D} L^{2}}{\left(\mu_{\mathrm{ep}}+\mu_{\mathrm{eof}}\right) V}
$$

where $D$ is the diffusion coefficient of the solute. The separation efficiency $(N)$ expressed by the number of theoretical plates using the statistical equivalence of variance is given by:

$$
N=\frac{L^{2}}{\sigma^{2}}=\frac{\left(\mu_{\mathrm{ep}}+\mu_{\text {eof }}\right) V}{2 \mathrm{D}}
$$

From Eq. 2.11, it is clear that the separation efficiency is based upon applied voltage and not capillary length. Given the limitations of efficient heat dissipation and maximum 
separation efficiency, short analysis times are obtained at high voltages with short columns.

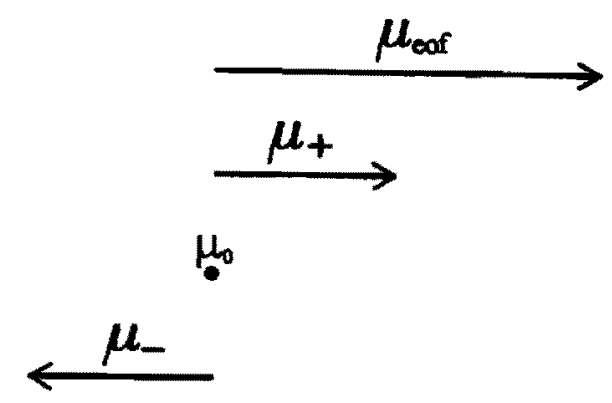

Net offect:

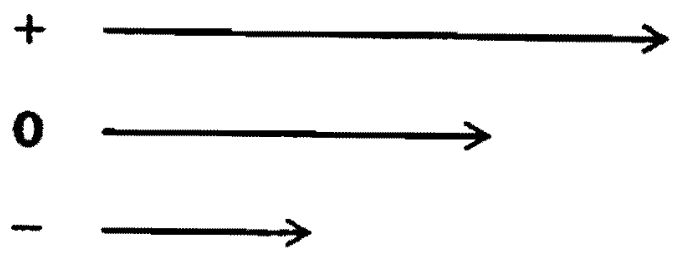

Figure 2-6. Elution order for cations ( + ), nonionics $(0)$, and anions $(-)$ based on the cumulative effects of electrophoresis and strong electroosmotic flow toward the cathode.

Resolution. In zone electrophoresis, ionic species are resolved on the basis of differential electrophoretic migration A general expression for resolution $(R s)$ of two zones in electrophoresis was derived by Giddings in 1969. 


$$
R s=\frac{1}{4} N^{1 / 2}-\frac{\Delta v}{v_{\text {avg }}}
$$

where $\Delta v$ is the difference in zone velocities, and $u_{\mathrm{mg}}$ is the average zone velocity. Substituting Eqs. 2.7 and 2.11 into Eq. 2.12, an equation for the resolution of two adjacent ions can be written as

$$
R s=\frac{1}{4(2)^{1 / 2}} \Delta \mu_{\text {ep }}\left[\frac{V}{D\left(\mu_{\text {avg }}+\mu_{\infty}\right)}\right]^{1 / 2}
$$

where $\Delta \mu_{\mathrm{mp}}$ is the difference in the electrophoretic mobilities and $\mu_{\mathrm{mg}}$ is the average electrophoretic mobility of the two solutes.

This expression suggests that increasing the voltage is a limited means of improving resolution. To double the resolution, the voltage must be quadrupled, which will cause a heating problem. According to Eq. 2. 13, the highest resolution is obtained when the net value of $\Delta \mu_{\mathrm{cp}}$ is increased. Control of $\Delta \mu_{\mathrm{ep}}$ is best accomplished through selection of the proper mode of capillary electrophoresis coupled with selection of the appropriate buffer electrolytes. Both of these areas will be covered later in Section 2.3 on capillary electrochromatography. From Eq. 2.13, it is also clear that the resolution of two zones will be poorer if there is a large component of electroosmotic flow in the same direction as electrophoretic migration. In fact, good resolution is obtained when $\mu_{\text {oof }}=-\mu_{\text {mong }}$. However, the cost of this approach to higher resolution is a longer analysis time. This is perceptible by referring to Eq. 2.8 and assuming that the electroosmotic flow coefficient and the electrophoretic mobility are equal in magnitude but opposite in direction. It is also noted that electroosmosis toward the cathode should result in better 
resolution of anions, which migrate against the electroosmotic flow and are carried back toward the cathode. Conversely, cations will be more poorly resolved under these conditions. Another way to increase the resolution is to increase the column length, longer columns with larger applied potential can be used to enhance resolution. However, the trade-off is a longer separation time.

The desired effect is to have the ability to control the rate of electroosmotic flow. There are several ways of manipulating the electroosmotic flow in capillaries. The most commonly used methods involve either changing the zeta potential across the solutionsolid interface or increasing the viscosity at the interface. One approach is to coat the capillary wall with a polymer such as methylcellulose or to covalently bond $\gamma$-methacryloxypropyl-trimethoxysilane to the fused silica surface. The electroosmotic flow rate can also be controlled by proper selection of the buffer electrolyte, buffer $\mathrm{pH}$, and buffer additives. It was observed that the direction of the bulk flow was reversed by adding cationic surfactants to the buffer electrolyte. 36

Joule heating. The production of heat is an inevitable result of the application of high field strengths. The capillary tube containing the electrophoretic medium behaves in a similar way as a cylindrical ohmic conductor when a voltage is applied across the two ends. Joule heating is a consequence of the resistance to the ionic current carried between the electrodes. Two major problems arise from heat production: temperature gradients across the capillary that increase zone broadening, and temperature changes due to ineffective heat dissipation that lead to changes in the migration velocities of the analytes 


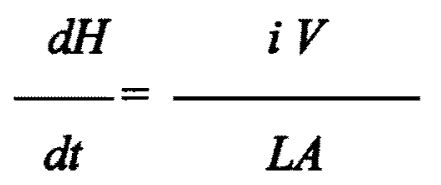

Eq. 2.14

Where $L$ is the capillary length, and $A$, the cross-sectional area. Since $i=V I R$ and $R=L / x A$, where $x$ is the conductivity of the solution, then Eq. 2.14 can be rewritten as: 29

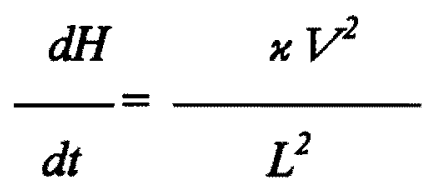

As noted in Eq. 2.15, the amount of heat generated is proportional to the square of the field strength. Either a smaller radius or a longer capillary results in a better ability to dissipate the heat generated at constant applied potential and buffer composition.

Heat loss from a horizontal tube in air is mainly by natural convection or by forced convection rather than by conduction through still air. A large amount of heat $\left(100 \sim 300 \mathrm{~W} / \mathrm{cm}^{2}\right)$ is released during a typical CE operation, due to the high applied potential. This can cause large temperature differences between the capillary walls and surrounding air under natural convection. Temperature excesses on the order of several degrees have been observed, and are responsible for the curvature of the EOF plot velocity vs applied voltage. 38 Both high-velocity air cooling $(10 \mathrm{~m} / \mathrm{s})$ and circulating liquid coolants have been applied to control the temperature rise. Theoretical modeling of the temperature effect on band broadening shows that the contribution of thermal convection to band broadening is negligible in small i.d. capillaries $(<75 \mu \mathrm{m})$. 


\subsection{Capillary electrochromatography (CEC)}

Capillary electrochromatography (CEC) is a relatively recent electrokinetic separation technique that can significantly improve chromatographic performance. The concept of employing an electric field as the driving force for the solvent flow in liquid chromatography was first introduce by Pretorius and coworkers in 1974. 39 Jorgenson, Lukacs, Knox, and Grant further demonstrated this technique's value, both theoretically and experimentally in the late 1980 s and early $1990 \mathrm{~s} .9,38,40$ Despite many practical problems in column technology, the technique is appealing to separation scientists and it has been applied to the analysis of a variety of substances, such as biological samples, pharmaceuticals, environmental pollutants, and pesticides. $41-44$

CEC may only be performed in a capillary column with a limited tube bore, less than $200 \mu \mathrm{m}$, due to self-heating. 37 The capillary columns can be either a packed capillary column (PCC) that was packed with HPLC stationary phase or an open tubular capillary (OTC) that was internally coated with a stationary phase. The separation mechanism for uncharged species is based on differential interactions between the stationary and mobile phases, while the electroosmotic flow (EOF) transports the mobile phase through the capillary. The separation of charged analytes is therefore based on both electrophoretic mobility and partitioning between the stationary phase and the mobile phase. The following discussion will focus on retention and efficiencies in both open tubular and packed column CEC. 


\subsubsection{Electroosmotic pumping}

CEC is considered a variant of HPLC where the eluent is driven by EOF. In PCC mode, fused silica capillaries are usually packed with silica particles $(1.5-5 \mu \mathrm{m}$ diameter) modified with stationary phases such as $\mathrm{C}_{8}$ and $\mathrm{C}_{18}$ alkyl chains; whereas in OTC the stationary phase is covalently bonded onto the inner walls of fused silica capillaries. In either case, silanol groups are still present on the surface of the particles or on the inner walls of the fused silica capillaries. As in the basic operational principle of CE, when a voltage is applied to a capillary, EOF is generated at the solid/liquid interface on the silica particles as well as on the inner walls of the capillary (The mechanism for EOF is explained in Section 2.2.2). The velocity profile of the EOF is considered to be flat. This contrasts with the parabolic flow profile observed in pressure-driven flows, as shown in Figure 2-7. This plug-like flat flow profile has the advantages of reducing band broadening and leading to high separation efficiencies in CEC. A decrease in EOF velocity was observed with packed capillaries $(\sim 40-60 \%$ slower $)$ and fused silica capillaries with modified surfaces when compared with unmodified capillaries. 40,45 In fact, the EOF is strongly influenced by the material used in the packed capillary and open tubular capillary column, 46,47 These observations suggested that not all material available for HPLC is suitable for CEC. In this research, we have explored the potential of using dendritic polymers, a relatively new polymer architecture, as the surface modifier in OT-CEC. 
(a)
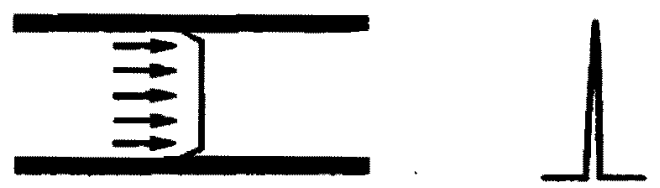

(b)
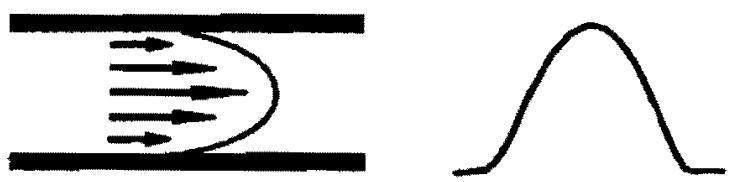

Figure 2-7. Flow profile in a capillary tube with (a) electro-drive, (b) pressure drive.

\subsubsection{Retention in CEC}

The essential difference between HPLC, CE, and CEC is the nature of the separation. CEC utilizes stationary phases prepared on packing particles or on the inner walls of fused silica capillaries to provide a retention mechanism. Hence two components are associated with the separative process in CEC. One involves solute retention based on differential partitioning between the mobile and the stationary phases (i.e, distribution ratio), similar to HPLC. The other component involves differential migration, characterized by the solute's electrophoretic velocity $0_{e p}$, as in CE, given by Eq. 2.7, Section 2.2.2, $v_{\mathrm{ep}}=\left(\mu_{\mathrm{pp}}+\mu_{\mathrm{cor}}\right) \mathrm{E}$.

Because of the coupled electrophoretic migration, the characteristic retention factor $x$, used in HPLC, is no longer valid for describing the migration process in CEC. In 
addition, the EOF velocity can significantly influence retention. 48 Fundamentally, $x$ is defined as the ratio of the separative to nonseparative components (i.e., the amount of analyte in the stationary phase and the amount of analyte in the mobile phase). An equivalent parameter in CEC must include both electrophoretic and chromatographic processes which are the characteristic separative components in CE and HPLC, respectively. An electrochromatographic retention factor $x^{*}$ that reflects the concurrence of chromatographic and electrophoretic process is given by: 49

$$
x^{*}=x+x\left(\mu_{\text {ep }} / \mu_{\text {eof }}\right)+\left(\mu_{\text {ep }} / \mu_{\text {oof }}\right)
$$

in which $x$ is the retention factor caused by chromatography alone in CEC. The electrophoretic component is expressed by the electrophoretic mobility normalized to the electroosmotic mobility (i.e. the electrophoretic velocity factor $\mu_{\mathrm{cp}} / \mu_{\mathrm{eof}}$ ). The second term on the right hand side of Eq. 2.16 expresses the product of the concurrence of the chromatographic and electrophoretic factors.

For neutral compounds, $\mu_{e p}=0$, so $x^{*}$ becomes $x$, reflecting a purely chromatographic process. However, for a charged, unretained compound (a purely electrophoretic process), $x=0$, and $x^{*}$ is given by the electrophoretic velocity factor. If the solute is charged and retained, in the stationary phase, then $x^{*}$ is given by the addition of the chromatographic and electrophoretic velocity factors and their convoluted concurrence.

The migration velocity of the sample component is related to $x$ through the sum of the electrophoretic and electroosmotic velocities multiplied by the retardation factor, giving 


$$
v_{s}=\frac{\left(\mu_{\text {ep }}+\mu_{\text {eof }}\right)}{(1+x)}
$$

Therefore, a more practical expression of $x^{*}$ is given by

$$
x^{*}=\left[\frac{\left(\mu_{\text {ep }}+\mu_{\text {eof }}\right)^{2}}{\left(\mu_{\text {eof }} \times v_{s}\right)}\right]-1 \quad \text { Eq. } 2.18
$$

The values for $\mu_{\text {oor }}$ and $b_{s}$ can be obtained experimentally from the electrochromatogram. 50

\subsubsection{Separation efficiency in CEC}

The first advantage of the EOF for CEC is the flat flow profile (see Figure 2-7). A flat profile precludes band broadening by trans-channel or radial diffusion. 51 This results in a significant increase in the number of theoretical plates, leading to high efficiencies in CEC. In practice, however, the EOF profile depends on both the capillary diameter $d$ and $\delta$ (see figure 2, section 2.22 .). In the theoretical study of Rice and Whitehead, 38 it was proposed that the profile would be pluglike for values of $d$ much greater than $\delta(d \geq 10 \delta)$. While there is no problem with double-layer overlap in CE in open tubes, for capillaries packed with the smallest particles possible it may represent a major problem. Based on this prediction, the author chose to focus this research study on CEC in the open tubular format.

A number of studies ${ }^{52-54}$ have been conducted using open tubular capillary electrochromatography (OT-CEC), and the effect of various surface modifications on the EOF has been investigated. Significant variations in EOF were observed for capillaries 
ranging from untreated to those etched and coated with the octadecylsilanyl group. 47 This variation in EOF velocity suggested that modification of the capillary wall may play an important role in fine tuning the separation processes. 24

This thesis is focused on OT-CEC application only. The separation efficiency involving the OT-CEC technique will be addressed in detail in the following section.

\subsubsection{Separation efficiency of OT-CEC}

According to HPLC theory, the theoretical plate height $(\boldsymbol{H})$ can be considered as the sum of different contributions to peak dispersion. 55 The terms that account for the theoretical plate height are: (1) axial diffusion of the solutes, (2) eddy diffusion, and (3) mass transfer constants in the mobile phase and stationary phase. Since the eddy diffusion is eliminated in OT-CEC, the Golay equation for plate height is given by

$$
H_{\mathrm{OT}-\mathrm{CEC}}=\left(\frac{2 \mathrm{D}_{\mathrm{m}}}{\mu}\right)+\left(\frac{\mathrm{C}(x)_{\mathrm{m}} d_{\mathrm{c}}^{2} \mu}{\mathrm{D}_{\mathrm{m}}}\right)+\left(\frac{\mathrm{C}(x)_{\mathrm{s}} d_{\mathrm{f}}^{2} \mu}{\mathrm{D}_{\mathrm{s}}}\right) \quad \text { Eq.2.19 }
$$

where $D_{m}$ and $D_{s}$ are the diffusion coefficients of the solute in the mobile and stationary phases, respectively; $\boldsymbol{\mu}$ is the linearity velocity; $\boldsymbol{d}_{\mathrm{c}}$ is the inner diameter of the capillary column and $d_{f}$ is the thickness of the stationary phase; and $C(x)_{m}$ and $C(x)_{t}$ are the masstransfer coefficients. In Eq. 2.19, the first term represents longitudinal diffusion, the second and the third terms represent mass transfer resistance in the mobile phase and stationary phase, respectively. The detailed mathematic treatment of Eq. 2.19 can be found in Giddings' book on Unified Separation Science. 56 
The mass-transfer coefficient in the mobile phase is affected by the flow velocity profile 57 and is expressed as

$$
C(x)_{m}=\frac{x^{2}}{16(1+x)^{2}}
$$

In practice, the separation efficiency also depends on the field strength for a given analysis time. Based on Eq. 2.7 and 2.17, for a neutral solute, the linear velocity and analysis time can be obtained using the following equations:

$$
v_{0}=\frac{\mu_{\text {off }} V}{L}
$$

and

$$
t=\frac{L}{v_{0}}(1+x)=\frac{L^{2}}{\mu_{\text {oof }} V}(1+x)
$$

and if the third term is neglected, Eq. 2.19 can be rewritten as:

$$
H_{\mathrm{OT}-\mathrm{CEC}}=\left(\frac{2 \mathrm{D}_{\mathrm{m}}}{\mu}\right)+\left(\frac{x^{2}}{16(1+x)^{2}} \frac{d_{\mathrm{c}}^{2} \mu}{\mathrm{D}_{\mathrm{m}}}\right)
$$

The separation efficiency $(\boldsymbol{N})$ for any analyte is obtained from the standard deviation of its peak profile or migration zone width as in Eq. 2. 11, $N=(L / \sigma)^{2}$ and $H=L / N$. Hence, $N$ is given as

$$
N=\frac{16(1+x) \mathrm{D}_{\mathrm{m}} \mu_{\mathrm{eof}} V t}{32(1+x)^{2} \mathrm{D}_{\mathrm{m}}{ }^{2} t+x^{2} d_{\mathrm{c}}^{2} \mu_{\mathrm{eof}} V}
$$

It should be noted from Eq. 2.24 that the separation efficiency is affected by the inner diameter $\left(d_{c}\right)$ of the capillary column. It is clear that fast analysis time and efficiency can 
be achieved by using smaller diameter capillaries. However, other problems such as sample loadability and on-line detection sensitivity will suffer.

\subsection{Columns}

CEC columns are identical to those used in CE and can be either packed with HPLC stationary phase or internally coated like capillary GC columns. However, current column technology is a limitation on the advancement of the CEC technique. The historical development of the column technology and our research study in column preparations are described in Chapter 5.

\subsection{CEC Instrumentation}

As previously mentioned CEC separations are often performed on CE equipment with or without modification. The modified versions are better suited for PCC applications where it is necessary to apply pressure to both the inlet and the outlet to prevent bubble formation. Another modification is an additional HPLC pump which can be used to generate a solvent gradient by continuously refilling the electrolyte. In this research, we used a standard $\mathrm{CE}$ equipment to perform all column evaluations.

\subsection{Detection}

A variety of detectors have been employed in CEC, most commonly on-column UV detection, on-column fluorescence detection and mass spectrometry. Current applications of CEC also include CEC with laser induced fluorescence (CEC-LIF) and

electrospray ionization MS. 58 When on-column photometric detection schemes are used, 
a small optical window is fabricated by removing the polyimide coating from the fused silica column for OT-CEC and packed columns (PC-CEC). In packed capillaries, the detection window is made after the retaining frit or through the packing material.

Detection through the packing results in more efficient peaks because of focusing effects in the stationary phase at the detection window and /or elimination of band broadening associated with the retaining frit. 59,60 However, light scattering from the particles increases the noise level, resulting in lower detection sensitivity. 60,61 The design used most often in PC-CEC incorporates the detection window after the retaining frit. This would eliminate the need to connect the detection window to an open tube, which minimizes band broadening and does not restrict the system to low field strengths. Interfacing CEC with MS offers practical possibilities because surfactants need not to be added to the mobile phase and because of the sample capacity of packed capillaries. 62

\subsection{Advantages and Limitations of CEC}

\subsubsection{Advantages of CEC}

The major advantage of CEC over CE is that it can be used to separate both neutral and charged species or mixtures of the two without the addition of micelles. In this study, the author has demonstrated the use of poly(aryl ether) dendrimers as a stationary phase in OT-CEC separations of mixtures of neutral species (see Chapter 5, Section 5.6.2, Figure 5-10). CEC may offer the potential to improve selectivity for charged species as well, since the migration velocities of such compounds depend on the sum of both electrophoretic mobility and partitioning. Another advantage is that the separation of water insoluble compounds is simple with CEC because high organic content 
buffers can be used. Chiral separation applications are another advantage of CEC verses CE. Chiral selectors as stationary phase can be used in both OT-CEC and PC-CEC applications. The CEC technique eliminates the problems associated with insolubility and detection interference.

\subsubsection{Limitations}

At present, the developments in CEC technique are still deficient: (1) the column technology (both OTC and PCC), (2) the injection device, and (3) detection.

Column technology. The improvement in column technology involves two general areas: (1) stationary phase materials, (2) coating or packing techniques. The challenge is stationary phase research. There is a need for stable phases which can be used over a broad $\mathrm{pH}$ range so that the phases are not application limited. Longer column life and a simple procedure to either bond the phase to the inner capillary wall or to pack coated particles inside the capillary continue to be academic curiosities. To date, commercial availability of capillary columns is very limited, and is a serious impediment to the rapid evolution of CEC.

Injection device. Sample injection and automation need improvement. Reproducibility problems are due to the two techniques currently employed in CEC operation: (1) electrokinetic injection and (2) pressure injection. Electrokinetic injections are highly sample matrix dependent, and the use of pressure injection is limited by the high back pressure associated with pressure flow in a packed capillary. For CEC to achieve widespread acceptance within the chromatographic community and industry, it will need to prove itself to be a reliable and robust analytical technique. 
Detections. Detectors also need significant improvement. Current instruments utilize existing HPLC detectors such as UV or photodiode array, with some modifications to the detector cell dimensions and response time constants. ${ }^{63} \mathrm{UV}$ detectors do not have good detection limits due to their short cell pathlength. The $\mathrm{Z}$ cell from Hewlett-Packard offers a tenfold improvement in detection limit; however, there is a need for more improvement. 


\section{$2.8 \quad$ References for Chapter 2}

1) Compton, S.; Brownlee, R. Biotechniques 1988, 6, 432.

2) Hardy, W. B. J. Physiol 1899, 24, 288.

3) Everaerts, F. M. Graduation Report, University of Technology, Eindhoven 1964.

4) Hjerten, S. Chromatogr. Rev. 1967, 9, 122-219.

5) Mikkers, F.; Everaerts, F.; Verheggen, T. J. Chromatogr. 1979, 169, 11-12.

6) Mikkers, F.; Everaerts, F.; Verheggen, T. J. Chromatogr. 1979, 169, 1-10.

7) Jorgenson, J. W.; Lukacs, K. D. Anal. Chem. 1981, 53, 1298-1302.

8) Jorgenson, J. W.; Lukacs, K. D. Clin. Chem. 1981, 27, 1551-1553.

9) Jorgenson, J. W.; Lukacs, K. D. J. Chromatogr. 1981, 218, 209-216.

10) Jorgenson, J. W.; Lukacs, K. D. Science 1983, 222, 266-272.

11) Ewing, A. G.; Wallingford, R. A.; Olefirowicz, T. M. Anal. Chem. 1989, 61, 292A.

12) Guo, Y.; Colón, L. A. Electrophoresis 1995, 16, 493.

13) Li, S. F. Y., Capillary Electrophoresis: Principles, Practice, J. Chrom. Library 52, Elsevier: New York, 1992.

14) Cohen, A. S. Proc. Natl. Acad. Sci. 1988, 85, 9660.

15) Guttman, A. Electrophoresis 1988, 88, 151.

16) Hjerten, S.; Zhu, M. D. J. Chromatogr. 1985, 346, 265.

17) Hjerten, S. J. Chromatogr. 1987, 403, 47.

18) Xu, Y. Anal. Chem. 1995, 67, 463R.

19) Kuhn, R.; Hoffstetter-Kuhn, S. Capillary Electrophoresis: Principles and Practice, Springer-Verlag: Berlin, 1993. 
20) Terabe, S.; Otsuka, K.; Ando, T. Anal. Chem. 1985, 57, 834.

21) Vindevogel, J.; Sandra, P., Introduction to Micellar Electrokinetic Chromatography, Huthig, 1992.

22) Shaw, D. J., Electrophoresis (Academic Press, London) 1969.

23) Kuhr, W. G. Anal. Chem. 1990, 62, 403R.

24) Bartle, K. D.; Robson, M. M.; Cikalo, M. G.; Myers, P.; Euerby, M. R. 1997.

25) Bocek, P.; Kleparnik, K, J. of Chromatogr. 1991, Sept. 13, 569(1-2), 3-42. .

26) Everaerts, F. M.; Verheggen, T. P. E. M.; Aben, V. A; Reijenga, J. C. J. Chromatogr. 1983, 260, 241.

27) Virtenen, R. Acta Polytech. Scand. 1974, 123, 1.

28) Swedberg, S. A.; McManigill, D. Tech. in Protein Chem., T. Hugli ed., Academic Press: San Diego, 1989, 468.

29) Jorgenson, J. W.; Lukacs, K. D. J. High Res. Chromatogr. 1985, 8, 407-411.

30) Fujiwara, S.; Honda, S. Anal. Chem. 1987, 59, 487-490.

31) Jorgenson, J. W.; Walbroehl, Y. Anal. Chem. 1986, 58, 479.

32) Zare, R. N.; Roach, M. C.; Gozel, P. J. Chromatogr. 1988, 426, 129.

33) Tsuda, T. J. High Res. Chromatogr. Chromatogr. Commun. 1987, 10, 622.

34) Huang, X.; Luckey, J. A.; Gordon, M. J.; Zare, R. N. Anal. Chem. 1989, 61, 766.

35) Ewing, A. G.; Van Orman, B. B.; Liversidge, G. G.; McIntyre, G. L.; Olefirowicz, T. M. J. Microcolumn Sep. 1990, 2, 176.

36) Ewing, A. G.; Wallingford, R. A. Advances in Chromatography 1989, 29, 1-76.

37) Knox, J. H. Chromatographia 1988, 26, 329.

38) Knox, J. H.; Grant, I. H. Chromatographia 1987, 24, 135. 
39) Pretorius, V.; Hopkins, B. J.; Schieke, J. D. J. Chromatogr. 1974, 99, 23.

40) Knox, J. H.; Grant, I. H. Chromatographia 1991, 32, 317.

41) Smith, N. W.; Evans, M. B. Chromatographia 1994, 38, 649.

42) Lelievre, F.; Yan, C.; Zare, R. N.; Gareil, P. J. Chromatogr. A 1996, 723, 145.

43) Smith, N. W.; Evans, M. B. Chromatographia 1995, 41, 197.

44) Fang, L. Y.; Huang, E. A.; Safarpour, M. M. J. Cap. Elec.. 1998, 005: 3/4, 115.

45) Yammamoto, H.; Baumann, J.; Erni, F. J. Chromatogr. 1992, 593, 313.

46) Knox, J. H. J. Chromatogr. A 1994, 680, 3.

47) Pesek, J. J.; Matyska, M. J. J. Chromatogr. A 1996, 736, 313.

48) Vissers, J. P. C.; Claussens, H. A.; Coufal, P. J. High Resolut. Chromatogr. 1995, $18,540$.

49) Horvath, C. J.; Rathore, A. S. J. Chromatogr. A 1996, 743, 231.

50) Colón, L. A.; Guo, Y.; Fermier, A. Anal. Chem. 1997.

51) Everaerts, F. M.; van de Goor, A. A. A. M.; Verheggen, T. P. E. M.; Beckers, J. L. J. High Res. Chromatogr. 1989, 12, 28.

52) Yeung, E. S.; Pfeffer, W. D. Anal. Chem. 1990, 62, 2178.

53) Coufal, P.; Stulik, K; Claessens, H. A.; Cramers, C. A. J. High Resol. Chromatogr. 1994, 17, 325.

54) Huang, T. L.; Tsai, P.; Wu, C. T.; Lee, C. S. Anal. Chem. 1993, 65, 2887.

55) Weber, S.; Carr, P. W.; Brown, P. R.; Hartwick, R. A. 1989.

56) Giddings, J. C., Unified Separation Science, John Wiley \& Sons: New York, 1991. 
57) Bruin, G. J. M.; Tock, P. P. H.; Kraak, J. C.; Poppe, H. J. Chromatogr. 1990, $517,557-572$.

58) Lord, G. A.; Gordon, D. B.; Tetler, L. W.; Carr, C. M. J. Chromatogr. A 1995, $700,27$.

59) Rebscher, H.; Pyell, U. Chromatographia 1996, 42, 171.

60) Yan, C.; Dadoo, R.; Zhao, H.; Zare, R. N.; Rakestraw, D. J. J. Anal. Chem. 1995, $67,2026$.

61) Behnke, B.; Grom, E.; Bayer, E. J. Chromatogr., 1995, 716, 207.

62) Schmeer, K.; Behnke, B.; Bayer, E. Anal. Chem. 1995, 67, 3656.

63) Stahlberg, J. Anal. Chem., 1997, 69, 3812. 


\section{CHAPTER 3}

\section{Dendrimers}

\subsection{Introduction}

Dendrimers are well defined, highly branched macromolecules, that emanate from a central core and possess large number of terminal groups with a definite geometrical growth. Each layer of branching monomer surrounding the central core is called a "generation". Dendrimers are prepared in an iterative sequence of reaction steps, in which each additional iteration leads to a higher generation material. A schematic representation of divergent dendrimer growth from the work of Tomalia is shown in Scheme 3-1.1 These highly branched, monodisperse structures lead to a number of interesting characteristics and features, including globular, void-containing shapes and unusual physical properties such as low intrinsic viscosity, high solubility, high miscibility and high reactivity. Since their discovery twenty years ago, many different structural classes of dendritic macromolecules have been reported by several research groups. ${ }^{2-5}$ Recent advances have led to the development of a range of different methodologies for the synthesis of finely engineered dendritic structures, many of which incorporate functional moieties. Their architectural uniqueness and new properties have found an array of scientific, engineering, and clinical applications. Researchers have explored their utilization in the areas of drug delivery carriers, 6,7 chromatographic support media, 8 DNA transport structures, ${ }^{9}$ NMR contrast agents, ${ }^{10}$ molecular wires, ${ }^{6}$ and in the field of supramolecular chemistry. 11 One of the more spectacular applications is a contrast agent 
for magnetic resonance imaging (MRI) ${ }^{12}$ used to visualize the blood-stream in the body. Specific properties of dendrimers were used for this process, and this development could be at the leading edge of new applications. 12

Interest in dendritic polymers has increased nearly exponentially in the past decade. Dendritic polymers are now recognized as a fourth major class of macromolecular architectures. New applications, based on these structure-controlled architectures, have made this area the object of wide interdisciplinary interest. At the same time, little is known about the three-dimentional structure and the interior nanoenviroment of dendrimers.

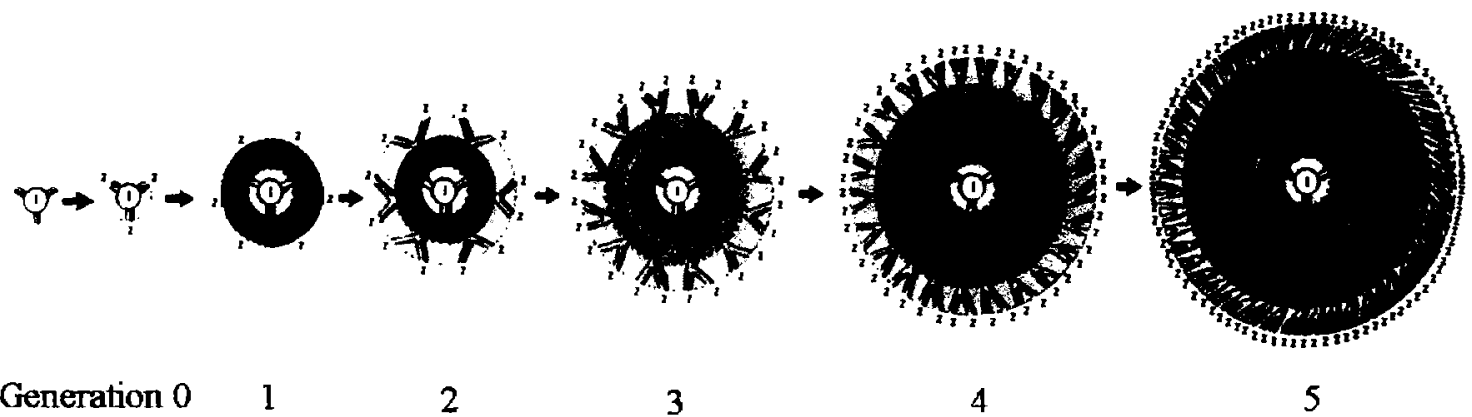

Scheme 3-1. Tomalia's schematical representation of the divergent dendrimer growth. ${ }^{1}$ 


\subsection{Historical}

Dendritic polymers are defined as polymers with a three dimensional, tree-like, branched structure. However, they can be considered a novel class of hyperbranched polymers, three dimensional structures known since the 1930 s. ${ }^{13,14}$ Flory 15,16 was the first to provide both experimental and theoretical evidence for the formation of branchedchain, three dimensional macromolecules in polymerizations. In the past 20 years, researchers have shown increasing interest in the design and synthesis of hyperbranched polymers. By the end of the 1970s, interest was increasing in the new areas of host-guest (Figure 3-1) 17-19 and supramolecular (Figure 3-2) 20-23 chemistry. Large molecular compounds, such as "octopus"24-26 and "tentacle"27 molecules, were synthesized. They were found to act as selective hosts or catalysts despite their highly flexible structures. The quest for other molecular receptors increasingly interested many researchers.

The concept of cascade molecules was first introduced by Vogtle in 1978 and developed during the mid 1980s and early 1990 's, by Tomalia, Newkome, Fréchet and others. Tomalia's group 4,28-30 accomplished a "stepwise polymerization" route which gave birth to the polyamidoamine (PAMAM), "starburst dendrimers", (Figure 3-3). These highly branched polymers with extremely low polydispersities were reported to 10 generations with yields on the order of $98-100 \%$ at every step.

In 1985, Newkome's group pioneered an alternative type of amplification chemistry to produce treelike molecules, which they referred to as "arborols".5,31,32 The arborol's branching was derived from tetrahedral carbon atoms where each layer (or generation) had a different constitution - leading to very compact "arborol" structure. 
Examples of Newkome's very highly branched "arborols" (Figure 3-4) which were named because of their surface hydroxyl groups and the Greek word arbor, meaning tree. these molecules were functionalized with polar surface groups and investigated as covalently linked micelle analogues and as supramolecular building blocks.

Another interesting approach to dendrimer synthesis was described in 1989 by Jean M. Fréchet and Craig J. Hawker ${ }^{33}$ of Cornell University and separately, in 1990 by Timothy M. Miller and Thomas X. Neenan ${ }^{34}$ of AT\&T Bell laboratories. Instead of using the so-called divergent method - starting from the inside and building outward - as for Tomalia's PAMAM and Newkome's arborol, they developed what is known today as the convergent method of synthesis. They constructed individual branches first and then attached the units to a central core molecule. Finally, Moore's convergently produced phenylacetylene dendrimers are the last of the five classes of dendrimers that are most well known. 


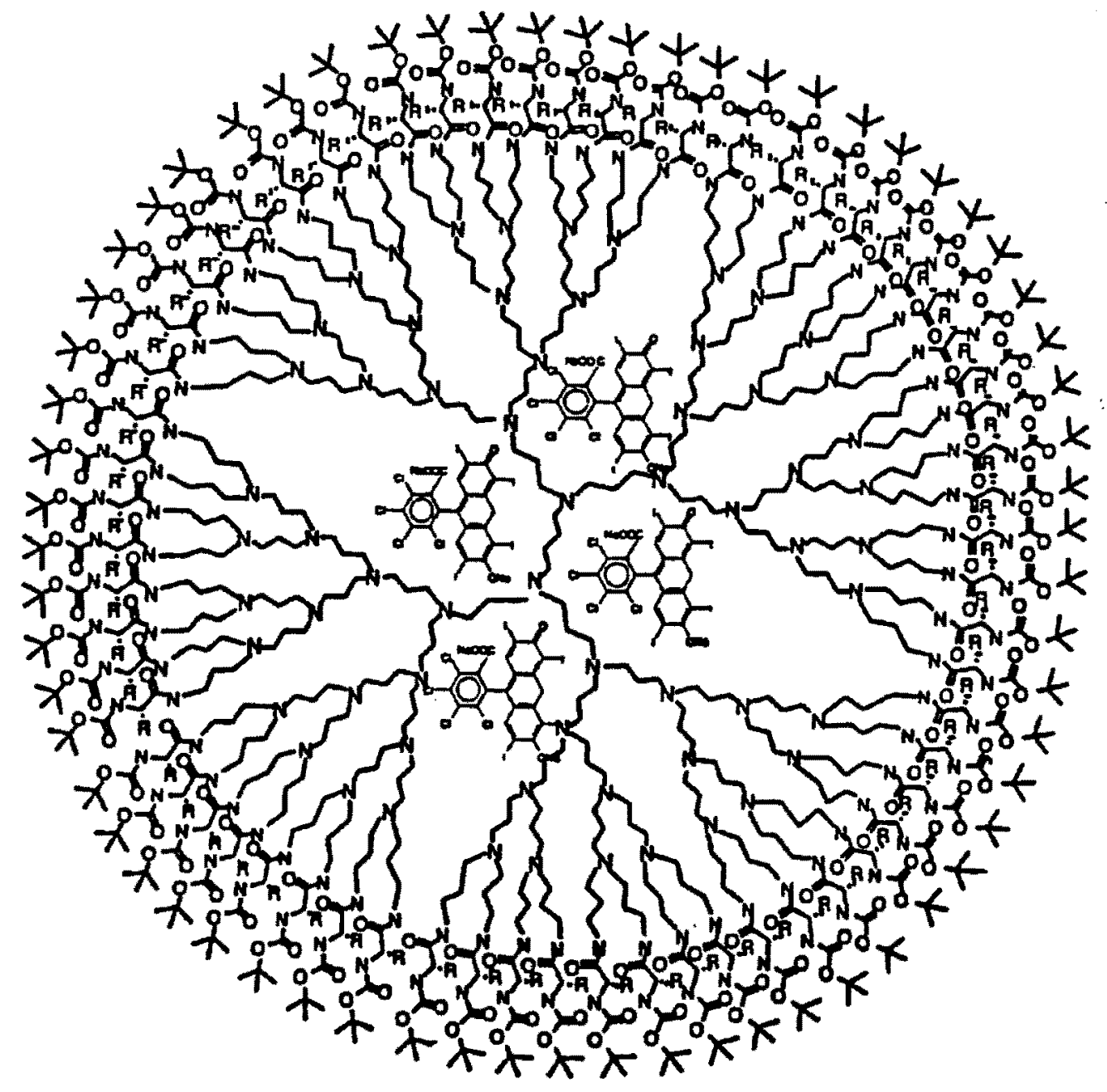

Figure 3-1. Dendritic box hosts guest molecules in its cavities. 35 

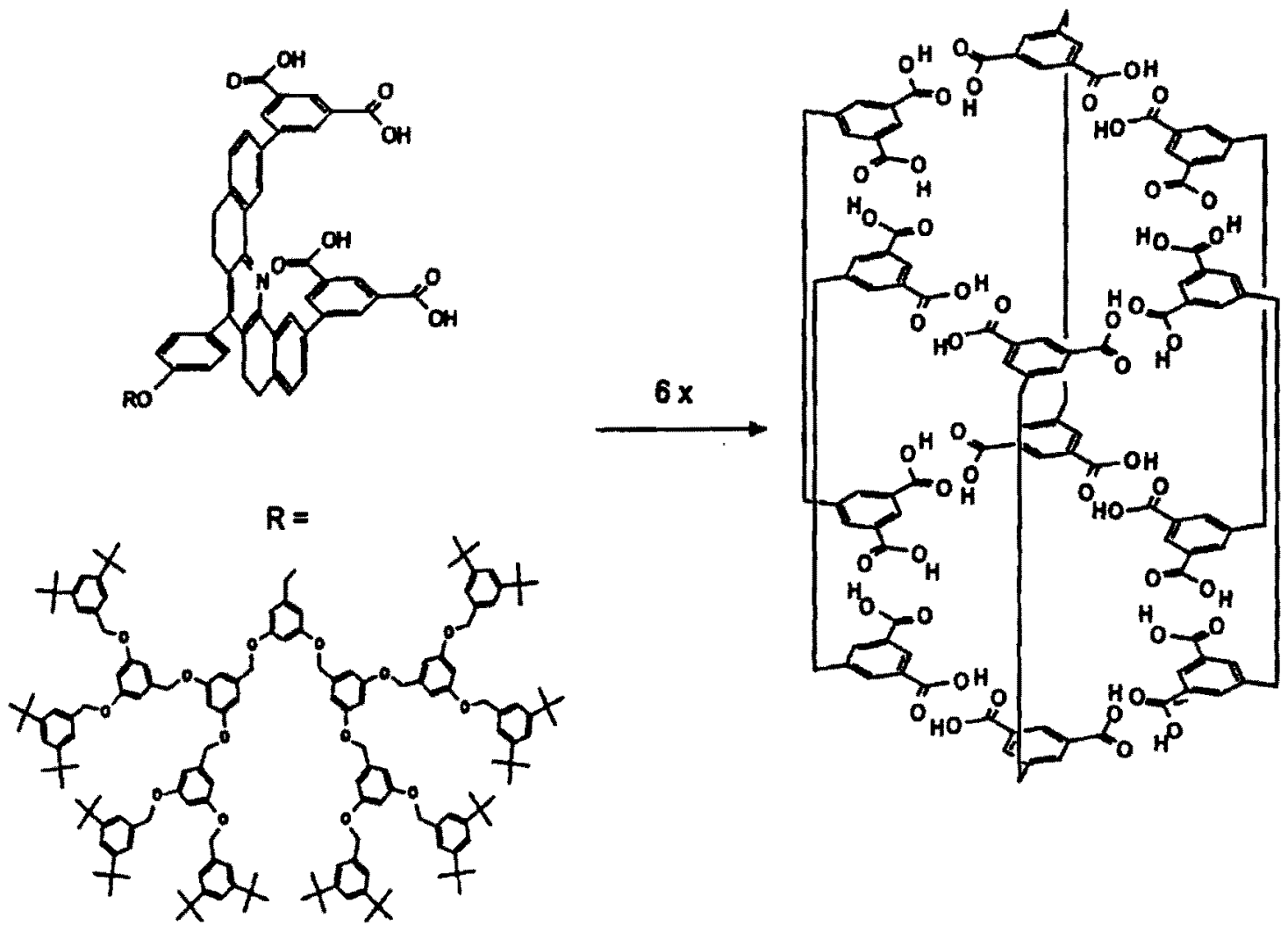

Figure 3-2. Supramolecular arrangement to form a hexameric dendrimer. ${ }^{36}$ 


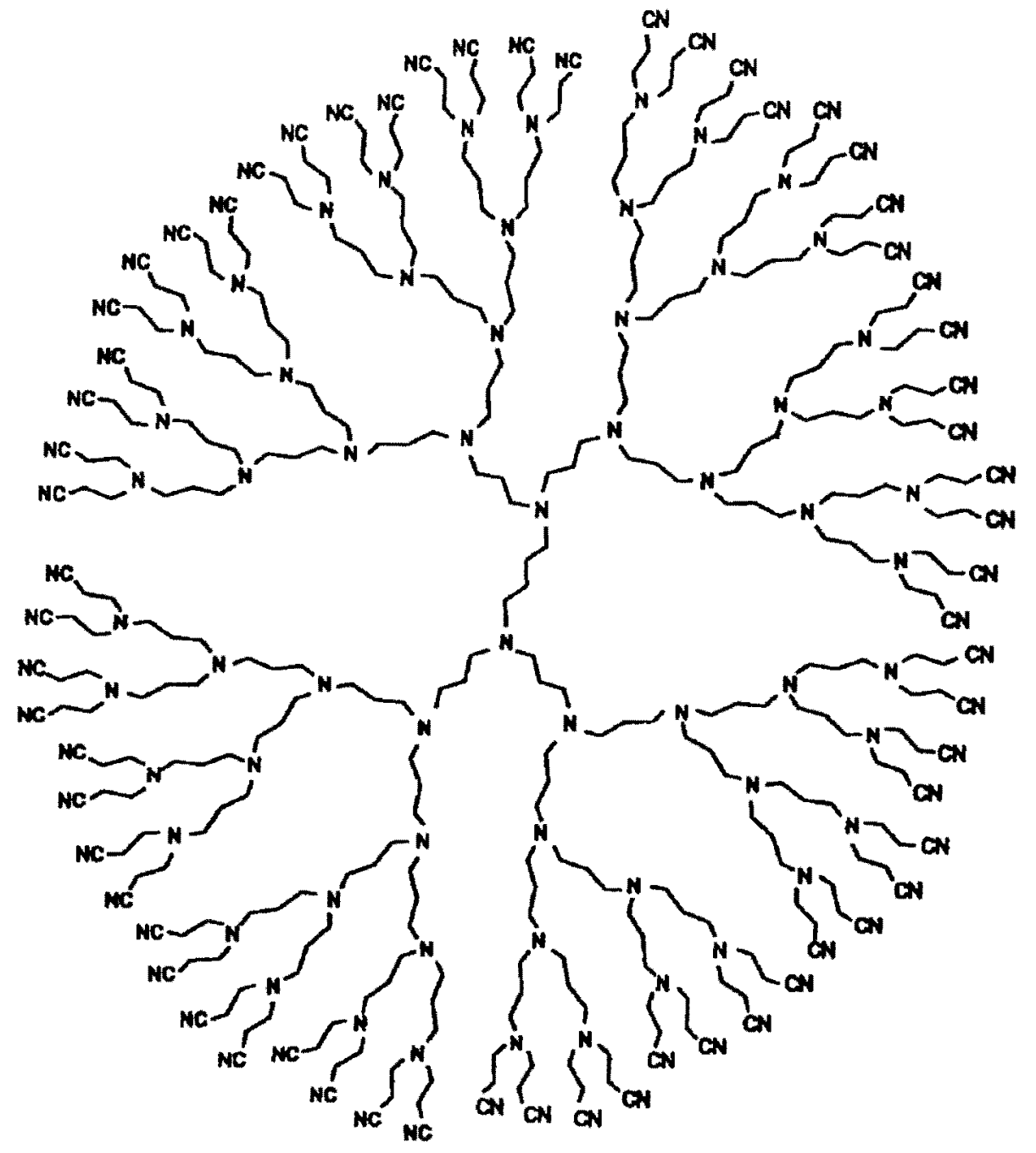

Figure 3-3. Starburst dendrimer, poly(propylene imine) dendrimers. 37 




Figure 3-4. Arborol dendrimer, 27 cascade: hexane[3-1,1,1]: (4-oxapentylidene) : (3oxo 2-azapropylidene): methanol. 37 
While these synthetic developments were occurring, numerous other researchers were investigating the structure and the physical and chemical properties of these novel macromolecules. Aharoni and co-workers ${ }^{38}$ studied Denkewalter's lysine-based cascade dendrimers ${ }^{3}$ using viscosity determinations, photocorrelation spectroscopy (PCS), and size-exclusion chromatography (SEC). They reported that at each step the polymers were monodisperse and behaved as nondraining spheres. Several theoretical investigations were performed to elucidate the topology and chemistry of the surface and cavities of dendritic polymers. De Gennes and Hervet ${ }^{39}$ predicted that the external surface of a molecular tree becomes saturated because of dense packing when it reaches a given number of generations. This depends on the topology and the length of the segment added at each generation. If the construction is continued beyond this point, defects start to appear and the molecular object is no longer well defined. In this model, the authors assumed that the end groups of the dendrimer are grouped in concentric circles around the core. A selfconsistent field (SCF) model predicts a density minimum at the center and limiting polymer size due to steric congestion. Muthukumar and Lescanec 40 on the other hand, used a computer simulation based on a kinetic growth model to predict a monotonic decrease in density on going from the center of the dendrimer to its periphery. In this model, the ends of the branches are not positioned at the surface but are severely backfolded (Figure 3-5). 


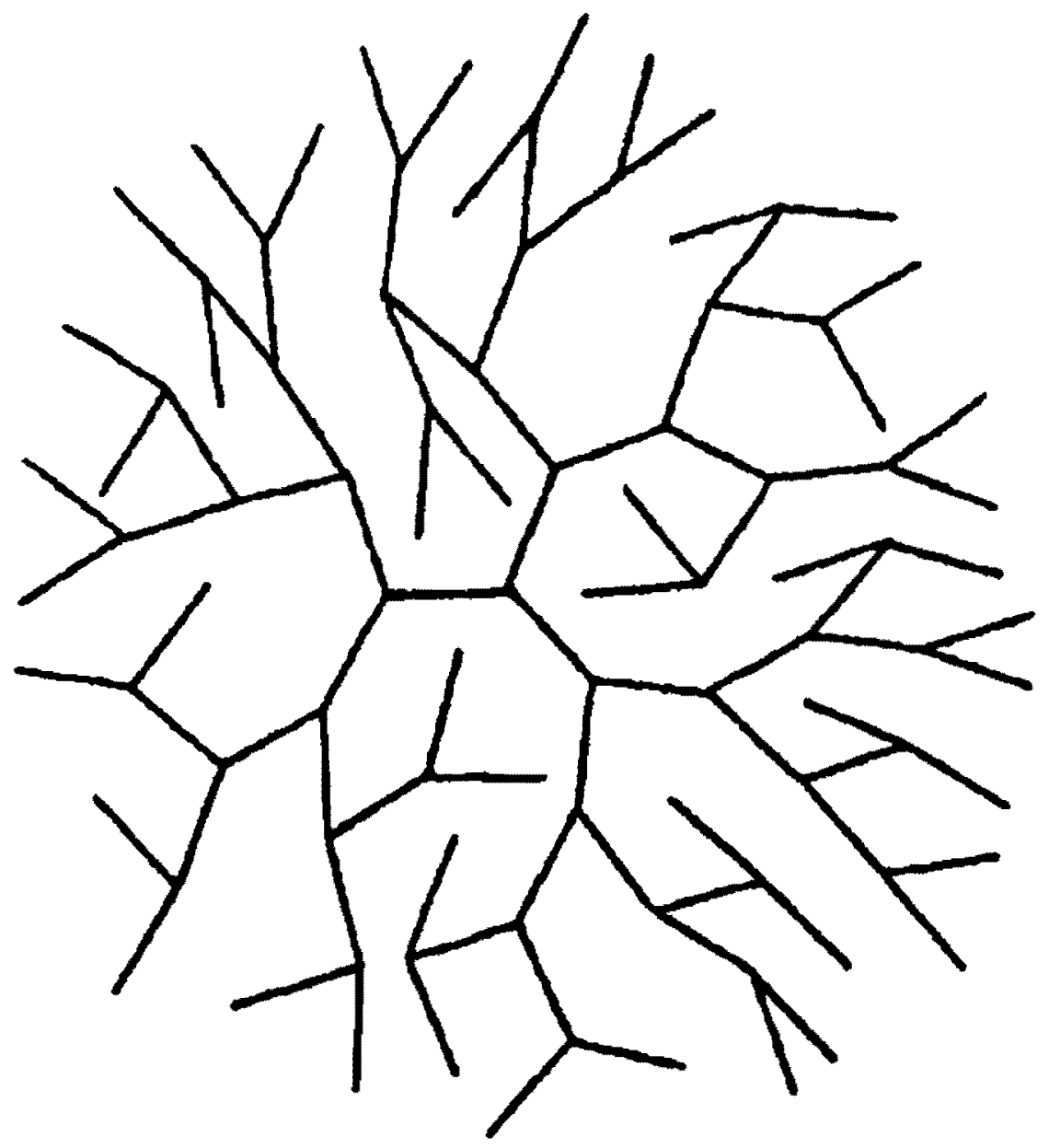

Figure 3-5. Schematic representation of a backfolded trifunctional fifth generation dendrimer according to Boris and Rubinstein. 41 
The conformational behavior of dendrimers is still an issue of debate. Are the end groups pointing outward or are they backfolded? Do dendrimers always have a globular shape or can this shape be highly distorted? Are cavities present inside the dendrimer? Recent theoretical studies by numerous investigators have indicated that backfolding is an important process in most models. A molecular dynamics (MD) study of Murat and Grest ${ }^{42}$ has shown a strong correlation between the solvent polarity and the mean radius of gyration. Muthukumar and Welch, ${ }^{43}$ using Monte Carlo simulations on dendritic polyelectrolytes, show a dramatic change in dendrimer conformation depending on the ionic strength of the solvent. At high ionic strength, backfolding of the end groups takes place and a "dense core" dendritic structure is formed. At low ionic strength, the multiple charges in the dendrimer force the molecule to expand, resulting in a "dense shell" structure. Nevertheless, many studies show that the importance of backfolding is dependent on the actual dendritic structure. Attractive secondary interactions between the end groups can effect the conformations of the branches significantly, thereby notably reducing backfolding (Figure 3-6). 


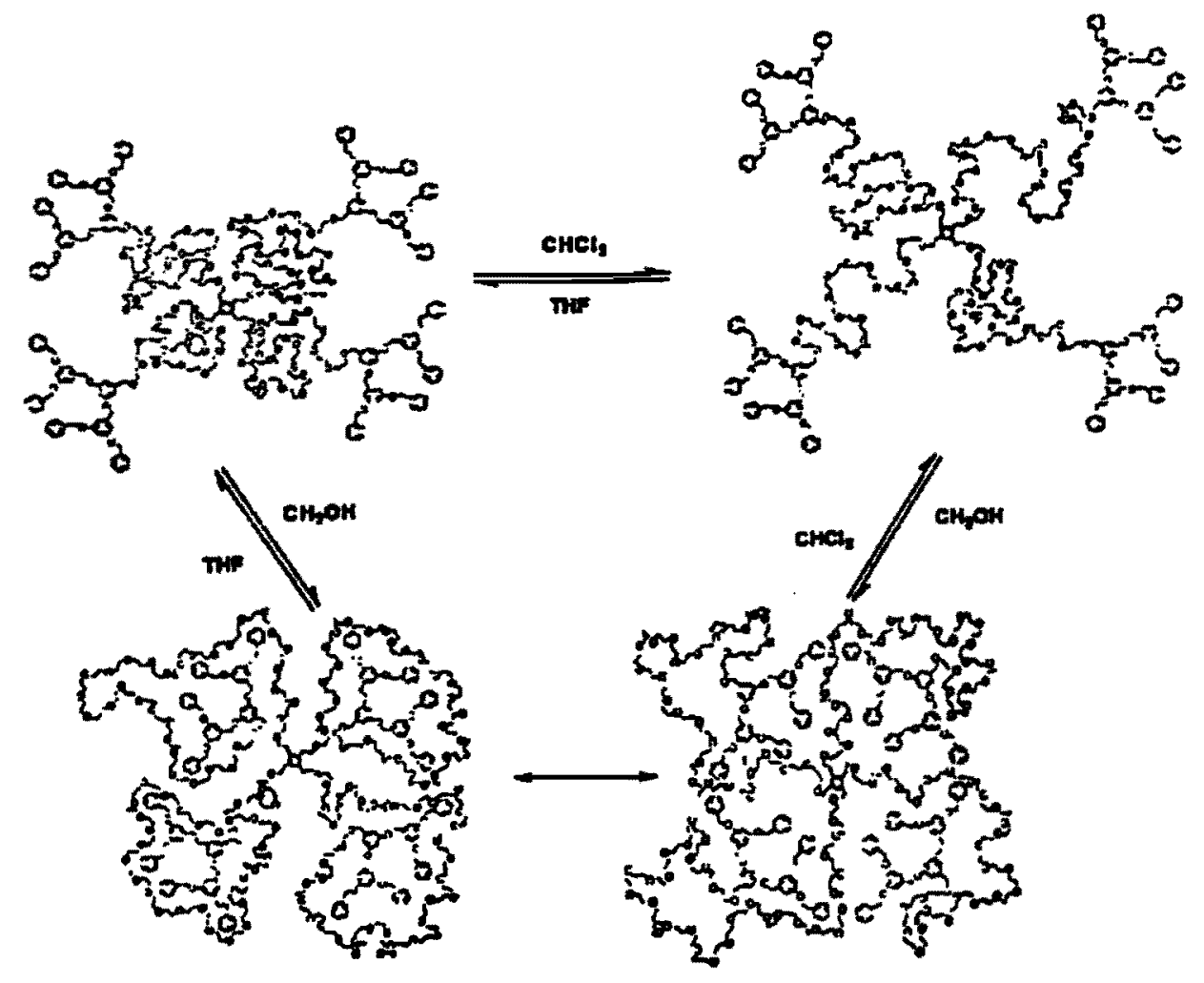

Figure 3-6. Dendritic copolymer responds to changes in its environment. 44 


\subsection{Synthesis}

Two main synthetic methodologies for synthesizing dendrimers have emerged over the past decade: the divergent and convergent approaches (Scheme 3-2).

A)
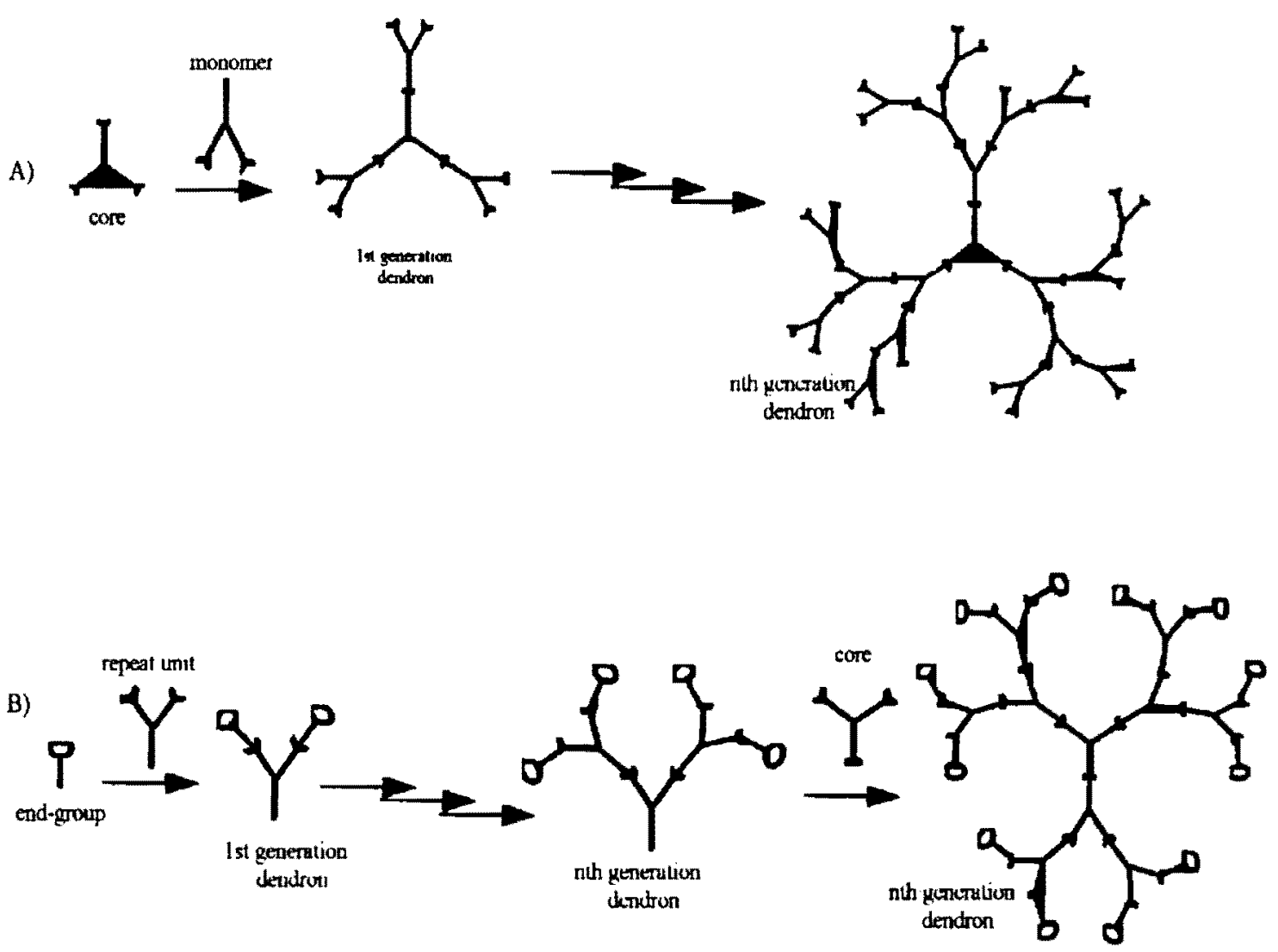

Scheme 3-2. A) Divergent preparation of dendrimer, B) Convergent preparation of dendrimer. ${ }^{45}$ 
Both approaches consist of a repetition of reaction steps, each repetition accounting for the creation of an additional generation. In the divergent synthesis, the dendrimer is grown in a stepwise manner from a central core, implying that numerous reactions have to be performed on a single molecule. Consequently, every reaction has to be highly selective to ensure the integrity of the final product. The divergent synthesis can be seen as the macromolecular approach toward dendrimers: the purity of the dendrimers is governed by statistics. An example of the divergent synthesis scheme is shown in Scheme 3-3. In contrast to the divergent strategy, the convergent approach builds the dendrimer from the periphery toward the central core. Scheme 3-4 depicts the convergent synthesis route. Thus, the initial reaction sites ultimately reside on the periphery of dendrimer, while the final reactions take place at the reactive core, also called the focal point or focus. Another key difference is that the number of coupling reactions needed to add each new generation, depending on branch multiplicity, is constant and a low number of reaction sites is assured in every reaction step throughout the synthesis, resulting in dendrimers of higher purity. Dendrimers prepared by the convergent strategy are generally considered to be more homogeneous than those prepared by divergent approach, since defects tend to accumulate at higher generation numbers when a large number of coupling or condensation reactions have to occur on a congested dendrimer surface. On the other hand, the convergent strategy is often limited to dendrimers of lower generation numbers (6 or lower). Dendritic wedges of higher generations encounter serious steric problems in reactions associated with their "focal points", and have been found to be inappropriate for the construction of dendrimers close to their dense packed starburst limit. 

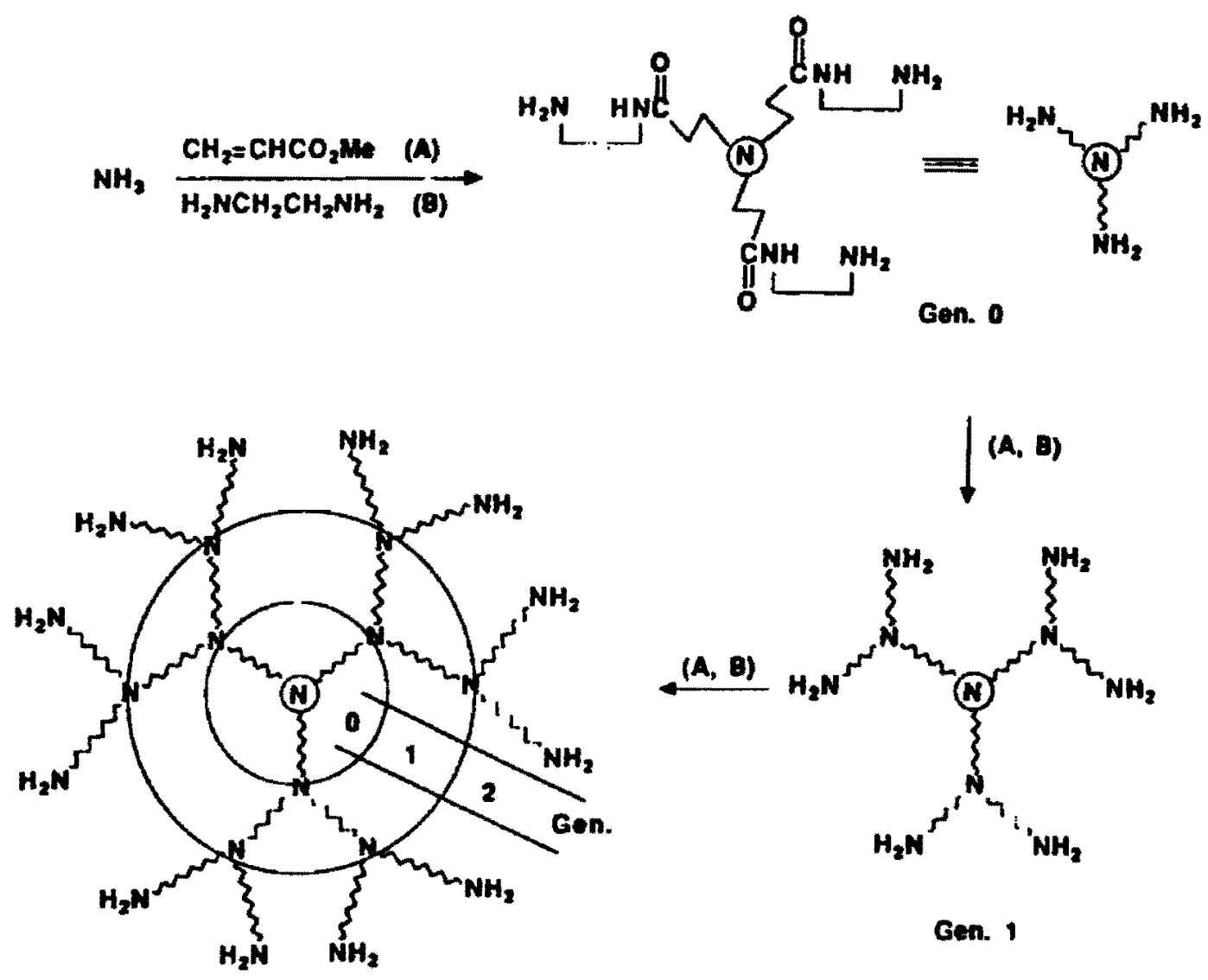

Scheme 3-3. Synthetic scheme for starburst PAMAM dendrimers. ${ }^{1}$ 


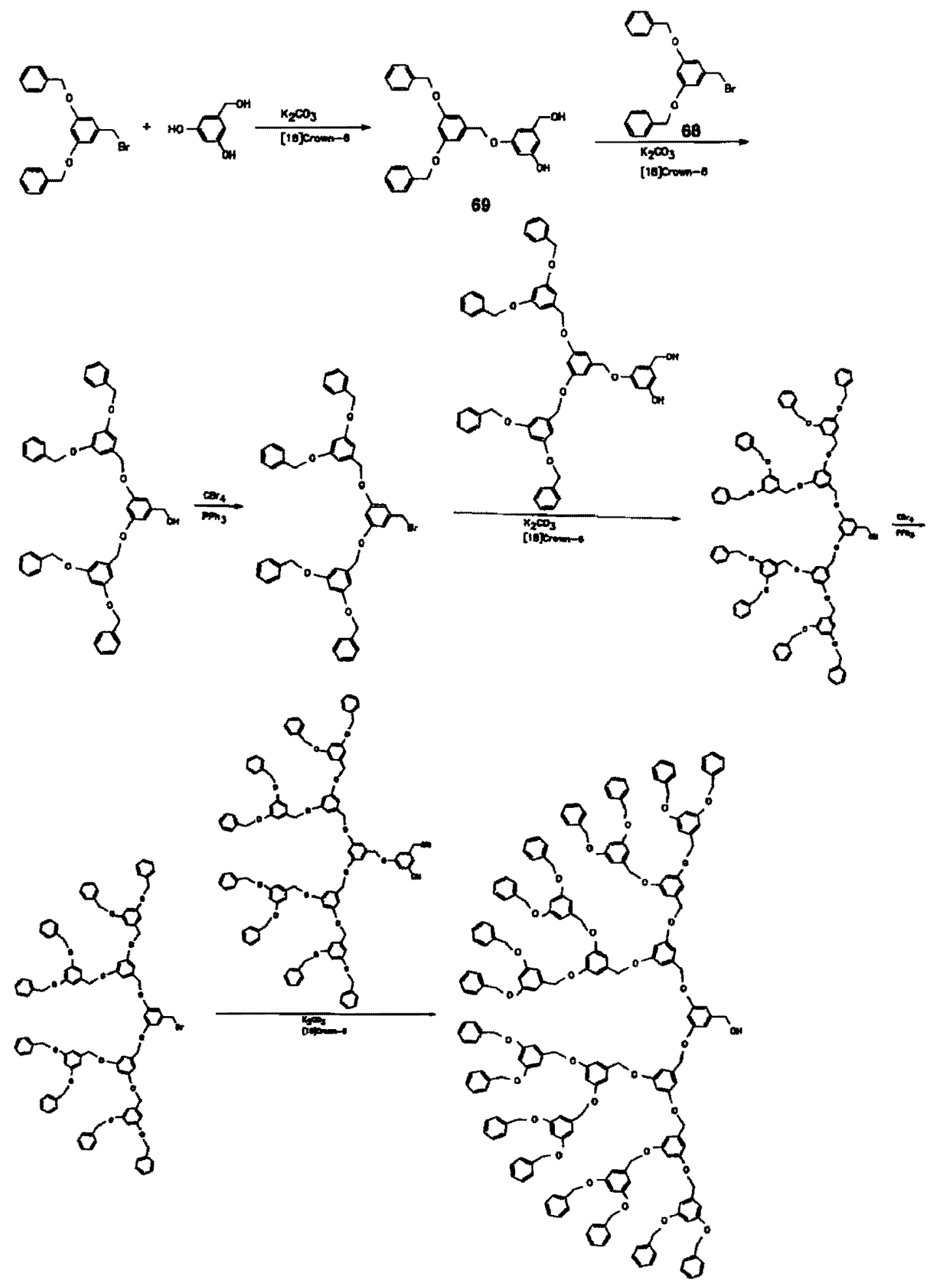

Scheme 3-4. Convergent preparation of poly(aryl ether) dendron. 46 
The limits of both approaches have yet to be firmly established, but at this time the divergent approach has been favored for potential commercial scale development, for example Astromol, poly(propylene imine) (PPI) and Starburst, poly(amidoamine) (PAMAM) dendrimers. The convergent approach has been widely adopted for laboratory syntheses. However, the limitation of relative small scale dendrimer syntheses due to the inherent difficulty of the chemistry involved was recently challenged by Rannard and Davis. 47 The authors reported that the highly selective reactions of $1,1^{\prime}$-carbonyl diimidazole (CDI) with amines, alcohols and carboxylic acids allows the preparation of a variety of highly functionalised dendritic structures on a relatively large scale. It has been demonstrated that the intermediate imidazole derivatives show high selectivity in subsequent reactions with amines and alcohols. Their results indicated that it is possible to design syntheses in which protection-deprotection chemistry is not required (Scheme 3-5).
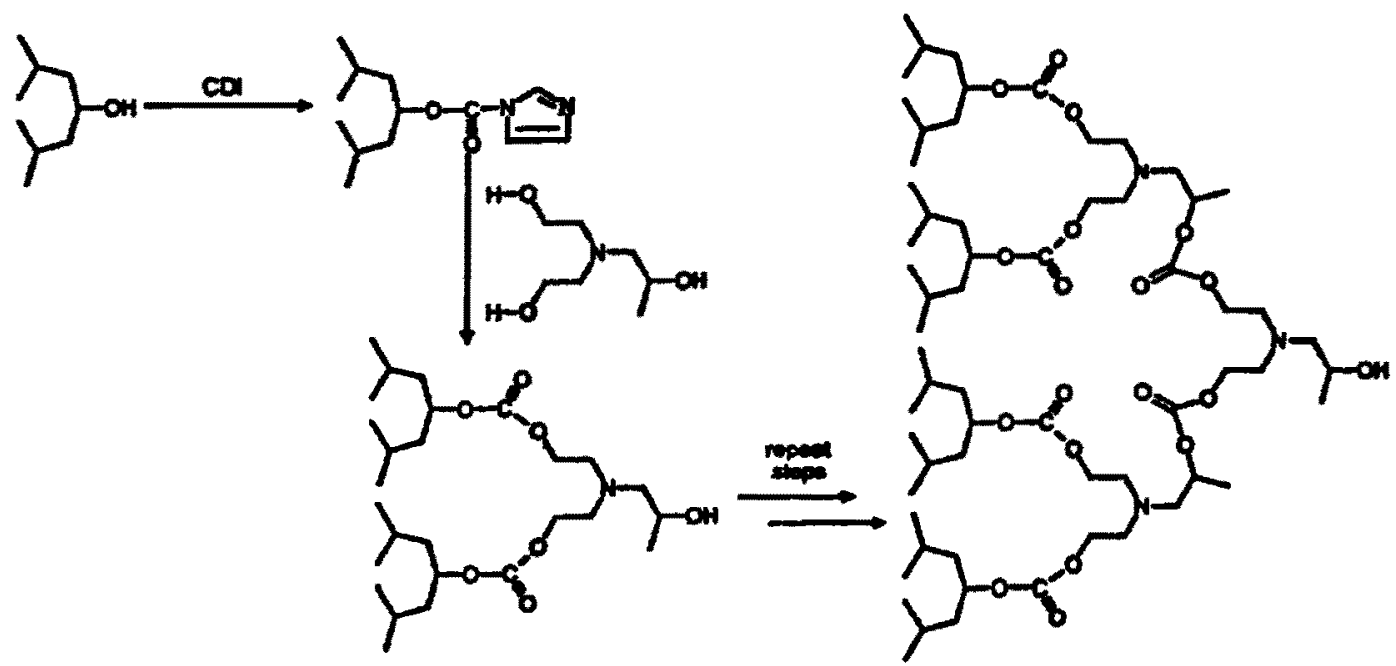

Scheme 3-5. Synthetic scheme using the CDI chemistry. 47 
The search for synthetic methods which would enable rapid amplification of dendrimer structures to higher molecular weights with fewer reiterative process steps prompted researchers to look for other alternate route. Moore and co-workers 48 examined a so-called double exponential dendritic growth process (Scheme 3-6).
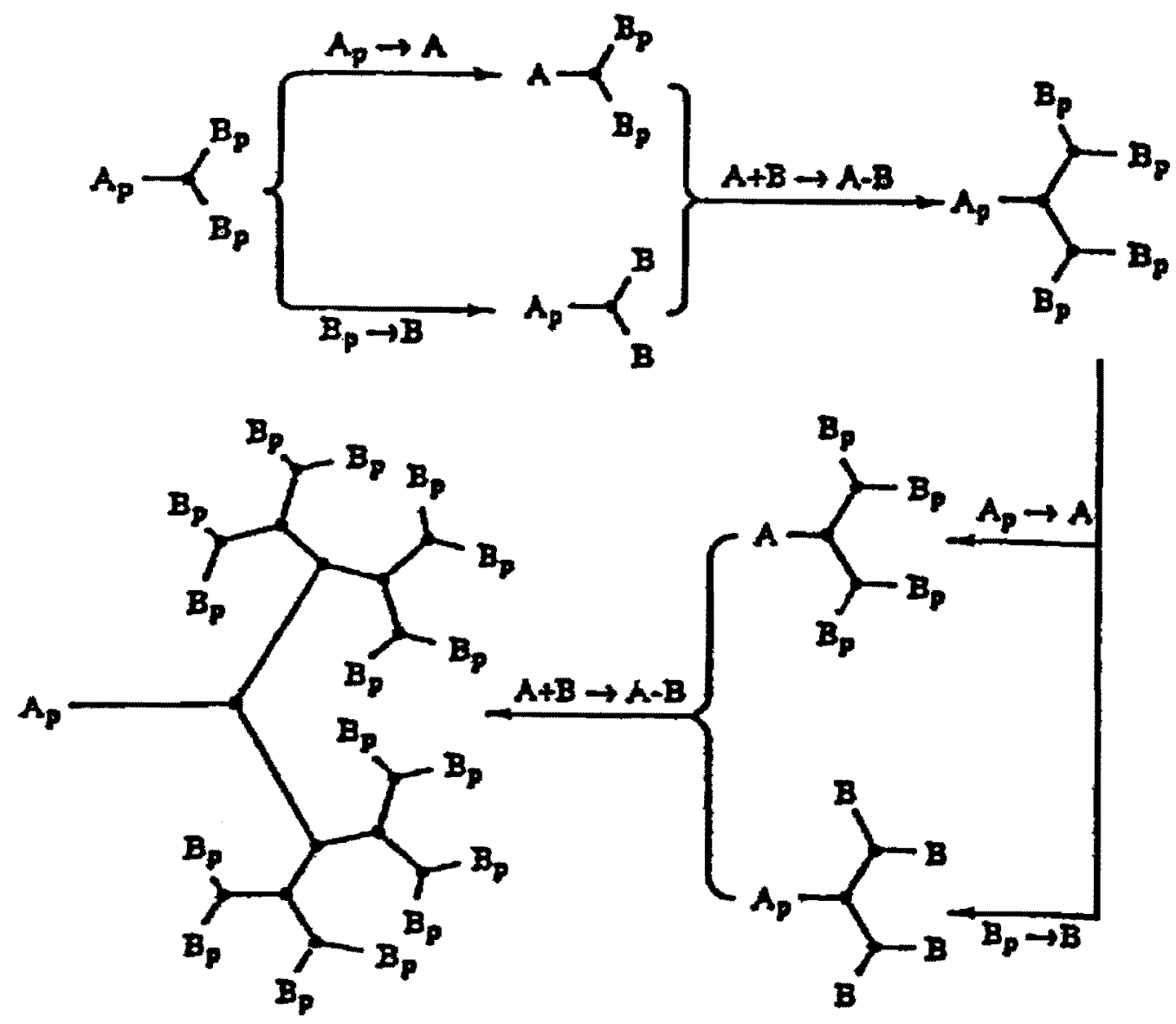

Scheme 3-6. The double exponential growth process. 48 
In this convergent process, the degree of polymerization increases with generation number (n) so that the overall molecular weight of the resulting dendron molecule increases $\left(2^{n}-n\right)$ times faster than for classical convergent growth. Their poly(phenylacetylene) dendrimers represent molecular mass 'record holders' for pure hydrocarbons, with molecular masses as high as 40,000 amu. A related approach to more rapid dendrimer growth was also reported by Fréchet et al. and involved so-called hypermonomers. ${ }^{49-51}$ This method involves the pre-assembly of oligomeric species, which can then be linked together to give dendrimers in fewer steps or higher yields. Another advance made in the area of fast dendrimer growth is the "orthogonal" approach developed by Spindler and Fréchet. ${ }^{52}$ In this approach, two different monomers are used so as to avoid the need for an activation step between growth steps. The two monomers are of constitutions $A B_{2}$ and $C D_{2}$, where $A$ and $D$ react to form a bond under conditions where $\mathrm{B}$ and $\mathrm{C}$ are stable, and vice verse for $\mathrm{B}$ and $\mathrm{C}$. Convergent or divergent growth is theoretically possible using this method. An example to illustrate Fréchet's orthogonal growth process is shown in Scheme 3-6.

In all cases the dendrimer skeleton is built up in a repetitive manner, namely, after one reaction a further step for the deprotection or activation has to be carried out. The "orthogonal coupling" method is an effective synthesis that alternately uses two different branch cells with complementary coupling functionalities. 53 For this strategy, the functionalities and the coupling products have to be inert under the reaction conditions of the second functionality pair. This way the dendrimer skeleton can be constructed either divergently or convergently depending on the target molecule. Bradley et al. ${ }^{54}$ reported a 
solid-phase synthesis analogous to the Merrifield peptide synthesis. This method allows the use of a large excess of reagents to achieve complete reaction without causing any purification problems. By connecting a group that was unstable to acid to the resin, the separation was guaranteed, and it was possible to synthesize a polyamide dendrimer up to the fourth generation.

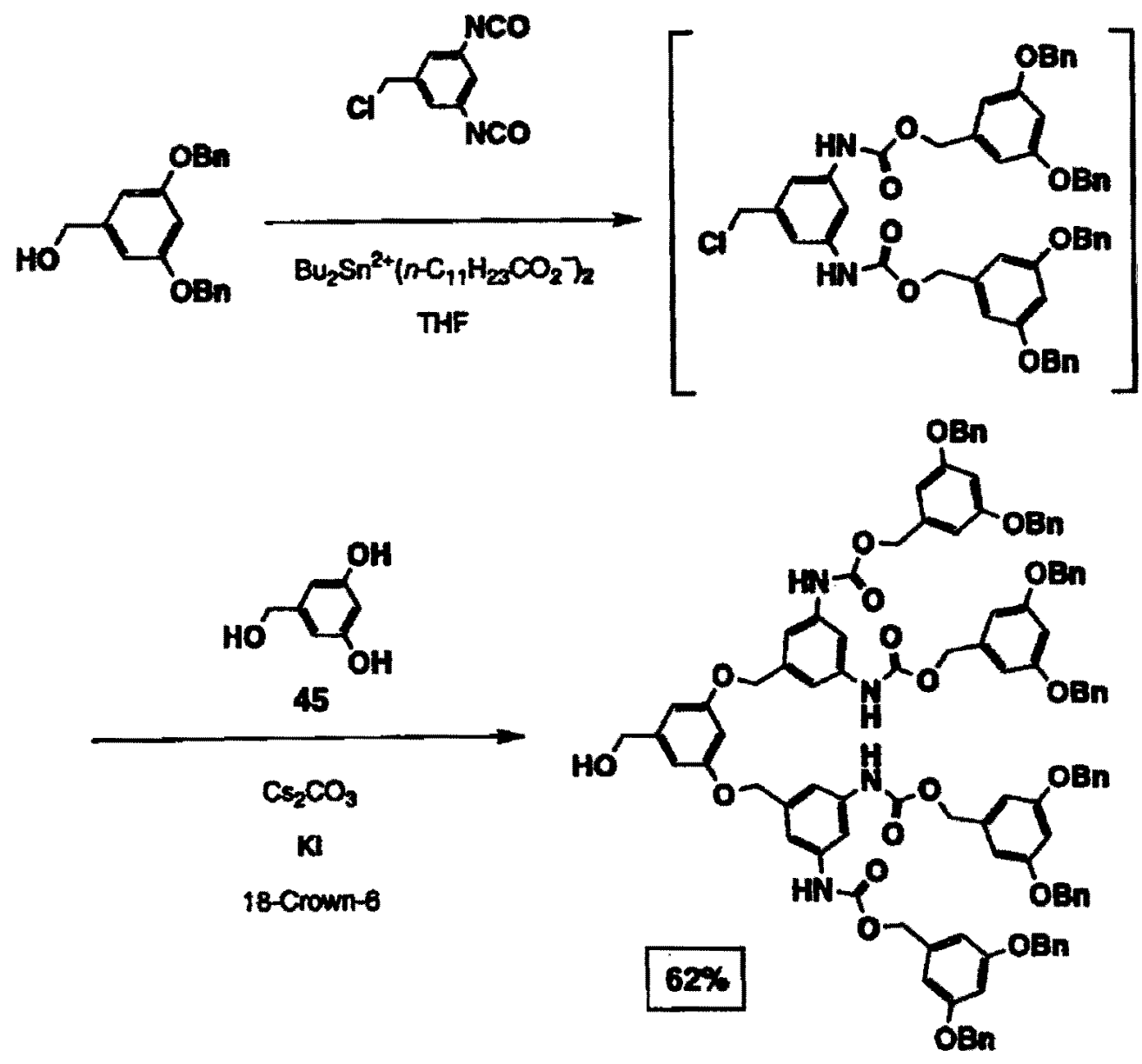

Scheme 3-6. Synthetic scheme illustrates Fréchet's orthogonal growth process. 52 
Synthesis of new dendrimer families containing main group elements and metals is another active area of research. Thus, following earlier preparations of polysiloxane and polycarbosilane dendrimers, 55,56 three different research groups reported the synthesis of new polysilane dendrimers in 1995. ${ }^{57-59}$ In all cases divergent synthesis methods were used based on halogen-metal reactions of chloro- and lithiosilanes, leading to Si-Si bond formations. The highest molecular weight product obtained contains $31 \mathrm{Si}$ atoms, and 63 $\mathrm{CH}_{3}$ groups with molecular masses equal to $1,833 \mathrm{amu}$ at generation two. 59 This synthesis used chlorination of the methylsilane or phenylsilane intermediates resulting from the halogen-metal interconversion reaction with trifluoromethanesulfonic acid in dichloromethane. 59 It was suggested 58 that polysilane dendrimers may show improved Si-Si bond stability relative to their linear counterparts, while still retaining their highly desirable optical and electronic properties.

Initially, research activity in the dendritic polymer field focused on the synthesis of new dendrimer families with an emphasis on structure amplification to higher molecular weights by advancement through many generations, Recently the research direction has clearly changed, so that process enhancement, chemical modifications, physical characterization and applications of dendrimers have received more attention. Most chemical modifications reported have involved dendrimer surfaces; however, recent examples include interior modifications.

Several new phosphorus-containing dendrimers were reported by Majoral and coworkers. 60,61 These authors developed an effective divergent synthesis of dendrimeric polyaminophosphines using $\mathrm{S}=\mathrm{PCl}_{3}$ as an initiator core reagent. 60 This dendrimer family 
was advanced to the seventh generation ( $384 \mathrm{P}-\mathrm{Cl}$ surface groups and a molecular mass of 94,146 amu), after which the highly reactive surface groups were further modified. A unique modification with bis-allylamine, was reported to give monosubstituted mixed surface products only. This is the first example of a dendrimer possessing two different functional groups at each terminal site. They have also shown that the preparation of particularly high generations (up to generation ten) is possible based on divalent phosphorus branch junctures. 62 By coupling the $10^{\text {th }}$ generation (3072 end groups) with a diphenylphosphanyl unit and complexing gold (I) chloride, they have built a complex that contains up to 3,072 gold ions; the size of the complex has been determined by high resolution electron transmission microscopy to be about 15 nanometers $(150 \AA)$. Correspondingly, they managed to synthesize multipalladium, -platinum, and rhodium complexes by grafting diphosphane units onto lower generations of the dendrimer. ${ }^{63}$ Very recently the same group even succeeded in divergently building up new dendrons inside the skeleton. 64

New divergent synthetic process involving metal-ligand complexation strategies have led to unique dendrimer structures exhibiting both luminescent and redox-active properties. Extensive efforts by an Italian research team ${ }^{65}$ have produced dendrimer structures possessing as many as 22 metal centers within their interiors. Related unimolecular dendrimer devices reported by Xu and Moore ${ }^{66}$ may result in the possibility of spatially manupulating excited state energy and /or charges by means of a convergent directional molecular antenna effect. 
Dendrimer surface modifications have evolved in two major directions: firstly, reactions involving low molecular weight reagents to give subnanoscopic modifications, and secondly, reactions involving synthetic or biological macromolecules, leading to nanoscopic modifications.

Several preparations of dendrimers with organometallic groups at the periphery have been reported.67 A silicon-based dendrimer functionalised with up to 12 catalytically active nickel or palladium complexes at the periphery was reported by Van Koten and Jastrzebsli. Liao and Moss ${ }^{68}$ prepared an organoruthenium dendrimer with up to 48 metal centres at the periphery using a convergent synthesis based on a ruthenium functionalised wedge (Figures 3-7) and the Fréchet metholodgy. Two other PPI dendrimer periphery modifications were reported separately. Cuadrado et al. ${ }^{69}$ reported that the reaction of 1-(chlorocarbonyl) ferrocene with the dendrimers terminal amine groups can introduce up to 64 ferrocene groups on the periphery. The complete conversion was supported by NMR and IR spectroscopy. Meijer and coworkers 70 used PPI dendrimers for the site-specific complexation of copper (II), zinc(II) and nickel (II) chlorides, and have functionalised the dendrimer periphery with up to 32 metal groups. Analytical methodologies such as visible, NMR and ESR spectroscopy were used to characterize the complexation of M(II) transition metal ions with the terminal groups of poly(propylene imine) dendrimers. These are of interest as catalysts as they combine high solubility with easy recovery by dialysis or ultrafiltration techniques, thus combining the advantages of conventional homogeneous catalysts and polymer supported reagents. 


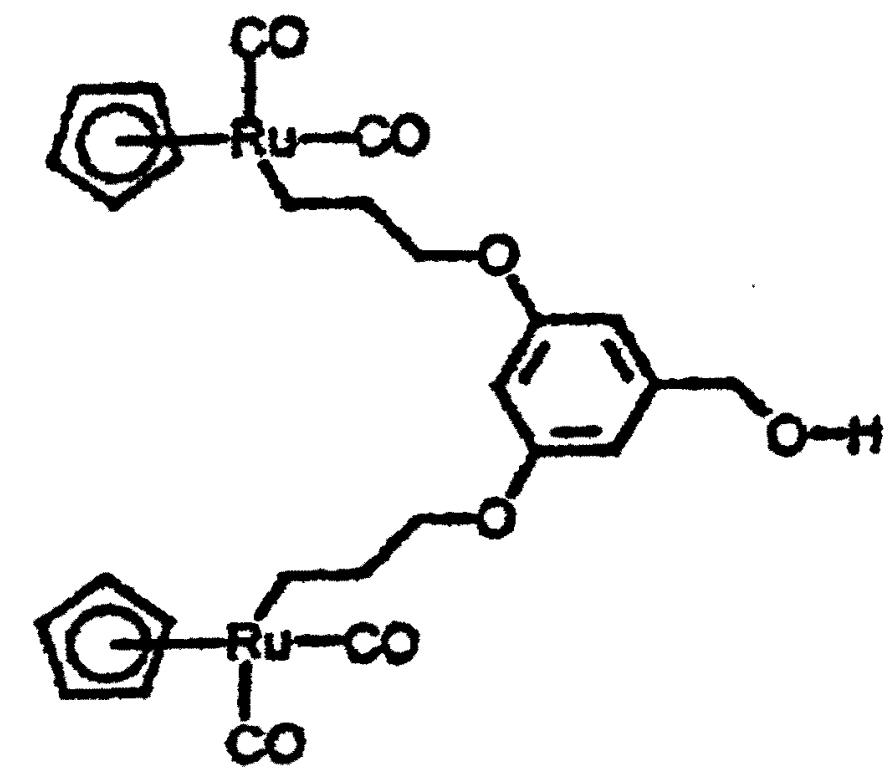

Figure 3-7. Liao and Moss's organoruthenium dendrimers. 68

The assembly of dendritic wedges into larger structures has been investigated by several groups. Tzalis and Tor 71 have prepared first generation dendritic wedges with phenanthroline ligands at the focus, and has demonstrated their self-assembly around metal centers. Replacing the focal ligand with groups that form strong hydrogen-bonds is an alternative approach to self-assembled dendrimers. Zimmerman's 72,73 work describes poly(aryl ether) dendrimers functionalised at the focus with isophthalic acid groups in an orientation that allows the formation of discrete hexameric self-assembled structures. 
Experimental evidence suggests that the hexameric structures are formed, with the stability of the aggregates dependent on the solvent and size of the dendritic wedge.

Perhaps, the most noteworthy dendrimer modification is the construction of the "dendritic box" prepared by Meijer and co-workers at the University of Eindhoven. They have enhanced an earlier concept for producing artificial cells by modifying dendrimer surfaces to introduce "unimolecular encapsulation" behavior. Surface-modifying generation 5 PPI dendrimers with 1-phenylalanine or other amino acids induced dendrimer encapsulation by forming dense, hydrogen-bonded surface shells with almost solid state character. Small guest molecules were captured in such dendrimer interiors and were unable to escape even after extensive dialysis. 74,75 The maximum number of entrapped guest molecules per dendrimer box is determined by the shape and size of cavities and guests. Meijer et al. has demonstrated the encapsulation of four large molecules and 8-10 smaller molecules simultaneously within these PPI dendrimers. Shape-selective liberation of guests with different sizes is also achieved by the hydrolysis of the shell in a two-step process. First, partial hydrolysis of the hydrogen-bonded shell liberated only small guest molecules (p-nitrophenol, p-nitro-benzoic acid or an EPR probe). The larger guests (bengal rose, rhodamine, new coccine and methylene violet 3RAX) are liberated by subsequent hydrolysis (with $12 \mathrm{~N} \mathrm{HCl} ; 2$ hours at reflux) of the amide groups. This remarkable study has shown that it is possible to tune the shape-selectivity in the liberation of guests of different size by changing the amino acid and the protected groups used (Figure 3-8). 35 


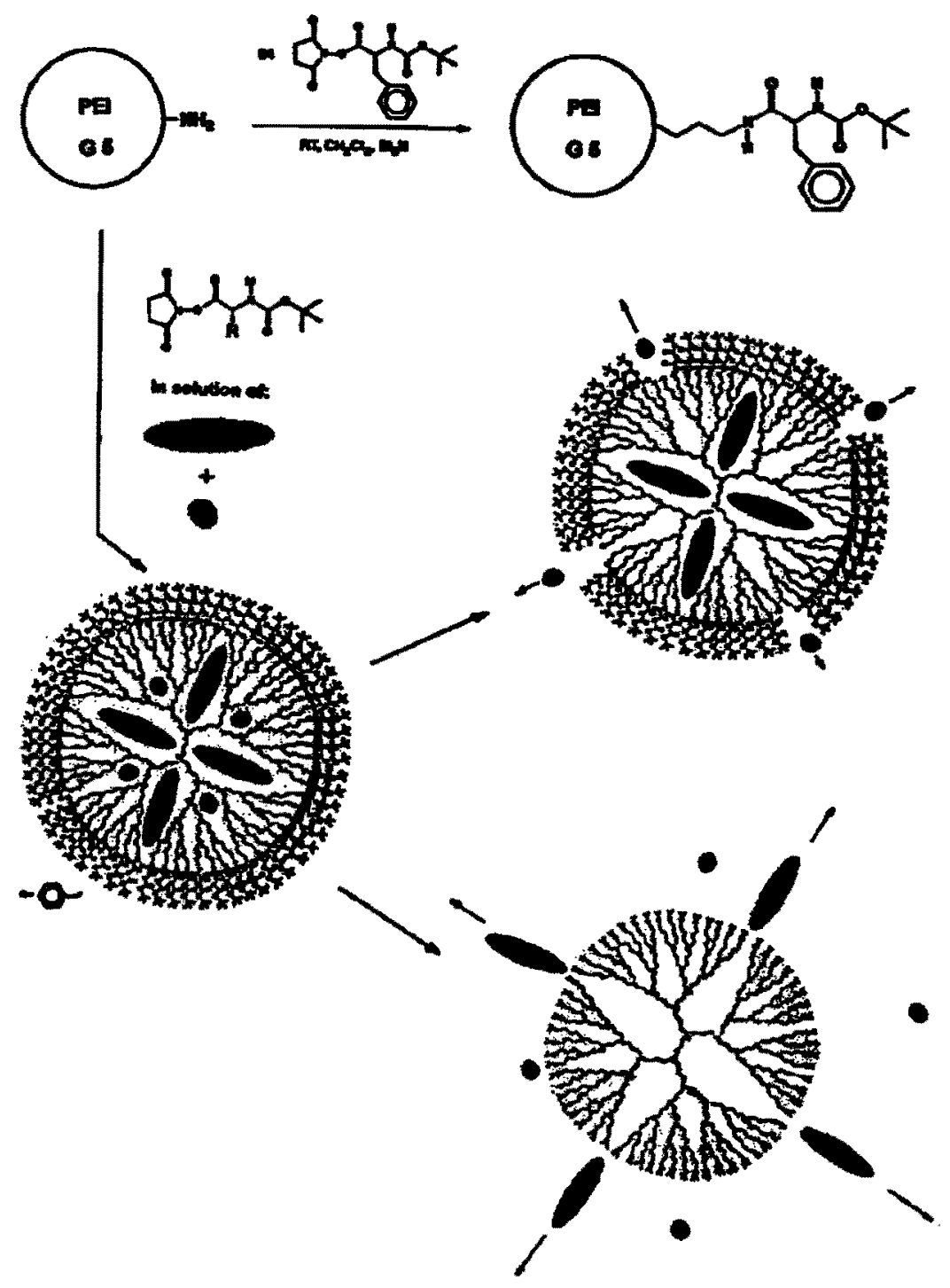

Figure 3-8. The molecular encapsulation principle: closing and opening of a dendrimer box. The starting dendrimer is a generation five (G5) amino terminated PEI with $64 \mathrm{NH} 2$ surface groups. Two different types of inert solute molecules and entrapped and selectively released. 75 


\subsection{Characterization}

The characterization of dendrimers is rather complex due to the size of and symmetry in these macromolecules. Various NMR techniques $\left({ }^{1} \mathrm{H},{ }^{13} \mathrm{C},{ }^{15} \mathrm{~N},{ }^{31} \mathrm{P}\right)$, elemental analyses, and chromatography techniques (HPLC, SEC) are widely used, but these techniques cannot reveal small amounts of impurities, particularly in higher generation dendrimers. ${ }^{76}$ Recent progress in ESI (electrospray ionization) and MALDI (matrix-assisted laser desorption ionization) mass spectrometry allows for an in-depth analysis of dendrimers. ESI-MS has been used to identify the imperfections in both poly(propylene imine) and poly amido amine (PAMAM) dendrimers. ${ }^{77-80}$

All generations of poly(propylene imine) dendrimers with either amine or nitrile end groups have been analyzed with ESI-MS to quantitatively determine the importance of various side reactions. ${ }^{81}$ The results indicated that two different pathways which account for the formation of defect structures; 1) incomplete cyanoethylations and retroMichael reactions, 2) the intramolecular amine formations (cyclizations). ESI-MS studies on PAMAM dendrimers indicate defect structures arising from retro-Michael additions and intramolecular lactam formations. ${ }^{77-80}$ MALDI-MS studies on other divergently produced higher generation dendrimers, such as Newkome's arborol dendrimers 82,83 and carbosilanes ${ }^{84-87}$ have also shown the presence of small numbers of imperfect structures. 


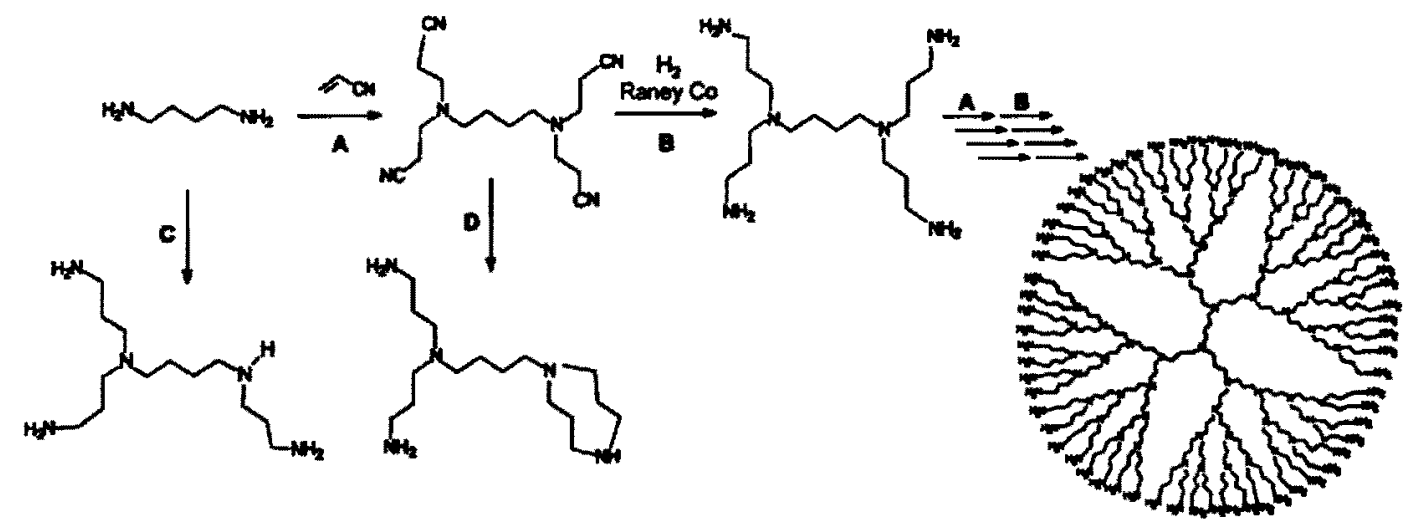

Figure 3-9. The synthesis of PPI dendrimers (reactions A and B) and alternative, unwanted reaction paths C and D. Path C illustrates "missed" Michael additions. Path D illustrates unwanted cyclization reactions. Paths $\mathrm{C}$ and $\mathrm{D}$ describe defect reactions continuing from one amine generation to the next. 81<smiles>COC(=O)/C=C/C(=O)OC</smiles>

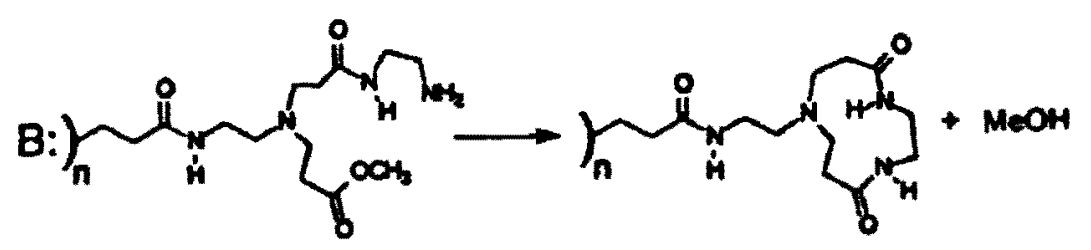

Figure 3-10. Unwanted reactions in the PAMAM-synthesis: retro-Michael reaction (A) and lactam formations (B). ${ }^{77}$ 
Dendrimers synthesized via the convergent approach can be produced nearly pure, as confirmed by MS data. The two dendrimers best representing the convergent approach are Fréchet's aromatic polyether dendrimers and Moore's phenylacetylene dendrimers. MALDI mass spectra of Fréchet-type dendrimers display very limited amounts of impurities. ${ }^{45,88}$ Almost no impurities have been found for a dendrimer with a mass of 39,969 Dalton of Moore's poly (phenylacetylene) dendrimers. ${ }^{89}$ ESI-MS data on carboxylate-terminated phenylacetylene dendrimers confirm the high degree of purity that can be attained for these dendrimers.

\subsection{Physical Behavior of Dendritic Molecules}

3.5.1 Localization of end groups in dendrimers. Dendrimers display unusual physical properties as a consequence of their unique topologies. One of the key issues required to have a better understanding seems to be elucidation of interior and exterior dendrimer morphology. Tomalia's rheological investigations showed that, in contrast to the classical linear polymers, dendrimers exhibit typical Newtonian flow patterns even at high mass (i.e. $60,000 \mathrm{amu})$. This clearly indicates an apparent lack of macromolecular entanglement. Dendrimer backfolding is considered to be the contributing factor to the deviating properties of dendrimers as compared to other linear polymers. The polyether dendrimers synthesized by Fréchet $e t$ al. have been investigated in detail to establish the possibilities for backfolding in these molecules (Figure 3-11). 


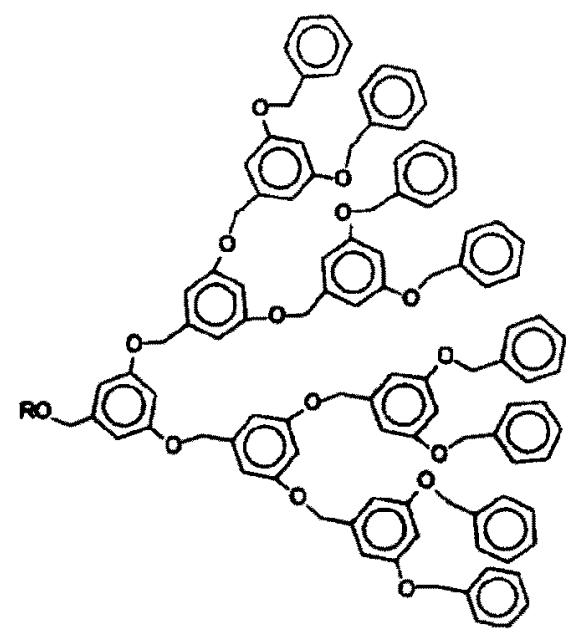

Figure 3-11. Structure of a Fréchet-type polyaryl ether dendritic wedge.

One of the first studies was done by Mourey et al. ${ }^{90}$ using size exclusion chromatography coupled to differential viscometry. They found that the hydrodynamic radii, calculated from the measured intrinsic viscosity, increase approximately linearly with dendrimer generation. A maximum in the intrinsic viscosity as a function of molecular weight was also observed. Both of these trends are in agreement with the model of Lescanec and Muthukumar, 40 implying that the end groups can be found throughout the dendrimer volume. Using the rotational-echo double-resonance (REDOR) NMR, Wooley et al. showed that backfolding also takes place in the solid state. They have found that the radial density for the fifth generation polyether dendrimer decreases monotonically with increasing distance from the center of mass. 91 In additional, Gorman et al. have measured the spin lattice relaxation (T1) in polyaryl ether dendrimers with a paramagnetic core. Their data reveal that the end groups are close to the core of the molecule. A 
recent study conducted by De Schryver et al. to determine the hydrodynamic volumes of Fréchet-type dendrimers with rubicene cores by fluorescence depolarization measurement has reported similar results to those obtained by Mourey. This supports the earlier hypothesis that the end groups are backfolded. In addition, this study has indicated that the hydrodynamic volume of the investigated dendrimers appears to be temperature independent, whereas this volume is strongly influenced by the solvent. The dendrimers are collapsed in poor solvents, while in good solvents a more open dendritic structure exists. 92

3.5.2 Dendrimers versus linear macromolecules. The intrinsic viscosity of dendrimers does not increase monotonically with molecular mass but reaches a maximum at a certain dendrimer generation. This is in contrast to linear polymers. In the solid state, the growth pattern of dendrimers determines their physical characteristics. In general, it is believed that a gradual transition in overall shape, from a more open arrangement for lower generation dendrimers to a compact and approximately globular shape for higher generation dendrimers, causes the deviation in physical behavior of dendrimers from those of linear macromolecules. The dendritic polyester solubility study conducted by Fréchet's group indicated that dendrimers are more soluble in a range of organic solvents than their linear counterparts. 93 Remarkable differences in dendrimer reactivity compared with their linear analogues were reported by two separate groups. 94,95 For all these cases, the observed differences in solubility and reactivity have been attributed to the globular architecture of the dendrimers and the accessibility of the end groups of the dendrimer. 
3.5.3 The behavior of dendrimers on surfaces. The peculiarities of dendritic structures offer new possibilities to manipulate the properties of surfaces with coatings. In preparations of dendrimers at interfaces, either gas-water or gas-solid transitions, a change in conformation compared to that in solution has been noticed. The extent of this change depends on the strength of the respective interactions. 96 The stronger the attractive forces, the more flattened the spherical shape becomes and the more it resembles a disc. Tsukruk et al. have observed the deformation of PAMAMs in monolayers on silicon surfaces. ${ }^{94,97}$ The PAMAMs are collapsed and highly compressed along the surface normal, resulting in flattened disklike structures (Figure 3-12, b). Carbosiloxane dendrimers with trimethylsilyl end groups have been visualized with scanning force microscopy (SFM). 84 On a glass substrate, single dendritic molecules are observed with globular shapes and diameters in the order of $3 \mathrm{~nm}$ (Figure 3-12, a). The materials have a strong tendency to coagulate; therefore, in addition to monomolecular structures, clusters and even droplets are also visible.
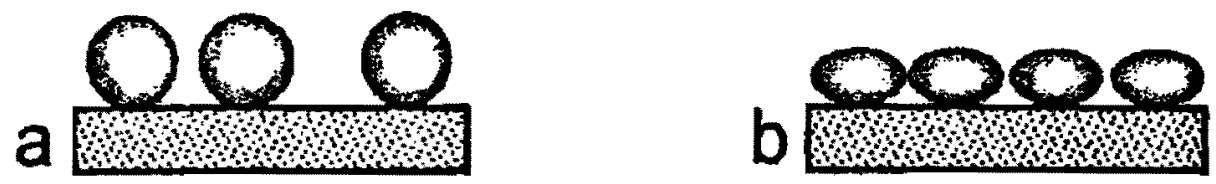

Figure 3-12. Schematic representation of the different modes of adsorption of dendrimers on surfaces: (a) adsorbed noninteracting dendrimers; 84 (b) adsorbed dendrimers with surfaceinteracting end groups. 97,98 
Interestingly, the deformation of dendrimers on surfaces has been predicted by Mansfield in a Monte Carlo study. 99 The investigation considers the adsorption of dendrimers on a surface at different interaction strengths. The calculations show a flattening of the dendrimer shape with increasing adsorption strengths. As reflected in the "phase diagram" (Figure 3-13), the mode of adsorption of the dendrimers is dependent on interaction strength and on the generation number (higher generation dendrimers have more interaction sites per molecule, and therefore, these dendrimers have stronger adsorption).

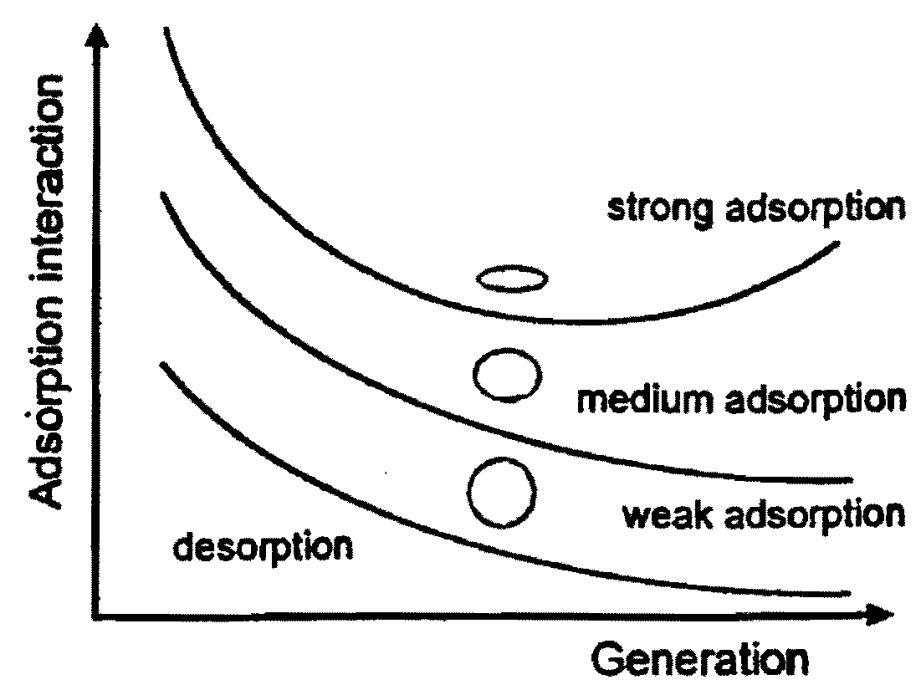

Figure 3-13. A "phase diagram" that shows the shape of dendrimers in adsorbed monolayers depending on the strength of the adsorption interaction and the dendrimer generation. The data are based on calculations by Mansfield. 99 


\subsection{Chirality}

Chiral dendrimers are another interesting feature in this field of chemistry. Several reviews covering the recent progress on the synthesis and properties of chiral dendritic molecules have been reported. ${ }^{100-102}$ Seebach et al. defined the characteristics of chiral dendrimers by the following properties: (1) their overall shape could be chiral and not spherical; (2) the arrangement of the functional groups on their surface could be chiral; (3) chiral substructures within a chiral dendrimer should be detectable by optical measurements. Figure 3-14 illustrates some of the potential dendritic structures and subclasses that can be generated. 102

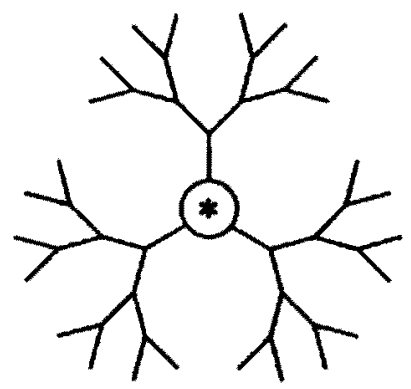

dendiner with a chiral wore (achiral or chiral branches)

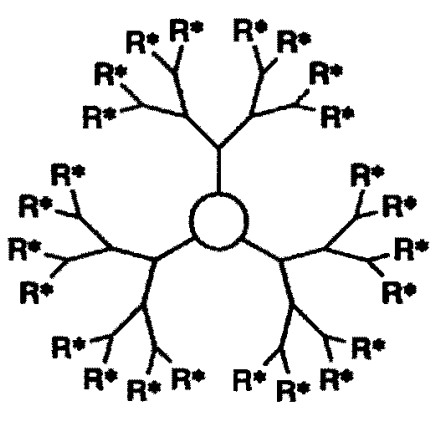

dendrimer with chiral peripheral enits (achiral or chiral corc and building blocks)

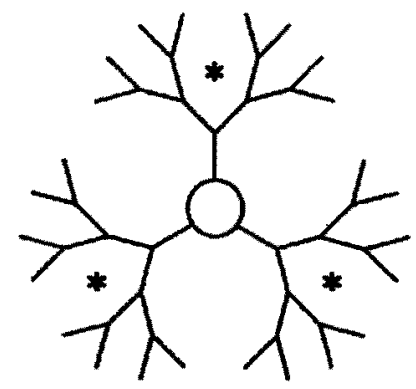

dendrimer with chiral building blocks as spacers or brankhing units (achind corr)

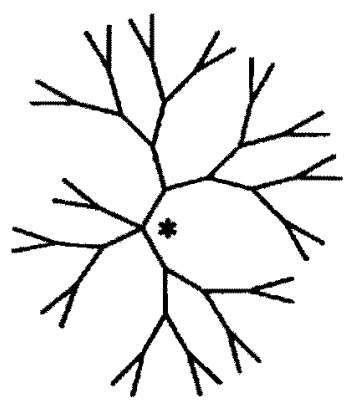

dendrimer with difforent achiral brunches attachad to non-planar core

Figure 3-14. Schematic representations of different types of chiral dendrimers. ${ }^{102}$ 
The first report of purely organic chiral dendrimers was published by Newkome et $a l .103$ They prepared a four-directional core molecule from pentaerythritol that has been elongated with tris[carboxyethoxymethyl]amino-methane branching units using standard DCC peptide coupling conditions. The desired dendrimer was then subjected to a hydrolysis to give the dodeca-acid (a). Similarly, the $2^{\text {nd }}$-generation dendrimer (b) has been obtained, which could be modified by treatment with tryptophane methyl ester to give dendrimer (c) (Figure 3-15). The molar ellipticity of these dendrimers was found to increase proportional to the number of chiral end groups.

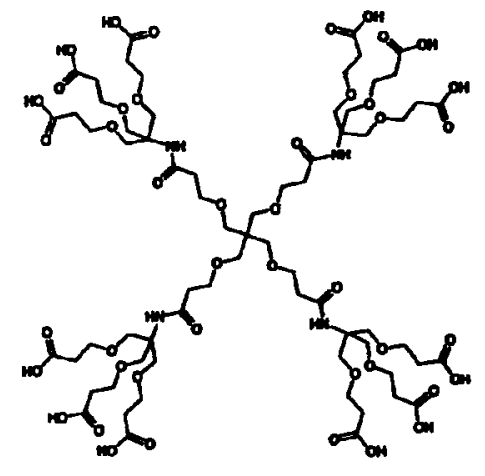

(a)

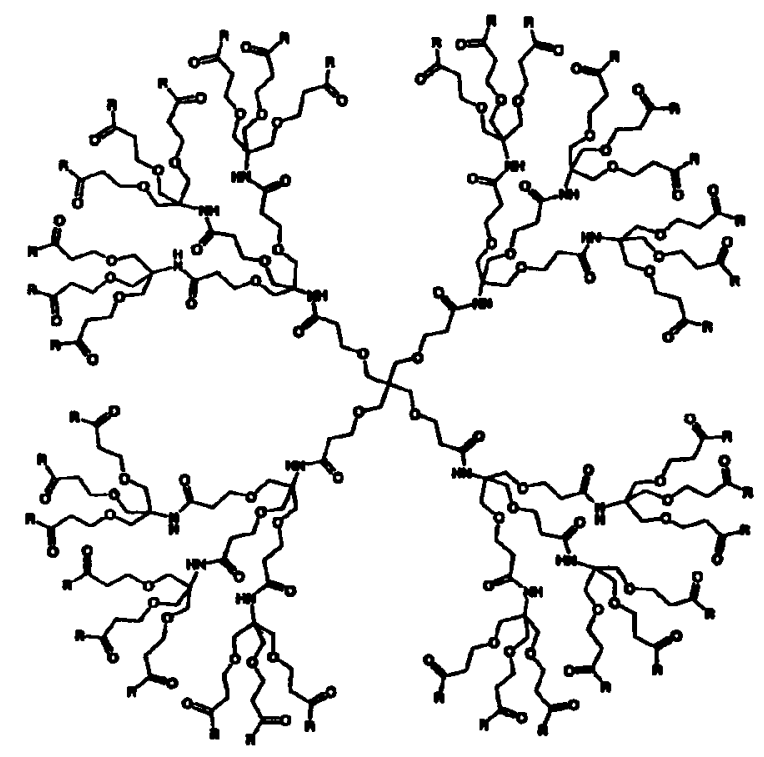

(b) $\mathrm{R}=\mathrm{OH}$

(c)

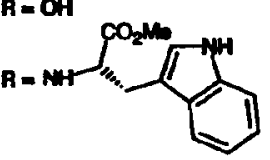

Figure 3-15. The first example of a "grafted" dendrimer prepared by Newkome $e t$ al. 103 
Seebach ${ }^{104}$ published chiral cores in dendrimers containing 12 aryl branches. The optical activity of dendrimers with chiral cores decreases with the increasing size of the dendrimer. When the dendrimer is fully chiral, the optical activity corresponds to that of the core (Figure 3-16).

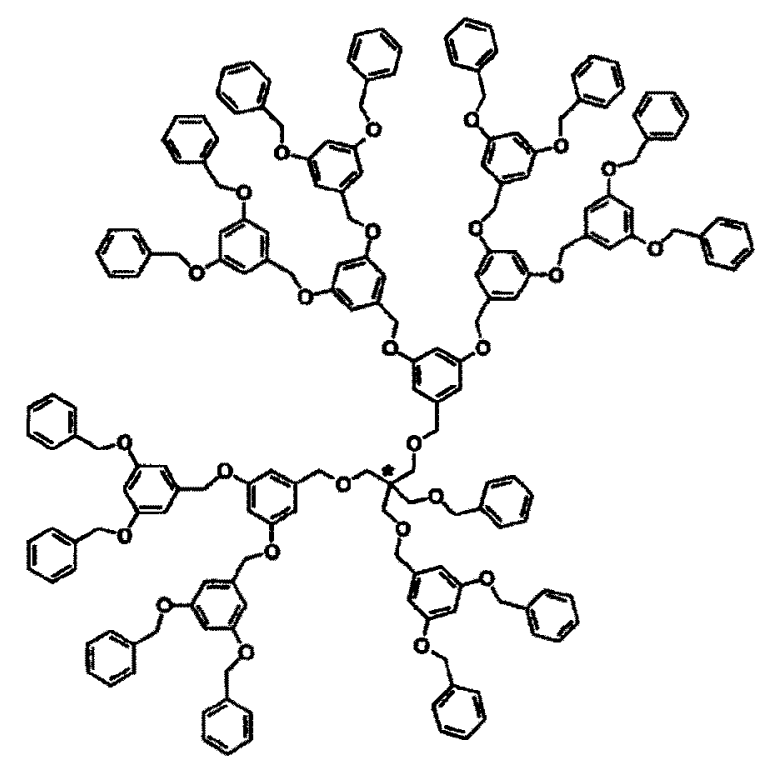

Figure 3-16. Chiral dendrimers with a core chirality center. ${ }^{105}$

Further work is required to know whether a chiral dendrimer can provide enantiomeric host-guest recognition or can have a chiral shape. The most efficient strategy for this purpose (chiral core or chiral branches or both, or a chiral core with different chiral or achiral branches) is not yet clear.

Meijer 105 has investigated the properties of dendrimers based on the chiral pentaerythrol core; he showed that chiral dendrimers with chiral terminating branches have low or vanishing optical activity. The structure (size and shape) of the cavities inside the 
dendritic molecule should be an important parameter for host-guest interactions. The simplicity afforded by their interactive synthesis allows for precise variation and functionalization and facilitates structure-property relationship studies. It is expected that other chiral dendrimers containing different stereogenic centers (e.g., biphenyls), other chiral elements (e.g., phosphorus), or larger chiral building blocks (e.g., cyclodextrins) will be synthesized and studied (Figure 3-17). Future work with chiral dendrimers will prove informative for researchers interested in stereochemistry and the manifestation of chirality in small and large molecules, and for those who are searching for new materials as well as new chiral catalysts and molecular hosts. 101
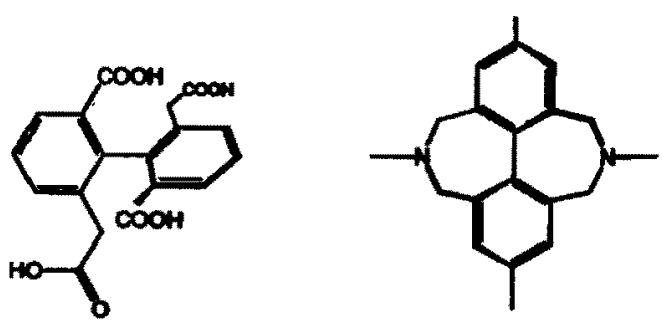

A

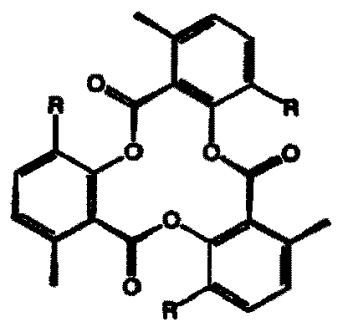

c

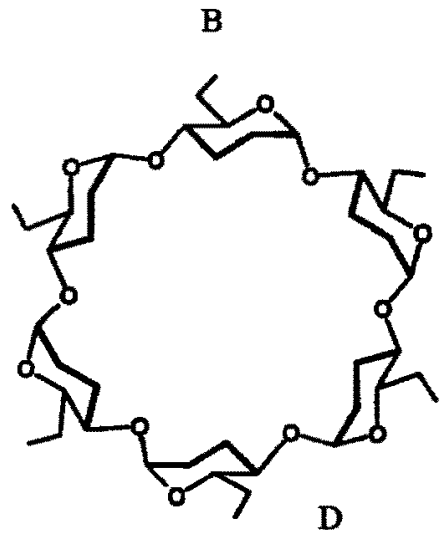

Figure 3-17. Examples of chiral cores that can potentially be utilized for the generation of chiral dendrimers: A, B: Chiral biphenyls. C: tri-othymotide derivative. D: A cyclohexaamylose ( $\alpha$-cyclodextrin) derivative. 101 


\subsection{References for Chapter 3}

1) Tomalia, D. A.; Naylor, A. M.; Goddard, W. A. Agnew. Chem. Int. Ed. Engl. $1990,29,138$.

2) Buhleier, E.; Wehner, W.; and Vogtle, F. Synthesis 1978.

3) Denkewalter, R. G.; Kolc, J. F.; Lukcasavage, W. J. U. S. Patent 4,410,688 1983.

4) Tomalia, D. A.; Baker, H.; Dewald, J.; Hall, M.; Kallos, G.; Martic, S.; Roeck, J.;

Ryder, J.; Smith, P. Polym. J. 1985, 17, 117.

5) Newkome, G. R.; Yao, Z.; Baker, G. R. a. G., V. K. J. Org. Chem. 1985, 50, $2004-2006$.

6) Alper, J., Science 1991, 251, 1562-1564.

7) Service, R. F. Science 1995, 267, 458-9.

8) Kuzdzal, S. A.; Monnig, C. A.; Newkome, G. R.; Moorefield, C. N. J. Am. Chem. Soc. 1997, 119, 2255-2261.

9) Tomalia, D. A. Sci. Am. 1995, 273, 62-66.

10) Wiener, E. C.; Brechbiel, M. W.; Brothers, H.; Magin, R. L.; Gansow, O. A.; Tomalia, D. A.; Lauterbur, P. C. Magn. Res. Med. 1994, $31,1$.

11) Zimmerman, S. C. Curr. Opin. Colloid Interface Sci. 1997, 2, 89-99.

12) Weinmann, H. J.; Ebert, W.; Misselwitz, B.; Raduchel, B.; Schmitt-Willich, H.; Platzek, J. Eur. Radiol. 1997, 7, 196.

13) Flory, P. J. J. Am. Chem. Soc. 1941, 63, 3083.

14) Flory, P. J. J. Am. Chem. Soc. 1941, 63, 3091.

15) Cram, D. J.; and Cram, J. M. Science 1974, 183, 803-809.

16) Cram, D. J. Angew. Chem. Int. Ed. Engl. 1988, 27, 1009-1020. 
17) Pedersen, C. J. Angew. Chem. Int. Ed. Engl. 1988, 27, 1021-1027.

18) Lehn, J. M. Acc. Chem. Res. 1978, 11, 49-57.

19) Lehn, J. M. Pure Appl. Chem. 1978, 50, 871-892.

20) Lehn, J. M. Science 1985, 227, 849-856.

21) Lehn, J. M. Angew. Chem. Int. Ed. Engl. 1988, 27, 89-112.

22) Suckling, C. J. J. Chem. Soc., Chem. Commun. 1982, 661-662.

23) Hyatt, J. A. J. Org. Chem. 1978, 43, 1808-1811.

24) Vogtle, F.; and Weber, E. Angew. Chem. Int. Ed. Engl. 1974, 13, 814-815.

25) Murakami, Y.; Nakano, A.; Akiyoshi, K.; and Fukuya, K. J. Chem. Soc. Perkin Trans 1974, 1, 2800-2808.

26) Tomalia, D. A.; Baker, H.; Dewald, J.; Hall, M.; Kallos, G.; Martin, S.; Roeck, J.; Ryder, J.; Smith, P. Macromolecules 1986, 19, 2466-8.

27) Tomalia, D. A.; Hall, M.; Hedstrand, D. M. J. Am. Chem. Soc. 1987, 109, 1601.

28) Tomalia, D. A.; Berry, V.; Hall, M.; and Hedstrand, D. M. Macromolecules, 1987, $20,1164-1167$.

29) Newkome, G. R.; Baker, G. R.; Saunders, M. J.; Russo, P. S.; Gupta, V. K.; Yao, Z.; Miller, J. E. a. B., K. J. Chem. Soc., Chem. Commun 1986, 752-753.

30) Newkome, G. R.; Yao, Z.; Baker, G. R.; Gupta, V. K.; Russo, P. S. a. S., M. J. J. Am. Chem. Soc. 1986, 108, 8490-850.

31) Frechet, J. M. J.; Jiang, Y.; Hawker, C. J.; Philippides, A. E. Proc. IUPAC Int. Symp. Macromol, Seoul 1989, 19.

32) Miller, T. M.; Neenan, T. X. Chem. Mater. 1990, 2, 346-9.

33) Meijer, E. W.; and Jansen, J. F. G. A. J. Am. Chem. Soc. 1995, 117, 4417-4418. 
34) Zimmerman, S. C.; Zeng, F.; Reichert, D. E. C.; Kolotuchin, S. V. Science 1996, 271, 1095-98.

35) Voegtle, F.; Newkome, G. R.; Moorefield, C.; Editors Dendritic Molecules: Concepts, Syntheses, Perspectives, 1996.

36) Aharoni, S. M.; Crosby, C. R. I.; and Walsh, E. K. Macromolecules 1982, 15, 1093.

37) De Gennes, P. G.; and Hervet, H. J. Phys Lett. 1983, 44, L351.

38) Muthukumar, M.; and Lescanec, R. L. Macromolecules 1990, 23, 2280.

39) Boris, D.; Rubinstein, M. Macromolecules 1996, 29, 7251.

40) Donners, J. J. J. M.; Heywood, B. R.; Meijer, E. W.; Nolte, R. J. M.; Sommerdijk, N. A. J. M. Book of Abstracts, 217th ACS National Meeting, Anaheim, Calif., March 21-25 1999, ORGN-086.

41) Lange, R. F. M.; Van Gurp, M.; Meijer, E. W. J. Polym. Sci., Part A: Polym. Chem. 1999, 37, 3657-3670.

42) Frechet, M. J. J. Am. Chem. Soc., 1996, 118, 3785.

43) Pollak, K. W.; Sanford, E. M.; Frechet, J. M. J. J. Mater. Chem. 1998, 8, 519-527.

44) Hawker, C. J.; Frechet, J. M. J. Macromolecules 1990, 23, 4726-9.

45) Rannard, S.; and Davis, N. In Proceedings of the American Chemical Society, Division of Polymeric Materials: Science and Engineering: 1997, Sept. 8-11; Las Vegas, Nevada. ACS; 1997, 77, 160-161.

46) Moore, J. S.; Wilkins, C. L.; Kawaguchi, T.; Walker, K. L. J. Am. Chem. Soc. $1995,117,2159-2165$. 
47) Frechet, J. M. J.; and Hawker, C. J. J. Chem. Soc. Chem. Commun. 1990, 10101013.

48) Frechet, J. M. J.; Hawker, C. J.; and Wooley, K. L. J. Am. Chem. Soc. 1991, 113, $4252-4261$.

49) Frechet, J. M. J.; Hawker, C. J.; and Wooley, K. L. Angew. Chem. Int. Ed. Engl., $1994,33,82-85$.

50) Frechet, J. M. J.; and Spindler, R. J. Chem. Soc. Perkin Trans. 1993, 1, 913-918.

51) Zimmerman, S. C.; F., a. Z. J. Am. Chem. Soc., 1996, 118, 5326-5327.

52) Bradley, M.; Swali, V.; Wells, N. J.; and Langley, G. J. J. Org. Chem. 1997, 62, 4902-4903.

53) Rebrov, E. A.; Muzafarov, A. M.; Papkov, V. S.; and Zadnov, A. A. Dokl Alad Nauk SSSR 1989, 309, 376.

54) Morikawa, A.; Kakimoto, M.; and Imai, Y. Macromolecules 1991, 24, 3469-3474.

55) Suzuki, H.; Kimata, Y.; Satoh, S.; and Kuriyama, A. Chem. Lett. 1995, 293-294.

56) Lambert, J. B.; Pflug, J. L.; and Stern, C. L. Angew. Cem. Int. Ed. Engl. 1995, 34, 98-99.

57) Sekiguchi, A.; Nanjo, M.; Kabuto, C.; and Sakurai, H. J. Am. Chem. Soc. 1995, $117,4195-4196$.

58) Majoral, J. P.; Launay, N.; and Caminade, A. M. J. Am. Chem. Soc. 1995, 117, 3282- 3283.

59) Majoral, J. P.; Galliot, C.; Prevote, D.; and Caminade, A. M. J. Am. Chem. Soc. $1995,117,5407-5476$. 
60) Slany, M.; Bardaji, M.; Casanove, M.-J.; Caminade, A.-M.; Majoral, J.-P.;

Chaudret, B. J. Am. Chem. Soc. 1995, 117, 9764-5.

61) Bardaji, M.; Kustos, M.; Caminade, A.-M.; Majoral, J.-P.; Chaudret, B.

Organometallics 1997, 16, 403-410.

62) Lartige, M. L.; Slany, M.; Caminade, A. M.; and Majoral, J. P. Chem. Eur. J. $1997,2,1417-1426$.

63) Campagna, S.; Denti, G.; Serroni, S.; Juris, A.; Venturi, M.; Ricevuto, V.; Balzani, V. Chem. Eur. J. 1995, 1.

64) Xu, Z.; Moore, J. S. Acta Polym. 1994, 45, 83.

65) Hobson, L. J.; Harrison, R. M. Curr. Opin. Solid State Mater. Sci., 1997, 2(6), 683.

66) Liao, Y.-H.; Moss, J. R. Organometallics 1996, 15, 4307.

67) Cuadrado, I.; Moran, M.; Casado, C. M.; Alonso, B.; Lobete, F.; Garacia, B.; Ibisate, M.; Losada, J. Organometallics 1996, 15, 5278.

68) Bosman, A. W.; Schenning, A. P. H. J.; Jassen, R. A. J.; Meijer, E. W. Chem. Ber. $1997,130,725$.

69) Tzalis, D.; Tor, Y. Tett. Lett. 1996, 37, 8293.

70) Zimmerman, S. C.; Zeng, F.; Reichert, D. E. C.; Kolotuchin, S. V. Science 1996, $271,1095$.

71) Thiyagarajan, P.; Zeng, F.; Ku, C. Y.; Zimmerman, S. C. J. Mater. Chem. 1997, 7, 1221.

72) Jansen, J. F. G. A.; de Brabander van den Berg, E. M. M.; Meijer, E. W. Science $1994,266,1226-9$. 
73) Jansen, J. F. G. A.; Meijer, E. W.; de Brabander-van den Berg, E. M. M. J. Am. Chem. Soc. 1995, 117, 4417-18.

74) Pesak, D. J.; Moore, J. D.; Wheat, T. E. Macromolecules 1997, 30.

75) Tomalia, D. A.; Kallos, G. J.; Hedstrand, D. M.; Lewis, S.; Zhou, J. Rapid Commun. Mass Spectrom. 1991, 5.

76) Schwartz, B. L.; Rockwood, A. L.; Smith, R. D.; Tomalia, D. A.; Spindler, R. Rapid Commun. Mass Spectrom. 1995, 9, 1552-5.

77) Dvornic, P. R.; Tomalia, D. A. Macromol. Symp. 1995, 98, 403-28.

78) Tolic, L. P.; Anderson, G. A.; Smith, R. D.; Brothers, H. M., II; Spindler, R.; Tomalia, D. A. Int. J. Mass Spectrom. Ion Processes 1997, 165, 405-418.

79) Hummelen, J. C.; Van Dongen, J. L. J.; Meijer, E. W. Chem.--Eur. J. 1997, 3, 1489-1493.

80) Dandliker, P. J.; Diederich, F.; Gross, M.; Knobler, C. B.; Loutai, A.; Sanford, E. M. Angew. Chem. Int. Ed. Engl. 1994, 33, 1739-1742.

81) Dandliker, P. J.; Diederich, f.; Gisselbrecht, J. P. C. B.; Louati, A.; Gross, M. Angew. Chem. Int. Ed. Engl. 1995, 34, 2725-2728.

82) Lorenz, K.; Mulhaupt, R.; Frey, H.; Rapp, U.; Mayer-Posner, F. J. Macromolecules 1995, 28, 6657-6661.

83) Sheiko, S. S.; Eckert, G.; Ignat'eva, G.; Muzafarov, A. M.; Spikermann, J.; Rader, H. J.; Moller, M. Macromol. Rapid Commun. 1996, 17, 283-297.

84) Wu, Z.; Biemann, K. Int. J. Mass Spectrom. Ion Proc, 1997, 165, 349-261.

85) Krska, S. W.; Seyferth, D. J. Am. Chem. Soc. 1998, 120, 3604-3612.

86) Leon, J. W.; Frechet, J. M. J. Polym. Bull. 1995, 35, 449-455. 
87) Kawaguchi, T.; Walker, K. L.; Wilkins, C. L.; Moore, J. S. J. Am. Chem. Soc. $1995,117,2159-2165$.

88) Mourey, T. H.; Turner, S. R.; Rubinstein, M.; Frechet, J. M. J.; Hawker, C. J.;

Wooley, K. L. Macromolecules 1992, 25, 2401-6.

89) Wooley, K. L.; Klug, C. A.; Tasaki, K; Schaefer, J. J. Am. Chem. Soc., 1997, 119, 53.

90) De Backer, S.; Prinzie, Y.; Verheijen, W.; Smet, M.; Desmedt, K.; Dehaen, W.; De Schryver, F. C. J. Phys. Chem. A 1998, 102, 5451.

91) Wooley, K. L.; Frechet, J. M. J.; Hawker, C. J. Polymer 1994, 35, 4489.

92) Bosman, A. W.; Janssen, H. M.; Meijer, E. W. Chem. Rev. (Washington, D. C.) $1999,99,1665-1688$.

93) de Brabander, E. M. M.; Brackman, J.; Mure-Mak, M.; de Man, H.; Hogeweg, M.; Keulen, J.; Scherrenberg, R.; Coussens, B.; Mengerink, Y.; van der Wal, S. Macromol. Symp. 1996, 102, 9.

94) Frey, H.; Lach, C.; Lorenz, K. Adv. Mater. 1998, 10, 279.

95) Tsukruk, V. T. Adv. Mater. 1998, 10, 253.

96) Bliznyuk, V. N.; Rinderspacher, F.; Tsukruk, V. V. Polymer 1998, 39, 5249.

97) Mansfield, M. L. Polymer 1996, 37, 3835.

98) Chow, H.-F.; Mong, T. K. K.; Wan, C.-W.; Wang, Z. Y. Adv. Dendritic Macromol. 1999, 4, 107-133.

99) Thomas, C. W.; Tor, Y. Chirality 1998, 10, 53-59.

100) Seebach, D.; Rheiner, P. B.; Greiveldinger, G.; Butz, T.; Sellner, H. Top. Curr. Chem. 1998, 197, 125-164. 
101) Newkome, G. R.; Lin, X.; Weis, C. D. Tetrahedron: Asymmetry 1991, 2, 957-60.

102) Seebach, D.; Lapierre, J.-M.; Skobridis, K.; Greiveldinger, G. Agnew. Chem. Int. Ed. Engl. 1994, 33, 440.

103) Kremers, J. A.; Meijer, E. W. J. Org. Chem. 1994, 59, 4262-6. 


\section{CHAPTER 4}

\section{Dendrimer Synthesis}

\subsection{Introduction}

The synthesis of dendritic polymers has developed along two archetypes: the divergent and convergent approaches. Examples of both methodologies were given in an earlier chapter. The convergent synthesis of the poly(aryl ether) dendrimers described in this dissertation was based mainly on the early work of Fréchet. ${ }^{1}$ However, the coupling of the lower generation bromides with the 3,5-dihydroxy benzyl alcohol monomer involved the use of triphenylphosphine. The purification of higher generation dendrimers was difficult because triphenylphosphine was not easily separated from the desired product. In this research, the author developed a different reaction scheme to successfully couple the lower generation dendrons with the monomer. A mesylate intermediate was developed which then coupled to the monomer. A schematic reaction diagram is shown in Figure 4-1 to illustrate the modified synthesis route. Dendrimers generated from this process were separated using preparative LC chromatography. 
<smiles>O=C(O)C1CC(O)CC(O)C1</smiles><smiles>COS(=O)(=O)O</smiles><smiles>CC(=O)c1cc(O)ccc1O</smiles><smiles>O=C1CC2CCC(O2)C1OCc1ccccc1</smiles>

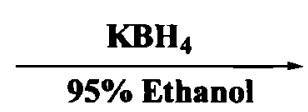<smiles>OC[C@H]1CC(OCC2CCCCC2)CC1OCC1CCCCC1</smiles><smiles>OCC1CC(OCC2=CCCC2)CC(OCc2ccccc2)C1</smiles>

(2)

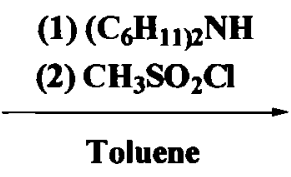

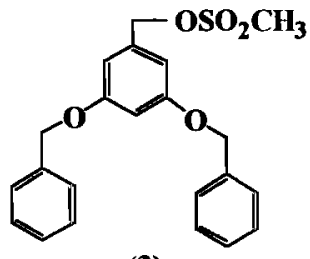

(3)

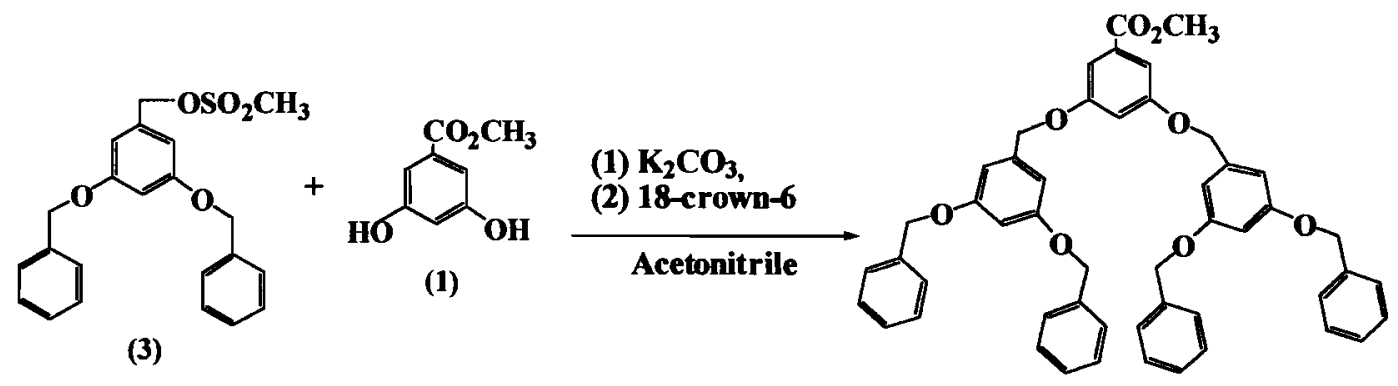

(4)

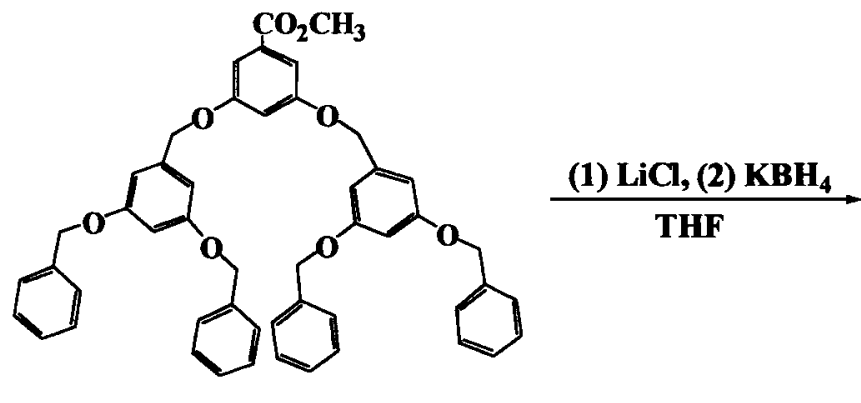

(4)

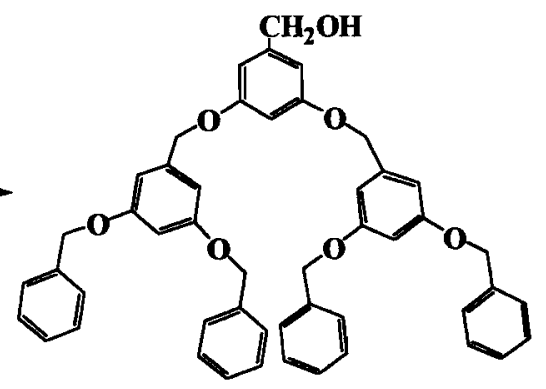

(5)

Figure 4-1. Synthetic scheme illustrating the mesylation route. 


\subsection{Experimental}

4.2.1 Materials: All solvents purchased from commercial vendors were used without further purification except for tetrahydrofuran (THF). THF was purified by distillation from sodium/benzophenone before use to give anhydrous, inhibitor-free THF. Anhydrous toluene was purchased from Aldrich Chemical Company. HPLC grade methanol and acetonitrile from Burdick-Jackson chemical company were used in all high performance liquid chromatography (HPLC) experiments. The following compounds: 3 , 5-dihydroxybenzyl alcohol; 3, 5-dihydroxybenzoic acid; 3,5-dibenzyloxybenzaldehyde; 3, 5-dimethoxybenzyl alcohol; benzyl alcohol; 4-pyridyl carbinol; N, N, N' trimethyl ethylene diamine; 3-(triethoxysilyl)propyl isocyanate; 18-crown-6; lithium chloride; potassium borohydride; carbon tetrabromide; triphenyl phosphine; methane sulphonyl chloride; 4-bromobenzene sulphonyl chloride; triethylamine; potassium carbonate; diisopropyl ethyl amine; pyridine; dicyclohexylamine; 4-(dimethylamino)pyridine; and potassium hydroxide are commercially available and were used as received from Aldrich Chemical Company.

4.2.2 Chromatography. Thin layer chromatography (TLC) on silica gel 60 F254 type E plates from EM Science and reverse phase (RP) plates from Whatman were used to monitor the completion of each reaction. Reverse phase high performance liquid chromatography (RP-HPLC) was used to optimize reaction conditions and to verify the purity of the products. All HPLC experiments were performed on a Hewlett Packard liquid chromatography instrument, model 1090 . The columns used to monitor the reactions were Luna $\mathrm{C} 18,25 \mathrm{~cm}$ x $4.6 \mathrm{~mm} \mathrm{ID,} 5 \mu$ by Phenomenex; Supelco ABZ, $7.5 \mathrm{~cm} \times$ 
4.6 mm ID, $3 \mu$ by Supelco; MICRA NPS (non-porous silica) RP18, $1.5 \mu$, by Micra Scientific Company and a preparative column, LUNA $5 \mu, \mathrm{C18}(2), 25 \mathrm{~cm} \times 10 \mathrm{~mm}$ by Phenomenex.

4.2.3 Instrumentation. All materials synthesized were characterized and identified using nuclear magnetic resonance (NMR), fourier transform infrared (FTIR), and mass spectroscopy coupled with liquid chromatography (LC/MS). The most appropriate technique was used to elucidate the molecular structure of the synthesized compounds.

'H-NMR spectra were acquired on a Bruker 300, MHz, FT-NMR. Tetramethylsilane (TMS) was used as an internal reference and data is reported in $\delta$ units. In the ${ }^{~} \mathrm{H}-\mathrm{NMR}$ data: Ar refers to protons on the aromatic ring and the subscript refers to the generation of the ring, where generation 0 is benzyl bromide (either focal point or terminus). FTIR and Raman spectra were acquired on a Perkin Elmer model 2000 using suitable sampling techniques. Mass spectra were performed using either direct loop injection or coupled with HPLC to obtain the protonated molecular ion, $[\mathrm{M}+\mathrm{H}]^{+}$. Electronic absorption spectra were obtained on a Perkin Elmer photodiode array UV-Vis spectrophotometer Lambda 14 with UV winlab software. Atomic force microscopy images were scanned on a Park Scientific Instrument, model AutoProbe CP. Melting points were determined on a Mel-temp 50/60, 110/120 volts 200 watts apparatus. Elemental analyses were performed by Quantitative Technologies Inc, Whitehouse, NJ, where a Perkin Elmer automated CHN elemental analyzer, model 2400 was used. 


\subsection{Synthesis of Dendritic Polymers}

Synthetic details for the structures shown in Figure 4-2 are provided below.<smiles>CC(=O)c1cc(O)cc(O)c1</smiles><smiles>OCc1cc(OCc2ccccc2)cc(OCc2ccccc2)c1</smiles><smiles></smiles>

(4)

(2)<smiles>COOc1cc(OCc2ccccc2)cc(OCc2ccccc2)c1</smiles>

(3)

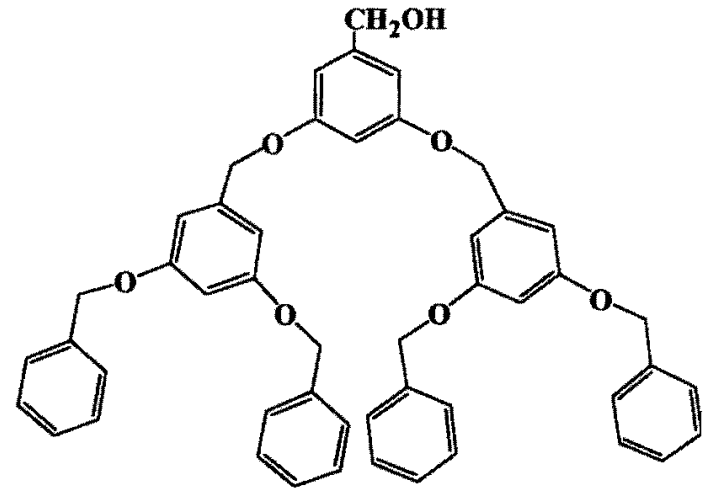

(5)

Figure 4-2. Chemical structures of the poly(aryl ether) dendrimers and the process intermediates. 
4.3.1 Methyl 3, 5-dihydroxybenzoate (1). ${ }^{2,3} \mathrm{C}_{8} \mathrm{H}_{8} \mathrm{O}_{4}$, Mol. Wt. 168.148. A solution of $154 \mathrm{~g} \mathrm{(1} \mathrm{mol)} \mathrm{of} \mathrm{3,} \mathrm{5-dihydroxybenzoic} \mathrm{acid} \mathrm{in} 400 \mathrm{~mL}$ of methanol was stirred under Nitrogen $\left(\mathrm{N}_{2}\right)$. The reaction flask was then placed in an ice bath and cooled to $0^{\circ} \mathrm{C}$. Sulfuric acid $(40 \mathrm{~mL}, 0.75 \mathrm{~mol})$ was then added slowly by an addition funnel to the cooled reaction mixture. (Caution! Strong exothermic reaction.) When the acid addition was completed, the solution was heated to reflux for 1 hour. The mixture was allowed to cool and an equal volume of distilled deionized water $\left(\mathrm{dd}_{2} \mathrm{O}\right)$ was added to quench the reaction. The aqueous alcohol mixture was extracted with diethyl ether using twice the volume of the mixture. The mixture was extracted two more times with diethyl ether using an equal volume of the mixture. The combined organic layers were washed once with $10 \%$ sodium bicarbonate $\left(\mathrm{NaHCO}_{3}\right)$ solution and once with saturated sodium chloride $(\mathrm{NaCl})$ solution. The organic layer was dried over anhydrous magnesium sulfate $\left(\mathrm{MgSO}_{4}\right)$. The solution was filtered, and removal of the solvent in vacuo gave 1 , a solid with a slight pink color. The crude product was re-dissolved in a minimum amount of hot $\mathrm{dd}_{2} \mathrm{O}$ and the product was re-crystallized upon cooling to give $136.80 \mathrm{~g}(0.81 \mathrm{~mol}$, $84 \%$ yield) 1 as off-white crystals. MP $164^{\circ} \mathrm{C}$. FTIR ( $\mathrm{KBr}$ pellet) $3250 \mathrm{~cm}^{-1}(\mathrm{OH}, \mathrm{st})$, $1694 \mathrm{~cm}^{-1}(\mathrm{C}=\mathrm{O}, \mathrm{st}), 1600 \mathrm{~cm}^{-1}\left(\mathrm{C}=\mathrm{C}\right.$, ar ring vibration), $1487 \mathrm{~cm}^{-1}\left(\mathrm{CH}_{3}\right.$ deformation), $1340 ; 1260 \mathrm{~cm}^{-1}\left(\mathrm{C}-\mathrm{O}\right.$, d, as, st), $1000 \mathrm{~cm}^{-1}\left(\mathrm{C}-\mathrm{O}\right.$, st). ${ }^{1} \mathrm{H}-\mathrm{NMR}\left(\mathrm{CDCl}_{3}, \mathrm{TMS}\right): \delta 6.926$; $6.919\left(\mathrm{~d}, \operatorname{Ar}_{0} \mathrm{H}, \mathrm{C}_{2}, 5-\mathrm{H}, 2 \mathrm{H}\right), 6.48\left(\mathrm{~s}, \mathrm{Ar}_{0} \mathrm{H}, \mathrm{C}_{4}-\mathrm{H}, 1 \mathrm{H}\right), 4.92(\mathrm{~s}, \mathrm{O}-\mathrm{H}, 2 \mathrm{H})$, and 3.85 (s, $\left.\mathrm{CH}_{3}, 3 \mathrm{H}\right)$. ESI mass spectrum: $[\mathrm{M}+\mathrm{H}]^{+}, 169$; sodium adduct ion $[\mathrm{M}+\mathrm{Na}]^{+}, 191$; and $\mathrm{m} / \mathrm{z}, 137$ fragment due to loss of $\mathrm{CH}_{3} \mathrm{OH}$ (169-32). Elemental analysis calculated for $\mathrm{C}_{8} \mathrm{H}_{8} \mathrm{O}_{4}: \mathrm{C}, 57.14 ; \mathrm{H}, 4.80$, found: $\mathrm{C}, 57.06 ; \mathrm{H}, 4.69$. 
4.3.2 3, 5-dibenzyloxybenzylalcohol (2). ${ }^{4} \mathrm{C}_{21} \mathrm{H}_{20} \mathrm{O}_{3}$, Mol. Wt. 320.39. A solution of $31.8 \mathrm{~g}(0.10 \mathrm{~mol}, 1.0$ eq.) of 3,5 dibenzyloxybenzaldehyde in $100 \mathrm{~mL}$ of $95 \%$ ethanol was treated with $2.69 \mathrm{~g}\left(0.05 \mathrm{~mol}, 0.5 \mathrm{eq}\right.$.) of potassium borohydride $\left(\mathrm{KBH}_{4}\right)$ and stirred under $\mathrm{N}_{2}$. The mixture was stirred vigorously and heated to reflux for 3 hours. The reaction is complete when a clear pale yellow to amber colored solution is obtained. (The shade of the color depends on the color of the starting material). The solution was allowed to cool and an equal volume of $0.5 \mathrm{~N} \mathrm{HCl}$ was added to quench the reaction and to remove the excess $\mathrm{KBH}_{4}$. A white precipitate was observed. A volume of methylene chloride equivalent to twice the volume of the reaction mixture was added to the aqueous alcohol to extract the product. The aqueous layer was extracted two more times with an equal volume of methylene chloride. The combined organic layers were washed once with a saturated $(\sim 40 \%)$ ammonium chloride $\left(\mathrm{NH}_{4} \mathrm{Cl}\right)$ solution and once with a saturated $\mathrm{NaCl}$ solution, and then dried over anhydrous $\mathrm{MgSO}_{4}$. The solution was filtered, and removal of the solvent in vacuo gave product 2 as a white solid. The crude product was re-dissolved in a minimum amount of hot methylene chloride. Hexane was added to recrystallize 2 from the mixture. Upon cooling, white crystals were collected to give $28.97 \mathrm{~g}$ (0.09 mol, $94 \%$ yield). MP $79.8^{\circ} \mathrm{C}$. FTIR ( $\mathrm{KBr}$ pellet): $3263 \mathrm{~cm}^{-1}(\mathrm{OH}, \mathrm{st})$, $3065 \mathrm{~cm}^{-1}$ (Ar C-H, st), $1593 \mathrm{~cm}^{-1}\left(\mathrm{C}=\mathrm{C}\right.$, ar ring vibration), $1160 \mathrm{~cm}^{-1}(\mathrm{C}-\mathrm{O}$, as stretch for ether), $1024 \mathrm{~cm}^{-1}$ (C-O, st alcohol). The $749 \mathrm{~cm}^{-1}$ and $694 \mathrm{~cm}^{-1}$ combination bands are diagnostic for a monosubstituted phenyl (5 adjacent $\mathrm{H}$ atoms). ${ }^{1} \mathrm{H}-\mathrm{NMR}\left(\mathrm{CDCl}_{3}\right.$, TMS): $\delta 7.366\left(\mathrm{~m}, \mathrm{Ar}_{1} \mathrm{H}, 10 \mathrm{H}\right), 6.608 ; 6.601\left(\mathrm{~d}, \mathrm{Ar}_{0} \mathrm{H}, \mathrm{C}_{2,6}-\mathrm{H}, 2 \mathrm{H}\right), 6.542 ; 6.535 ; 6.528$ (t, $\left.\mathrm{Ar}_{0} \mathrm{H}, \mathrm{C}_{4}-\mathrm{H}, 1 \mathrm{H}\right), 5.010\left(\mathrm{~s}, \mathrm{Ar}_{1} \mathrm{H},-\mathrm{CH}_{2}-\mathrm{O}, 4 \mathrm{H}\right), 4.598 ; 4.582\left(\mathrm{~d}, \mathrm{Ar}_{0}-\underline{\mathrm{C}}_{2}, 2 \mathrm{H}\right)$ and $1.859 ; 1.841 ; 1.823(\mathrm{t}, \mathrm{O}-\mathrm{H}, 1 \mathrm{H})$. ESI mass spectrum: $[\mathrm{M}+\mathrm{H}]^{+}, 321$; sodium adduct ion 
$[\mathrm{M}+\mathrm{Na}]^{+}$, 343. Elemental analysis: calculated for $\mathrm{C}_{21} \mathrm{H}_{20} \mathrm{O}_{3}: 78.73 ; \mathrm{H}, 6.29$. found: $\mathrm{C}$, 78.72; $\mathrm{H}, 6.22 . \mathrm{TLC}: \mathrm{Rf}=0.24$ for product $2, \mathrm{Rf}=0.55$ for the starting material, $(70: 30)$ hexane: ethyl acetate. One spot was observed at $\mathrm{Rf}=0.24$.

\subsubsection{3, 5-dibenzyloxy benzyl methane sulphonate (3). ${ }^{5-8} \mathrm{C}_{22} \mathrm{H}_{22} \mathrm{O}_{5} \mathrm{~S}$, Mol. Wt. 398 .} A solution of $3.294 \mathrm{~g}$ (10.29 mmol, $1.0 \mathrm{eq}$.) of $2, \mathrm{G} 1$ alcohol, in $100 \mathrm{~mL}$ of anhydrous toluene was stirred under $\mathrm{N}_{2}$. The reaction flask was then cooled to $0^{\circ} \mathrm{C}$ in an ice bath. Dicyclohexylamine $(2.26 \mathrm{~mL}, 11.36 \mathrm{mmol}, 1.10 \mathrm{eq}$.$) was added slowly to the cold$ mixture through a $5 \mathrm{~mL}$ glass syringe. While maintaining the solution below $8{ }^{\circ} \mathrm{C}$, methane sulphonyl chloride ( $1.60 \mathrm{~mL}, 20.77 \mathrm{mmol}, 2.02 \mathrm{eq}$.) was added dropwise to the mixture from a $5 \mathrm{~mL}$ glass syringe. (It is extremely important to maintain the temperature below $8{ }^{\circ} \mathrm{C}$ for higher purity and higher yield.) When the addition was complete, the solution was allowed to stir for 1 hour. Upon completion of the reaction, methylene chloride $(200 \mathrm{~mL})$ was added to the mixture, and the mixture was filtered. The precipitate was washed with methylene chloride. The collected filtrate was then washed with $100 \mathrm{~mL}$ dd $\mathrm{H}_{2} \mathrm{O}$ and organic layer was collected. The aqueous layer was further extracted twice with methylene chloride $(2 \times 100 \mathrm{~mL})$. The combined organic layers were washed with $0.5 \mathrm{~N} \mathrm{HCl}(100 \mathrm{~mL}), 5 \% \mathrm{NaHCO}_{3}(100 \mathrm{~mL})$, and saturated $\mathrm{NaCl}$ solution, and dried over $\mathrm{MgSO}_{4}$. The solution was filtered, and removal of the solvent in vacuo gave $4.01 \mathrm{~g}$ (10.08 mmol, $98 \%$ yield) of 3 as pale yellow crystals when cooled under refrigeration. The highly reactive product was used immediately without further purification. If necessary, it may be stored overnight under refrigeration. FTIR $(\mathrm{KBr}$ pellet): $3065 \mathrm{~cm}^{-1}$ (Ar C-H, st), $1454 \mathrm{~cm}^{-1}\left(\mathrm{CH}_{3}\right.$, as st), $1379 \mathrm{~cm}^{-1}\left(\mathrm{SO}_{2}\right.$, st, anti-sy with

overlap of $\left.\mathrm{CH}_{3}\right), 1275 \mathrm{~cm}^{-1}\left(\mathrm{C}-\mathrm{O}-\mathrm{C}\right.$, as st), $1171 \mathrm{~cm}^{-1}\left(\mathrm{SO}_{2}\right.$, sy st). The combination 
bands of $755 \mathrm{~cm}^{-1}$ and $703 \mathrm{~cm}^{-1}$ are diagnostic for a monosubstituted aromatic ring, out of plane bending vibration. ${ }^{~} \mathrm{H}-\mathrm{NMR}\left(\mathrm{CDCl}_{3}, \mathrm{TMS}\right): \delta 7.41\left(\mathrm{~m}, \mathrm{Ar}_{1} \mathrm{H}, 10 \mathrm{H}\right), 7.25\left(\mathrm{~m}, \mathrm{Ar}_{0} \mathrm{H}\right.$, $\left.\mathrm{C}_{4}-\mathrm{H}, 1 \mathrm{H}\right), 6.634\left(\mathrm{~s}, \mathrm{Ar}_{0} \mathrm{H}, \mathrm{C}_{2,5}-\mathrm{H}, 2 \mathrm{H}\right), 5.148\left(\mathrm{~s}, \mathrm{CH}_{2}-\mathrm{SO}_{3}-\mathrm{CH}_{3}, 2 \mathrm{H}\right), 5.043\left(\mathrm{~s}, \mathrm{Ar}_{1}\right.$ $\left.-\underline{\mathrm{C}}_{2}-\mathrm{O}, 4 \mathrm{H}\right)$ and $2.83\left(\mathrm{~s},-\mathrm{SO}_{3}-\mathrm{CH}_{3}, 3 \mathrm{H}\right)$. ESI mass spectrum: $[\mathrm{M}+\mathrm{H}]^{+}, 399$; sodium adduct ion $[\mathrm{M}+\mathrm{Na}]^{+}, 421$.

4.3.4 Methyl 3, 5-(3', 5'-benzyloxybenzyloxy) benzoate (4). $\mathrm{C}_{50} \mathrm{H}_{44} \mathrm{O}_{8}$, Mol. Wt. 772. A solution of $4.01 \mathrm{~g}(10.08 \mathrm{mmol}, 1.0 \mathrm{eq})$ of 3 in $100 \mathrm{~mL}$ of acetonitrile was stirred under $\mathrm{N}_{2}$. Potassium carbonate $\left(\mathrm{K}_{2} \mathrm{CO}_{3}\right), 1.729 \mathrm{~g}$ (12.50 mmol, $1.24 \mathrm{eq}$ ), 18-crown-6, $0.25 \mathrm{~g},(0.95 \mathrm{mmol}, 0.2$ eq.) and sold (1), methyl 3, 5-dihydroxybenzoate, $0.84 \mathrm{~g}$, ( $5 \mathrm{mmol}, 0.5$ eq.) were added slowly and the solution was stirred and gently refluxed for 55 hours. Methylene chloride $(100 \mathrm{~mL})$ and $0.5 \mathrm{~N} \mathrm{HCl}(100 \mathrm{~mL})$ were added to the mixture to remove the excess $\mathrm{K}_{2} \mathrm{CO}_{3}$. The aqueous layer was extracted again twice with an equal volume methylene chloride. The combined organic layers were washed once with a saturated $\mathrm{NaCl}$ solution, then dried over anhydrous $\mathrm{MgSO}_{4}$. The solution was filtered, and removal of the solvent in vacuo gave $3.7 \mathrm{~g}$ product as a white solid. The crude product was purified by preparative liquid chromatography (Prep-LC) to give 4 . The yield was $68 \%$. MP $133-134^{\circ} \mathrm{C}$. FTIR ( $\mathrm{KBr}$ pellet): $3031 \mathrm{~cm}^{-1}$ (Ar C-H, st), $2912 \mathrm{~cm}^{-1}$ (C-H aliphatic, as st), $2873 \mathrm{~cm}^{-1}\left(\mathrm{C}-\mathrm{H}\right.$ aliphatic, sy st), $1713 \mathrm{~cm}^{-1}(\mathrm{C}=\mathrm{O}$, st $)$, $1241 \mathrm{~cm}^{-1}\left(\mathrm{C}-\mathrm{O}-\mathrm{C}\right.$, as st), $1164 \mathrm{~cm}^{-1}(\mathrm{C}-\mathrm{O}-\mathrm{C}$, sy st). The combination bands of $835 \mathrm{~cm}^{-1}$ and $725 \mathrm{~cm}^{-1}$ are characteristic of 1,3,5-trisubstituted benzene (C-H out of plane bending vibration). ${ }^{1} \mathrm{H}-\mathrm{NMR}\left(\mathrm{CDCl}_{3}, \mathrm{TMS}\right)$ : $\delta$ 7.399(m, $\mathrm{Ar}_{2}-\mathrm{H}, \mathrm{Ar}_{1}-\mathrm{H}, \mathrm{Ar}_{0}-\mathrm{H}$, 22H), $6.765\left(\mathrm{t}, \mathrm{Ar}_{1}-\mathrm{H}, \mathrm{Ar}_{0}-\mathrm{H}, 7 \mathrm{H}\right), 5.034\left(\mathrm{~s}, \mathrm{Ar}_{2}-\mathrm{C}_{2}-\mathrm{O}, 8 \mathrm{H}\right), 5.004\left(\mathrm{~s}, \mathrm{Ar}_{1}-\mathrm{CH}_{2}-\mathrm{O}-\right.$, 4H), 3.907 (s, COO- $\left.\mathrm{CH}_{3}, 3 \mathrm{H}\right)$. ESI mass spectrum: $[\mathrm{M}+\mathrm{H}]^{+}, 773 ; \mathrm{m} / \mathrm{z}=303$, the 
3, 5-dibenzyloxybenzylic fragment ion. The negative ion mass spectrum exhibited an intense $\mathrm{m} / \mathrm{z}$ ion resulting from the loss of 303 (3, 5-dibenzyloxybenzylic fragment). Elemental analysis calculated for $\mathrm{C}_{50} \mathrm{H}_{44} \mathrm{O}_{8}: \mathrm{C}, 77.70 ; \mathrm{H}, 5.74$, found: $\mathrm{C}, 77.23 ; \mathrm{H}, 5.69$.

4.3.5 3, 5-(3', 5'-benzyloxybenzyloxy) benzyl alcohol (5). $\mathrm{C}_{49} \mathrm{H}_{44} \mathrm{O}_{7}$, Mol. Wt. 744 . A solution of $4,(38.6 \mathrm{~g}, 50.0 \mathrm{mmol}, 1.0 \mathrm{eq}$.) in $100 \mathrm{~mL}$ of dry THF was stirred under $\mathrm{N}_{2}$. Lithium chloride $(\mathrm{LiCl}), 6.36 \mathrm{~g},(15.0 \mathrm{mmol}, 3.0$ eq. $)$ and $\mathrm{KBH}_{4}, 8.10 \mathrm{~g}$, ( $15.00 \mathrm{mmol}, 3.0$ eq.) were added slowly to the mixture. The reaction was refluxed with vigorous stirring under $\mathrm{N}_{2}$ until the evolution of gas is minimized. When the reaction was complete, the mixture was poured into an equal volume of dd $\mathrm{H}_{2} \mathrm{O}$ to allow the excess borohydride to react. The mixture was extracted three times with methylene chloride, and the organic layers collected and combined. The organic layer was washed with dd $\mathrm{H}_{2} \mathrm{O}$ and saturated $\mathrm{NaCl}$, and dried over $\mathrm{MgSO}_{4}$. The solution was filtered and removal of the solvent gave 5 , as a white solid. The crude product was purified by first dissolving in a minimum amount of ethyl acetate and then precipitatng by addition of hexane, giving a white solid which was collected by filtration, yield $33.6 \mathrm{~g}, 90 \%$. MP $103-106^{\circ} \mathrm{C}$. FTIR (KBr pellet): $3450 \mathrm{~cm}^{-1}(\mathrm{OH}, \mathrm{st}), 3032 \mathrm{~cm}^{-1}$ (Ar C-H, st), $2873 \mathrm{~cm}^{-1}$ (C-H aliphatic, st), $1596 \mathrm{~cm}^{-1}\left(\mathrm{C}=\mathrm{C}\right.$, ar ring vibration), $1157 \mathrm{~cm}^{-1}(\mathrm{C}-\mathrm{O}-\mathrm{C}$, asym stretch for ether), $1054 \mathrm{~cm}^{-1}$ (C-O-C, sym st). The combination bands of $833 \mathrm{~cm}^{-1}$ and $697 \mathrm{~cm}^{-1}$ are characteristic of 1,3,5-trisubstituted benzene $(\mathrm{C}-\mathrm{H}$ out of plane bending vibration). ' $\mathrm{H}-\mathrm{NMR}\left(\mathrm{CDCl}_{3}, \mathrm{TMS}\right)$ : $\delta 7.394\left(\mathrm{~m}, \mathrm{Ar}_{2}-\mathrm{H}, 20 \mathrm{H}\right), 6.667$ (s, $\mathrm{Ar}_{1}$, 2H), $6.591\left(\mathrm{~m}, \mathrm{Ar}_{1} ; \mathrm{Ar}_{0}, 7 \mathrm{H}\right), 5.022\left(\mathrm{~s}, \mathrm{Ar}_{2}-\mathrm{CH}_{2}-\mathrm{O}-, 8 \mathrm{H}\right), 4.964\left(\mathrm{~s}, \mathrm{Ar}_{1}-\mathrm{CH}_{2}-\mathrm{O}, 4 \mathrm{H}\right)$, $4.613\left(\mathrm{~s}, \mathrm{Ar}_{0}-\mathrm{C} \underline{\mathrm{H}}-, 2 \mathrm{H}\right)$, and $1.564(\mathrm{~s}, \mathrm{O}-\mathrm{H}, 1 \mathrm{H})$. ESI mass spectrum: $[\mathrm{M}+\mathrm{H}]^{+}, 745$; 
sodium adduct ion $[\mathrm{M}+\mathrm{Na}]^{+} 767$; potassium adduct ions $[\mathrm{M}+\mathrm{K}]^{+} 783$. Elemental analysis: calculated for $\mathrm{C}_{49} \mathrm{H}_{44} \mathrm{O}_{7}: \mathrm{C}, 79.01 ; \mathrm{H}, 5.95$, found: $\mathrm{C}, 78.76 ; \mathrm{H}, 5.93$.

\subsection{Synthesis of Column Coating Materials}

Figure 4-3 shows the chemical structure of the desired poly(benzyl ether) dendrimers and other alcohol materials that were used to prepare the coating materials for CEC evaluation. The carbamates coupling reaction is shown in Figure 4-4. The chemical structures of the desired carbamates are presented in Figure 4-5.

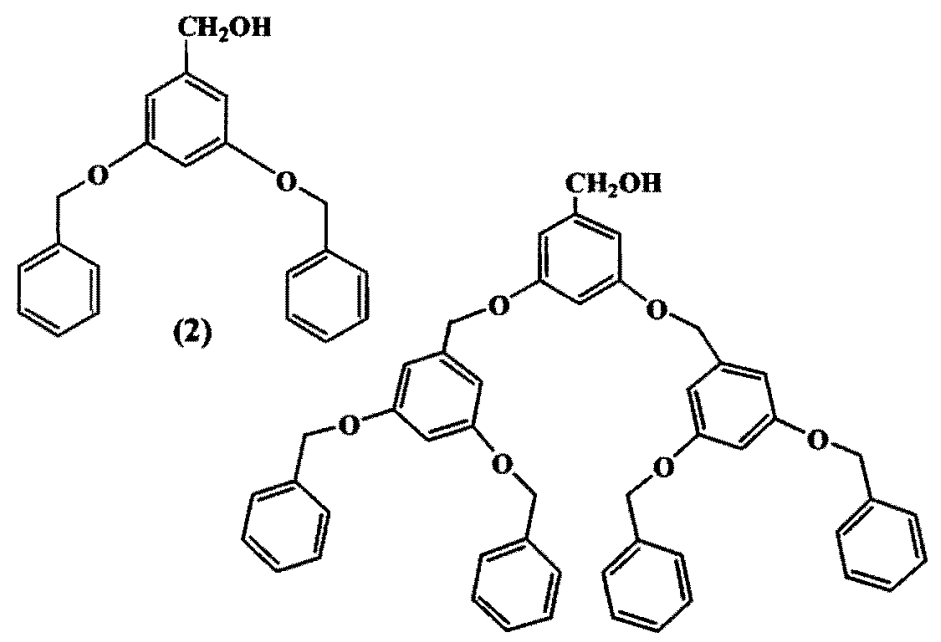

(5)

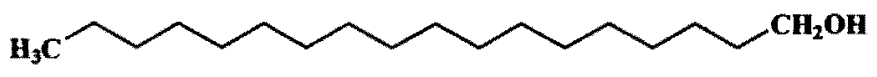

(6)<smiles>OCc1ccncc1</smiles>

(7)<smiles>OCc1ccccc1</smiles>

(8)

Figure 4-3. The chemical structures of the desired poly(benzyl ether) dendrimers and other alcohol materials. 

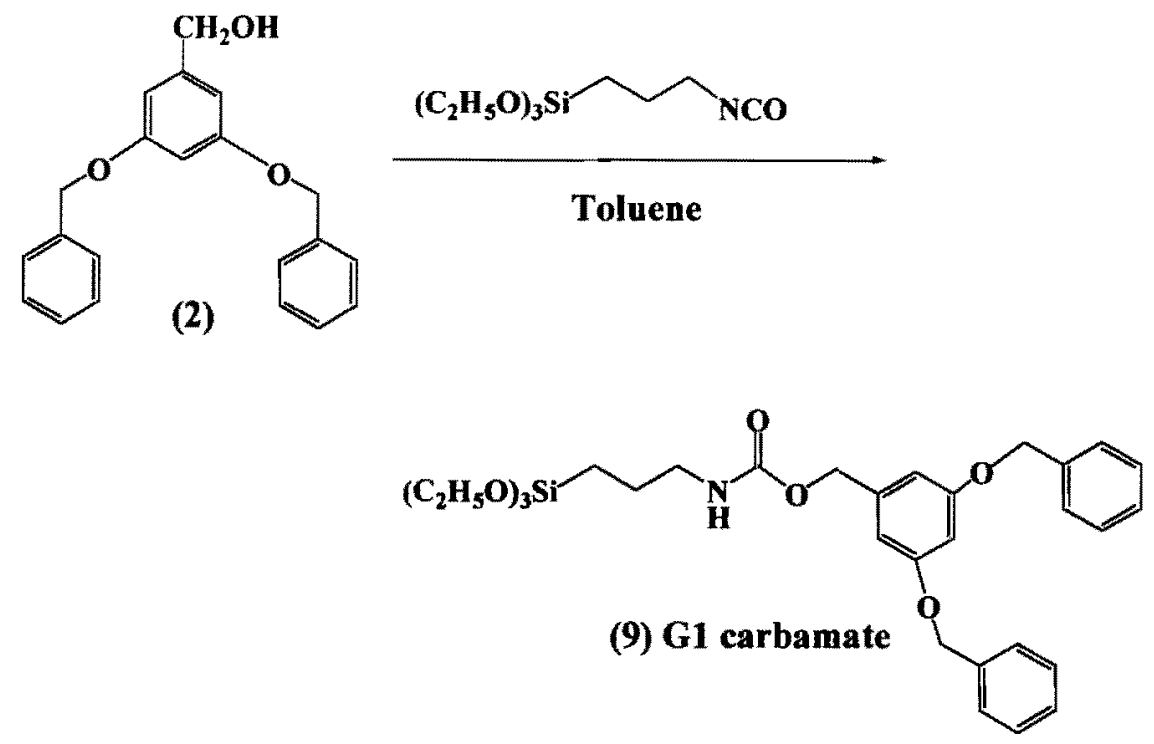

Figure 4-4. Synthesis scheme illustrating the preparation of carbamates from the coupling reaction.

4.4.1 General procedure: The appropriate alcohol $(2,5,6,7$, or 8$)(1.10$ eq.) was dissolved in anhydrous toluene (other suitable solvents may also be used, refer to individual experiment). 3-(triethoxysilyl)propyl isocyanate (1.0 eq.) was mixed with a minimum amount of anhydrous toluene to prevent rapid hydrolysis of the isocyanate. The mixture was added slowly using an addition funnel to the alcohol with constant stirring and a nitrogen blanket. When the addition was complete, the reaction was allowed to reflux for 18 hours. The solvent was then removed in vacuo to give the carbamate. The carbamate was used to coat fused silica capillary columns without further purification. 


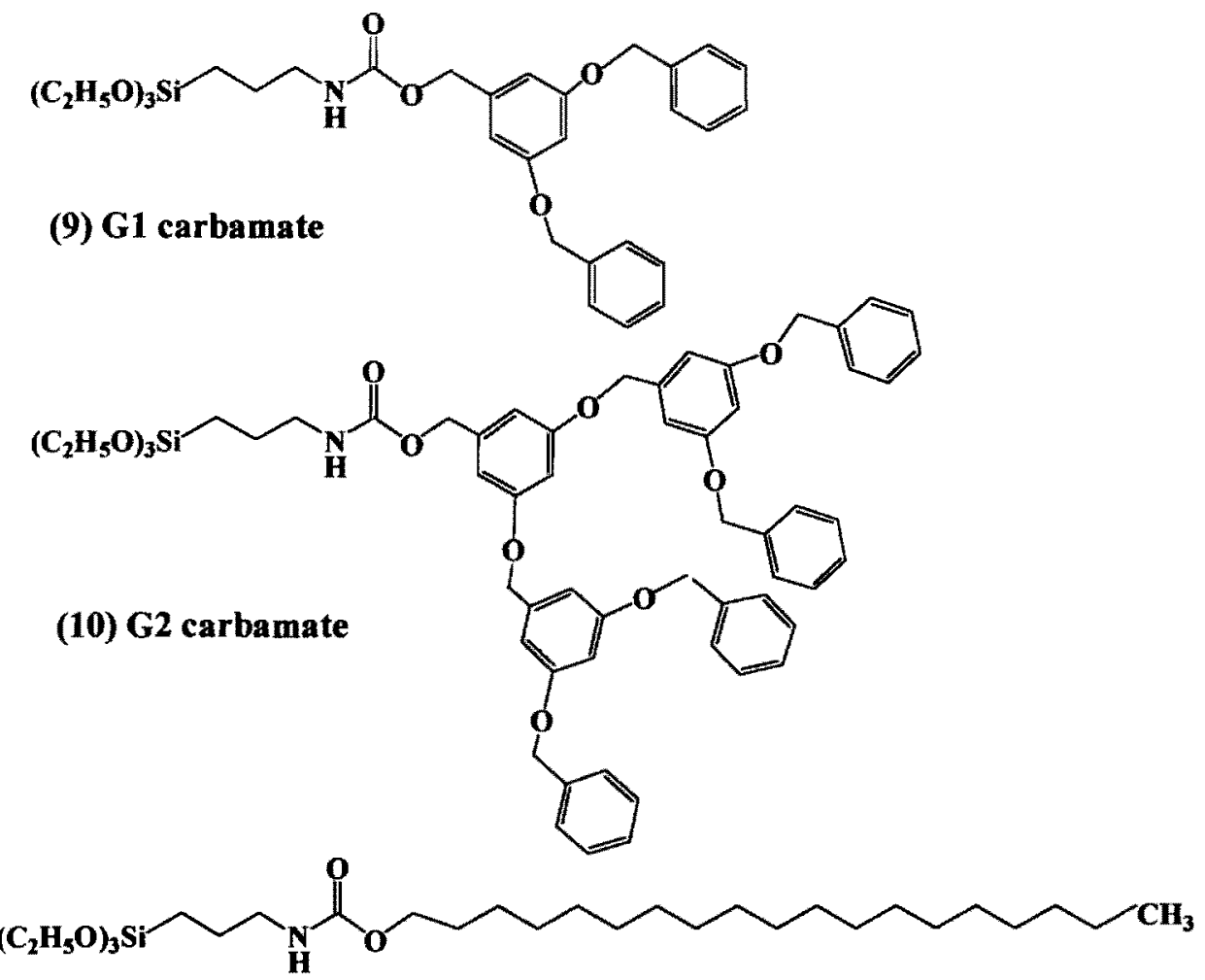

(11) C18 carbamate<smiles>CCOCCCCNC(=O)OCc1ccncc1</smiles>

(12) Pyridyl carbinol carbamate<smiles>CCCCCCCCCCCCCCCCCOC(=O)NCc1ccccc1</smiles>

(13) Benzyl carbamate

Figure 4-5. The chemical structures of the carbamates used as coating materials for CEC evaluation. 


\subsubsection{3, 5-dibenzyloxybenzyl 3-(triethoxysilyl)propyl carbamate (9), G1 carbamate.}

$\mathrm{C}_{31} \mathrm{H}_{41} \mathrm{NO}_{7} \mathrm{Si}$. Mol. Wt. 567.37. Reaction of 2,31.82 g, (99.4 mmol, 1.10 eq.) with 3(triethoxysilyl)propyl isocyanate, $22.27 \mathrm{~g},(90.2 \mathrm{mmol}, 1.0 \mathrm{eq}$.) in $200 \mathrm{~mL}$ of anhydrous toluene proceeded by the general procedure described above to give carbamate 9 . The reaction was monitored by both TLC and isocratic HPLC: $20 \% \mathrm{dd}_{2} \mathrm{O}, 80 \%$ acetonitrile, product $t_{\mathrm{r}}=12.2 \mathrm{~min}$. FTIR ( $\mathrm{NaCl}$ plate): $3341 \mathrm{~cm}^{-1}\left(\mathrm{NH}\right.$, as, st), $3041 \mathrm{~cm}^{-1}(\mathrm{Ar} \mathrm{C}-\mathrm{H}$, st), $2993 \mathrm{~cm}^{-1}$ (CH, aliph., st), $1723 \mathrm{~cm}^{-1}(\mathrm{C}=\mathrm{O}, \mathrm{st}), 1580 \mathrm{~cm}^{-1}(\mathrm{~N}-\mathrm{H}$, deformation), $1458 \mathrm{~cm}^{-1}\left(-\mathrm{CH}_{3}\right.$, as., bending), and $1085 \mathrm{~cm}^{-1}\left(\mathrm{Si}-\mathrm{O}-\mathrm{C}_{2} \mathrm{H}_{5}\right.$, st, intensive doublet). ${ }^{1} \mathrm{H}-\mathrm{NMR}\left(\mathrm{CDCl}_{3}, \mathrm{TMS}\right): \delta 7.402\left(\mathrm{~m}, \mathrm{Ar}_{1} \mathrm{H}, 10 \mathrm{H}\right), 6.599\left(\mathrm{~s}, \mathrm{Ar}_{0} \mathrm{H}, \mathrm{C}_{2,6}-\mathrm{H}, 2 \mathrm{H}\right) ; 6.556$ (s, $\left.\mathrm{Ar}_{0} \mathrm{H}, \mathrm{C}_{4}-\mathrm{H}, 1 \mathrm{H}\right), 5.022\left(\mathrm{~s}, \mathrm{Ar}_{0} \mathrm{H},-\mathrm{CH}_{2}-\mathrm{O}, 2 \mathrm{H}\right.$, and $\mathrm{Ar}_{1} \mathrm{H},-\mathrm{CH}_{2}-\mathrm{O}, 4 \mathrm{H}$ ), 3.820 (m, $\left.\mathrm{CH}_{2} \mathrm{NH}, 2 \mathrm{H}\right), 1.652\left(\mathrm{~m}, \mathrm{CH}_{2} \mathrm{CH}_{2} \mathrm{CH}_{2} \mathrm{NH}, 2 \mathrm{H}\right), 1.23\left(\mathrm{~m},\left(\mathrm{CH}_{3} \mathrm{CH}_{2} \mathrm{O}\right)_{3} \mathrm{Si}, 9 \mathrm{H}\right)$ and 0.63 (m, $\mathrm{OSiCH}_{2} \mathrm{CH}_{2}, 2 \mathrm{H}$ ). ESI mass spectrum of 3, 5-dibenzyloxybenzyl 3(triethoxysilyl)propyl carbamate did not exhibit the $[\mathrm{M}+\mathrm{H}]^{+}$568. Instead the diagnostic loss of $\mathrm{C}_{2} \mathrm{H}_{5} \mathrm{OH}$ was observed resulting in the $\mathrm{m} / \mathrm{z} 522$ fragment. An additional loss of ethanol was found when the $\mathrm{m} / \mathrm{z} 469$ eliminates ethanol to give $\mathrm{m} / \mathrm{z} 423$. The complimentary $\mathrm{m} / \mathrm{z} 303$ (3, 5-dibenzyloxybenzylic fragment) is observed with the protonated 3-(triethoxysilyl)propyl isocyanate ion, $\mathrm{m} / \mathrm{z} 248$. Elemental analysis calculated for $\mathrm{C}_{31} \mathrm{H}_{41} \mathrm{NO}_{7} \mathrm{Si}: \mathrm{C}, 65.58 ; \mathrm{H}, 7.28 ; \mathrm{N}, 2.47 ; \mathrm{Si}, 4.94$, found: $\mathrm{C}, 64.12 ; \mathrm{H}$, $7.45 ; \mathrm{N}, 2.87 ; \mathrm{Si}, 4.70$.

\subsubsection{3, 5-(3', 5'-benzyloxybenzyloxy) benzyl 3-(triethoxysilyl)propyl carbamate} (10), G2 carbamate. $\mathrm{C}_{59} \mathrm{H}_{65} \mathrm{NO}_{11} \mathrm{Si}$. Mol. Wt. 991.37 . Product 5, $8.184 \mathrm{~g},(11.0 \mathrm{mmol}$, 1.10 eq.), 3-(triethoxysilyl)propyl isocyanate, $2.23 \mathrm{~g},(9.02 \mathrm{mmol}, 1.0 \mathrm{eq}$.$) and 100 \mathrm{~mL}$ of anhydrous toluene were treated by the general procedure described above to give 
carbamate 10. The reaction was monitored by both TLC and isocratic HPLC: $20 \%$ dd $\mathrm{H}_{2} \mathrm{O}, 80 \%$ acetonitrile, product $\mathrm{t}_{\mathrm{r}}=12.2 \mathrm{~min}$. Elemental analysis calculated for $\mathrm{C}_{59} \mathrm{H}_{65} \mathrm{NO}_{11} \mathrm{Si}: \mathrm{C}, 71.42 ; \mathrm{H}, 6.60 ; \mathrm{N}, 1.41 ; \mathrm{Si}, 2.83$, found: $\mathrm{C}, 71.07 ; \mathrm{H}, 6.81 ; \mathrm{N}, 1.56$; $\mathrm{Si}, 2.91$.

\subsubsection{1-Octadecyl 3-(triethoxysilyl)propyl carbamate (11), Stearyl Carbamate.} $\mathrm{C}_{28} \mathrm{H}_{59} \mathrm{NO}_{5} \mathrm{Si}$, Mol. Wt. 517.87. Stearyl alcohol, 6, $21.28 \mathrm{~g}$, (100.85 mmol, 1.01 eq.) 3(triethoxysilyl)propyl isocyanate, $24.68 \mathrm{~g},(99.76 \mathrm{mmol}, 1.0$ eq.) and $100 \mathrm{~mL}$ of anhydrous THF were treated by the general procedure described above to give carbamate 11. A clear solution was observed after 20 minutes reflux. FTIR spectroscopy was used to monitor the reaction kinetics. Samples were taken at 2, 5, 9, and 21 hours to ensure completion of the reaction. The reaction was allowed to reflux up to 48 hours. The removal of the solvent in vacuo gave a white waxy powder. FTIR ( $\mathrm{KBr}$ pellet): $3221 \mathrm{~cm}^{-1}(\mathrm{~N}-\mathrm{H}, \mathrm{st}), 2922 \mathrm{~cm}^{-1}$ (C-H aliphatic, st), $1687 \mathrm{~cm}^{-1}(-\mathrm{C}=\mathrm{O}, \mathrm{st}), 1548 \mathrm{~cm}^{-1}$ ( $\mathrm{N}-\mathrm{H}$ deformation), $1102 \mathrm{~cm}^{-1} ; 1081 \mathrm{~cm}^{-1}\left(\mathrm{Si}-\mathrm{O}-\mathrm{CH}_{2}-\mathrm{CH}_{3}\right)$, st, intense doublet), and $726 \mathrm{~cm}^{-1}$ (Si-C, st). ${ }^{1} \mathrm{H}-\mathrm{NMR}\left(\mathrm{CDCl}_{3}, \mathrm{TMS}\right): \delta 4.885$ (s, broad, $\left.\mathrm{O}=\mathrm{C}-\mathrm{N} \underline{\mathrm{H}}-\mathrm{CH}_{2}, 1 \mathrm{H}\right)$, $4.051\left(\mathrm{~m}, \mathrm{comb} ; \mathrm{CH}_{2}-\mathrm{O}-\mathrm{C}=\mathrm{O}, 2 \mathrm{H}\right), 3.832\left(\mathrm{q}, \mathrm{Si}-\left(\mathrm{O}-\mathrm{CH}_{2}-\mathrm{CH}_{3}\right)_{3}, 6 \mathrm{H}\right), 3.183$ (q, $\left.\mathrm{O}=\mathrm{C}-\mathrm{NH}-\mathrm{CH}_{2}-, 2 \mathrm{H}\right), 1.645\left(\mathrm{~m}, \mathrm{NH}-\mathrm{CH}_{2}-\mathrm{CH}_{2}-\mathrm{CH}_{2}-\mathrm{Si}, 2 \mathrm{H}\right), 1.255$ (t, comb. Si- $\left.\left(\mathrm{O}-\mathrm{CH}_{2}-\mathrm{CH}_{3}\right)_{3}, 9 \mathrm{H} ; \mathrm{CH}_{3}-\left(\mathrm{CH}_{2}\right)_{16}-\mathrm{CH}_{2}-\mathrm{O}, 32 \mathrm{H}\right), 0.880\left(\mathrm{t}, \underline{\mathrm{CH}_{2}}-\left(\mathrm{CH}_{2}\right)_{17}, 3 \mathrm{H}\right)$, and 0.657 (t, $\left.-\mathrm{CH}_{2}-\mathrm{Si}, 2 \mathrm{H}\right)$. Elemental analysis calculated for $\mathrm{C}_{28} \mathrm{H}_{59} \mathrm{NO}_{5} \mathrm{Si}$ : C, 64.94; $\mathrm{H}, 11.48 ; \mathrm{N}, 2.70 ; \mathrm{Si}, 5.42$, found: $\mathrm{C}, 65.28 ; \mathrm{H}, 11.54 ; \mathrm{N}, 2.60 ; \mathrm{Si}, 5.54$.

4.4.5 4-pyridyl carbinol 3-(triethoxysilyl)propyl carbamate (12). $\mathrm{C}_{16} \mathrm{H}_{28} \mathrm{~N}_{2} \mathrm{O}_{5} \mathrm{Si}$, 356.47. 4-pyridyl carbinol 7, $58.63 \mathrm{~g},(0.54 \mathrm{~mole}, 1.08 \mathrm{eq}$.) and 3-(triethoxysilyl)propyl isocyanate, $123.36 \mathrm{~g},(0.50$ mole, $1.0 \mathrm{eq}$.$) in 200 \mathrm{~mL}$ of anhydrous methyl t-butyl ether 
were reacted by the general procedure described above to give carbamate 12. Upon the completion of addition, the reaction was refluxed for 12 hours under nitrogen. TLC showed a single spot for the product. A clear liquid residue was obtained when the solvent was removed in vacuo. FTIR (thin film $\mathrm{NaCl}$ plate): $3403 \mathrm{~cm}^{-1}(\mathrm{~N}-\mathrm{H}, \mathrm{st}$ ), $3064 \mathrm{~cm}^{-1}$ (pyr C-H, st), $1720 \mathrm{~cm}^{-1}$ (C=O, st), $1565 \mathrm{~cm}^{-1}$ (N-H, deformation), $1457 \mathrm{~cm}^{-1}$ (C-H, as bending), $1085 \mathrm{~cm}^{-1}$ (Si-O- $\mathrm{C}_{2} \mathrm{H}_{5}$, st, intense doublet). ${ }^{1} \mathrm{H}-\mathrm{NMR}\left(\mathrm{CDCl}_{3}\right.$, TMS): $\delta 8.568$ (m, Pyr, $\left.\underline{\mathrm{C}_{2.6}-\mathrm{H}}, 2 \mathrm{H}\right), 7.232\left(\mathrm{~m}, \mathrm{Pyr}, \underline{\mathrm{C}_{3.5}-\mathrm{H}}, 2 \mathrm{H}\right), 5.109(\mathrm{~m},-\mathrm{N} \underline{\mathrm{H}}-\mathrm{C}=\mathrm{O}$, 1H), 3.8112 (q, $\left.\mathrm{CH}_{3}-\mathrm{CH}_{2}-\mathrm{O}-\mathrm{Si}, 6 \mathrm{H}\right), 3.232\left(\mathrm{q},-\mathrm{CH}_{2}-\mathrm{N}, 2 \mathrm{H}\right), 2.354\left(\mathrm{~s}, \mathrm{O}-\mathrm{CH}_{2}-\mathrm{Pyr}\right.$, $2 \mathrm{H}), 1.657\left(\mathrm{~m},-\mathrm{CH}_{2}-\mathrm{CH}_{2}-\mathrm{NH}, 2 \mathrm{H}\right), 1.226\left(\mathrm{t}, \mathrm{CH}_{3}-\mathrm{CH}_{2}-\mathrm{O}, 9 \mathrm{H}\right)$, and $0.6\left(\mathrm{~m}, \mathrm{Si}-\mathrm{CH}_{2}-\right.$, 2H). ESI mass spectrum: 4-pyridyl carbinol 3-(triethoxysilyl)propyl carbamate $[\mathrm{M}+\mathrm{H}]+$, 357 , with the facile loss of $\mathrm{C}_{2} \mathrm{H}_{5} \mathrm{OH}$ to give the $\mathrm{m} / \mathrm{z}=311$. Elemental analysis: calculated for $\mathrm{C}_{16} \mathrm{H}_{28} \mathrm{~N}_{2} \mathrm{O}_{5} \mathrm{Si}: 356.47, \mathrm{C}, 53.91 ; \mathrm{H}, 7.92 ; \mathrm{N}, 7.86 ; \mathrm{Si}, 7.88$; found: $\mathrm{C}, 56.65 ; \mathrm{H}, 7.81$; $\mathrm{N}, 7.14 ; \mathrm{Si}, 7.79$.

Note: The ${ }^{1} \mathrm{H}-\mathrm{NMR}$ spectrum ( $\mathrm{CDCl}_{3}$, TMS) shows some impurities, and the mass spectrum contains an unknown $[\mathrm{M}+\mathrm{H}]^{+}$of 420 .

4.4.6 Benzyl 3-(triethoxysilyl)propyl carbamate (13). $\mathrm{C}_{17} \mathrm{H}_{29} \mathrm{NO}_{5} \mathrm{Si}$, Mol. Wt. 355.51. Benzyl alcohol 8, $47.84 \mathrm{~g},(0.44 \mathrm{~mole}, 1.10 \mathrm{eq}$.) and 3-(triethoxysilyl)propyl isocyanate, $99.75 \mathrm{~g},(0.40$ mole, $1.0 \mathrm{eq}$.) in $100 \mathrm{~mL}$ of anhydrous methyl t-butyl ether were reacted by the general procedure described above to give carbamate 13. The reaction was refluxed for 12 hours under $\mathrm{N}_{2}$. A TLC method was used to monitor the reaction. A clear liquid residue was obtained after the removal of the solvent in vacuo. FTIR (thin film on $\mathrm{NaCl}$ plate): $3343 \mathrm{~cm}^{-1}$ (NH, st), $3090 \mathrm{~cm}^{-1}$ (ArH, as st), $3033 \mathrm{~cm}^{-1}$ (ArH, sy, st), $2975 \mathrm{~cm}^{-1}$ ( $\mathrm{CH}_{2}$, as vib), $2886 \mathrm{~cm}^{-1}\left(\mathrm{CH}_{2}\right.$, sy vib), $1705 \mathrm{~cm}^{-1}(\mathrm{C}=\mathrm{O}$, st), 
$1529 \mathrm{~cm}^{-1}$ (NH, deformation), $1455 \mathrm{~cm}^{-1}$ (as $\mathrm{CH}$ deformation, $\mathrm{CH}_{3}$ ), $1390 \mathrm{~cm}^{-1}$ (sy $\mathrm{CH}$ deformation, $\mathrm{CH}_{3}$ ), $1103 \mathrm{~cm}^{-1} ; 1079 \mathrm{~cm}^{-1}$ (SiO, st intensed; $\mathrm{SiOC}_{2} \mathrm{H}_{5}$ ), $776 \mathrm{~cm}^{-1} ; 698 \mathrm{~cm}^{-1}$ ( $\mathrm{Ar}, \mathrm{CH}$ out of plane bending, characteristic of monosubstitution). ${ }^{1} \mathrm{H} \mathrm{NMR}\left(\mathrm{CDCl}_{3}\right.$, TMS): $\delta 7.35(\mathrm{~m}, \mathrm{Ar} \underline{\mathrm{H}}, 5 \mathrm{H}), 5.091\left(-\mathrm{O}-\mathrm{C}_{\underline{2}}-\mathrm{Ar}, 2 \mathrm{H}\right), 5.005(-\mathrm{N} \underline{\mathrm{H}}-\mathrm{C}=\mathrm{O}, 1 \mathrm{H}), 3.845(\mathrm{q}$, $\left.\mathrm{Si}-\left(\mathrm{OCH}_{2} \mathrm{CH}_{3}\right)_{3}, 6 \mathrm{H}\right), 3.188\left(\mathrm{~m},-\mathrm{CH}_{2}-\mathrm{NH}, 2 \mathrm{H}\right), 1.683\left(\mathrm{~m},-\mathrm{CH}_{2}-\mathrm{CH}_{2}-\mathrm{NH}, 2 \mathrm{H}\right), 1.217$ (t, $\left.\mathrm{Si}-\left(\mathrm{O}-\mathrm{CH}_{2}-\mathrm{CH}_{3}\right)_{3}, 9 \mathrm{H}\right)$, and $0.628\left(\mathrm{t}, \mathrm{Si}-\mathrm{CH}_{2} \mathrm{CH}_{2}, 2 \mathrm{H}\right)$. ESI mass spectrum: benzyl alcohol carbamate $[\mathrm{M}+\mathrm{H}]+, 356$. The mass spectrum also contains a $\mathrm{m} / \mathrm{z}, 310$ fragment due to the loss of ethanol. Elemental analysis: calculated for $\mathrm{C}_{17} \mathrm{H}_{29} \mathrm{NO}_{5} \mathrm{Si}$ : $\mathrm{C}, 57.44$; $\mathrm{H}, 8.22 ; \mathrm{N}, 3.94 ; \mathrm{Si}, 7.90 ;$ found: $57.92 ; \mathrm{H}, 8.26 ; \mathrm{N}, 3.84 ; \mathrm{Si}, 7.80$.

\subsection{Mass spectrometry results}

Table 4-1 LC/MS Electrospray Ionization Data for the Compounds Investigated

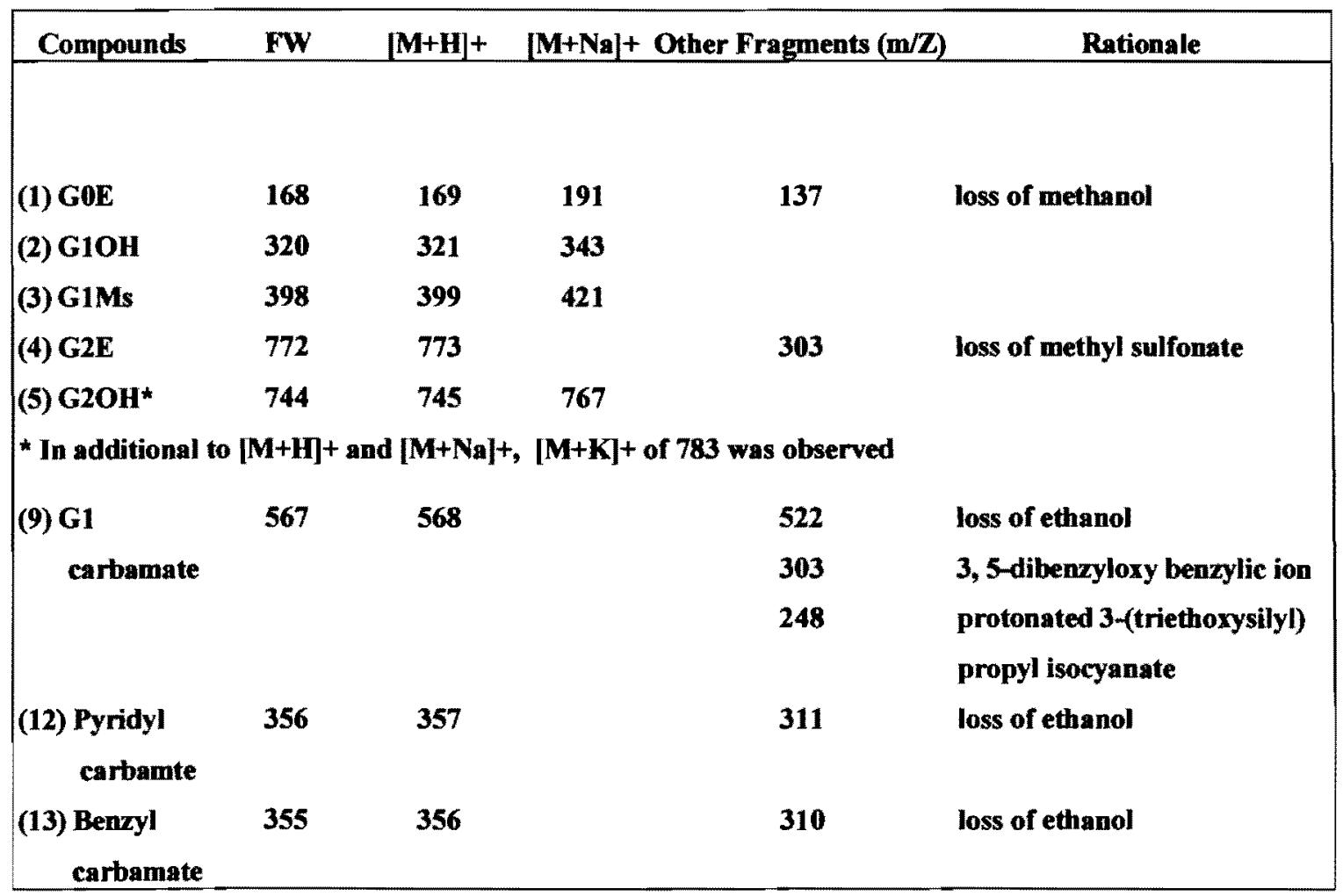




\subsection{Collection of Spectral Data}

$\begin{array}{ll}\text { Mass Spectra } & \text { - page } 106 \text { to } 111 . \\ { }^{1} \text { NMR Spectra } & \text { - page } 113 \text { to } 122 . \\ \text { FTIR Spectra } & \text { - page } 124 \text { to } 131 .\end{array}$




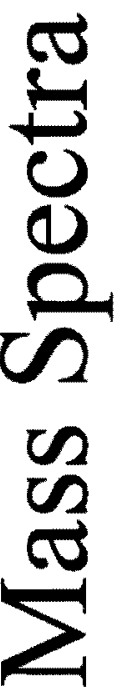




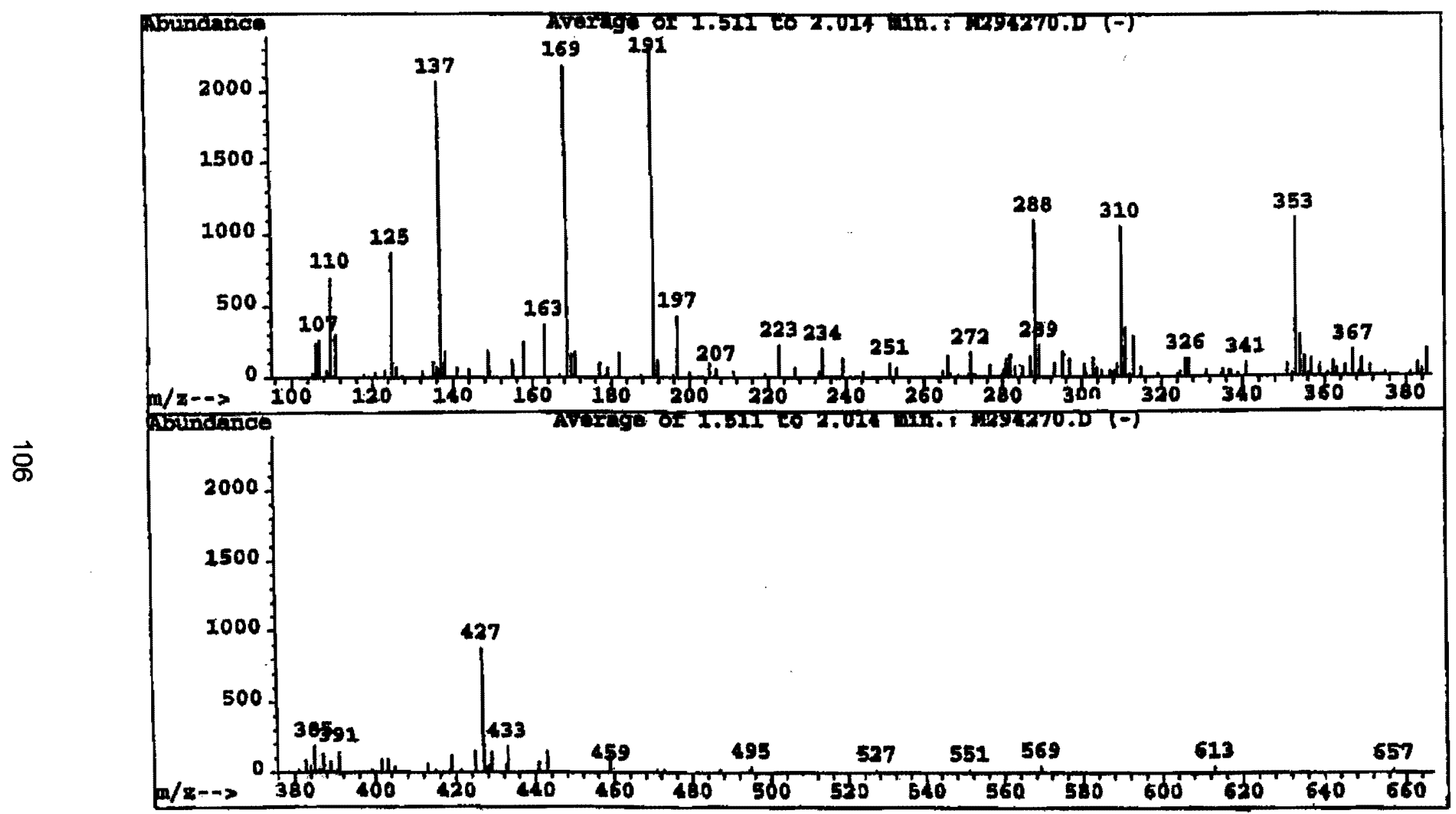

Figure 4-6. Mas spectrum of methyl 3, 5-dihydroxybenzoate (1). 


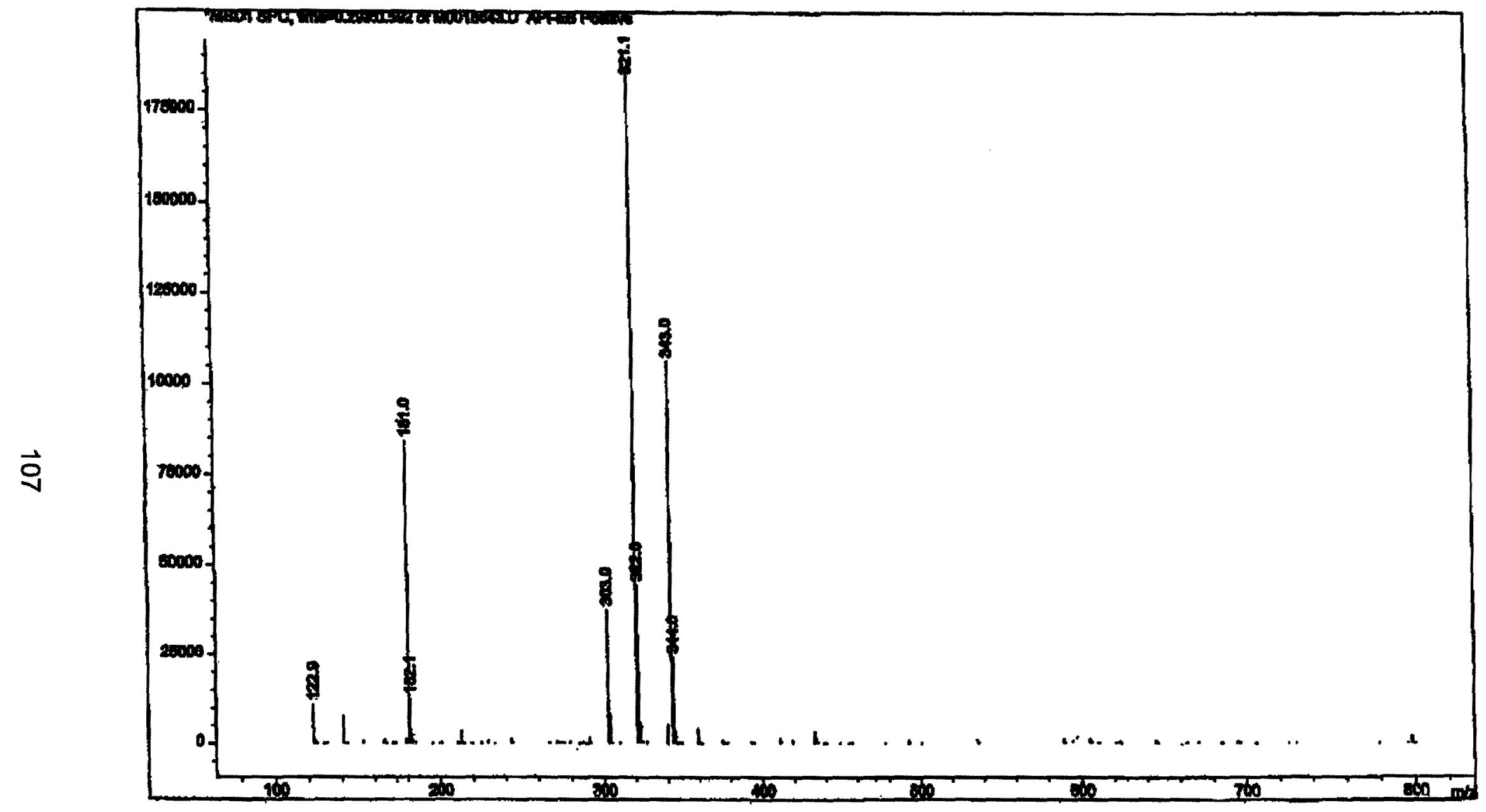

Figure 4-7. Mass spectrum of 3, 5-dibenzyloxybenzylalcohol (2). 


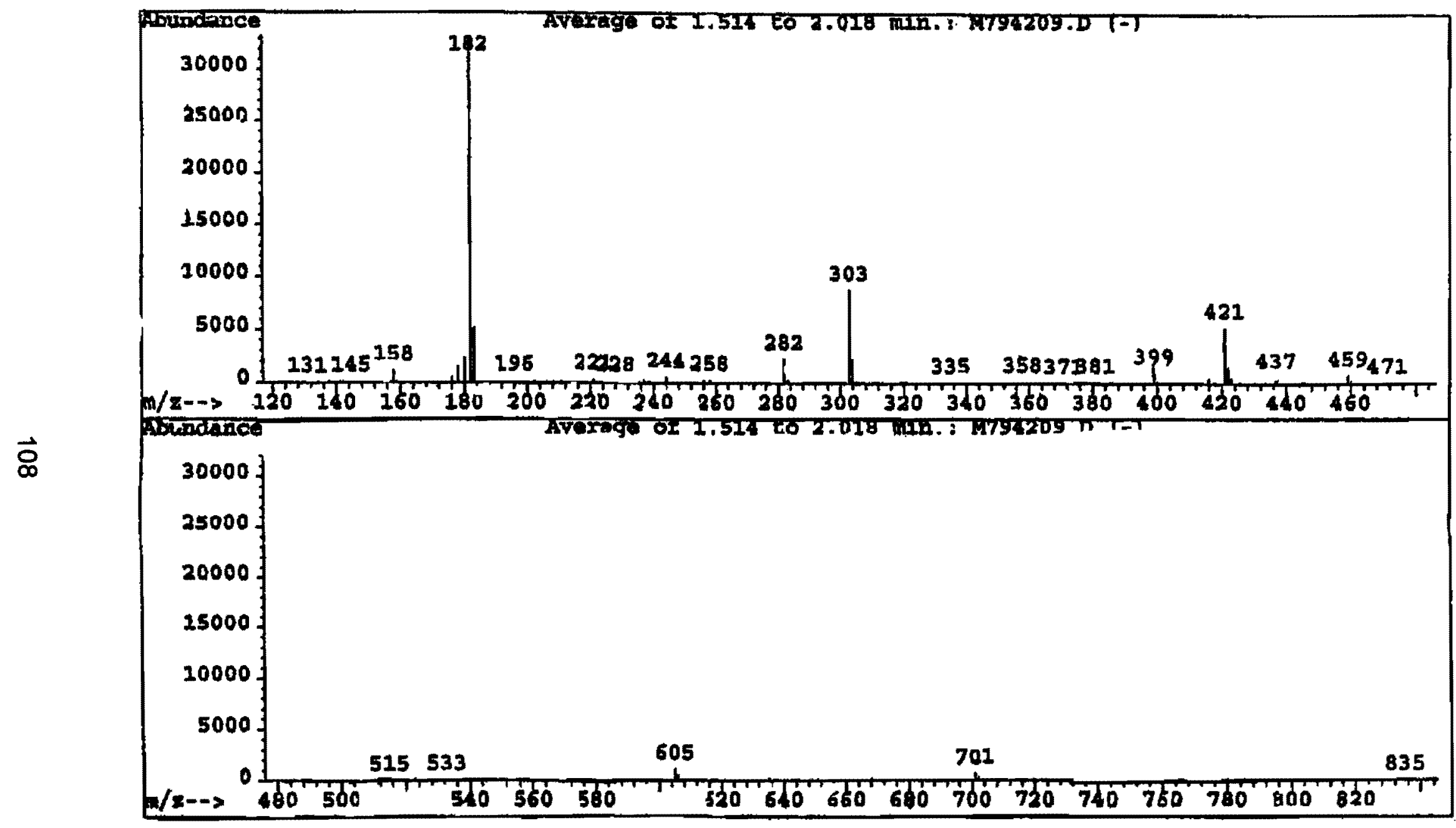

Figure 4-8. Mass spectrum of 3,5-dibenzyloxy benzyl methane sulphonate (3). 


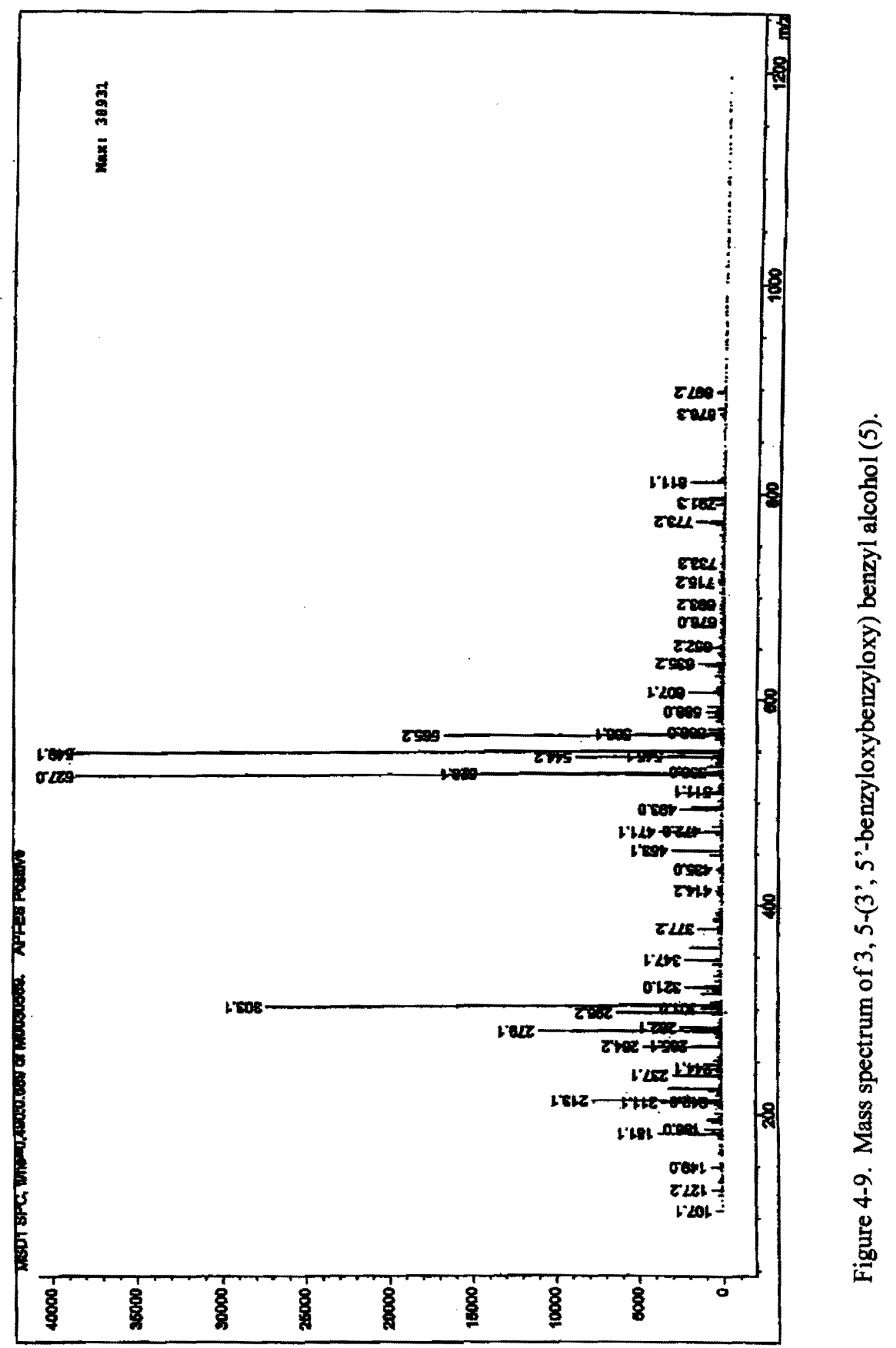




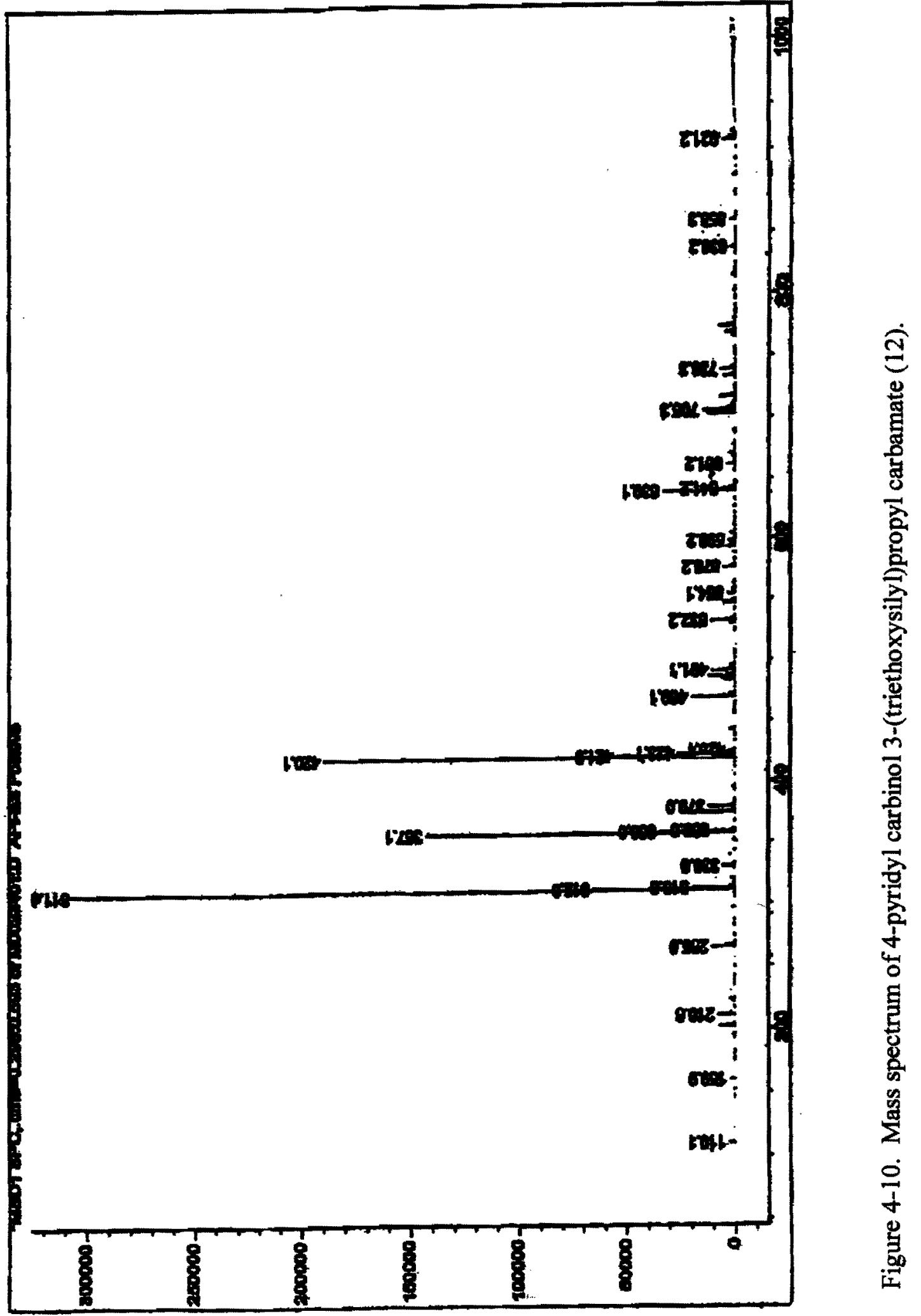




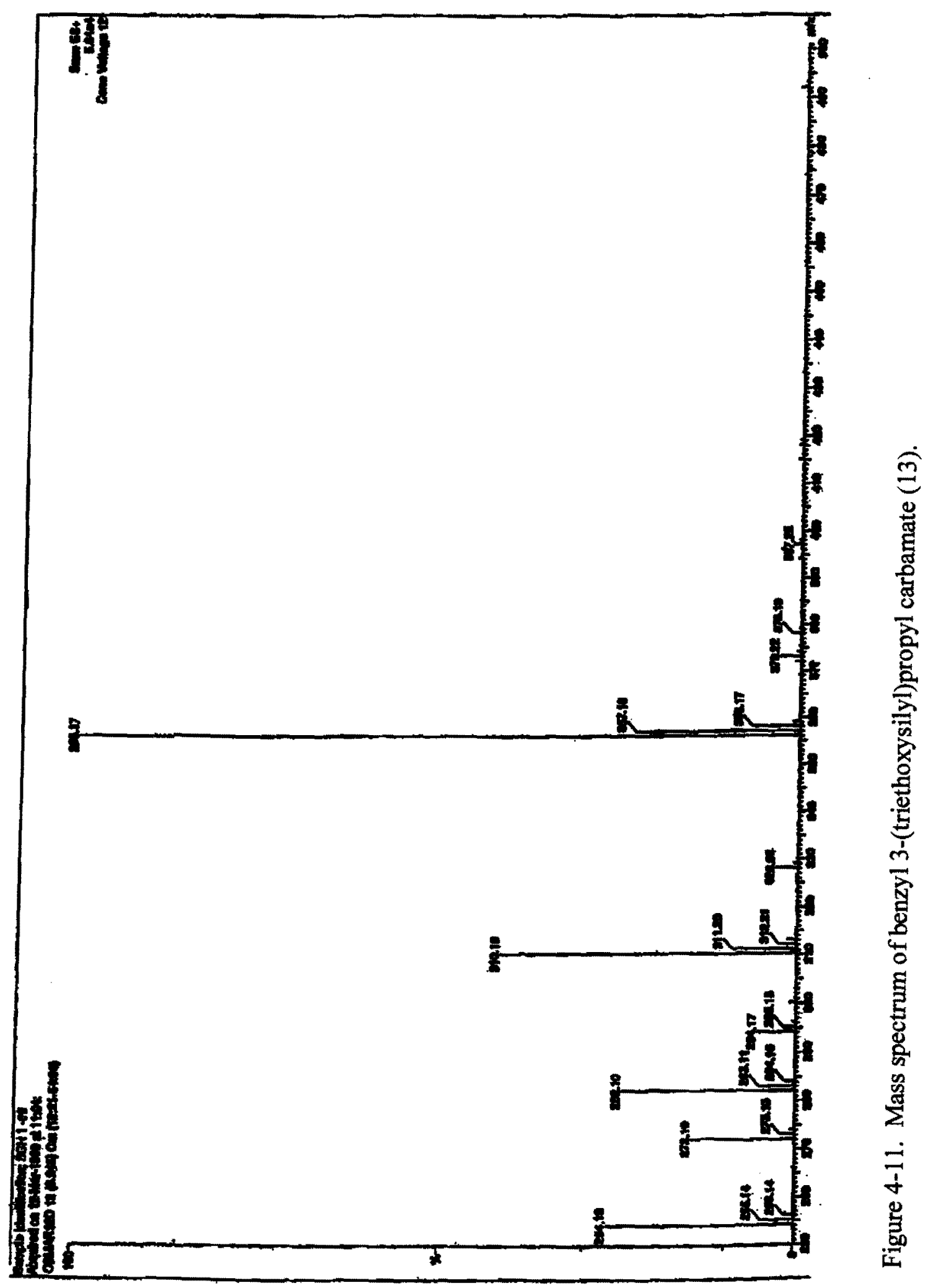




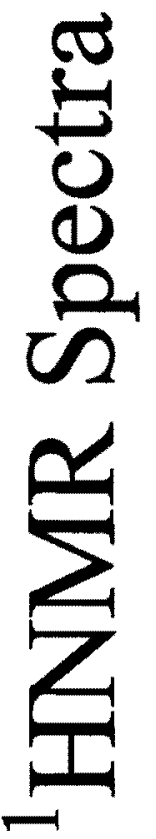




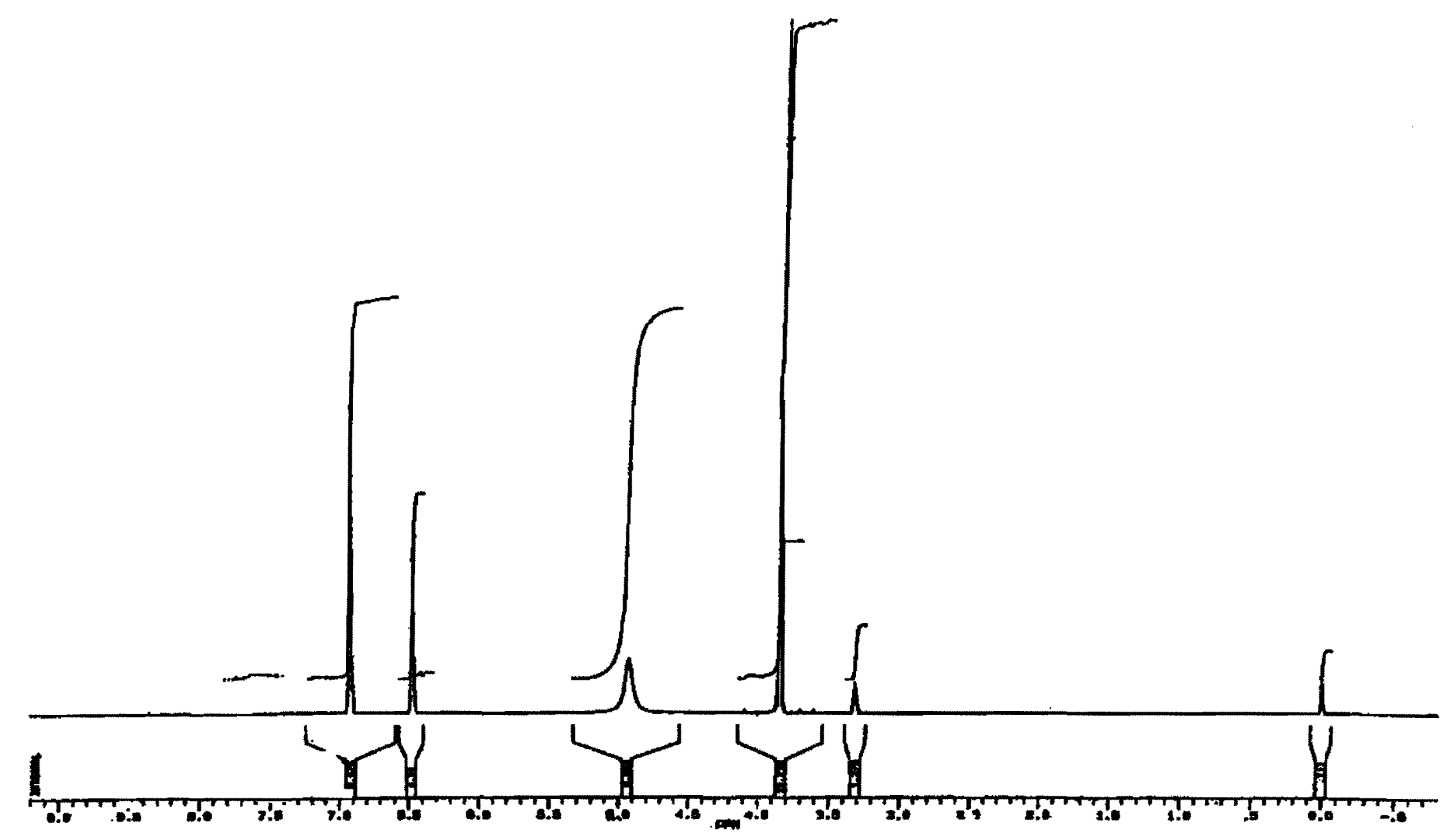

Figure 4-12. $300-\mathrm{MHz}{ }^{1} \mathrm{H}$ NMR spectrum of methyl 3, 5-dihydroxybenzoate (1). 


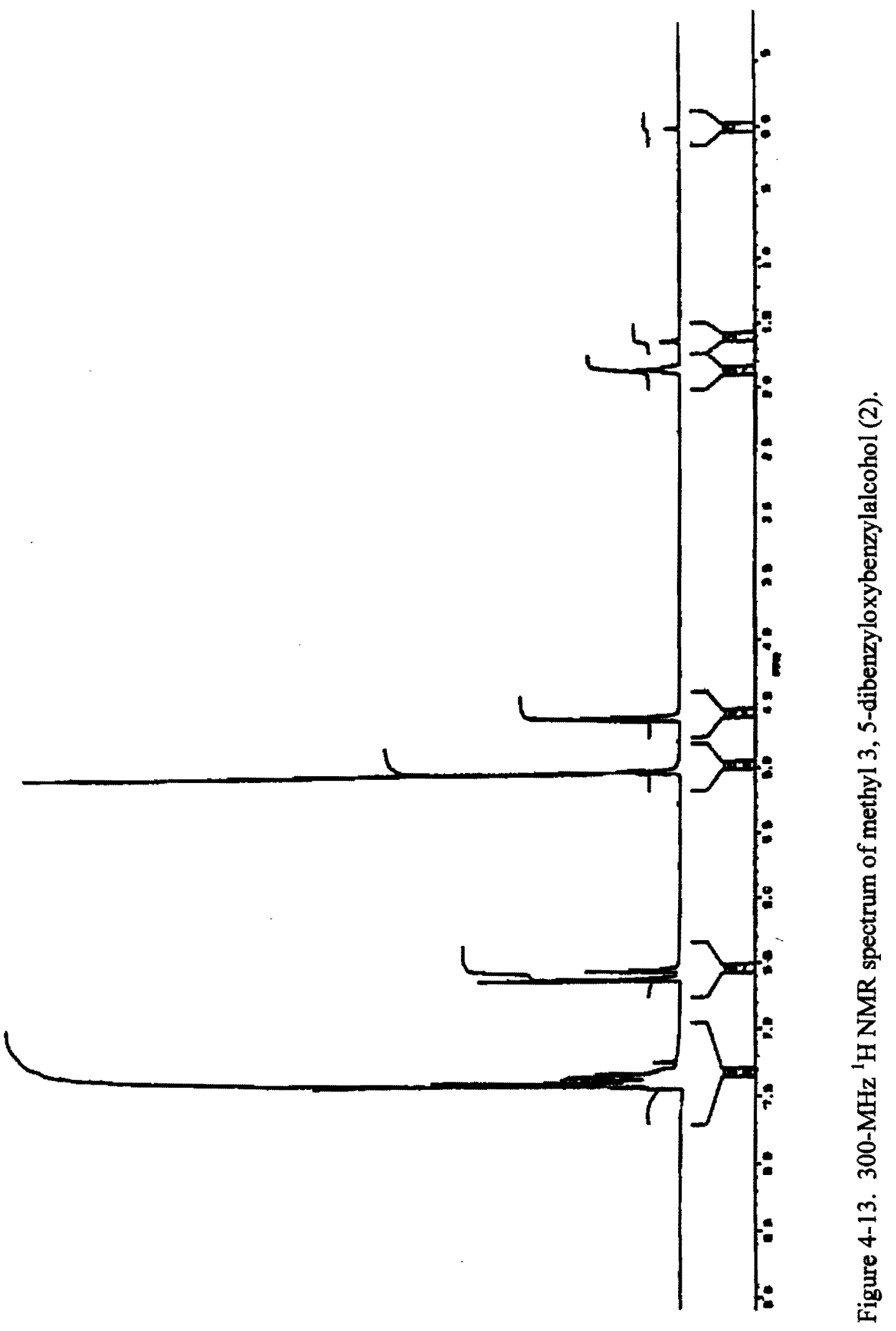




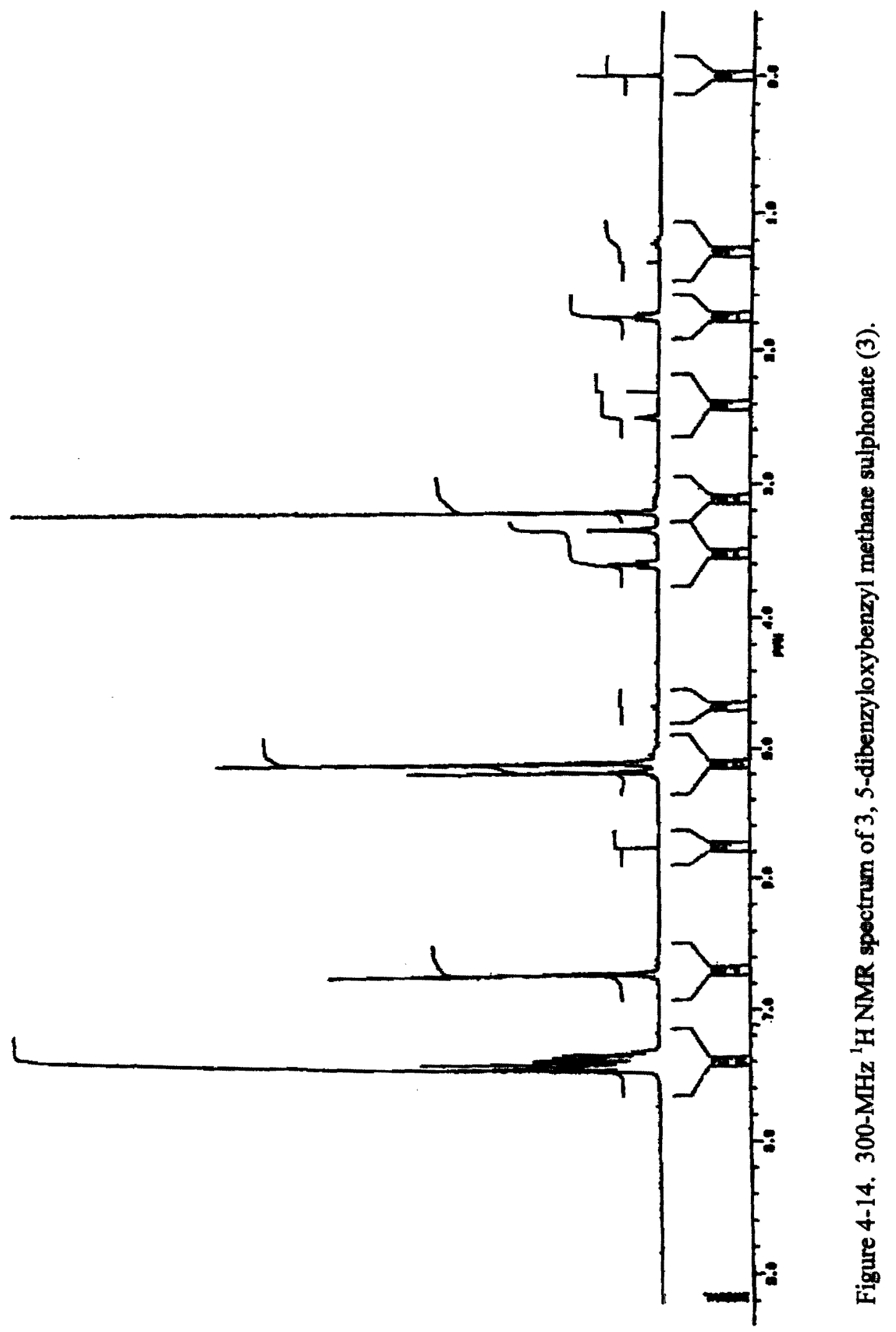


$\overrightarrow{\vec{\sigma}}$

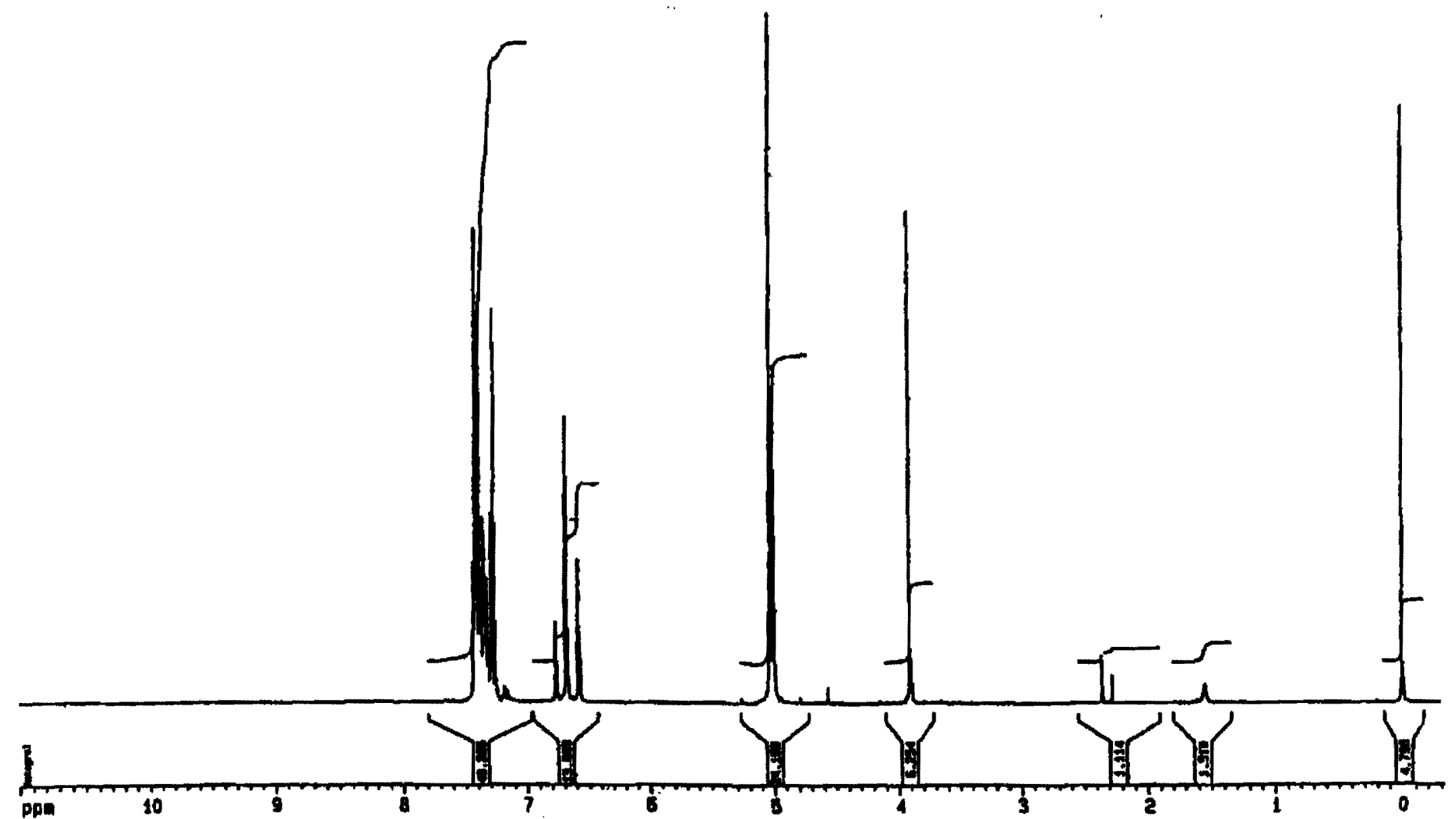

Figure 4-15. 300-MHz ${ }^{1} \mathrm{H}$ NMR spectrum of methyl 3, 5-(3', 5'-benzyloxybenzyloxy)benzoate (4). 
$H_{1}$ 


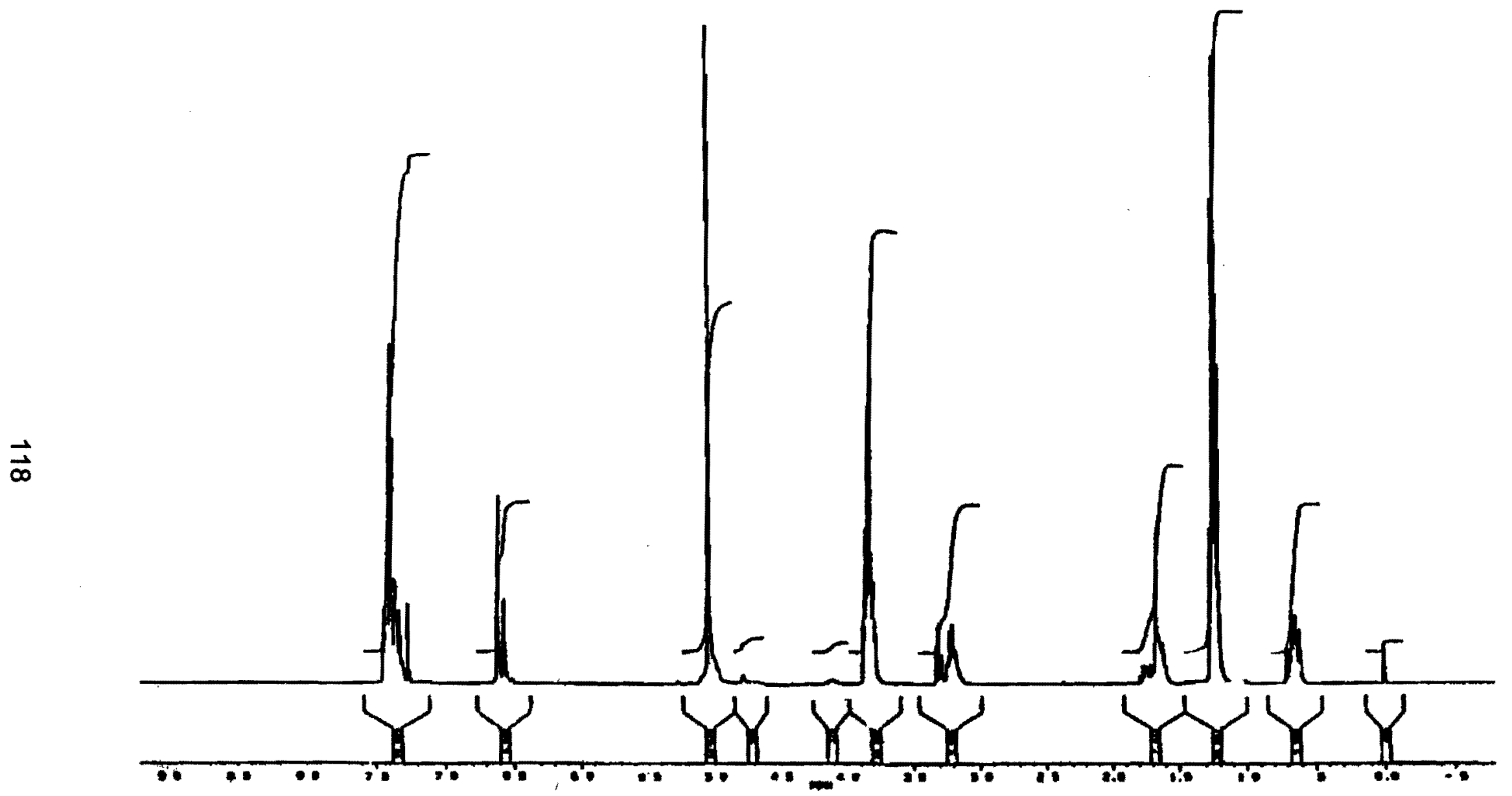

Figure 4-17. 300-MHz ${ }^{1} \mathrm{H}$ NMR spectrum of 3, 5-dibenzyloxybenzyl 3-(triethoxysilyl)propyl carbamate (9), G1 carbamate. 


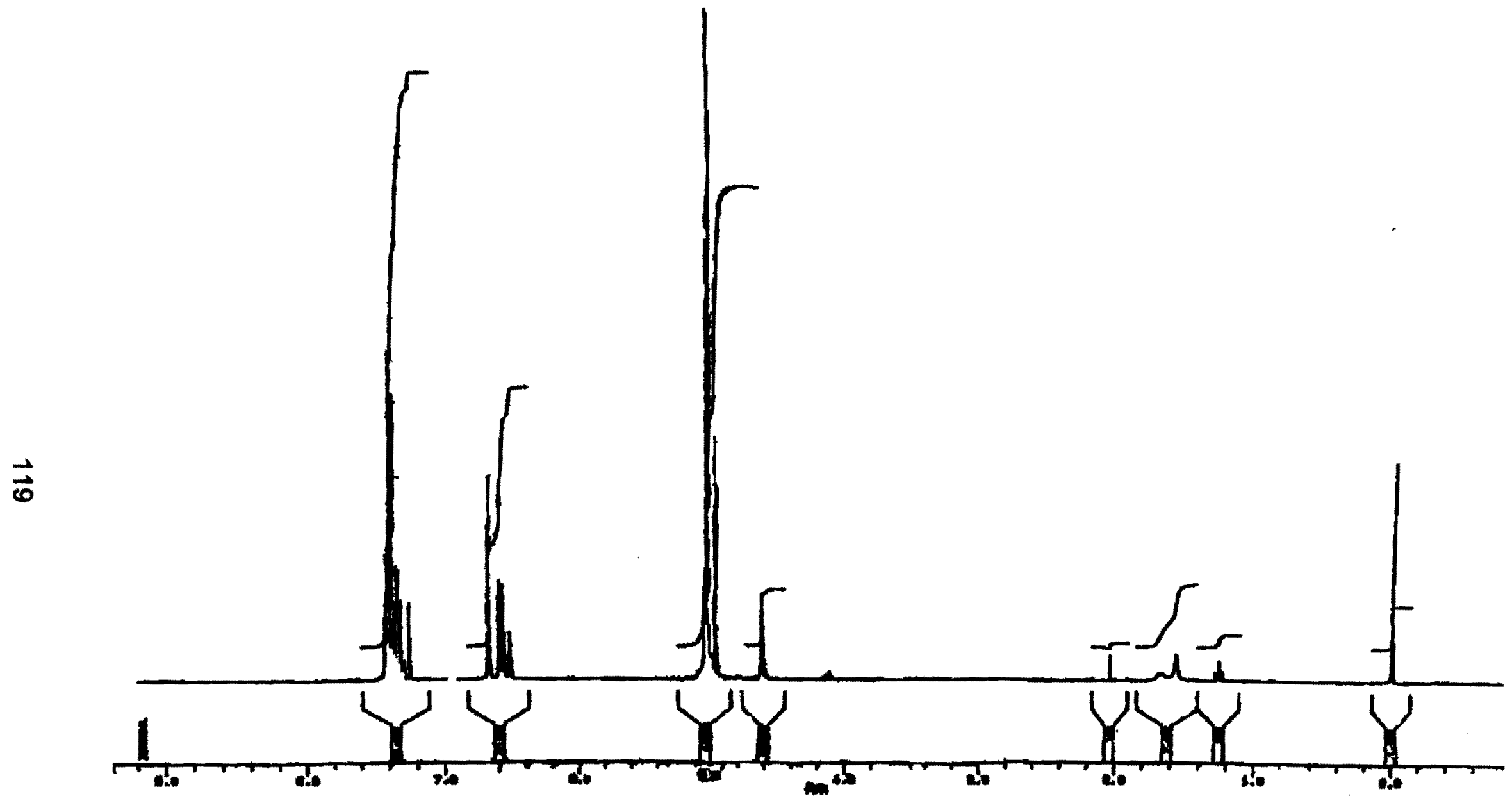

Figure 4-18. 300-MHz ${ }^{1} \mathrm{H}$ NMR spectrum of 3, 5-(3', 5'-benzyloxybenzyloxy)benzyl 3-(triethoxysilyl)propyl carbamate (10), G2 carbamate. 
$\vec{N}$

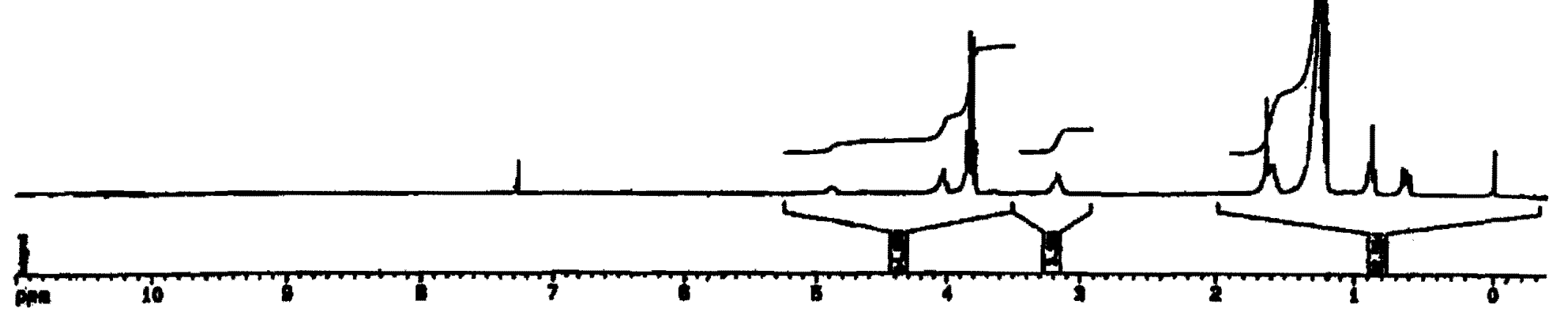

Figure 4-19. 300-MHz ${ }^{1} \mathrm{H}$ NMR spectrum of 1-octadecyl 3-(triethoxysilyl)propyl carbamate (11), stearyl carbamate. 







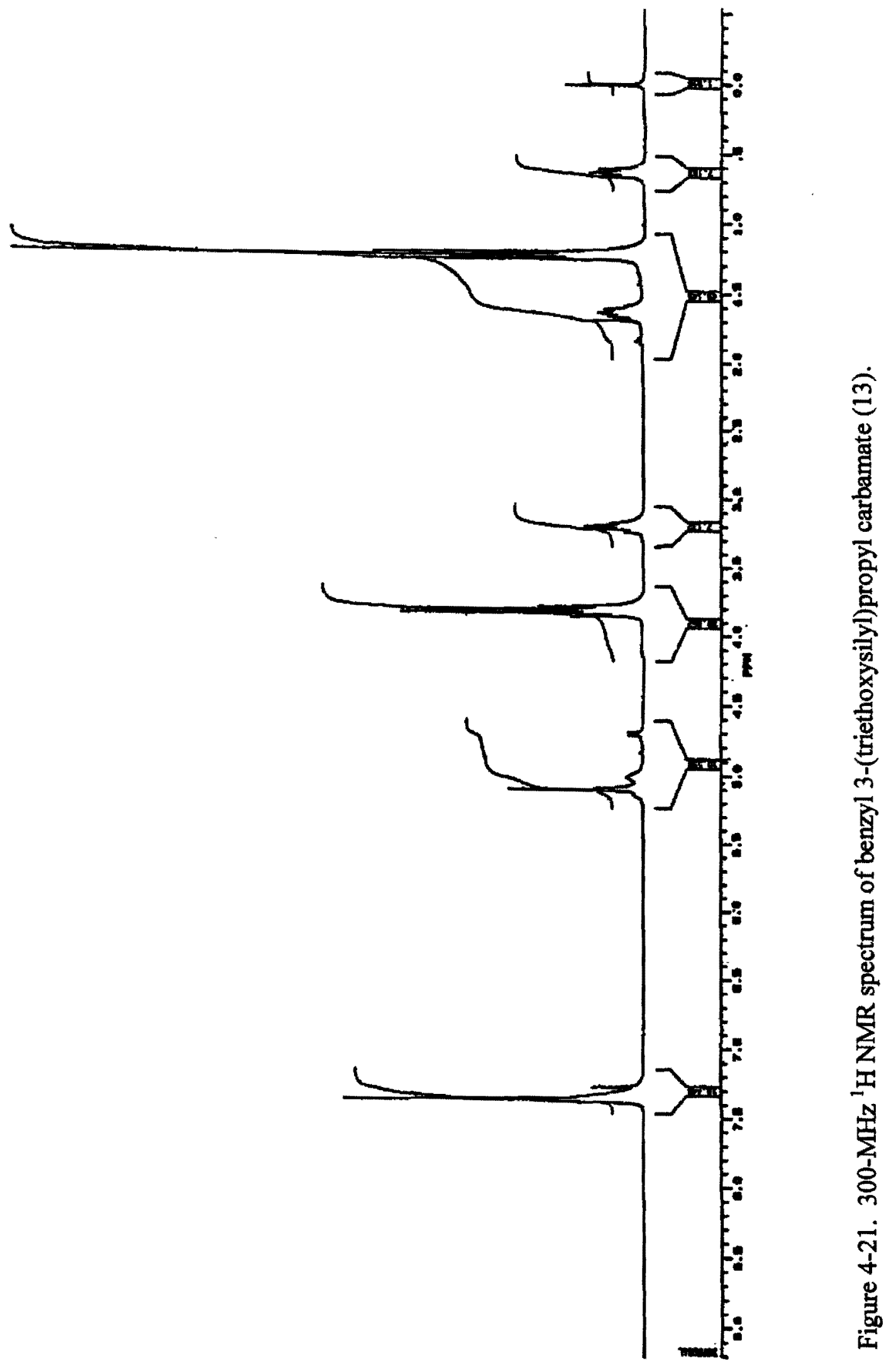




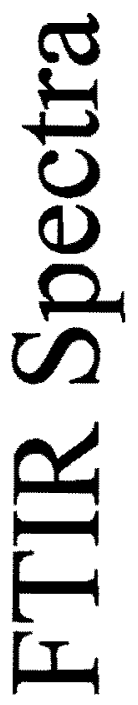




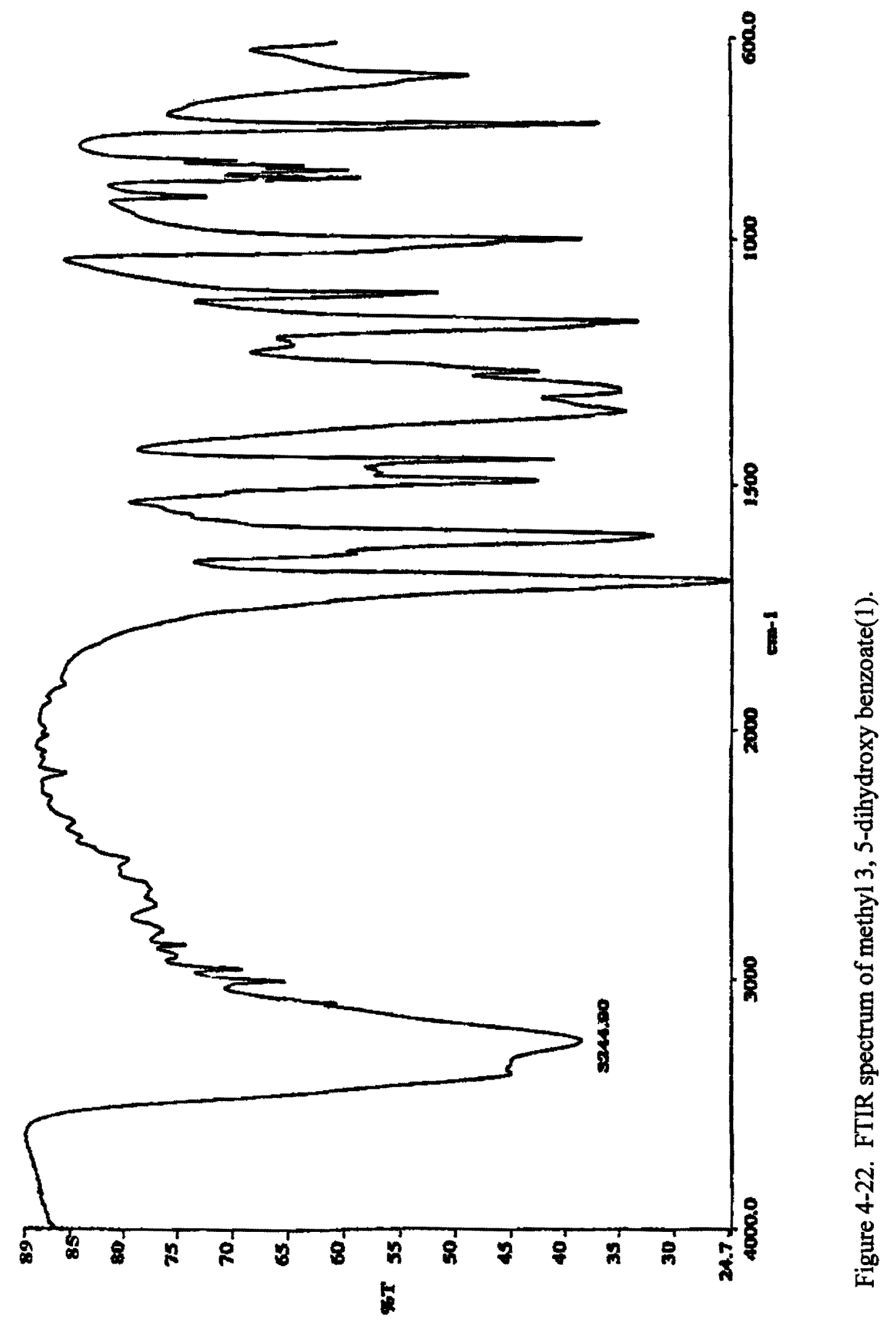




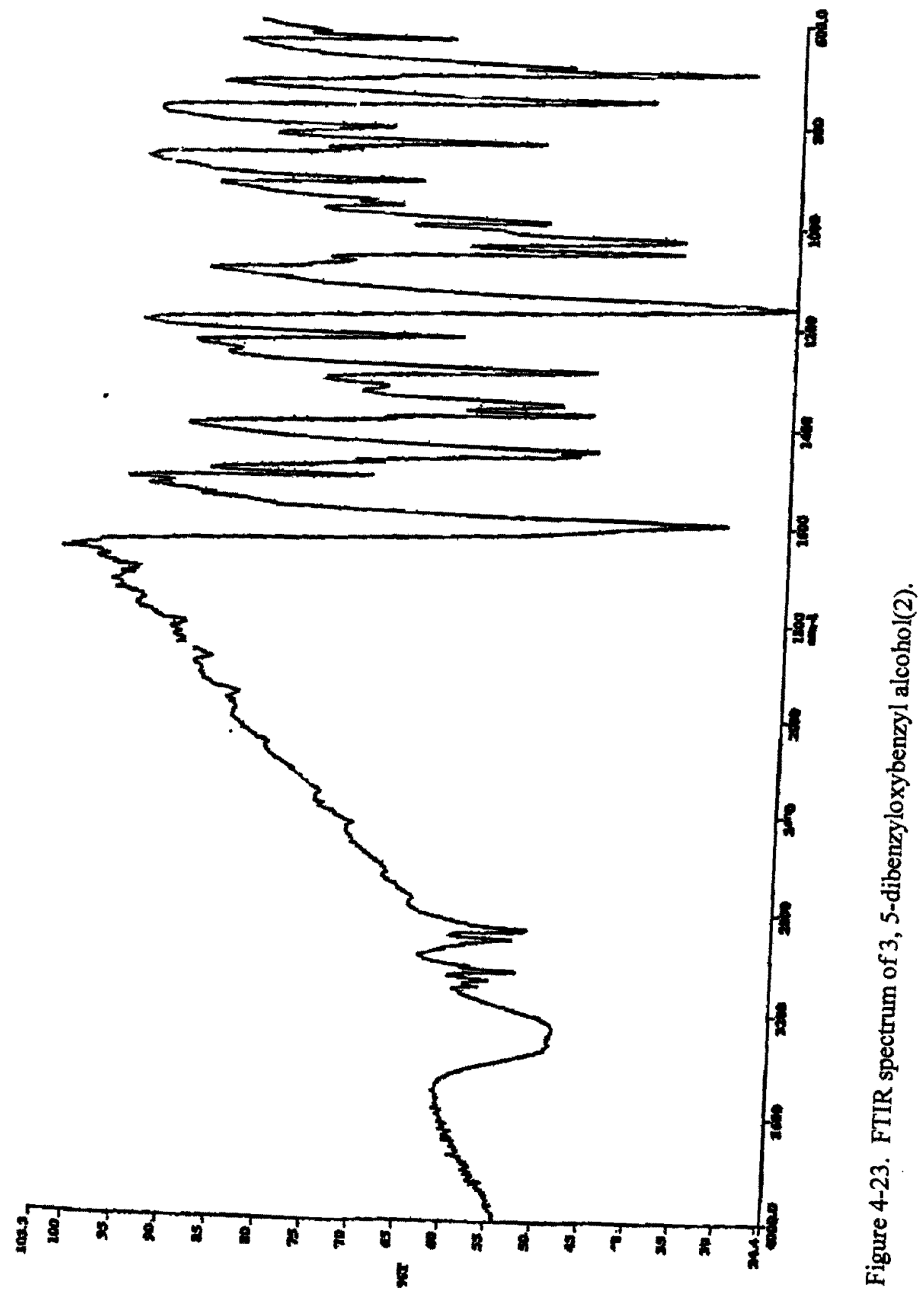






Figure 4-24. FTIR spectrum of 3, 5-dibenzyloxybenzyl methane sulphonate (3). 


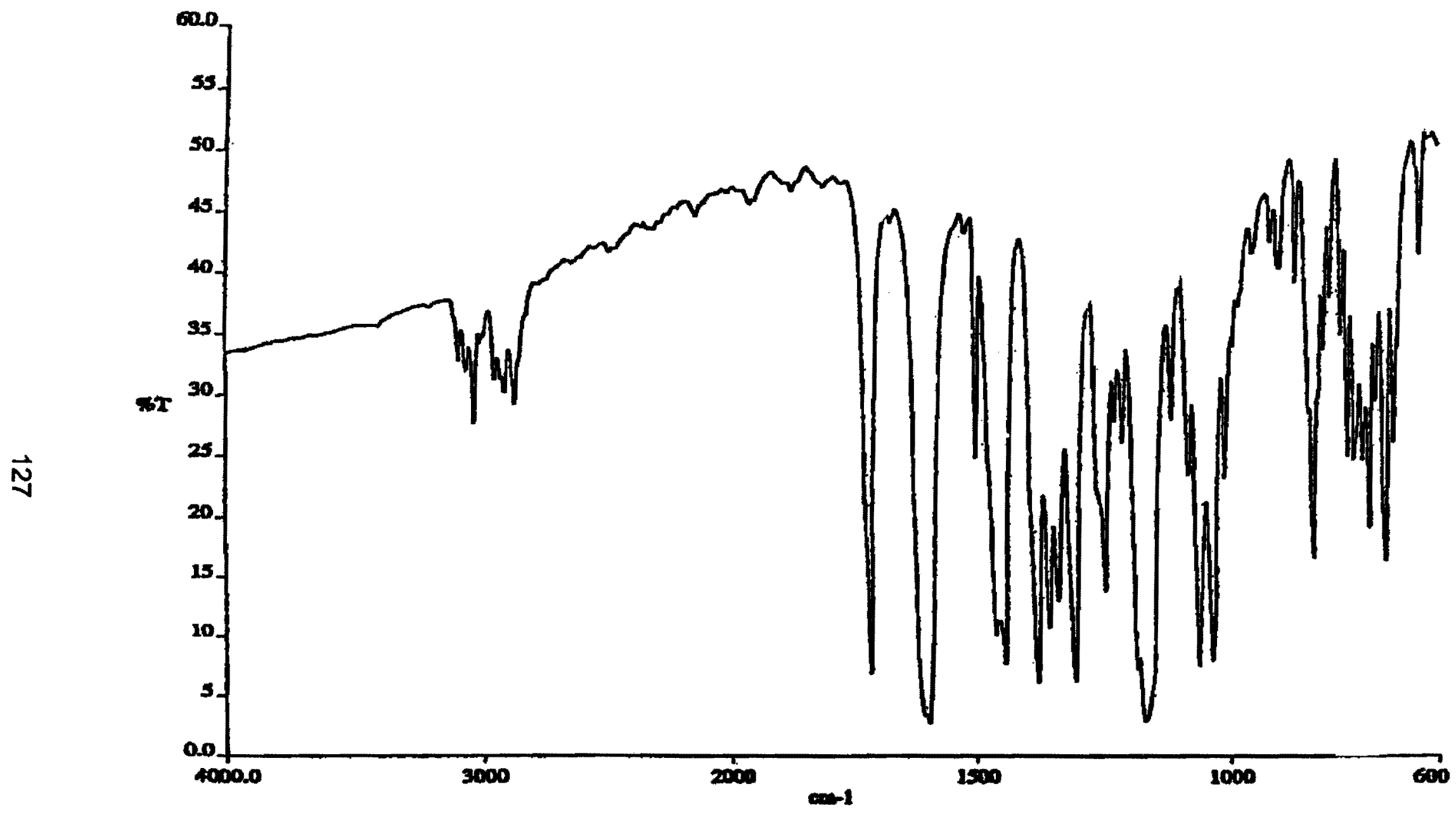

Figure 4-25. FTIR spectrum of methyl 3, 5-(3', 5'-benzyloxybenzyloxy) benzoate (4). 


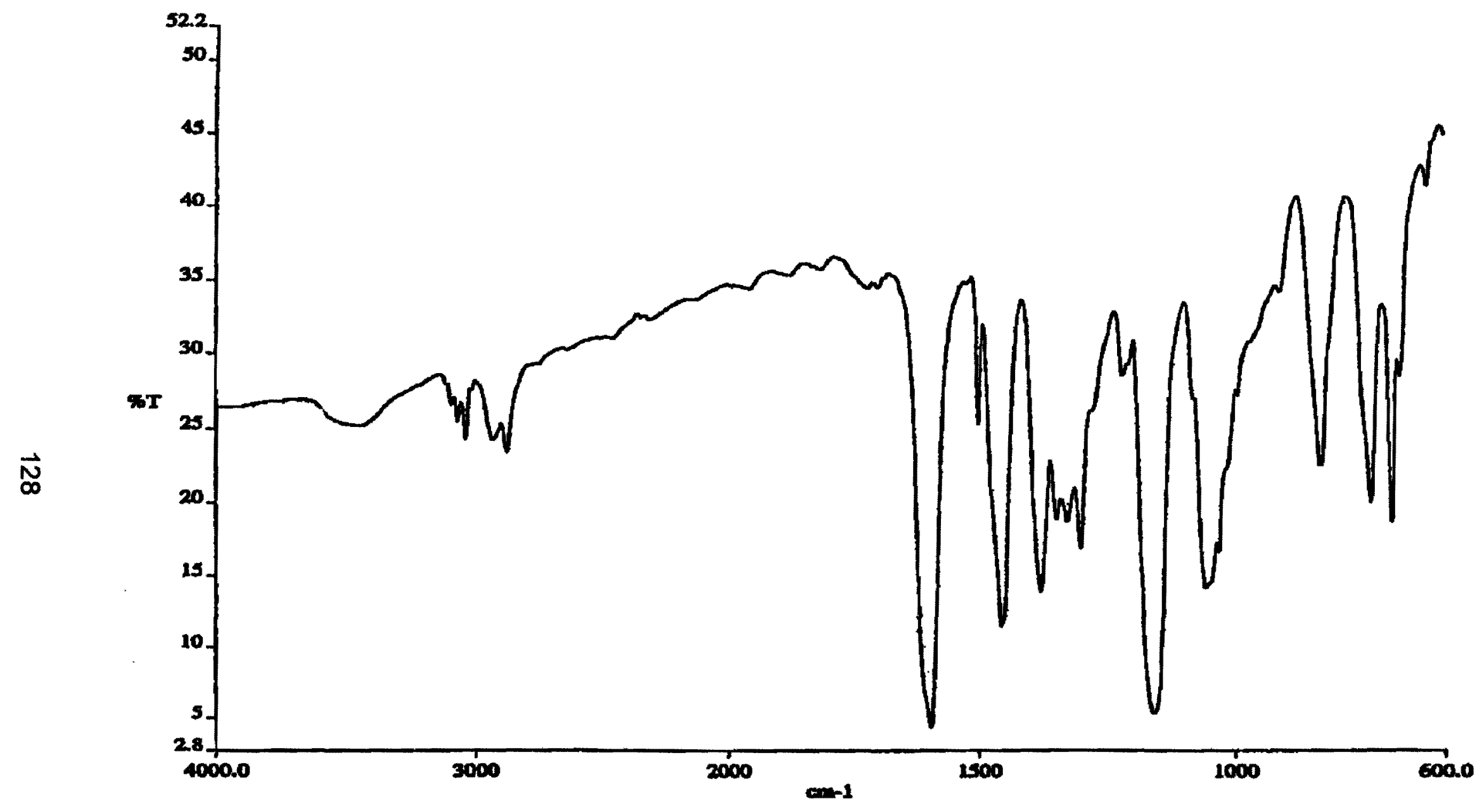

Figure 4-26. FTIR spectrum of 3, 5-(3', 5'-benzyloxybenzyloxy)benzyl alcohol (5). 


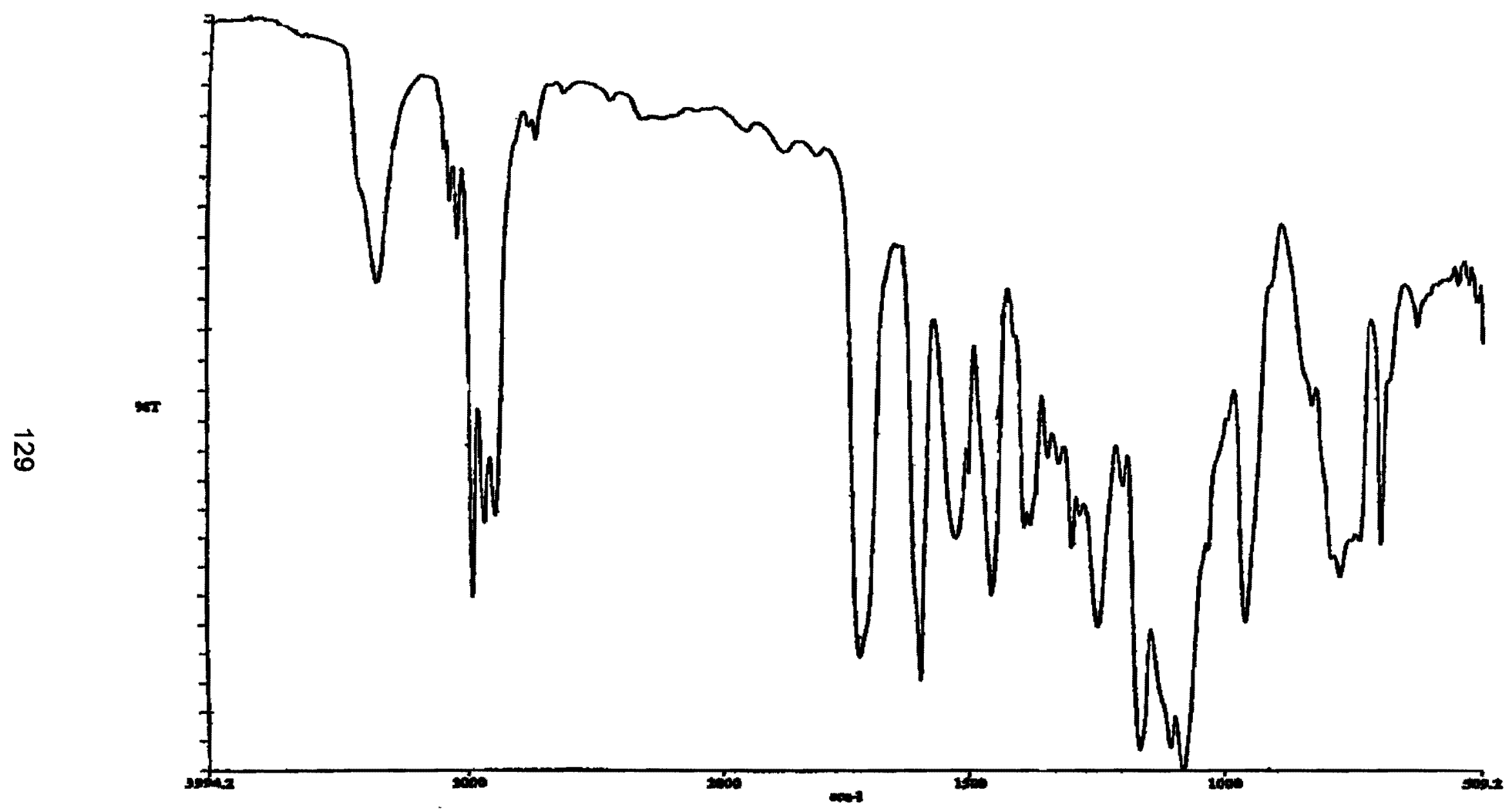

Figure 4-27. FTIR spectrum of 3, 5-dibenzyloxybenzyl 3-(triethoxysilyl)propyl carbamate (9), G1 carbamate. 


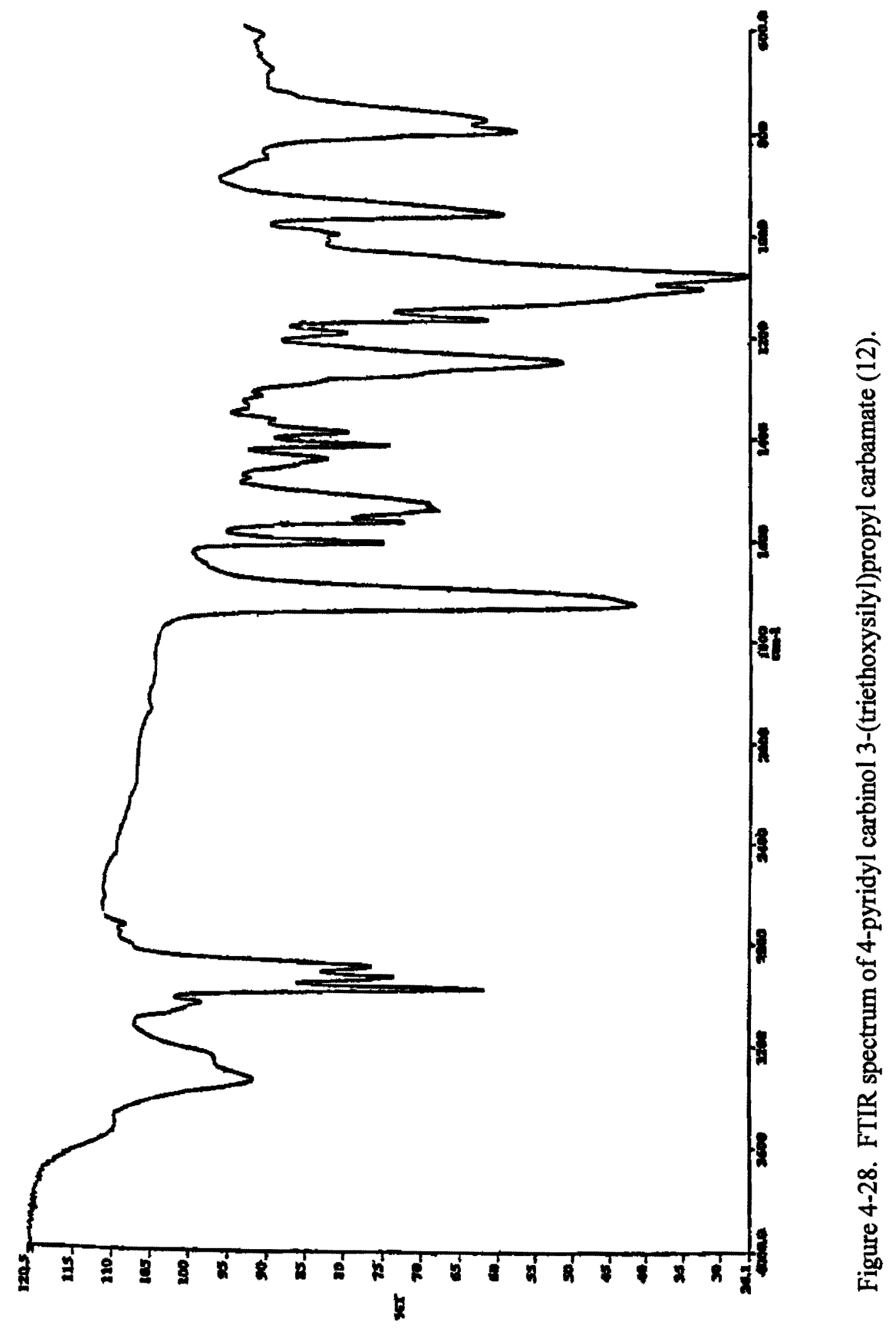




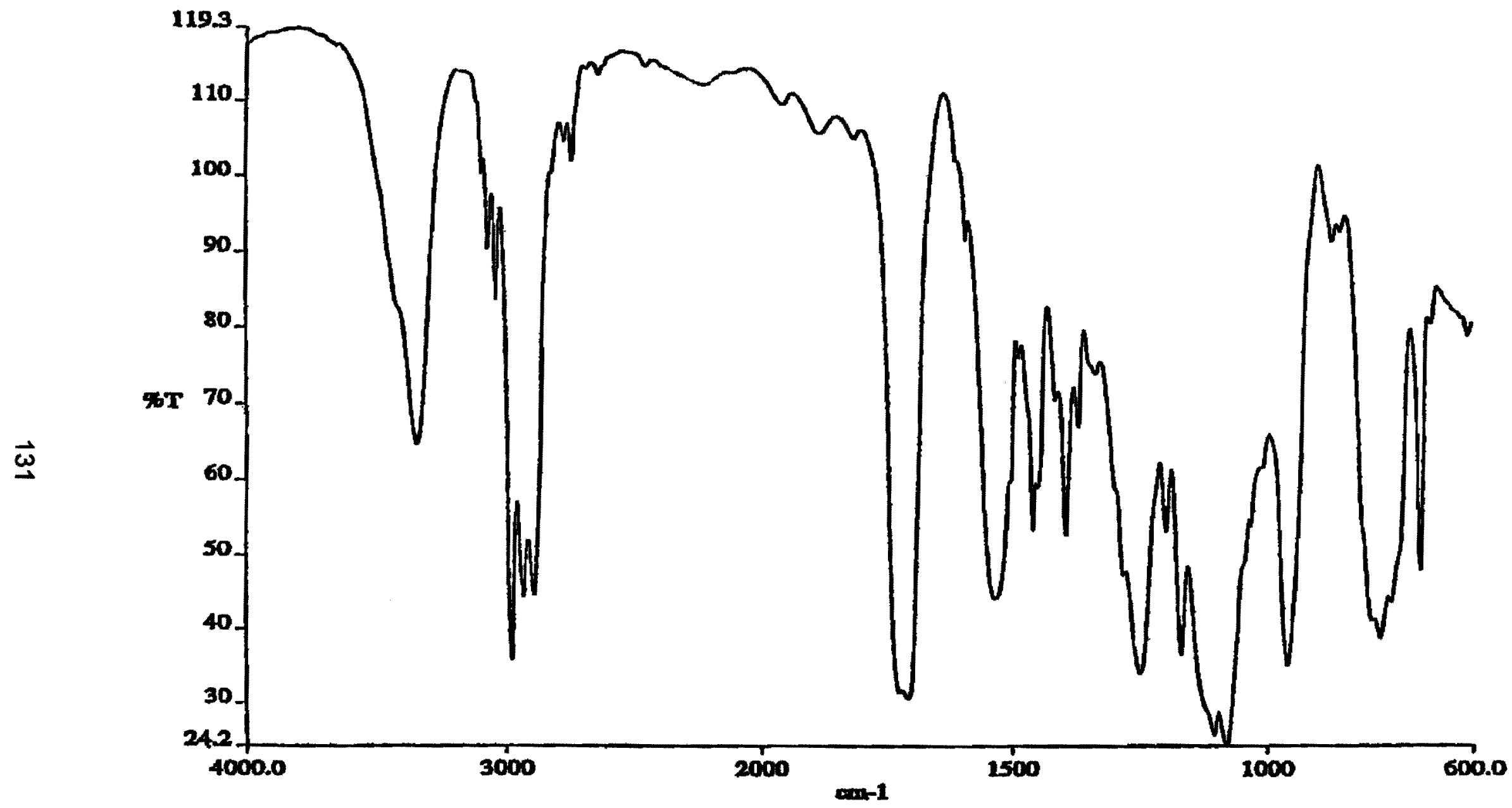

Figure 4-29. FTIR spectrum of benzyl 3-(triethoxysilyl)propyl carbamate (13). 


\subsection{References for Chapter 4}

1) Frechet, J. M. J.; and Hawker, C. J. J. Chem. Soc. Chem. Commun. 1990, 10101013.

2) Riley, J. M., Ph. D. Dissertation, Seton Hall University 1998.

3) Hanlon, P. M., Ph. D. Dissertation, Seton Hall University 1998.

4) Fieser, L. F.; Williamson, K. L. .

5) Hanson, J. E.; Reichmanis, E.; Houlihan, F. M.; Neenan, T. X. Chem. Mater. $1992,4,837$.

6) Hofle, G.; Steglich, W.; Vorbruggen, H. Agnew. Chem. Int. Ed. Engl. 1978, 17.

7) Van Dort, P. C.; Fuchs, P. L. J. Am. Chem. Soc. 1994, 116, 5657.

8) Tyler, T. L. Ph. D. Dissertation, Seton Hall University 1995. 


\section{CHAPTER 5}

\section{Dendrimer Coated Capillary Columns in OT-CEC}

\subsection{Introduction}

The most demanding challenge in CEC research is the preparation of coated capillary columns which are stable and reproducible. The investigation of polymeric materials as the stationary phase in CEC has just begun in the past few years. Stationary phases can be bonded onto the inner walls of fused silica capillaries in the OT-CEC format, or the phases can be bonded to particles packed into a capillary column. Most research in CEC has focused on the preparation and operation of packed capillary columns (PCC) using commercially available packing materials (silica particles modified with $\mathrm{C}_{18}$ stationary phase). ${ }^{1-5}$ Only a few publications have addressed the importance and difficulties in preparing stationary phases on the inner walls of fused silica capillaries for OT-CEC.6-8 Since the separation in OT-CEC is based on partitioning of the solutes between the mobile phase and stationary phase, the chemistry of the stationary phases and the preparation of stationary phases are critically important to the development of this technique. In this research, the author explored an interesting new class of polymers, poly(aryl ether) dendrimers, as the stationary phase in OT-CEC. Their unusual chemical and physical properties have great potential in many applications. Polyamidoamine starburst dendrimers (PAMAM-SBDs) (Figure 5-1) have shown promising results as a pseudo-stationary phase in Micellar Electrokinetic Capillary Chromatography (MECC). It is the author's desire to generate a public interest in exploiting the potential of dendritic polymers as stationary phases in chromatographic separations. 
This research focuses on the investigation of non-conventional polymers, poly(aryl ether) dendrimers (Figure 5-2), as a stationary phase in an open tubular format. This chapter details the experimental work and the OT-CEC results.



Figure 5-1. The structure formula of PAMAM, $4^{\text {th }}$ generation. 9 


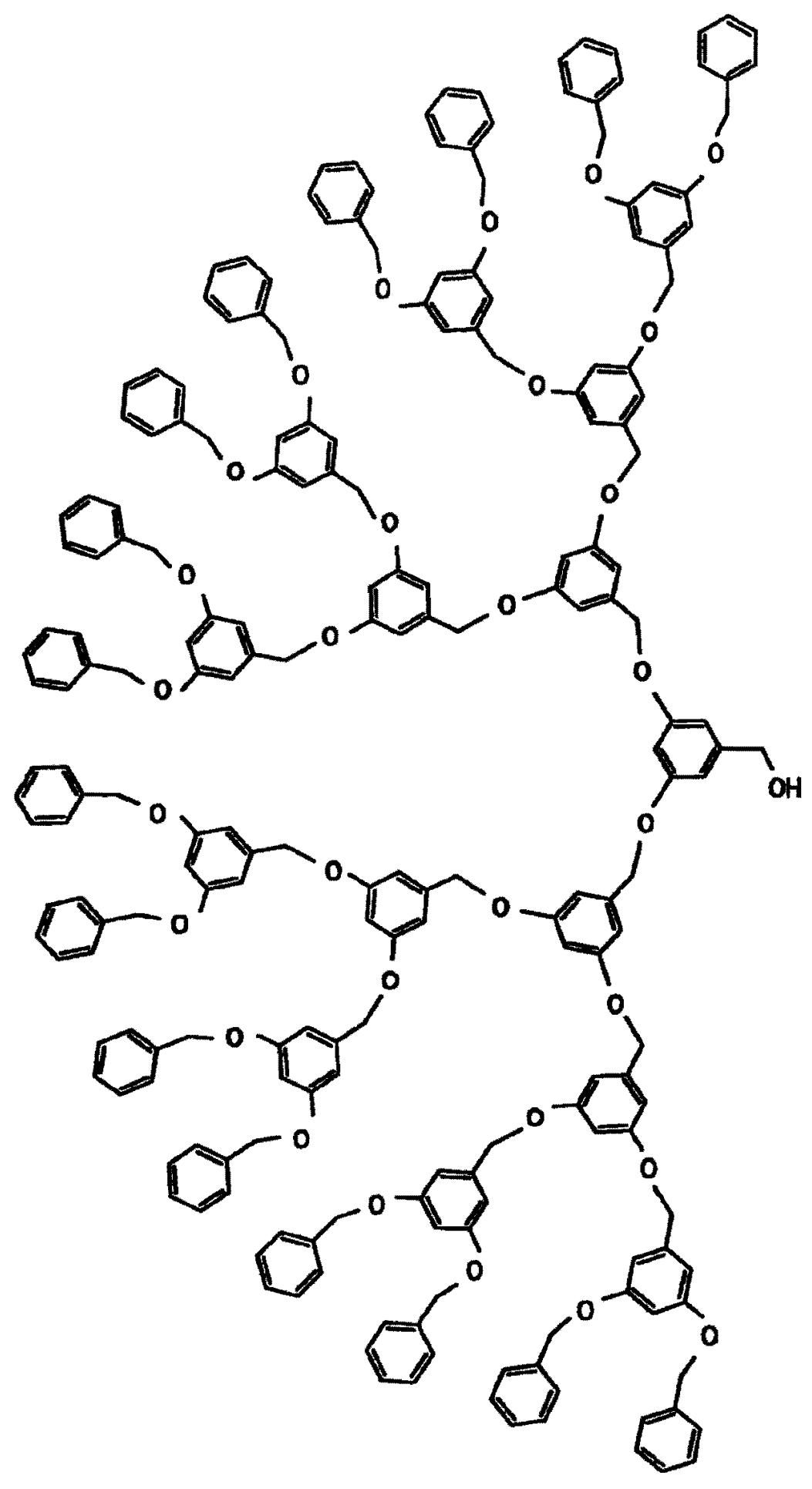

Figure 5-2. The structural formula of a poly(aryl ether) dendrimer, $4^{\text {th }}$ generation. 9 


\subsection{Open tubular columns (OTCs)}

Using open tubular columns is the simplest approach to electrically driven flow. OTCs are capillary columns with an inner diameter ranging between 10 to $50 \mu \mathrm{m}$ with the stationary phase located on its inner wall as a very thin film. Several important reports on the use of OTCs in CEC were published in the last five years. ${ }^{10,11}$ The most common approach for attaching the stationary phase to the surface of the capillary is through silane chemistry, using surface silanols. However, it is very difficult to prepare OTCs with very narrow capillaries $(<15 \mu \mathrm{m}$ i.d.) using appropriate coating materials of sufficient retentive and mass loadability characteristics. This is because the inner walls of the fused silica capillaries are relatively smooth with little surface area and a limited number of available silanols resulting in lower phase ratios. It has been shown that the phase ratio values of OTC are approximately 350 times smaller than those of commercial LC packings. ${ }^{12,13}$ With such conditions, the capacity factor for most of the solutes becomes so small that OTCs cannot be used for analytical purposes. In addition, the small diameter introduces practical problems associated with detection.

Many efforts have aimed at improving the surface area and reactivity of the inner walls of the fused silica capillaries. Early procedures consisted of etching the internal surface, then the stationary phase was chemically bonded directly onto the treated inner wall of the capillary. ${ }^{14}$ This type of column provided very low sample capacity. Another approach involved cross-linking of polymeric stationary phases. Polysiloxanes $15-17$ and polyacrylates 18,19 were applied to the inner walls. Columns prepared by this procedure gave good stability, high phase ratio, and adequate analyte

retention. However, these columns have shown a total suppression in EOF. 20-22 
Therefore, neutral compounds and anions remained at the anode end of the capillary indefinitely, as discussed in the Section 2.2.3. Yeung et al addressed these problems by using a cation surfactant as an additive in the mobile phase to generate an EOF in these columns, and thus separate neutral compounds in reversed-phase mode. ${ }^{23}$ Schurig and Mayer employed the same technique to prepare a chiral column using a chiral polymeric material (Chirasil-Dex) to separate enantiomers. ${ }^{24}$ However, they found the column efficiency was poor, due to low diffusion coefficients in the cross-linked phase.

It is known that porous materials provide better diffusion. The approach of using such materials as adsorbent layers in capillaries was first explored by Tock et al. ${ }^{25}$ Their procedure involved a two step process: (1) applying a thin porous silica layer with large surface area on the inner wall of the capillary (2) attaching functional groups onto the prepared layer through chemical bonding. However, the thickness of the layer obtained by dynamic coating was small and its porosity was so low that it was unable to bond a substantial amount of alkylsilane. Crego et al 26 further refined this method by preparing a thin layer of silica gel with chemically bonded ODS on the internal wall of fused silica capillaries. The preparation of the silica layer was based on the hydrolytic polycondensation and gelling of tetraethoxysilane (TEOS) in a pH-fixed water-ethanol solution. 27 This procedure showed promising results and the reproducibility of the method in terms of $\mathcal{X}^{\prime}$ and reduced plate height from the column was quite good.

Recently, Guo and Colón 28,29 explored sol-gel technology to produce porous silica glass films that can be cast onto the inner walls of OTCs for LC and CEC applications. The surface area of the inner wall of fused silica capillaries after this treatment is significantly increased. The porous glass coating was found to be stable in 
acidic and basic conditions. At the present time, the fabrication of a column requires skill and experience, and many consider it an art.

\section{$5.3 \quad$ Experimental}

5.3.1 Materials. Fused silica capillary tubing of $50 \mu \mathrm{m}$ i.d. was purchased from Polymicro Technologies. Column coating materials were synthesized at Seton Hall University research laboratory, Department of Chemistry. All chemicals used in the syntheses were purchased from Aldrich and were used without further purification (see

Chapter 4, Section 4.2.1). All buffer solutions, protein test mixture (consisting of lysozyme, cytochrome $\mathrm{C}$ and ribonuclease $\mathrm{A}$ ) and anion test mixture (consisting phydroxyphenylacetic acid, p-hydroxybenzoic acid and benzoic acid) were from Beckman Coulter. The following compounds: benzyl bromide, benzyl triethoxysilane, sodium hydroxide, potassium hydroxide, p-toluic acid, phenylacetic acid, p-chlorobenzoic acid, naphthalene, phenanthrene, pyrene, aniline, o-methylaniline, m-nitroaniline, toluene, tetrahydrofuran (THF), 3-aminopropyl triethoxysilane and 3-(triethoxysilyl)propyl isocyanate were purchased from Aldrich Chemical Company. HPLC grade acetonitrile and methanol were from Burdick and Jackson. Deionized water $\left(\mathrm{ddH}_{2} \mathrm{O}\right)$ was obtained from a Milli-Q water system.

\subsubsection{Column coatings}

As mentioned in Section 5.2, the coating of the stationary phase inside the fused silica capillaries is complicated by two factors. First, the small inner diameters: the small dimensions of the capillary make it very difficult to implement modification procedures 
for stationary phase preparation. Second, the smooth surface area: the number of surface silanol groups available for chemical reactions is very limited. This leads to a low phase ratio for the stationary phase after chemical modification. In an attempt to minimize undesired effects, the author adopted the alkali etch procedure to increase the surface area as the first step of column preparation. Once the surface is prepared, a second step is needed to attach the stationary phase onto the silica substrate through chemical bonding.

The first part of this investigation was to bond the dendrimers via the $\mathrm{Si}-\mathrm{O}-\mathrm{C}$ bond or $\mathrm{Si}-\mathrm{O}-\mathrm{Si}$ bond and to determine the stability and reproducibility of the two linkage types. Initially, we investigated an $\mathrm{Si}-\mathrm{O}-\mathrm{C}$ monodentate linkage using benzyl bromide (G0 bromide) and poly(aryl ether) dendrimers as the bromide forms as column modifiers. Figure 5-3 and Figure 5-4 depict the $\mathrm{Si}-\mathrm{O}-\mathrm{C}$ bonding type. The $\mathrm{Si}-\mathrm{O}-\mathrm{Si}$ tridentate linkages were studied using benzyl triethoxysilane as the zero generation of poly(aryl ether) dendrimers (Figure 5-5). The capillary columns were prepared by the dynamic coating procedure.

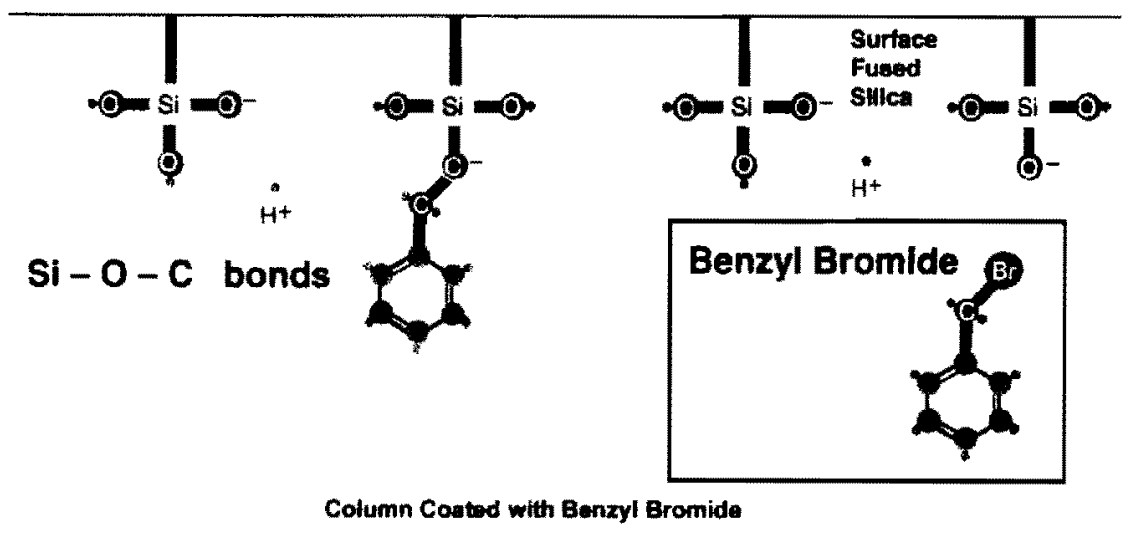

Figure 5-3. $\mathrm{Si}-\mathrm{O}-\mathrm{C}$ monodentate bond with $\mathrm{G} 0$ from the bromide. 


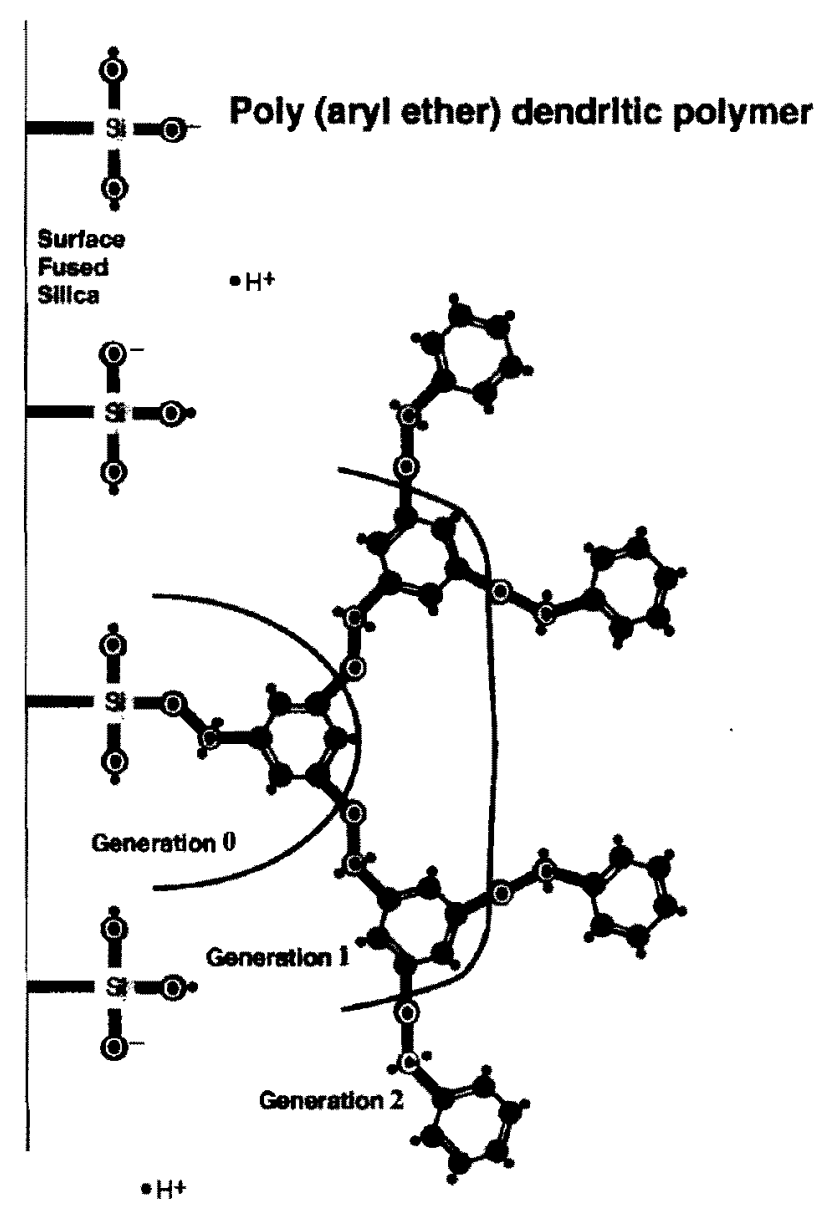

Figure 5-4. Si-O-C monodentate bond of poly(aryl ether) dendrimer.

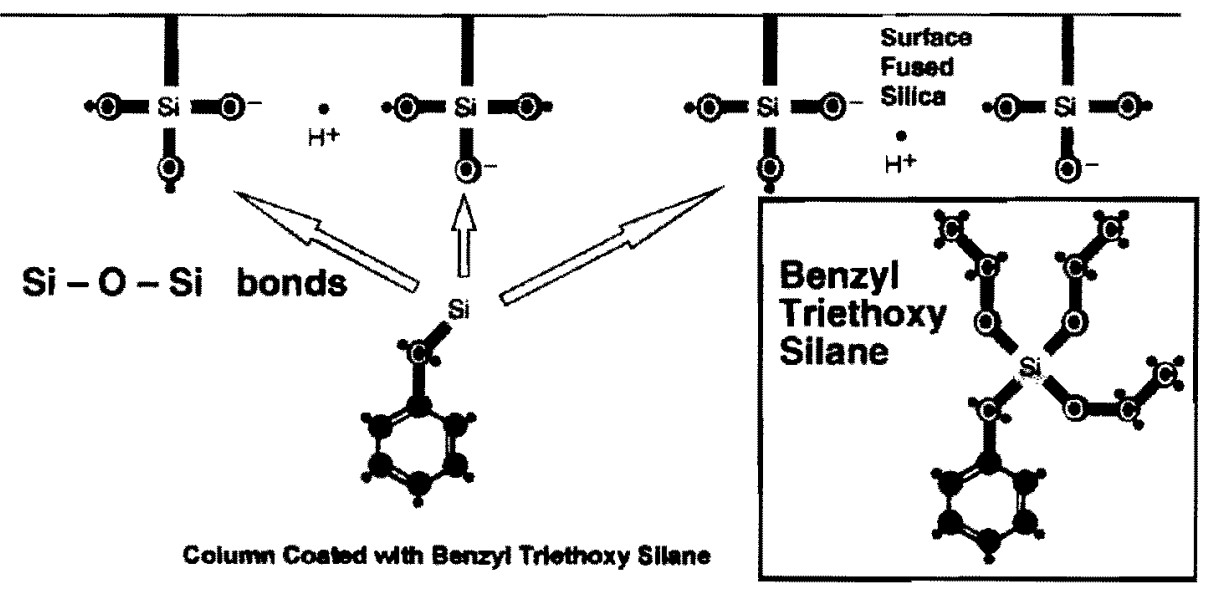

Figure 5-5. Illustrates the $\mathrm{Si}-\mathrm{O}-\mathrm{Si}$ tridentate bonds. 


\subsubsection{Dynamic coating procedure}

This procedure is commonly used in CE protein analysis. The ionizable siloxane surface of a capillary tube was prepared by flushing the tube with $1 \mathrm{~N} \mathrm{NaOH}$ solution using 20 psi for 10 minutes. The capillary was then rinsed with the THF solution containing $0.5 \%$ of dendrimer ( 10 minutes), followed by water (10 minutes), and finally the run buffer (10 minutes). This washing sequence was repeated prior to each sample analysis. Samples were injected by pressure injection for 5 seconds. Separation was carried out at $30 \mathrm{kV}$, with UV detection at $254 \mathrm{~nm}$ or $280 \mathrm{~nm}$ depending on the analytes. The temperature of the column was maintained at $20^{\circ} \mathrm{C}$.

\subsubsection{Effects of dendritic polymers coating}

The effect of dendritic polymers of different size on the peak resolution and the migration time of the analytes was investigated. These preliminary experiments were performed using a commercially available test mixture (benzoic acid, p-hydroxybenzoic acid, and p-hydroxyphenylacetic acid) to test the performance of the dendrimer coated column. The resulting electropherograms showed that the electroosmotic flow was suppressed when the G0 and Gl poly(aryl ether) dendrimer coated columns were compared to an uncoated column and for some applications the resolution improved using a dendrimer coated column (Figure 5-6). The calculated apparent mobility, $\mu_{\text {apps, }}$ from the migration times observed in the experiment are listed in Table 5-1. The migration time increases with increasing size of the dendrimer (Figures 5-3 and 5-4, Table 5-1). The EOF was reduced approximately 1.6 to 2 fold (Table 5-1). 
<smiles>O=C(O)c1ccccc1</smiles>

Benzoic acid<smiles>O=C(O)c1ccc(O)cc1</smiles>

p-Hydroxybenzoic acid<smiles>CC(=O)Cc1ccc(O)cc1</smiles>

p-Hydroxyphenyl acetic acid
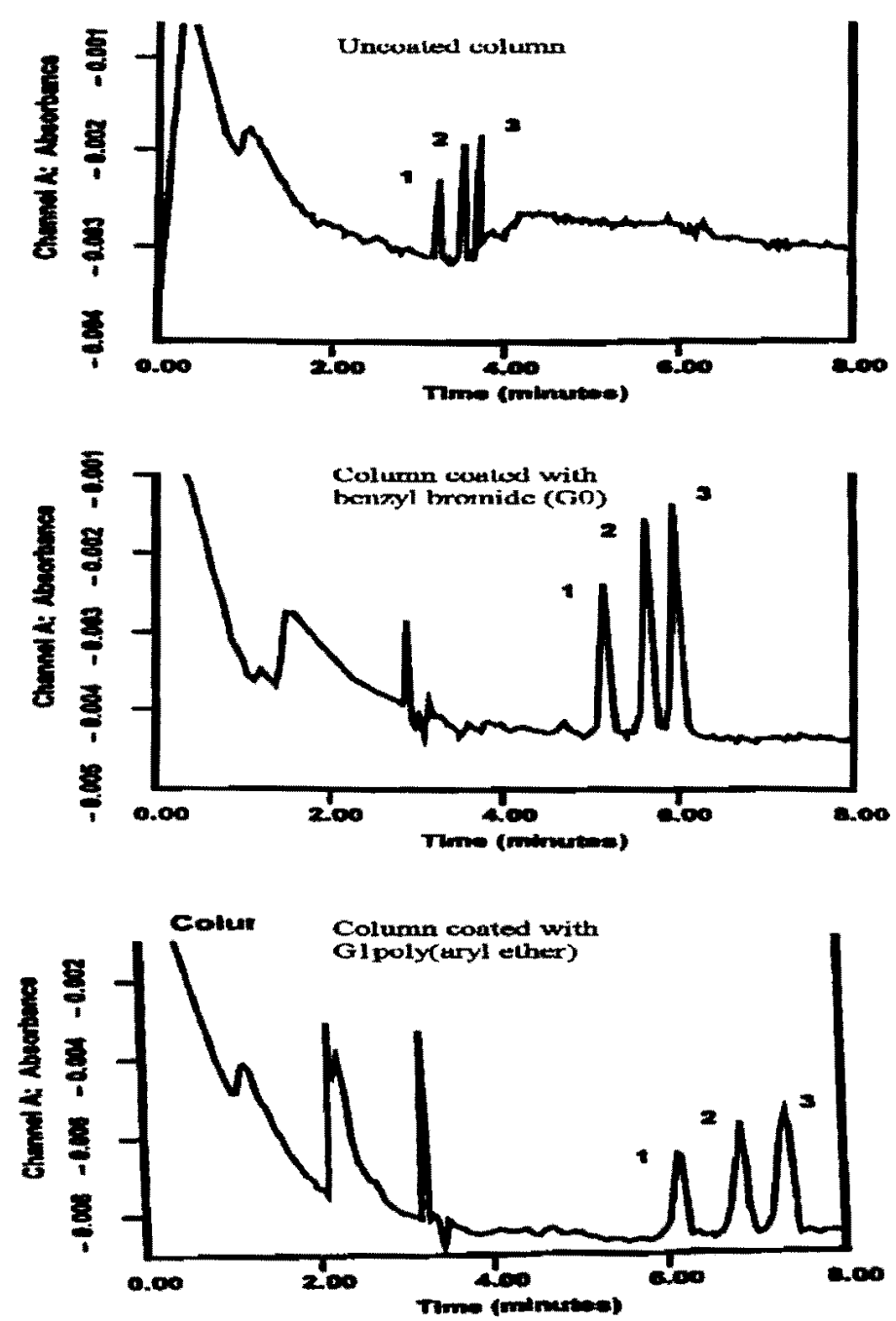

Figure 5-6. Anion test mixture separation comparison with a uncoated column, benzyl bromide coated column, and a G1 Poly(aryl ether) coated column: (1) Benzoic acid, (2) p-Hydroxybenzoic acid and (3) p-Hydroxyphenylacetic acid. 
Table 5-1. Summary of apparent mobility observed with dynamic coating process.

\begin{tabular}{|c|c|c|c|c|}
\hline Analytes & $\begin{array}{c}\text { Migration time } \\
\mathrm{t}_{\mathrm{r}} \text { in min. }\end{array}$ & $\begin{array}{c}\text { apparent mobility } \\
\mu_{\text {app }} \text { in } \mathrm{cm}^{2} / \mathrm{v} \cdot \mathrm{s}\end{array}$ & $\begin{array}{c}\text { Ratio of } \mu_{\text {app }} \\
\text { (estimated) }\end{array}$ & Coating materials \\
\hline $\begin{array}{c}\text { p-hydroxy phenylacetic } \\
\text { acid }\end{array}$ & 3.28 & $4.30 \times 10$ & 1.00 & Uncoated \\
& 5.28 & $2.67 \times 10$ & 1.61 & G0 coated \\
& 6.24 & $2.27 \times 10$ & 1.89 & G1 coated \\
& 5.44 & $2.60 \times 10$ & 1.65 & Bn triethoxy silane coated \\
\hline p-hydroxybenzoic acid & 3.52 & $4.00 \times 10$ & 1.00 & Uncoated \\
& 5.76 & $2.45 \times 10$ & 1.63 & G0 coated \\
& 6.88 & $2.05 \times 10$ & 1.95 & Gl coated \\
& 6.24 & $2.27 \times 10$ & 1.76 & Bn triethoxy silane coated \\
\hline Benzoic acid & 3.68 & $3.83 \times 10$ & 1.00 & Uncoated \\
& 6.08 & $2.31 \times 10$ & 1.66 & G0 coated \\
& 7.36 & $1.92 \times 10$ & 1.99 & G1 coated \\
& 6.72 & $2.10 \times 10$ & 1.82 & Bn triethoxy silane coated \\
\hline
\end{tabular}

\subsubsection{Effects of dendritic polymers on analytes}

The effect of dendritic polymers on the analytes was also studied. Three different categories of analytes were used to evaluate the column efficiency, resolution, and selectivity. The analytes used in the experiments are: (1) anions: p-toluic acid, phenylacetic acid, and p-chlorobenzoic acid, (2) neutral compounds: naphthalene, phenanthrene, and pyrene, (3) cations: aniline, o-methylaniline, and m-nitroaniline. Improved efficiency, resolution and selectivity were observed for both the anions and the polyaromatic hydrocarbon mixtures when the capillary was coated with poly(aryl ether) dendrimer (G1). Figure 5-7 shows the anion electropherograms, which were obtained with $\mathrm{pH} 9.2,5 \mathrm{mM}$ borate buffer and $30 \%$ methanol. Figure 5-8 shows the polyaromatic 
hydrocarbon electropherogram, which was obtained with $\mathrm{pH} 9.2,10 \mathrm{mM}$ borate buffer and 30\% methanol. The cation electrograms did not exhibit the desired resolution when the modified columns were used.

In general, we have found dendrimer coated capillary columns using the $\mathrm{Si}-\mathrm{O}-\mathrm{C}$ linkage show improved resolution and increased efficiency for $\mathrm{CE}$ applications. However, the coating sequence must be repeated prior to each sample analysis to give better reproducibility. To improve column stability and reproducibility, other bonding mechanisms and preparation methods were explored vide infra.

\subsubsection{Effects of $\mathrm{Si}-\mathrm{O}-\mathrm{C}$ versus $\mathrm{Si}-\mathrm{O}-\mathrm{Si}$ bonds}

The Si-O-Si bonding mechanism was investigated with benzyl triethoxysilane as a model for column performance evaluation. We believe that the formation of a bidentate or tridentate $\mathrm{Si}-\mathrm{O}-\mathrm{Si}$ attachment can be produced under the recommended conditions, which results in a more stable bonded stationary phase inside the capillary, compared to the benzyl bromide in which only a single $\mathrm{Si}-\mathrm{O}-\mathrm{C}$ bond can be formed. The effect of $\mathrm{Si}-\mathrm{O}-\mathrm{Si}$ bonds versus $\mathrm{Si}-\mathrm{O}-\mathrm{C}$ bonds was examined by comparing the electropherograms obtained with the benzyl triethoxysilane and the benzyl bromide coated capillary columns. We found better column efficiency, resolution, and stability with the $\mathrm{Si}-\mathrm{O}-\mathrm{Si}$ bonds when the test mixture of p-hydroxyphenylacetic acid, p-hydroxybenzoic acid, and benzoic acid was injected on a capillary column that was pretreated with benzyl triethoxysilane. A diagram of the $\mathrm{Si}-\mathrm{O}-\mathrm{Si}$ tridentate bonding and $\mathrm{Si}-\mathrm{O}-\mathrm{C}$ monodentate bonding is illustrated in Figures 5-5 and Figures 5-3, respectively. A resolution comparison between the $\mathrm{Si}-\mathrm{O}-\mathrm{Si}$ versus $\mathrm{Si}-\mathrm{O}-\mathrm{C}$ bonding can be found in Table 5-2. 
The anion test mixture electropherograms, Figure 5-9, demonstrate improved resolution and efficiency for $\mathrm{Si}-\mathrm{O}-\mathrm{Si}$ bonding versus $\mathrm{Si}-\mathrm{O}-\mathrm{C}$ bonding.

Unlike the column modification process used with benzyl bromide, the procedure to form $\mathrm{Si-O}-\mathrm{Si}$ covalent bond was a multi-step process. A new method was developed to prepare the $\mathrm{Si}-\mathrm{O}-\mathrm{Si}$ bonded capillary column. This method was intended to produce a permanent modification to the inner surface of the fused capillary. Column curing at a high temperature is necessary to produce the desired thin film on the inside wall of the capillary. In this experiment, the capillary was etched first with $1 \mathrm{~N} \mathrm{NaOH}$ solution for one hour and then flushed with $1 \%$ benzyl triethoxysilane in toluene for one hour. The capillary containing the silane solution was placed in an oven with both ends sealed and was baked at $70^{\circ} \mathrm{C}$ for a period of 5 to 12 hours. After the curing process, the ends were cut and the column was flushed with toluene for one hour to remove excess coating material. The coated column was dried at 50 to $60^{\circ} \mathrm{C}$ for 2 hours with a gentle flow of Helium. Although improved resolution and stability were observed with columns coated by $\mathrm{Si}-\mathrm{O}-\mathrm{Si}$ bonds, in order to achieve optimal reproducibility, the capillary must be recoated after every five injections when this procedure is used.

The positive results for the Si-O-Si bonding experiments provided the impetus to focus our research in this direction. 


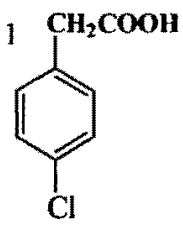

p-chlorophenyl acetic acid

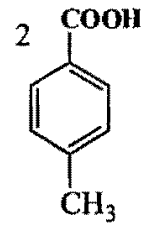

p-toluic acid

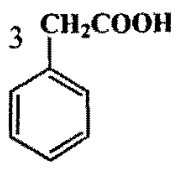

phenylacetic acid

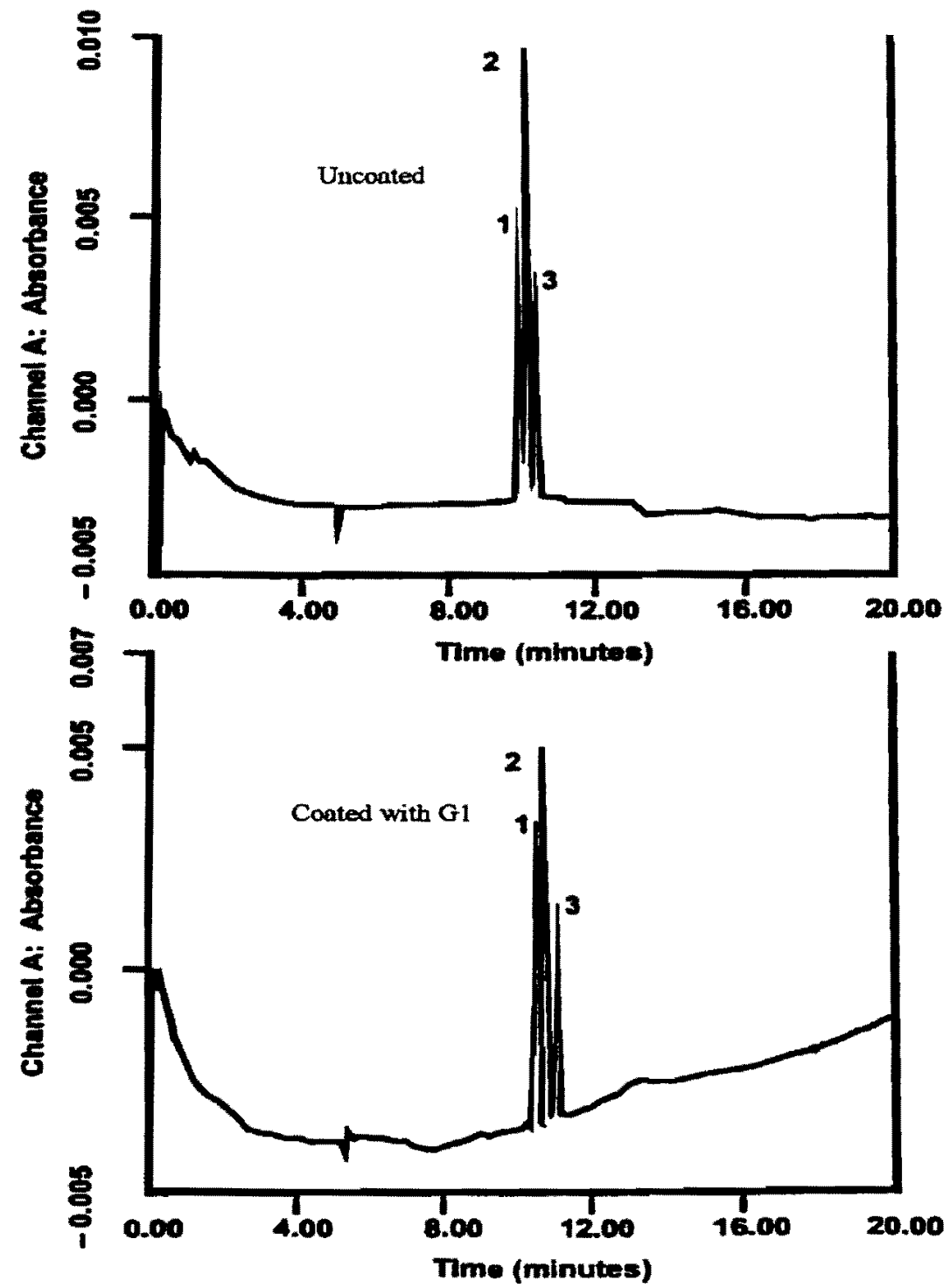

Figure 5-7. Anion electropherograms obtained with uncoated and G1 coated capillary columns: (1) p-chlorophenylacetic acid, (2) p-toluic acid and phenylacetic acid. 
<smiles>c1ccc2ccccc2c1</smiles><smiles>c1ccc2c(c1)ccc1ccccc12</smiles>

Naphthalene

Phenanthrene<smiles>c1cc2ccc3cccc4ccc(c1)c2c34</smiles>

pyrene

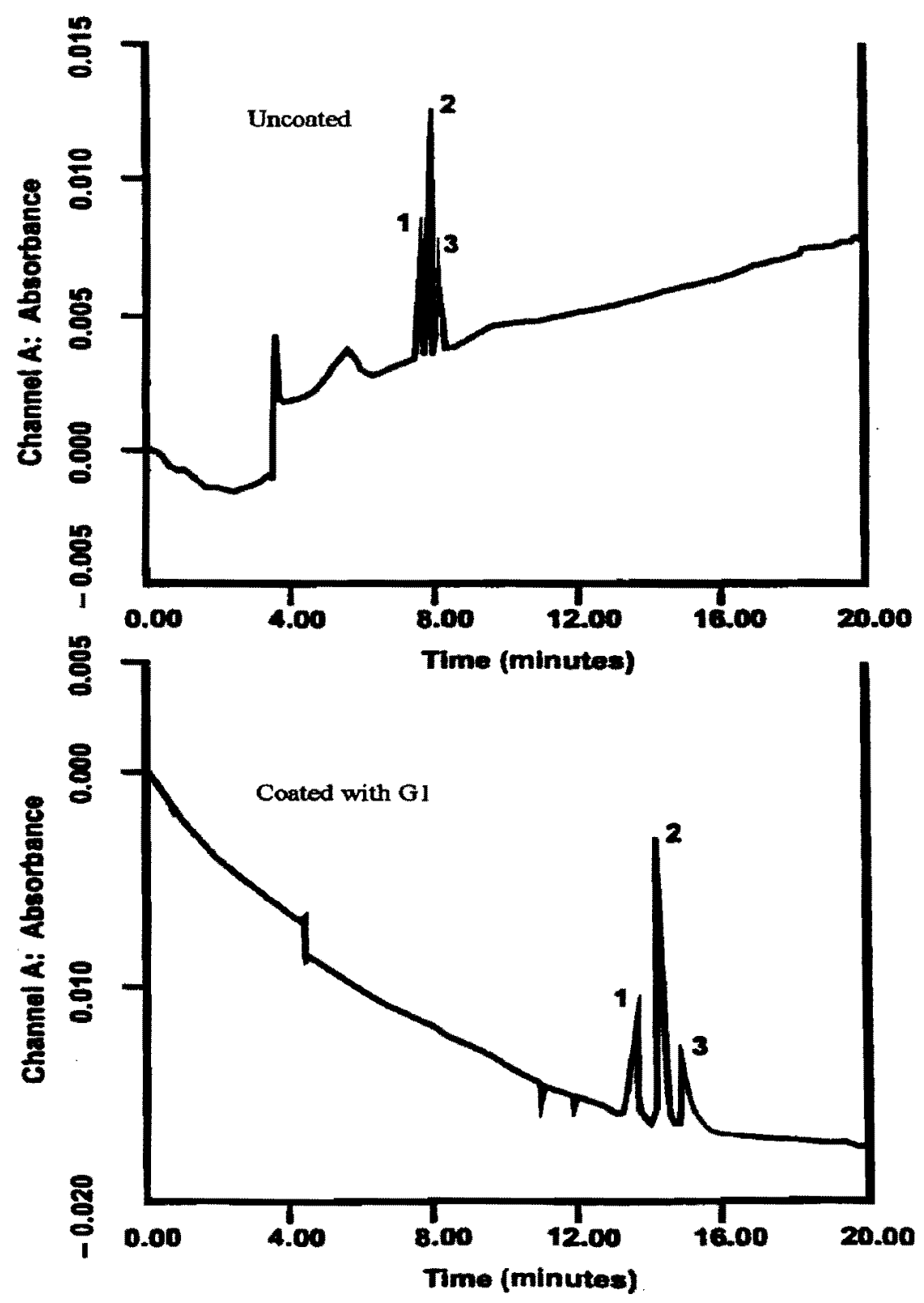

Figure 5-8. Neutral compound electropherograms obtained from uncoated and G1 coated capillaries: (1) naphthalene, (2) phenanthrene and (3) pyrene. 

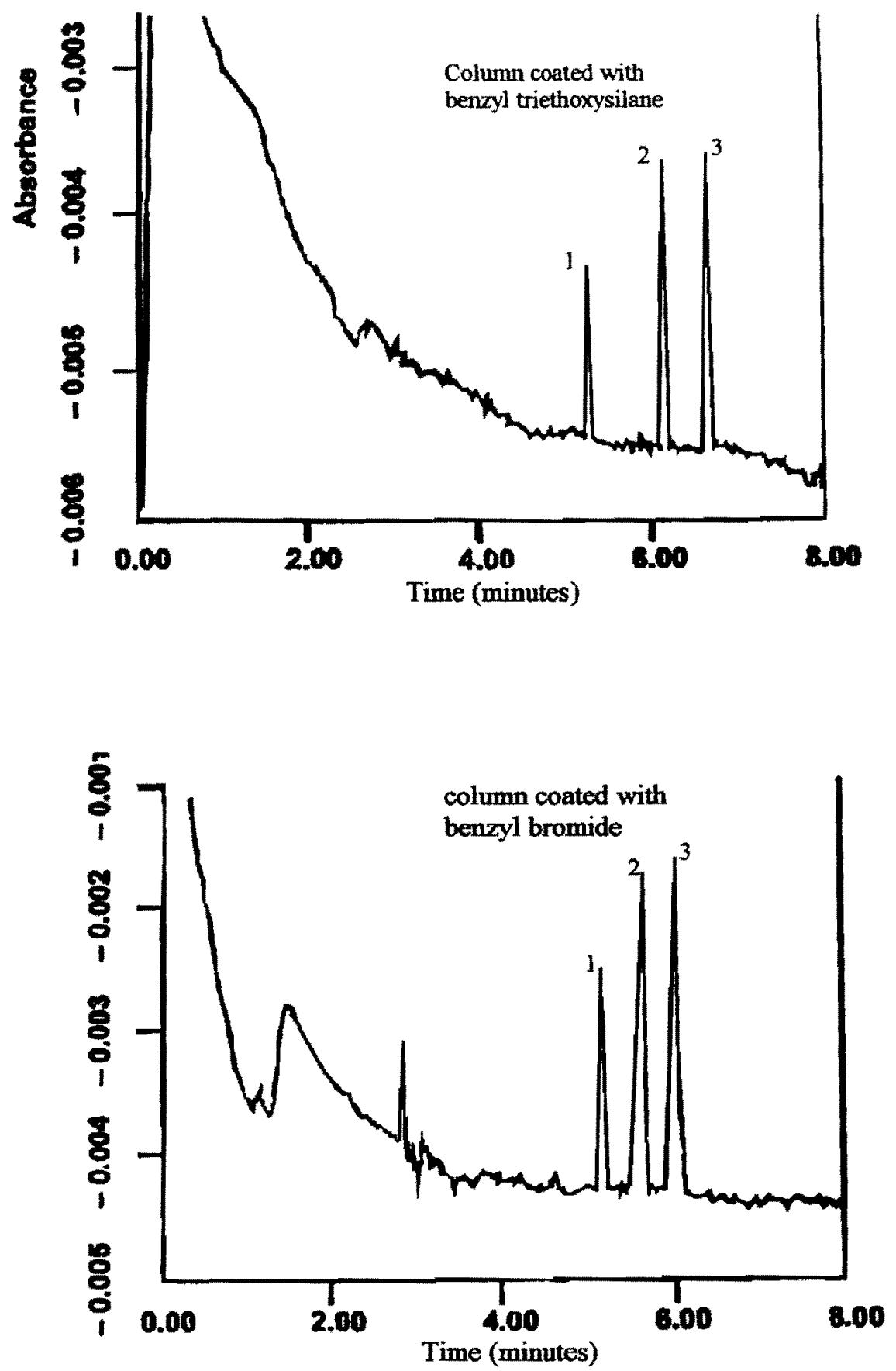

Figure 5-9. Column coating comparison when benzyl triethoxysilane and benzyl bromide are used. (1) benzoic acid, (2) p-hydroxybenzoic acid and (3) phydroxyphenylacetic acid. 
Table 5-2. Resolution comparison between the $\mathrm{Si}-\mathrm{O}-\mathrm{C}$ and $\mathrm{Si}-\mathrm{O}-\mathrm{Si}$ coatings.

\begin{tabular}{|l|l|l|}
\hline Coatings & $\mathrm{R}_{2 \mathrm{t}-1 \mathrm{t}}$ & $\mathrm{R}_{3 \mathrm{t}-2 \mathrm{t}}$ \\
\hline Uncoated & 2.40 & 1.60 \\
Benzyl bromide (Si-O-C) & 1.92 & 1.28 \\
Benzyl triethoxy silane (Si-O-Si) & 6.67 & 3.84 \\
\hline
\end{tabular}

Where, $R_{2 t-1 t}=2\left(t_{2}-t_{1}\right) /\left(W_{2}+W_{1}\right)$.

$R_{2 t-1 t}:$ resolution of peaks 1 and 2.

$R_{3 t-2 t}$ : resolution of peaks 2 and 3 .

\subsubsection{Permanent coating using silane compound as an anchoring agent}

The design and implementation of the synthesis method to anchor the neutral dendrimers to the silanol wall by the formation of $\mathrm{Si}-\mathrm{O}-\mathrm{Si}$ bonds was not a trivial matter. Many silane linkers were considered, but only the two most promising silanes were evaluated: 3-(triethoxysilyl)propyl isocyanate and the 3-amino propyl triethoxysilane. To obtain a specific coating, the desired coating material must be attached to the linker. In the first step, the coupling reaction of the poly(aryl ether) alcohol and 3(triethoxysilyl)propyl isocyanate forms the carbamates which are stable over a $\mathrm{pH}$ range of 4- 8. But to modify the 3-amino propyl triethoxysilane coating, a bromide dendrimer is required. The reaction of this primary amine with a bromide compound is not easily controlled. Tertiary and quaternary amine by-products are usually formed. Columns coated with the amine mixtures produce many undesired effects due to the electrolyte $\mathrm{pH}$. For this reason, the 3-(triethoxysilyl)propyl isocyanate was chosen as the linker. The 
attachment of the poly(aryl ether) alcohol to the isocyanate linker is simple and controllable. In theory, a stoichiometric reaction is predicted. In addition, poly(aryl ether) alcohol is a process intermediate, unlike the poly(aryl ether) bromide which requires a conversion of the corresponding alcohol to the bromide.

Five different carbamates as bonded phases were synthesized (see Chapter 4 , Section 4.4) and studied: the G1 and G2 poly(aryl ether) dendrimers, a C18 alkyl chain, 4-pyridyl carbinol, and benzyl alcohol. The chemical structures of the five carbamates as coating materials are shown in Figure 5-10.

\subsubsection{Preparation of the coated capillary column}

The fused silica capillaries, approximately $400 \mathrm{~cm}$ length, were coiled on a GC capillary column supporter. Using a conventional GC capillary column coating device (Figure 5-11), the capillary was first rinsed with $1 \mathrm{M} \mathrm{KOH}$ for one hour using 25 psi pressure followed by a 20 minute rinse with methanol to prepare the surface of the inner wall. After this, the capillary was flushed with a toluene solution containing the appropriate polymer materials $(2 \% \mathrm{w} / \mathrm{w})$, (either the G1 carbamate, the G2 carbamates, the C18 carbamate, the 4-pyridyl carbinol carbamate or the benzyl carbamate) for 2 hours. The coated capillary was then rinsed with toluene for 20 minutes to remove excess coating material and sealed at both ends with septa. The sealed capillary was placed in a GC oven and heated from $30^{\circ} \mathrm{C}$ to $120^{\circ} \mathrm{C}$ using a temperature program rate of $20^{\circ} \mathrm{C} / \mathrm{min}$. The $\mathrm{GC}$ oven was held at the final temperature for 2 hours. After baking, a gentle flow of Helium (He) at 25 psi pressure was applied to the capillary for 30 minutes at $70^{\circ} \mathrm{C}$ to remove the toluene. The coated capillary column was rinsed with methanol for 
30 minutes and dried in the $\mathrm{GC}$ oven at $70^{\circ} \mathrm{C}$ for 30 minutes with a gentle Helium flow.

The coated capillary is now ready for use or can be stored dry at room temperature.

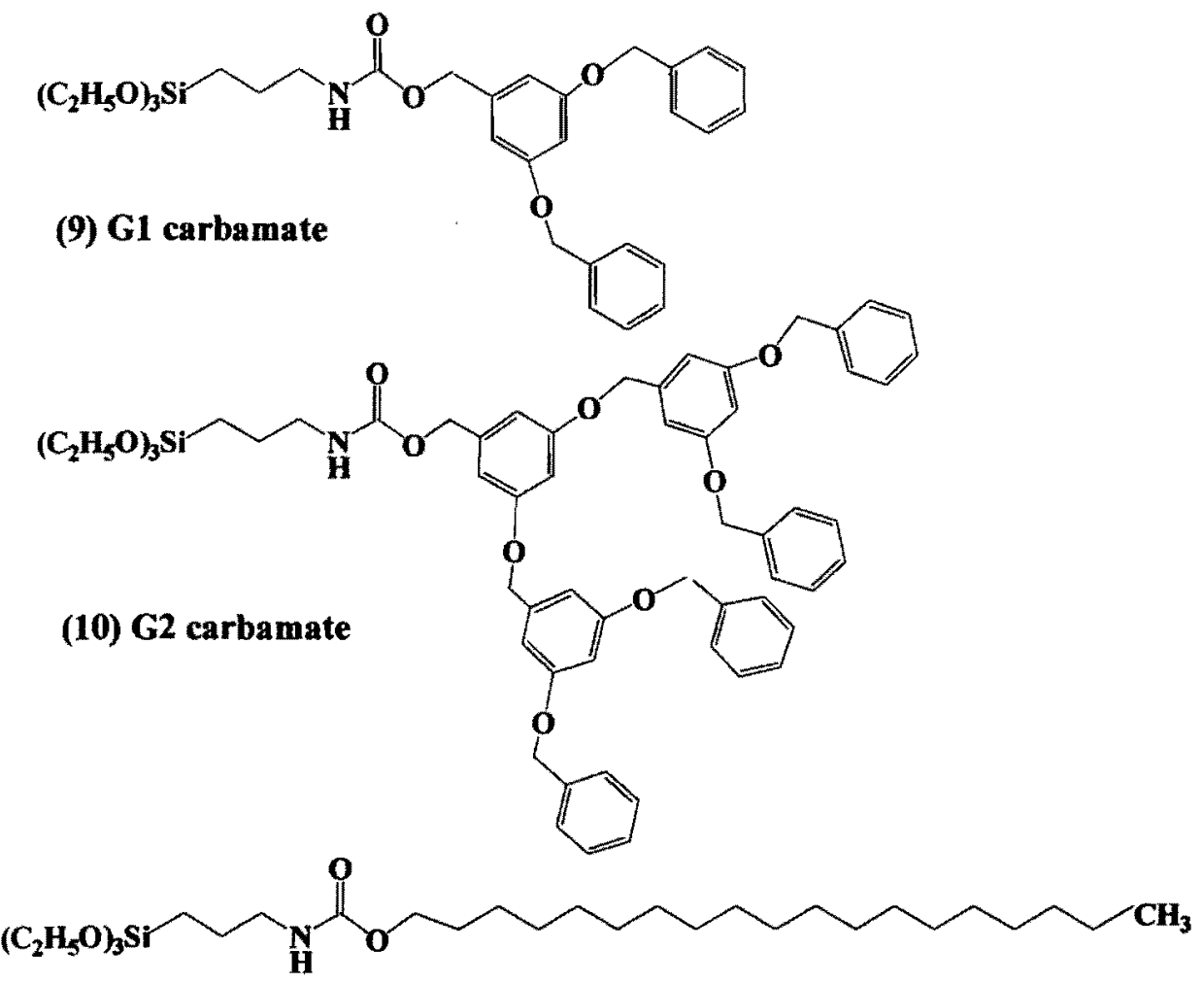

(11) C18 carbamate<smiles>CCOCCCCNC(=O)OCc1ccncc1</smiles>

(12) Pyridyl carbinol carbamate<smiles>CCOCCCCNC(=O)OCc1ccccc1</smiles>

(13) Benzyl carbamate

Figure 5-10. The chemical structure of the five coating materials used for OT-CEC. 


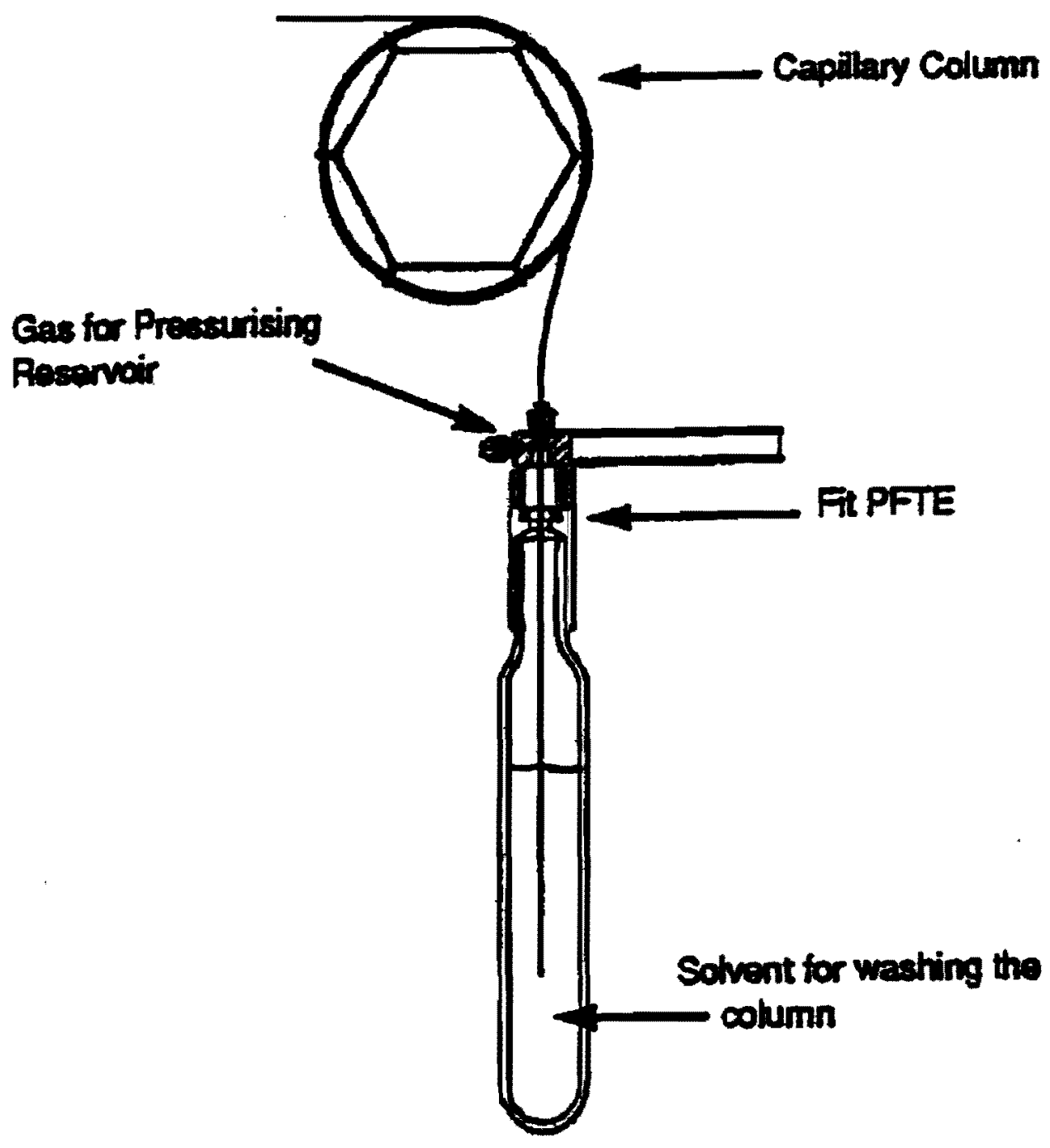

Figure 5-11. Diagram of the capillary coating device. 


\subsection{Surface characterization of the bonded stationary phases.}

It is essential to have a full understanding of the surface coverage for the modified capillary columns to improve the column preparation. However, it is very difficult in practice to directly study the inner surface of coated capillaries with small inner diameters. In this work, we performed studies of the surface modifiers following coating on fused silica discs. Fourier-Transform Spectroscopy (FTIR), Raman spectroscopy and Ultra-Violet spectroscopy methods were employed to assist the optimization of the column coating protocol and to confirm the presence of the functional groups (i.e., dendrimer or pyridyl groups) on the modified surface of the silica disc. All spectroscopic results for the surface characterization, which are presented in this chapter, were obtained from coated fused silica plates prepared by a dip coating method.

\subsubsection{Preparation of dendrimer coatings on fused silica plates. Preparation of the} surface coatings on fused silica plates involved the following steps: (1) pretreatment of the silica plates, (2) the surface coating material is applied by dipping. Fused silica plates were purchased from Edmund Scientific Company, Industrial Optics Division. Each disc is $10 \mathrm{~mm}$ in diameter, $1 \mathrm{~mm}$ thick and polished on both sides. First, the silica discs were treated with $1 \mathrm{M} \mathrm{KOH}$ (submerged in $\mathrm{KOH}$ solution) for about one hour, then were washed with a large amount of purified water from an ultra-pure water purification system (Millipore, Bedford, MA). The clean discs were then dried in an oven at $\sim 180^{\circ} \mathrm{C}$ inside covered Petri dishes. To establish a reproducible surface modification procedure, two representative carbamate coating materials were used in this study: G1 carbamate (6) and the 4-pyridyl carbinol carbamate (9). Solutions of $2 \%, 5 \%$ and $10 \%$ of the 
appropriate carbamates in toluene were prepared. The pretreated silica discs were dipped into the toluene solution, agitated gently, and removed after 2 to 5 minutes. They were rinsed free of excess materials by dipping briefly in toluene. The silane layer was cured for 5 minutes at $110^{\circ} \mathrm{C}$.

\subsubsection{Spectroscopic results. FTIR spectra (Figure 5-12) demonstrate difference} between the non-coated silica plate and the G1 carbamate coated and 4-hydroxymethyl pyridine carbamate coated plates. This was observed in the absorption pattern in the $3800 \mathrm{~cm}^{-1}$ to $3400 \mathrm{~cm}^{-1}$ and $2400 \mathrm{~cm}^{-1}$ to $2000 \mathrm{~cm}^{-1}$. UV spectra (Figure 5-13) obtained for silica plates coated with G1 material had a broad absorption band with $\lambda_{\max }=210 \mathrm{~nm}$, while the 4-hydroxymethyl pyridine carbamate coated plate showed a broad absorption band with $\lambda_{\max }=260 \mathrm{~nm}$. No UV absorption was observed for either a $\mathrm{KOH}$ treated plate or a blank plate. Raman spectra (Figures 5-14, 5-15 and 5-16) of the G1 coated disc indicated a noticeable difference from the 4-pyridyl carbinol carbamate coated disc in the region of $2000 \mathrm{~cm}^{-1}$ to $1800 \mathrm{~cm}^{-1}$.

The results obtained using spectroscopic methods have demonstrated that a thinlayer film was coated onto the fused silica disc using our coating procedure. Another analytical technique, atomic force microscopy (AFM) image was also performed to examine the modified silica disc surface. A silica disc dipped in a $5 \%$ G1 carbamate solution was scanned. However, the surface of this coated plate was found to be too soft to allow the cantilever to sweep across the disc's surface. This observation has provided valuable information for the optimization of the coating procedure. One can postulate the undesired film thickness on the inner surface of a capillary column resulting from the 
treatment with highly concentrated solutions of the coating material. To validate our hypothesis, capillary columns coated with $5 \%$ and $10 \%$ of G1 carbamate solution were evaluated using the standard column preparation procedure. This resulted in column clogging- no flow was observed at the column outlet.

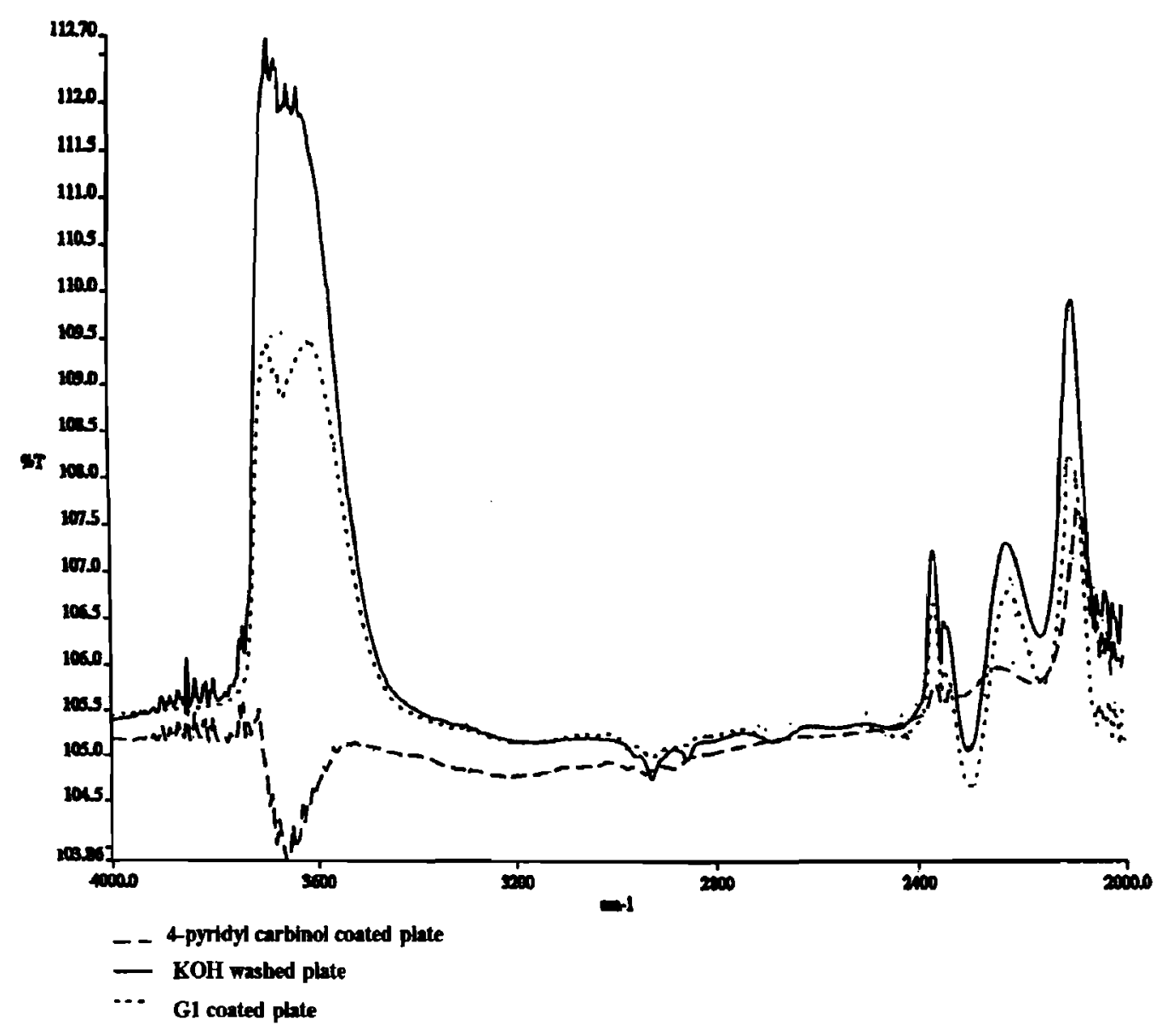

Figure 5-12. FTIR spectra comparison of the G1 and 4-pyridyl carbinol carbamate coated fused silica plates versus an uncoated fused silica plate. 


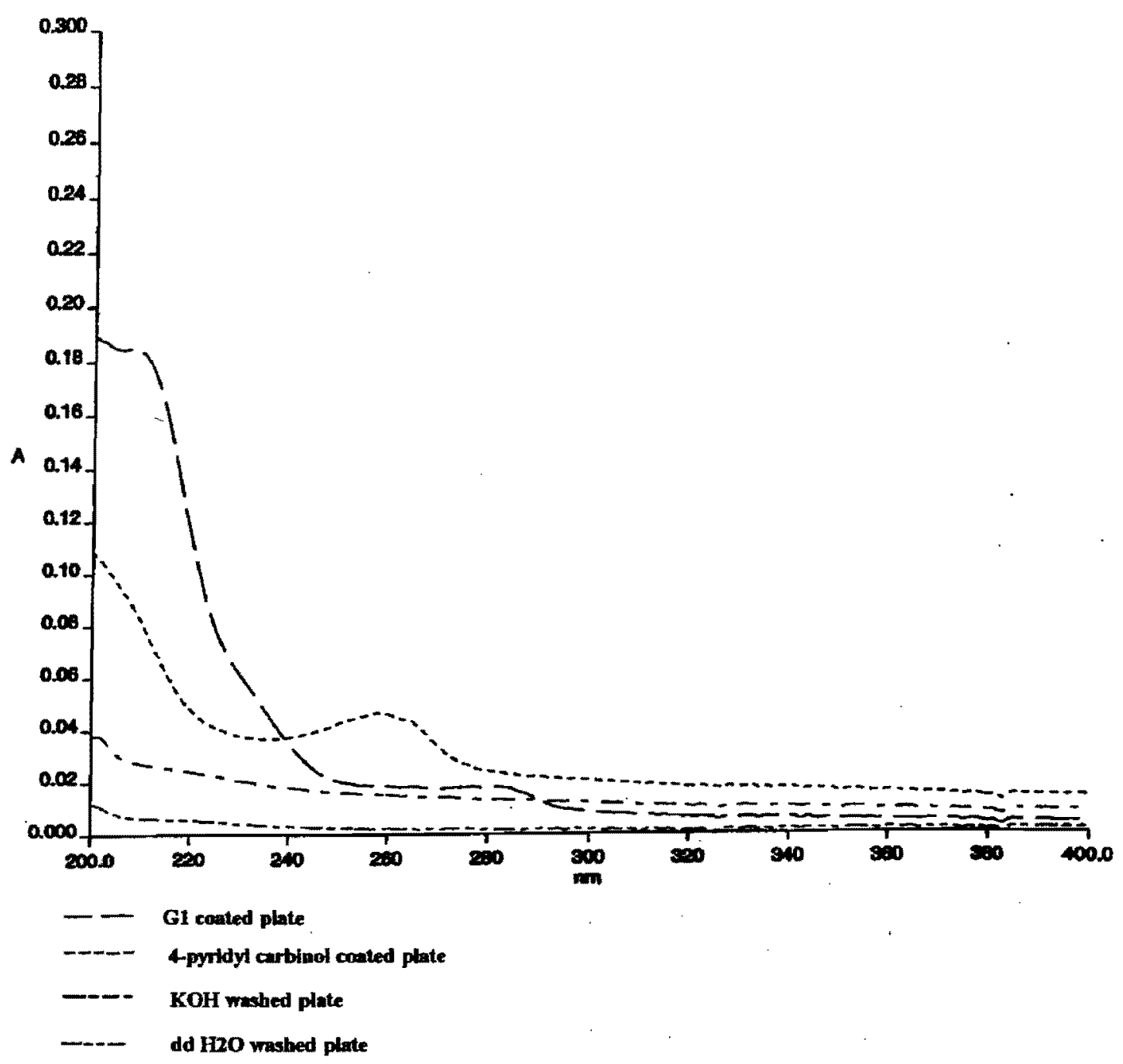

Figure 5-13. UV spectra comparison of the G1 and 4-pyridyl carbinol carbamate coated fused silica discs versus an uncoated silica discs. 


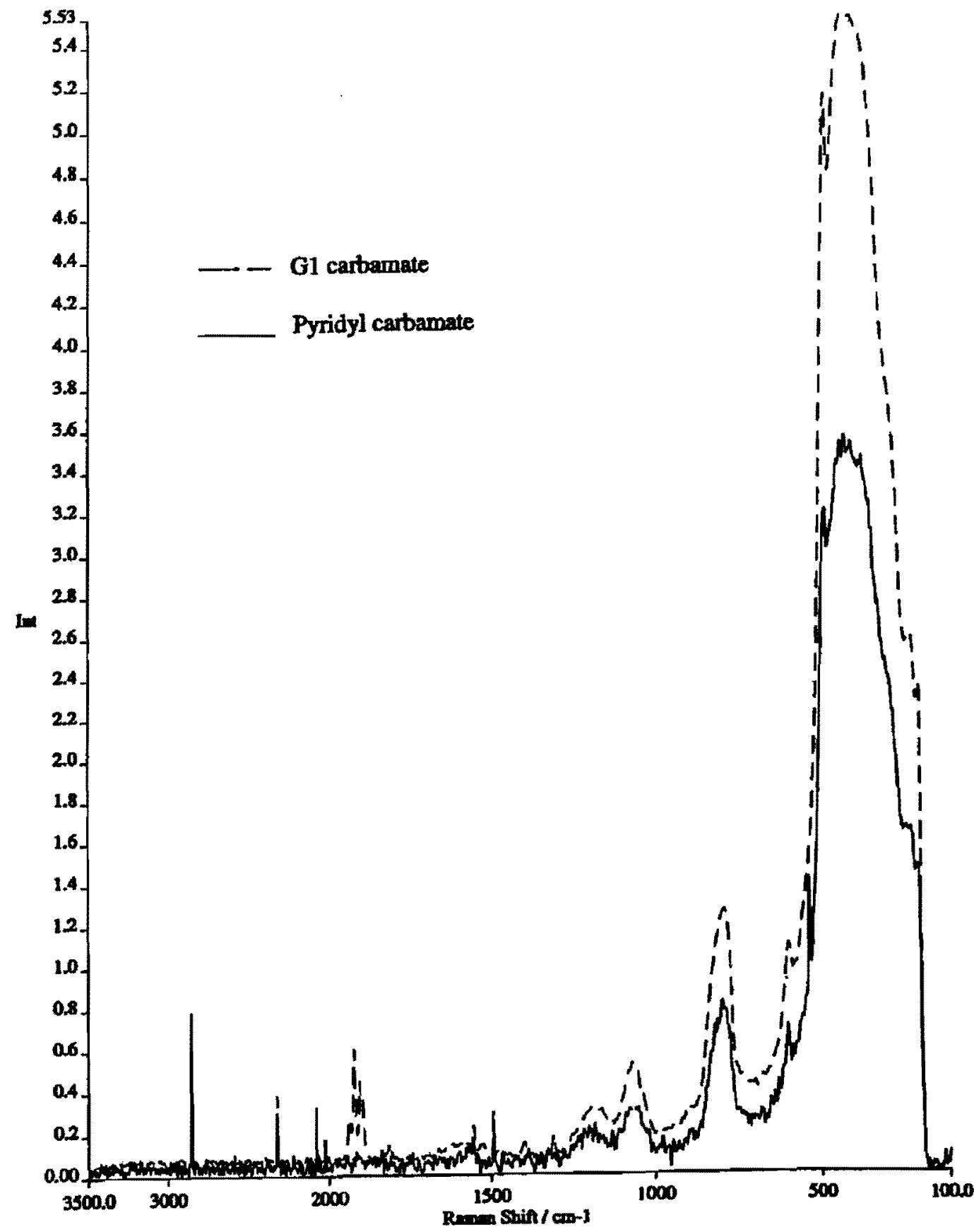

Figure 5-14. Raman spectra comparison of the G1 and 4-pyridyl carbinol carbamate coated fused silica plates. 


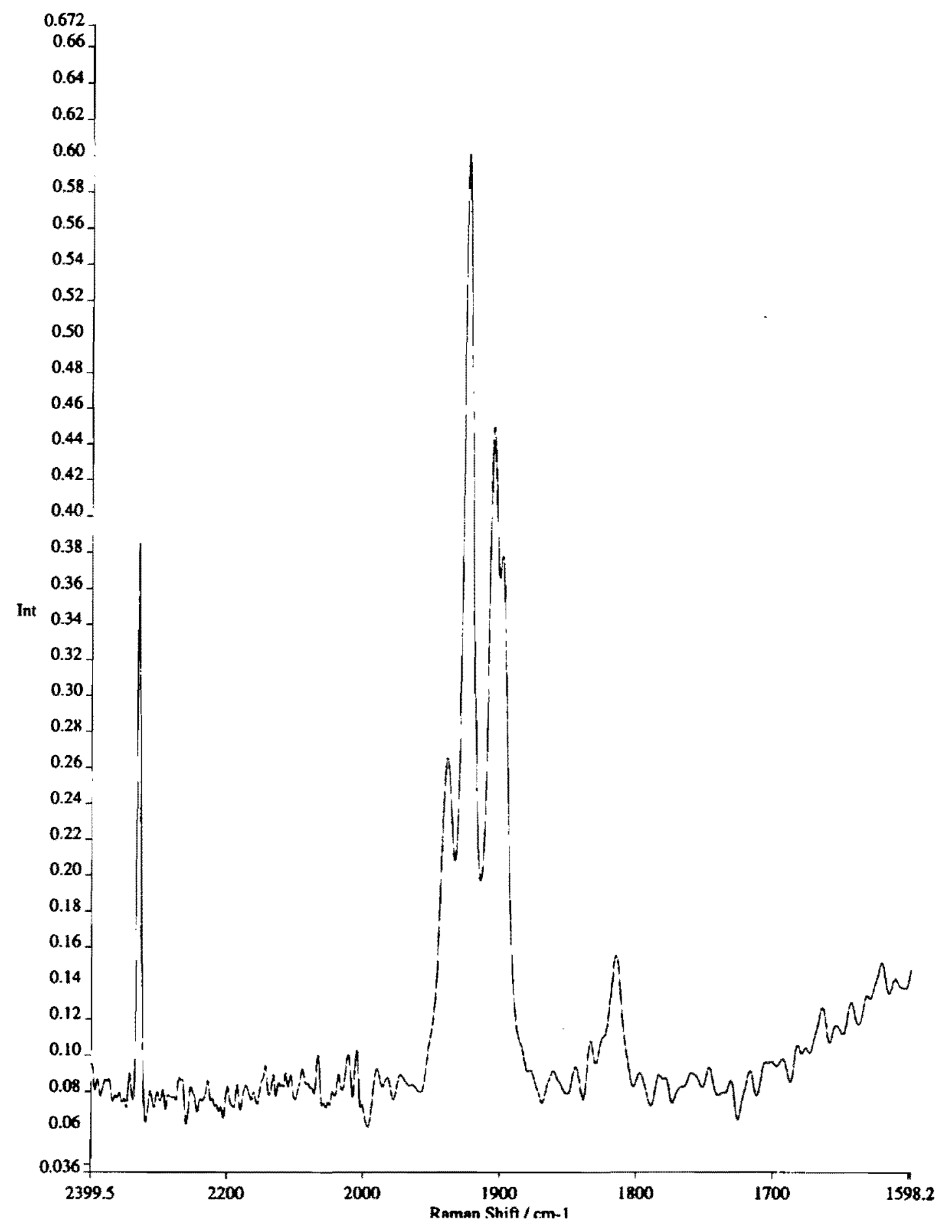

Figure 5-15. Raman spectrum of G1 coated fused silica plate, zoom scan for the wavelength region of $2000 \mathrm{~cm}^{-1}$ to $1800 \mathrm{~cm}^{-1}$. 


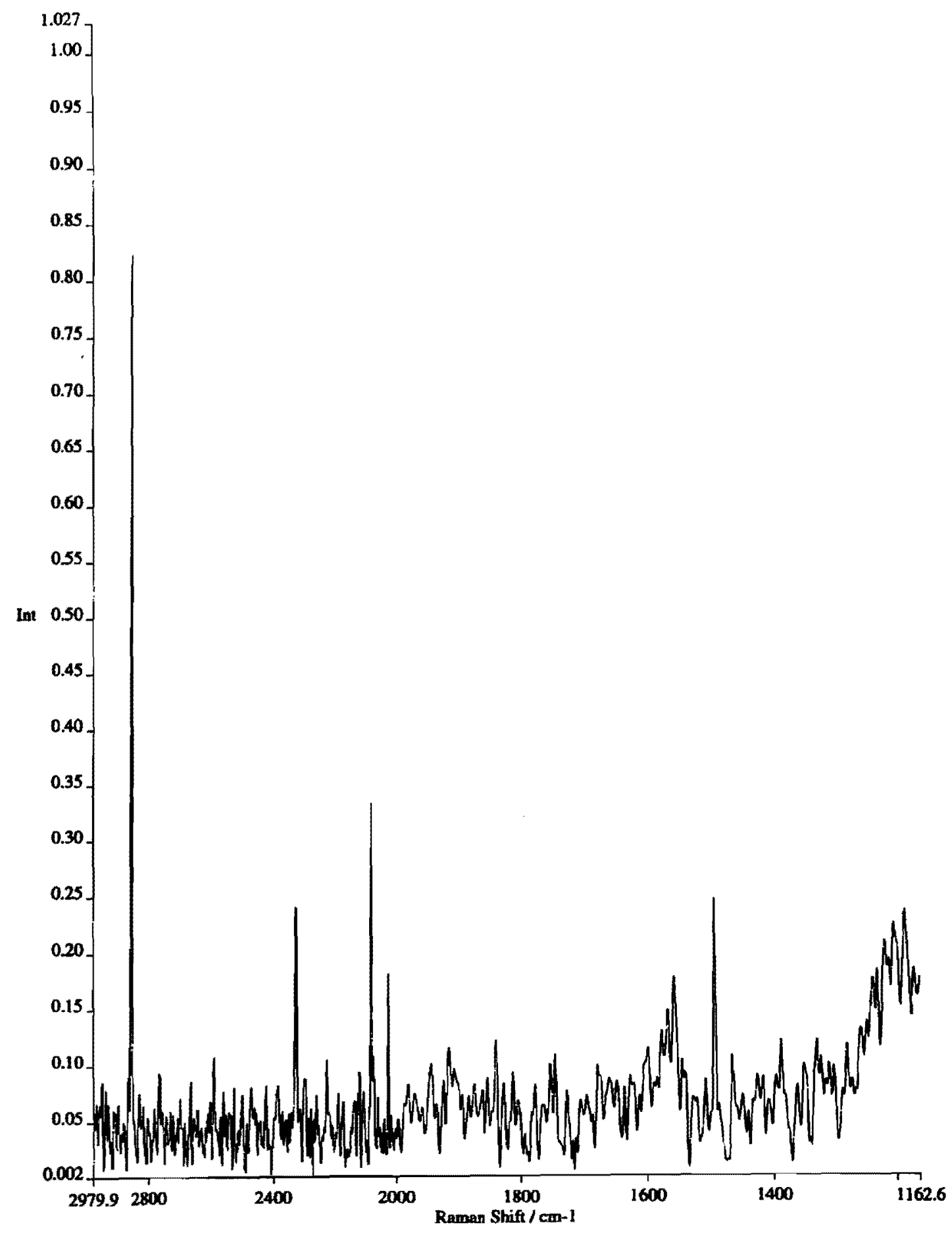

Figure 5-16. Raman spectrum of 4-pyridyl carbinol carbamate coated fused silica plate, zoom scan for the wavelength region of $2000 \mathrm{~cm}^{-1}$ to $1800 \mathrm{~cm}^{-1}$. 


\subsection{Column evaluation.}

5.5.1 Operational conditions. A Beckman P/ACE, model 2050 equipped with on line UV detection and a Hewlett Packard HP ${ }^{3 \mathrm{D}} \mathrm{CE}$ system were used to perform all CEC experiments. A column length of $50 \mathrm{~cm}$, a $20 \mathrm{kV}$ operating voltage and a 5 second pressure injection were used throughout all experiments for consistency.

Prior to first use, the coated column was conditioned according to the following sequence: (1) 30 minutes rinse with methanol, (2) 10 minutes rinse with $\mathrm{ddH}_{2} \mathrm{O}$, and finally (3) 30 minutes rinse with the operating buffer. During each run, CE protocol was followed for the aqueous system: the column was preconditioned with $\mathrm{ddH}_{2} \mathrm{O}(2 \mathrm{~min}$. rinsing), running buffer ( $5 \mathrm{~min}$.), separation time (varied from 10 to 30 minutes), and post-conditioned with $\mathrm{ddH}_{2} \mathrm{O}$ ( $3 \mathrm{~min}$. rinsing). For the organic modified buffer systems, a ddH2O rinse was not needed, and the capillary column was equilibrated with the running buffer for 15 minutes prior to the separation. For each new buffer, the column was then equilibrated with the new buffer system for a 30 minute period.

On-line UV detection was used in all operations. The optical window was prepared by burning off the outer polyimide coating of the capillary after completion of the chemical and thermal treatments involved in column preparation. The effective length to the detector is based on the recommended length for each instrument. Detection was carried out at a wavelength of $214 \mathrm{~nm}$.

5.5.2 Buffer solutions. Several buffer solutions were used to operate capillaries over the $\mathrm{pH}$ range $4-9.3$ : phosphate buffer at $\mathrm{pH} 4.03$ and 7.0, MES (2-[N-morpholino] 
ethanesulfonic acid) buffer at $\mathrm{pH}$ 6.0, TRIS (tris(hydroxymethyl-aminomethane)) buffer at $\mathrm{pH} 8.3$, and borate buffer at $\mathrm{pH} 9.3$.

\subsection{Results}

5.6.1 EOF. The suppression of EOF was observed for the G1 and C18 coated columns using thiourea as a neutral marker. A lower EOF with the G1 coating versus the $\mathrm{C} 18$ coating was obtained over the entire $\mathrm{pH}$ range 4 -9.3. Near zero EOF was obtained when capillary columns coated with the G2 carbamate were used. Table 5-3 contains the calculated EOF over the $\mathrm{pH}$ range of $4.0-9.3$ for the coated and uncoated capillary columns.

Capillary columns coated with 4-pyridyl carbinol carbamate have a reversed EOF direction when operated at $\mathrm{pH}$ 4.0. The electroosmotic flow observed is $1.10 \times 10^{-4} \mathrm{~cm}^{2} \mathrm{v}^{-1} \mathrm{~s}^{-1}$ which is $30 \%$ less when it is compared to the $\mathrm{G} 1$ coated column and $65 \%$ less when it is compared to the $\mathrm{C} 18$ coated column using the same conditions. When the polarity was reversed, an EOF of $2.92 \times 10^{-4} \mathrm{~cm}^{2} \mathrm{v}^{-1} \mathrm{~s}^{-1}$ was observed at $\mathrm{pH}$ 4.0.

5.6.2 Evaluation of coated capillaries with neutral compounds. Neutral species can not be separated by traditional CE methodology due to the lack of electrophoretic mobility. The separation of toluene, naphthalene and phenanthrene demonstrates the resolution and selectivity obtained with the $\mathrm{Gl}$ coated capillary column prepared using the method we developed in this research. Figure 5-17 shows the separation of toluene from the other polyaromatic compounds. The separation was achieved using a $54 \mathrm{~cm}$ 
length, $50 \mu \mathrm{m}$ capillary, at $15 \mathrm{kV}$, in a $\mathrm{pH} 6.0,5 \mathrm{mM}$ MES buffer modified with $30 \%$ methanol as the eluent. The column temperature was maintained at $15^{\circ} \mathrm{C}$. The effect of different coating materials on the migration time of the analytes was studied using 2'-fluoroacetanilide, a neutral analyte. It was observed that under the same CEC running conditions, the migration time of 2'-fluoroacetanilide was 1.65 times longer for a G1 coated column than a $\mathrm{C} 18$ coated column. The migration time of 2 '-fluoroacetanilide on a 4-pyridyl carbinol coated column was 1.25 times longer compared with G1 coated and 2.0 times longer compared with C18 coated columns. Table 5-4 showed the migration time of 2'-fluoroacetanilide observed at two different $\mathrm{pH}$ conditions.

\subsubsection{Evaluation of coated capillaries with basic amine substances. In traditional}

$\mathrm{CE}$, the strong EOF for positively charged species, such as basic amines, can cause too rapid migration, resulting in either no retention or loss of resolution. Another problem associated with the separations of basic amine compounds is the strong electrostatic interaction of the amines with the negatively charged siloxane wall which causes peak distortion.

A mixture of two basic amine compounds, phenyl ethylamine, 2, 6-difluoroaniline and a neutral compound, 2'-fluoroacetanilide, was examined to evaluate the performance of three different coated capillary columns; G1, C18 and 4-pyridyl carbinol coated columns. The CEC separation was performed using a $54 \mathrm{~cm}$ length, $50 \mu \mathrm{m}$ capillary, at $15 \mathrm{kV}$, in a pH 4.0, $10 \mathrm{mM}$ phosphate buffer without organic modifier. The pKa of phenyl ethylamine is 9.84 , and at $\mathrm{pH} 4.0$ it is protonated and migrates as a cation. This positively charged amine is separated from the 2, 6-difluoroaniline and 
2'-fluoroacetanilide. At this writing, there are no $\mathrm{pKa}$ references giving for 2,6difluoroaniline. However we have found the pKa values of analogs of aniline compounds in CRC Handbook of Chemistry and Physics ${ }^{30}$ : aniline, $\mathrm{pKa}=4.63$; 2-fluoroaniline, $\mathrm{pKa}=3.20 ; 2,4$-dichloroaniline, $\mathrm{pKa}=2.05$ and 3,5-dibromoaniline, $\mathrm{pKa}=2.34$. Based on these information, we predicted that the pKa of 2, 6-difluoroaniline is between 2 to 3 . Therefore, 2, 6-difluoroaniline remains as a neutral molecule in a $\mathrm{pH} 4.0$ buffer. Under this condition, the separation of 2, 6-difluoroaniline and 2'-fluoroacetanilide is not achieved with the G1 or the C18 coated columns. Figure 5-18 shows the separation of the phenyl ethylamine from the 2, 6-difluoroaniline and 2'-fluoroacetanilide on G1 and C18 coated columns.

5.6.4 Evaluation of coated capillaries with basic proteins. Adsorption of positively charged proteins onto the walls of fused-silica capillaries is a problem in CE. Extensive research has been made to rectify this problem. In our study, a protein test mixture consisting of lysozyme, cytochrome $\mathrm{C}$ and ribonuclease $\mathrm{A}$ was separated using a $\mathrm{pH} 6.0$ MES buffer, $\mathrm{pH} 7.0$ phosphate buffer, and a pH 8.3 TRIS buffer. The best resolution of the three proteins was found at $\mathrm{pH}$ 6.0. The electropherograms of the protein separation on a G1 column at different pHs are shown in Figure 5-19.

5.6.5 Evaluation of coated capillaries with anions. Capillary electrophoresis is most applicable for both inorganic and organic anions. The retention of the negative charged species is based on the net electrophoretic mobility which is the difference between the bulk EOF and the anion's electrophoretic mobility. However, in CEC, the separation of 
the charged species is effected by both electrophoretic mobility and partitioning. Typically, anions are separated at higher $\mathrm{pH}$. In this study, some adverse effects were observed when an anion test mixture was examined at $\mathrm{pH}=9.30$. The retention of phenylacetic acid was strongly affected with both G1 and 4-pyridyl carbinol coated columns. Figure 5-20 shows the electropherograms of an anion test mixture using different column coatings. The bottom electropherogram was obtained using reversed polarity.

5.6.6 Evaluation of the selectivity effect on structural isomers. The separation of regio-isomers is always a challenge for HPLC separations. Since the CEC separation mechanism is based on both the partitioning of the analytes with the stationary phases and the electrophoretic mobility of the analytes, the selectivity of the coatings synthesized for this work were evaluated for the separation of two structural isomers. The compounds studied were isomers of epinephrine and pyrrolopyridine.

Unexpected results were obtained for epinephrine. Two peaks were observed for this amine when the electropherograms were obtained with the G1 and 4-pyridyl carbinol coated capillary columns, Figure 5-21. In contrast, only one peak was obtained with the uncoated and $\mathrm{C} 18$ coated capillary columns. Using the G1 coated capillary column, the epinephrine separation was investigated using buffers at $\mathrm{pH} 4,7,8.3$, and 9.3, Figure 522. The optimum $\mathrm{pH}$ of 4.0 was found for the epinephrine separation when the G1 coated capillary was used. The observation of 2 epinephrine peaks is unusual since this optically active compound (i.e. chiral) should give a single response in an achiral environment. An explanation will be provided in Chapter 6 Discussion. Both regio- 
isomers of pyrrolopyridine, the $(3,4 b)$ and $(2,3 c)$, were separated with an uncoated, C18 coated, and $\mathrm{G} 1$ coated capillary columns at $15 \mathrm{kV}$ in a pH $4.0,10 \mathrm{mM}$ phosphate buffer. The G1 coated column provided the best separation, Figure 5-23.

Table 5-3. The experimental EOF values at different $\mathrm{pH}$ using thiourea as a neutral marker.

\begin{tabular}{|lllc|}
\hline & & \multicolumn{2}{c|}{ EOF $\left(\mathrm{cm}^{2} \mathrm{v}^{-1} \mathrm{~s}^{-1} \times 10^{-4}\right)$} \\
\hline PH & G1 & C18 & uncoated fused silica column \\
\hline 4.03 & 1.62 & 3.20 & 3.13 \\
7.00 & 2.69 & 3.27 & 4.12 \\
8.30 & 3.23 & 4.92 & 6.51 \\
9.30 & 3.30 & 3.58 & 9.40 \\
\hline
\end{tabular}

Table 5-4. The migration time of 2'-fluoroacetanilide

\begin{tabular}{|c|c|c|c|}
\hline & \multicolumn{3}{|c|}{ Migration time in minutes } \\
\hline & $\mathrm{G} 1$ & $\mathrm{C} 18$ & Pyridyl carbinol \\
\hline \hline $\mathrm{pH}=4.03$ & 11.50 & 6.35 & 13.20 \\
$\mathrm{pH}=7.00$ & 7.73 & 6.50 & 11.00 \\
\hline
\end{tabular}


<smiles>c1ccccc1</smiles>

Toluene<smiles>c1ccc2ccccc2c1</smiles>

Naphthalene

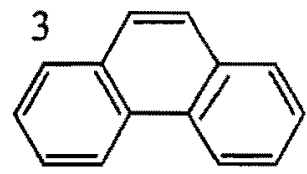

Phenanthrene

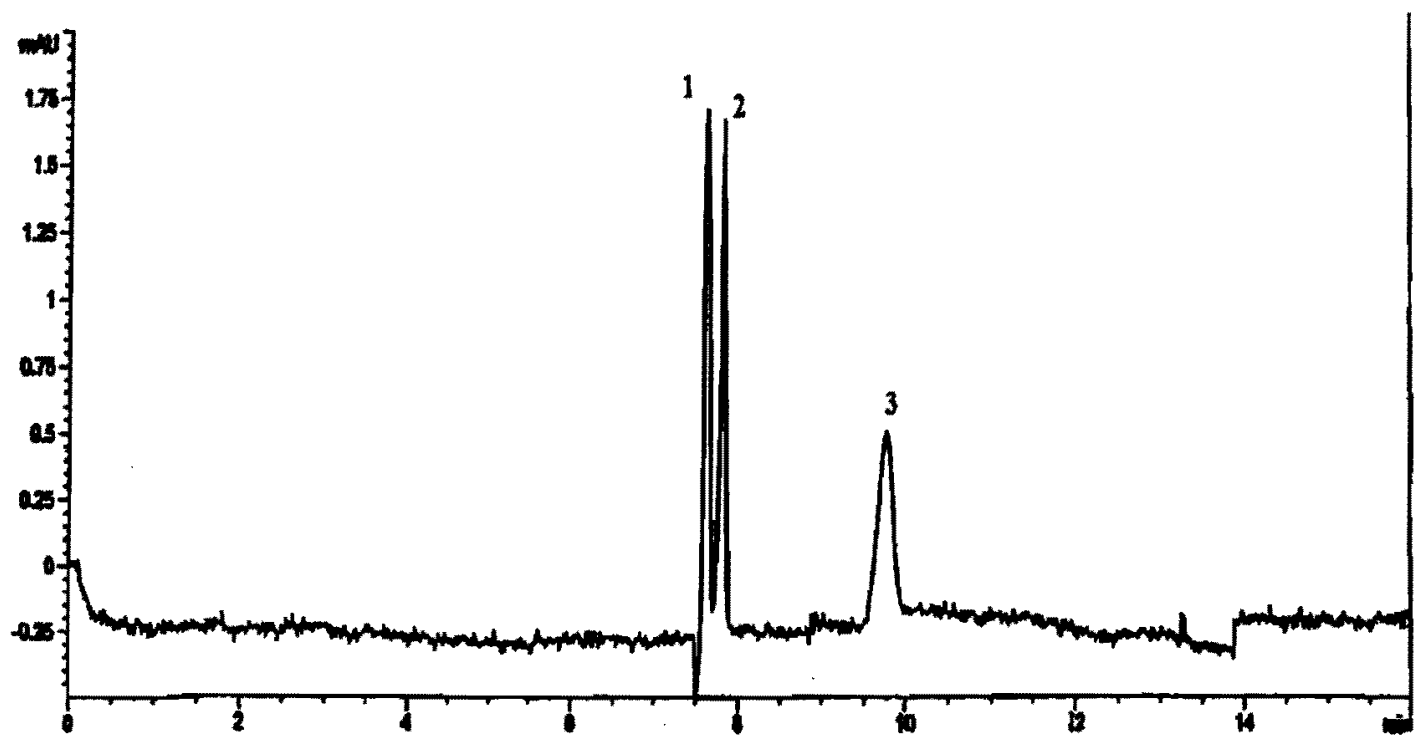

Figure 5-17. The separation of toluene, naphthalene and phenanthrene, obtained with G1 coated capillary column. 
<smiles>CC(N)c1ccccc1</smiles>

Phenyl ethylamine
2<smiles>Nc1c(F)cccc1F</smiles>

2, 6-Difluoroaniline 2'-Fhoroacetanilide<smiles>CC(=O)Nc1ccccc1F</smiles>


Figure 5-18. Electropherograms for the mixture of the phenyl ethylamine, 2, 6difluoroaniline and 2'-fluoroacetanilide. 


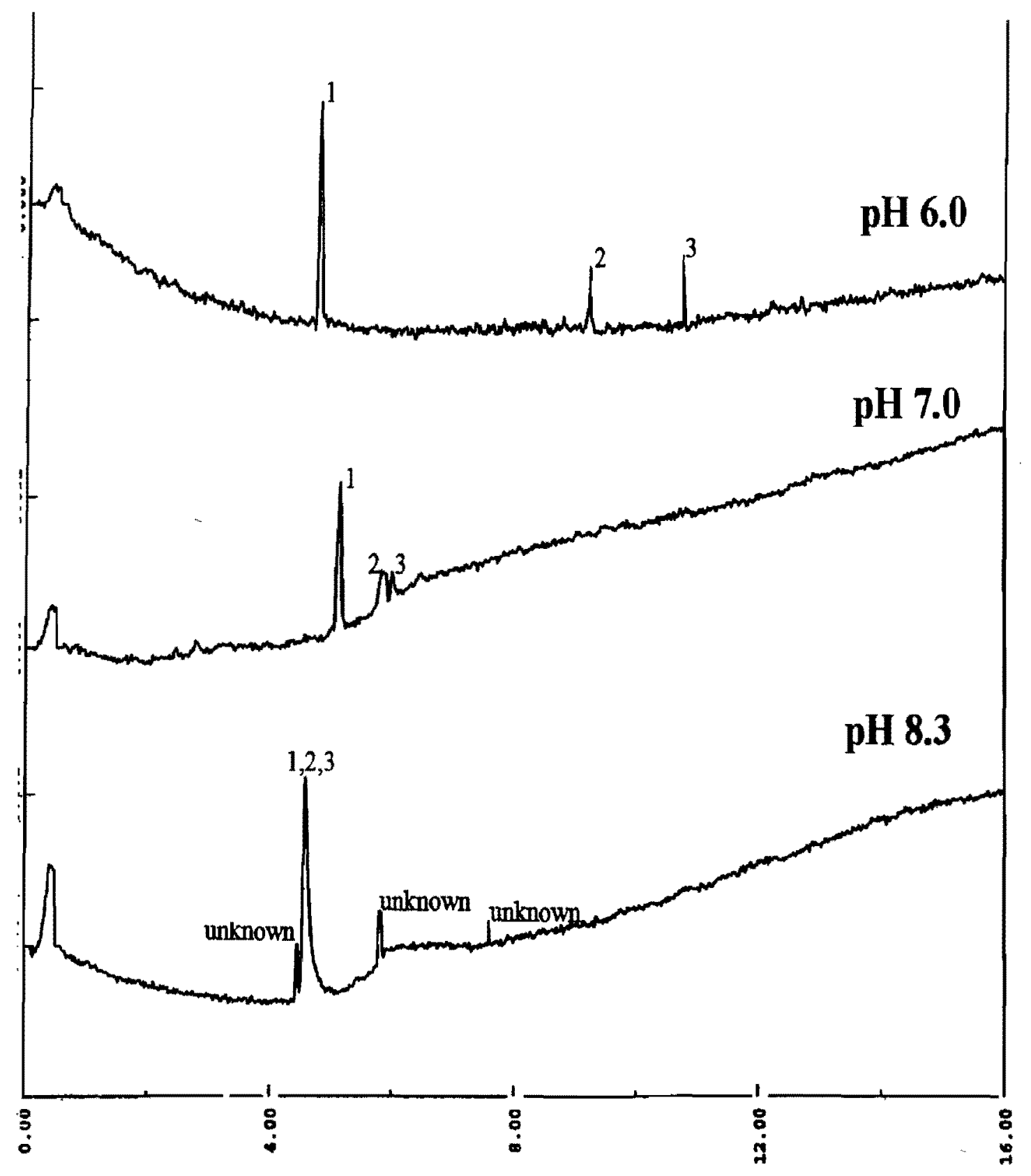

Figure 5-19. Electropherograms of the protein mixture: (1) lysozyme, (2) cytochrome C, and (3) ribonuclease A, using a Gl coated capillary column for separation at $\mathrm{pH} 6.0,7.0$ and 8.3 . 
<smiles>O=C(O)c1ccccc1</smiles>

Benzoic acid<smiles>O=C(O)c1ccc(O)cc1</smiles>

p-Hydroxybenzoic acid<smiles>O=C(O)Cc1ccc(O)cc1</smiles>

p-Hydroxyphenyl acetic acid
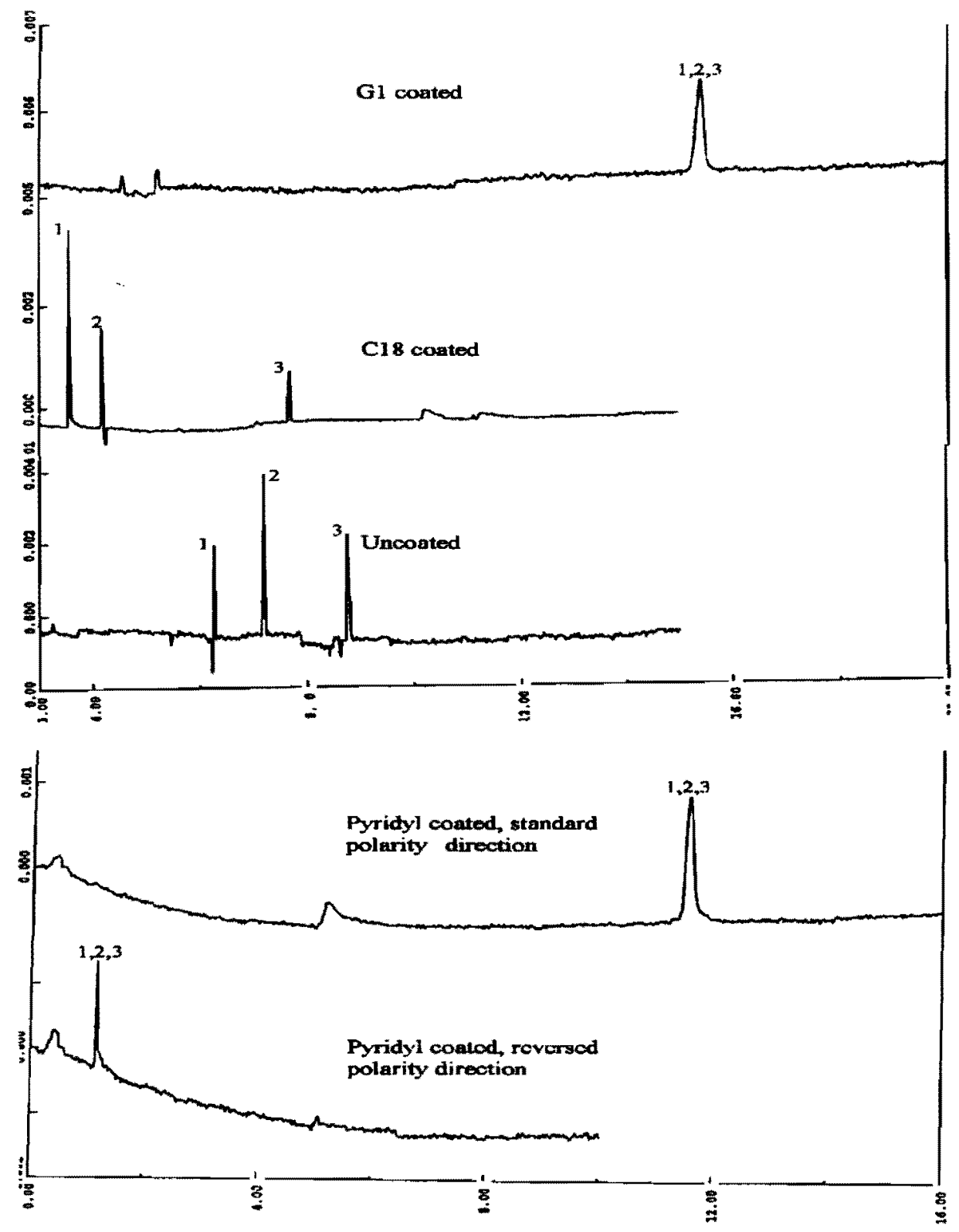

Figure 5-20. Anion test mixture electropherograms migration order: benzoic acid, phydroxybenzoic acid and p-hydroxyphenylacetic acid. 

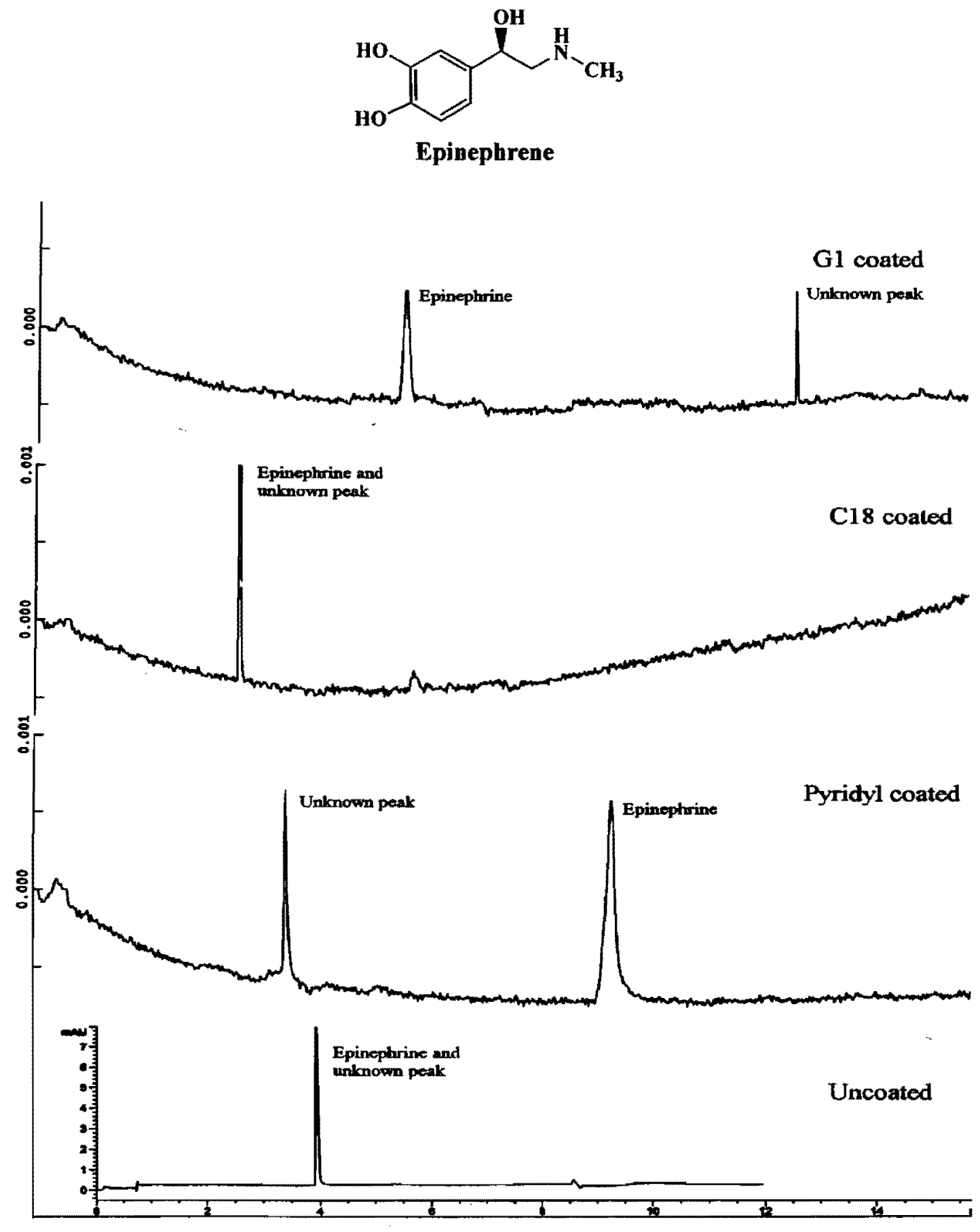

Figure 5-21. Epinephrine electropherograms obtained using uncoated, 4-pyridyl carbinol, C18 and G1 coated capillary columns. 


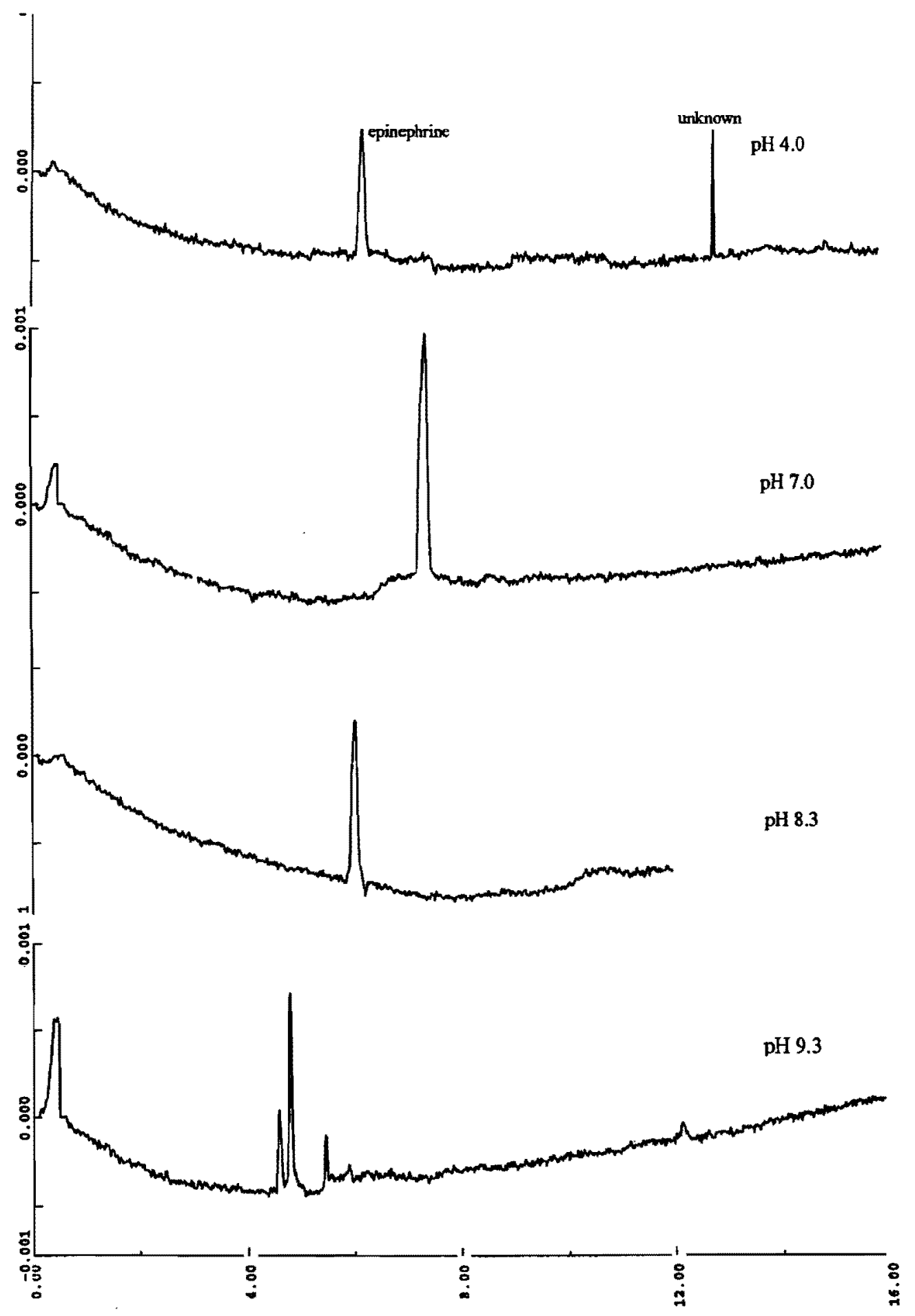

Figure 5-22. Epinephrine electropherogram obtained at different pHs using a G1 coated column. 
$\overbrace{N-2 H C l}$

(3, 4 b)Pyrrolopyridine

Hydrochloride

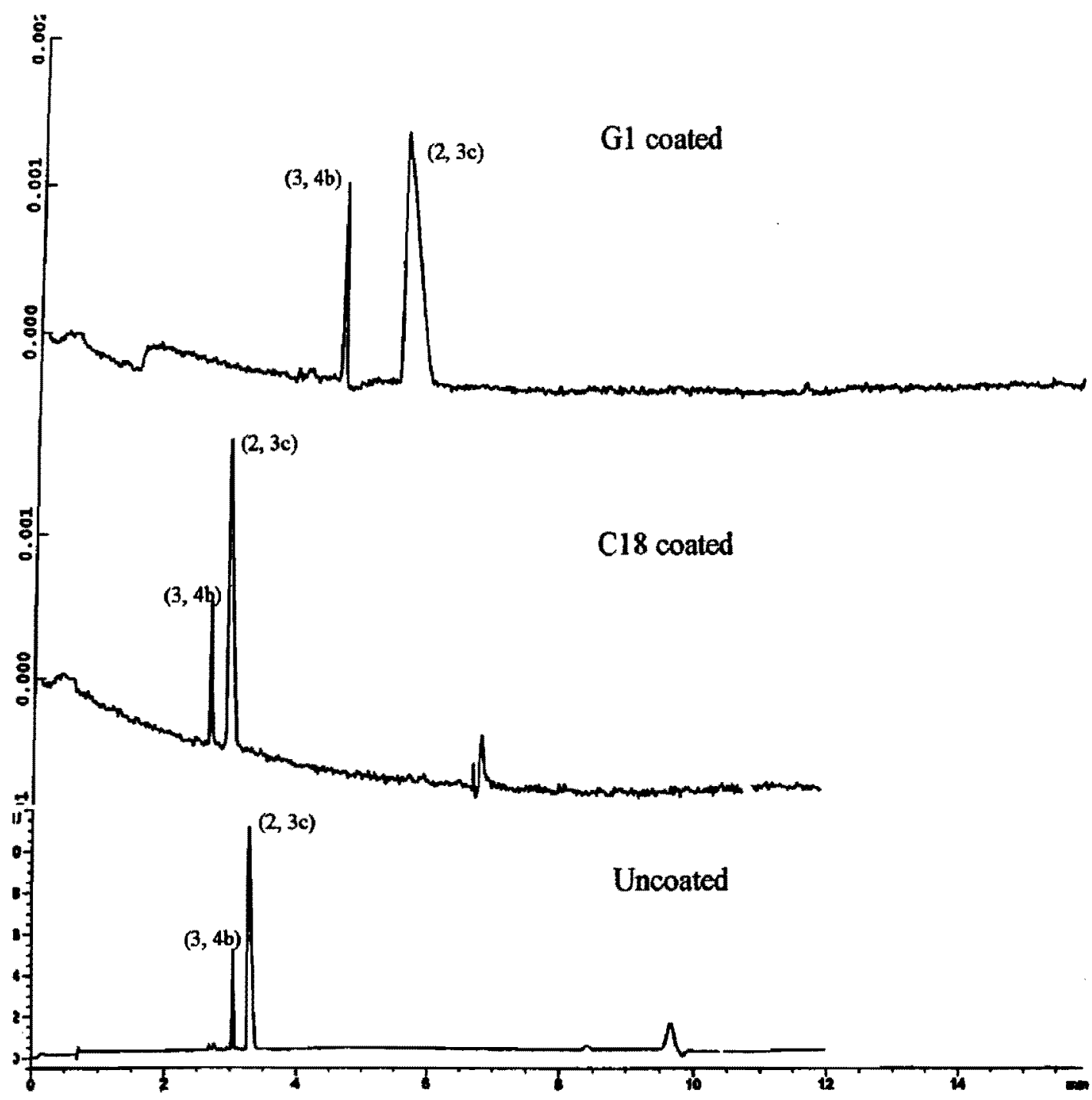

Figure 5-23. Pyrrolopyridine electropherograms obtained with G1, C18 and uncoated capillary columns at $15 \mathrm{kV}$ in a $\mathrm{pH} 4.0$ phosphate buffer. 


\subsection{References for Chapter 5}

1) Dittmann, M. M.; Wienand, K.; Bek, F.; Rozing, G. P. LC-GC 1995, 13, 800.

2) Yan, C.; Daddo, R.; Zhao, H.; Zare, R. N.; Rakestraw, D. J. Anal. Chem. 1995, 67.

3) Smith, N. W.; Evans, M. B. Chromatographia 1994, 38, 649.

4) Gordon, D. B.; Lord, G. A.; Jones, D. S. Rapid Commun. Mass Spectr. 1994, 8, 544.

5) Schmeer, K.; Behnke, B.; Bayer, E. Anal. Chem. 1995, 67, 3656.

6) Bruin, G. J. M.; Tock, P. P. H.; Kraak, J. C.; Poppe, H. J. Chromatogr. 1990, 517, 557.

7) Guo, Y.; Colón, L. A. Anal. Chem. 1995, 67, 2511.

8) Guo, Y.; Colón, L. A. J. Microcol. Sep. 1995, 7, 485.

9) Newkome, G. R.; Moorefield, C. N.; Vogtle, F. 1996.

10) Colón, L. A.; Guo, Y.; Fermier, A. Anal. Chem. News and Features, August 1, $1997,461 \mathrm{~A}$.

11) Pesek, J. J.; Matyska, M. T.; Mauskar, L. J. Chromatogr. A 1997, 763, 307.

12) Marina, M. L.; Crego, A. L.; Gonzalez, A. Critical Reviews in Analytical Chemistry 1996, 26, 261.

13) Scott, R. P. W. "Liquide Chromatography for the Analyst"; Marcel Dekker: New York 1994.

14) Jorgenson, J. W.; Lukacs, K. D. Science 1983, 222, 266-272.

15) Dluzneski, P. R.; Jorgeson, J. W. J. High Resolut. Chromatogr. Commun. 1988, $11,332$. 
16) Poppe, H.; Kraak, J. C.; van Berkel, O. J. Chromatogr. 1990, 449, 345.

17) Gohlin, K.; Larsson, M. J. Chromatogr. 1993, 645, 41.

18) Ruan, Y.; Kraak, J. C.; Poppe, H. Chromatographia , 35, 597.

19) Poppe, H.; Kraak, J. C.; Swart, R. J. Chromatogr. 1994, 670, 25.

20) Tsuda, T.; Hibi, K.; Takeuchi, T.; Nakanishi, T.; Ishii, D. J. Chromatogor. 1979, $175,105$.

21) Tsuda, T.; Ishii, D.; Takeuchi, T. J. Chromatogr. 1979, $185,73$.

22) Tsuda, T.; Tsuboi, K.; Nakagawa, G. J. Chromatogr. 1981, 214, 283.

23) Yeung, E. S.; Pfeffer, W. D. Anal. Chem. 1990, 62, 2178.

24) Schurig, V.; Mayer, S. J. of Liq. Chroma. 1993, 16, 915-931.

25) Tock, P. P. H.; Stegeman., G. R.; Peerboom, R.; Poppe, H.; Kraak, J. C.; Unger, K. K., Chromatographia 1987, 24, 617.

26) Crego, A. L.; Diez-Masa, J. C.; Dabrio, M. V. Anal. Chem. 1993, 65, 1615.

27) Brinker, C.; Scherer, G. W. Sol-Gel Science, Academic: Boston, 1990.

28) Guo, Y. C., Colón, L. A. Electrophoresis 1995, 16, 493.

29) Guo, Y. C., Colón, L. A., J. Microcol. Sep. 1995, 7, 485.

30) Lide, D. R., "CRC Handbook of Chemistry and Physics”, 1995, CRC Press, Inc. 


\section{CHAPTER 6}

\section{Discussion and Conclusion}

\subsection{Dendrimer coated columns}

Our objective was to explore the potential of using dendritic polymers as a bonded stationary phase in OT-CEC and to develop a simple but reproducible method for the preparation of surface modified capillary columns with controlled electroosmotic flow. By convention, the stationary phases commonly used in OT-CEC are prepared by chemically bonding the functional groups onto the inner walls of fused silica capillaries through siloxane linkages. To increase the surface area of the inner walls and phase ratio, two approaches may be used. The silica surface may be etched or a porous silica layer is

deposited on the inner walls. ${ }^{1-4}$ The roughened silica surfaces were then chemically bonded with desired dendrimers via $\mathrm{Si}-\mathrm{O}-\mathrm{Si}$ bonding using benzyl triethoxysilane as an anchoring agent.

The effect of different sizes, G0 and G1 dendrimers, on the peak resolution and the retention behavior of the analytes were investigated by using the standard procedure for $\mathrm{CE}$ analysis. The resulting electropherograms showed that the electroosmotic flow was suppressed when the G0 and G1 poly(aryl ether) dendrimer coated columns were compared to an uncoated column and for some applications the resolution improved using a dendrimer coated column (see Chapter 5, Figure 5-6). The coated column's electropherograms showed an increased migration time which could indicate two possible effects: (1) suppression of the electroosmotic flow and (2) the analyte partitioning effect. The apparent mobilities, $\mu_{\mathrm{app}}$, were calculated from the migration times observed in the experiment. The $\mu_{\text {app }}$ data are presented in Chapter 5, Table 5-1. The migration time 
increases with increasing size of the dendrimer was observed. A reduced EOF was found, when the G0 and G1 poly(aryl ether) dendrimer coated columns were compared to an uncoated column.

To further evaluate the analyte partitioning effect, three different types of analytes were tested: anion mixtures, neutral mixtures and cation mixtures. Improved efficiency, resolution and selectivity were observed for both the anions and the polycyclic aromatic hydrocarbons mixtures when the capillary was coated with poly(aryl ether) dendritic polymer (G1). The cation electropherograms did not exhibit the desired resolution when the modified columns were used. This could be caused by a deficiency in the column preparation which results from a greater proportion of unmodified silanol surface area when compared to the modified surface area. The unmodified silanol wall causes two undesired effects for cations separation: (1) the strong EOF rapidly sweeps the cations to the outlet, which results in no resolution (2) the electrostatic interaction causes band broadening and peak tailing.

\subsection{Effects of $\mathrm{Si}-\mathrm{O}-\mathrm{Si}$ versus $\mathrm{Si}-\mathrm{O}-\mathrm{C}$ bonds}

We have examined the effect of $\mathrm{Si}-\mathrm{O}-\mathrm{Si}$ bonds versus $\mathrm{Si}-\mathrm{O}-\mathrm{C}$ bonds by comparing the electropherograms obtained with the benzyl triethoxysilane and the benzyl bromide coated capillary columns. A commercial anion test mixture of benzoic acid, phydroxybenzoic acid, and p-hydroxyphenylacetic acid was used to evaluate the effects of two different bonding mechanisms. The electropherograms demonstrate improved resolution and efficiency for $\mathrm{Si}-\mathrm{O}-\mathrm{Si}$ bonding versus $\mathrm{Si}-\mathrm{O}-\mathrm{C}$ bonding. These results support our multiple bonding hypotheses. The results also indicates that the formation of 
a bidentate or tridentate $\mathrm{Si}-\mathrm{O}-\mathrm{Si}$ attachment are highly favorable under the column coating procedure we developed. A resolution comparison between the $\mathrm{Si}-\mathrm{O}-\mathrm{Si}$ versus $\mathrm{Si}-\mathrm{O}-\mathrm{C}$ bonding was presented in Chapter 5, Table 5-2.

\subsection{Rationale for the Coating Attachment}

Based on our preliminary results, 3-(triethoxysilyl)propyl isocyanate was selected as the coupling agent to attach the neutral dendritic polymers on to the inside wall of a capillary. There are two approaches for attachment of the coating to the silica capillary wall. First, the linker may be attached to the wall and the appropriate alcohol coupled to the linker in-situ or the alcohol may be coupled to the linker followed by attachment of the linker to the capillary wall. We chose the latter approach since the coupling reaction can be controlled when it is outside the capillary and the integrity of the stationary phase is guaranteed. This approach permits complete structural characterization of the coating prior to its bonding to the inner wall of the capillary.

\subsection{Coating materials studied}

The primary materials selected, a G1 alcohol and a C18 alcohol, contain close to the same number of carbon atoms, but differ in their structures: one is a highly branched aromatic molecule, while the other is a linear alkane. Figures 6-1 and 6-2 depict the molecular structure differences between the two. The dendritic polymer covering the silanol wall will form a semi-spherical architecture, while the C18 chain will extend linearly outwards from the wall. This would result in a charge density difference on the capillary wall. In CE separations, the electroosmotic flow rate is governed by the 
potential drop across the surface double layer, which in turn is a function of the charge density on the capillary wall. ${ }^{5}$ As the charge density at the capillary surface is reduced, the electroosmotic flow is expected to be reduced. Figure 6-3 demonstrates the electroosmotic flow dependence on $\mathrm{pH}$, from a pH range of 4.0 to 9.3, for the $\mathrm{G} 1$ coated, C18 coated, and uncoated fused silica capillaries. An EOF suppression was observed for the G1 and C18 coated columns when the neutral marker thiourea was used. A lower EOF with the G1 coating versus the $\mathrm{C} 18$ coating was obtained over the entire $4-9.3 \mathrm{pH}$ range.

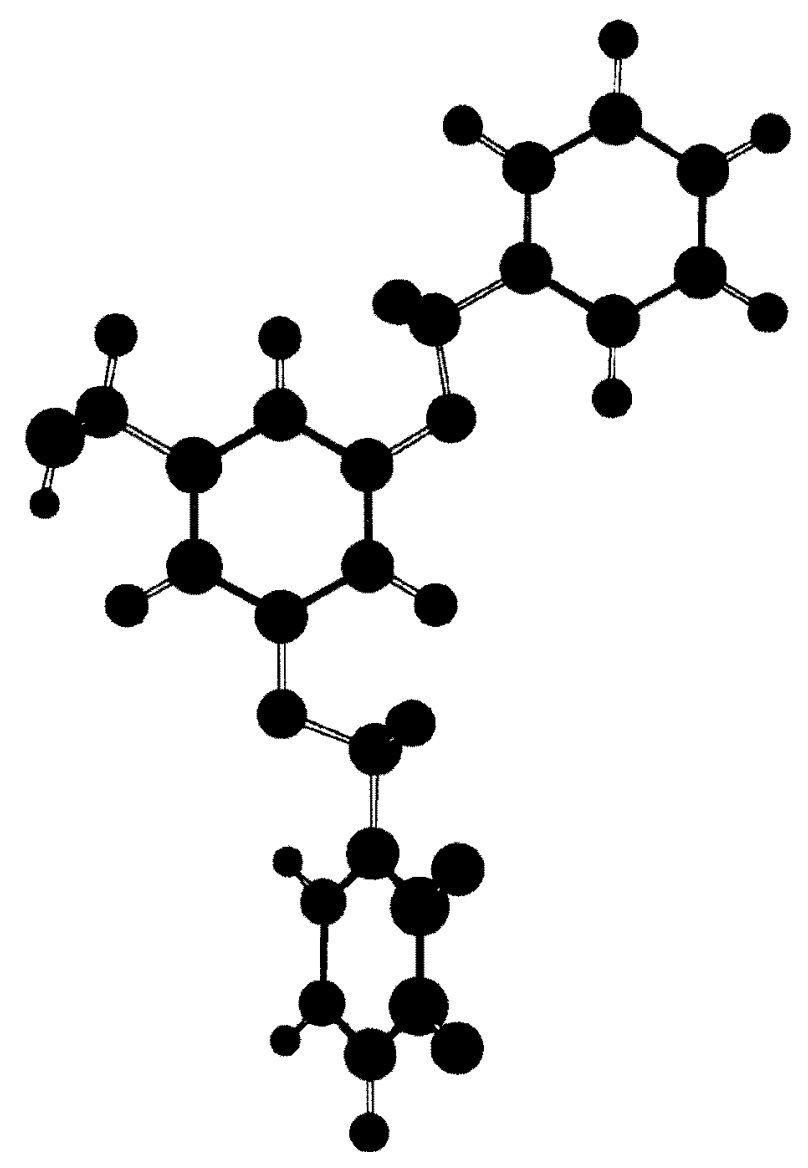

Figure 6-1. G1 alcohol molecular structure. 


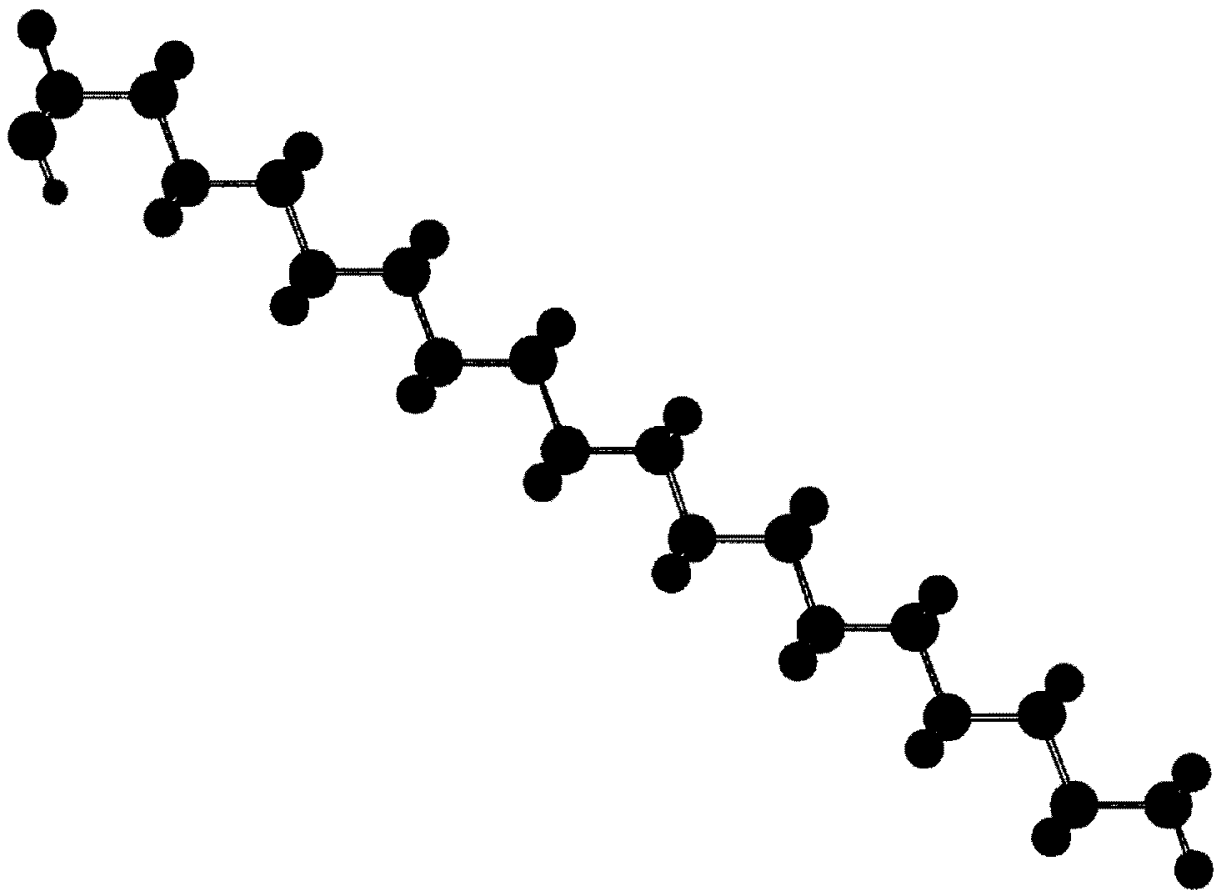

Figure 6-2. C18 alcohol molecular structure. 

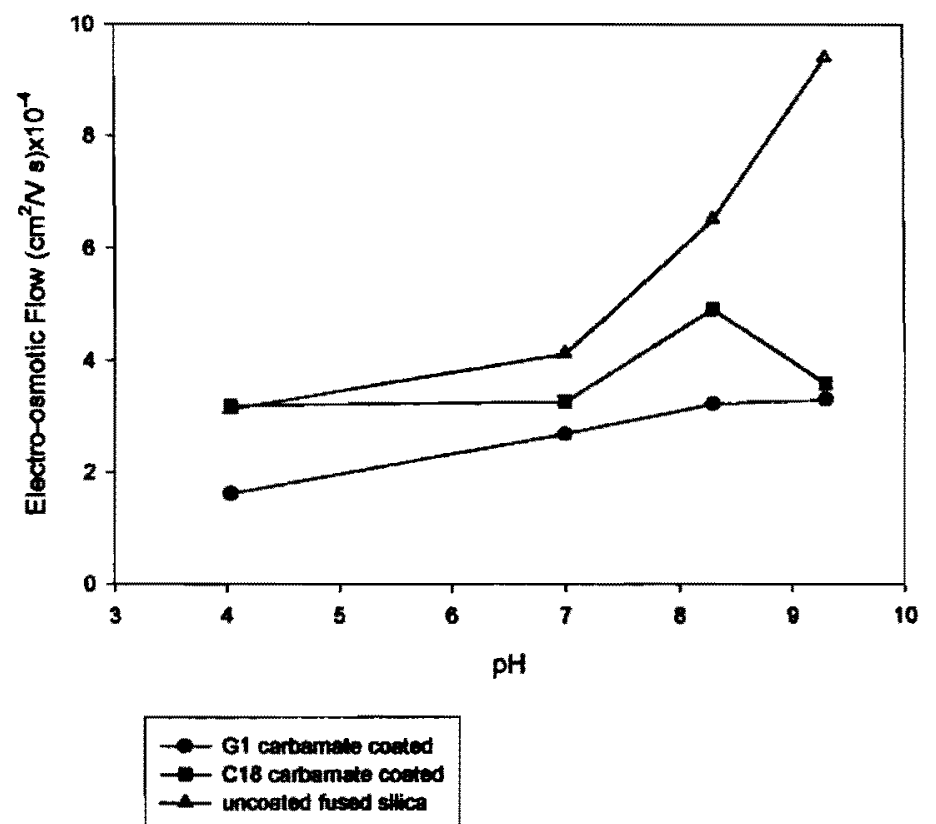

Figure 6-3. EOF dependence on $\mathrm{pH}$ and coating type: $\mathrm{G} 1$ carbamate coated, $\mathrm{C} 18$ carbamate coated, and uncoated silica capillary columns.

Based on the EOF results obtained from the G1 and C18 coated capillary columns, a lower EOF is predicted when the capillary column is coated with a generation 2 poly(aryl ether) dendrimer (G2) compared to the G1 coated column. The author examined a G2 coated column under the same CEC conditions that were performed with G1 and C18 coated columns. An EOF near zero was observed. This observation agrees with our hypothesis: higher generation dendrimers provide more surface area coverage than the lower generation dendrimers per molecule. As the dendrimer generation grows, the dendrimer backfolds. This effect assists in providing a uniform coverage on the silica surface, which results in a difference in the charge density on the capillary wall. The dendrimer wedge backfolding effect is illustrated in Figure 6-4. 


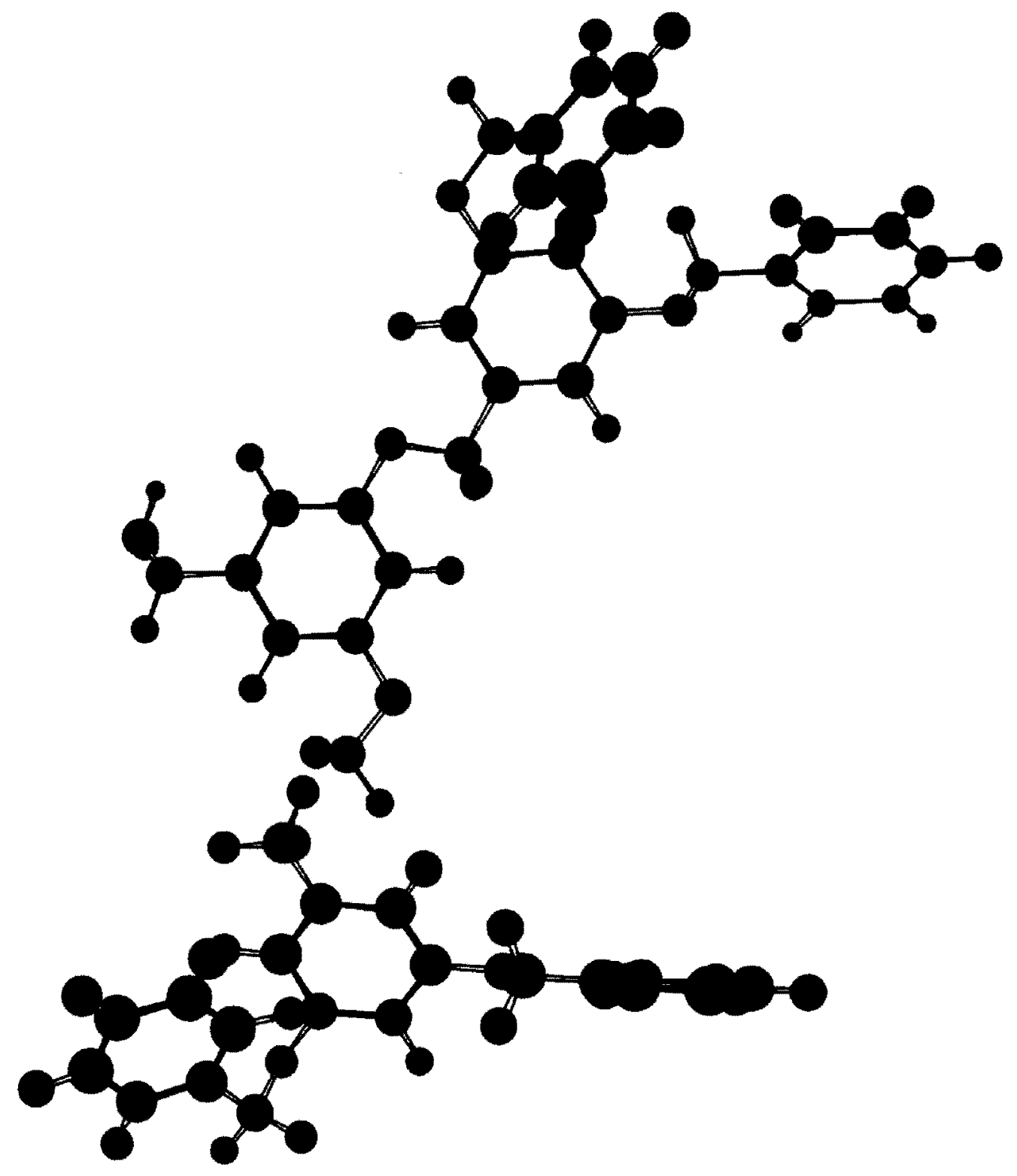

Figure 6-4. Generation 2 poly(aryl ether) dendrimer molecular structure. 
Another surface modification technique, which has been studied by separation scientists, is to coat the inner surface of a capillary with materials that will easily be protonated under acidic conditions, such as polyethyleneimine. 6 With this modification, the net charge of the inner wall is positive which results in a change in EOF direction. In this report, 4-pyridyl carbinol was examined as a test material. At $\mathrm{pH} 4.0$, the electroosmotic flow observed is $1.10 \times 10^{-4} \mathrm{~cm}^{2} \mathrm{v}^{-1} \mathrm{~s}^{-1}$ which is a $30 \%$ reduction in EOF compared to the $\mathrm{G} 1$ coatings and a $65 \%$ reduction in EOF compared to the $\mathrm{C} 18$ coatings using the same conditions.

\subsection{Capillary column performance}

\subsubsection{Evalution of coated capillaries with polyaromatic compounds. Neutral species} can not be separated by traditional capillary electrophoresis. In 1984, Tarabe et al developed the MECC technique specifically to solve this problem which is inherent to CE. In MECC, surfactants such as SDS or CTAB are added to the buffer electrolyte as a so-called pseudo-stationary phase. To date, there are still certain limitations to this technique: (1) suitable micelles, (2) solubility and stability of the micelles in aqueousorganic solvent mixtures. Research on new micelles, zwitterions, and other chemicals that might lead to improved selectivity performance, have spawned more interest. The first paper to examine dendrimers as a pseudo-stationary phase for MECC was published by Tarabe's group in 1992. In their study, alkylated polyamidoamine starburst dendrimers (PAMAM-SBDs), which are commercially available, were compared with SDS. They reported the SBD additive showed little selectivity for alkyl groups but a clear preference for aromatic compounds was found. Improved selectivity for rigid, 
planar polynuclear aromatic hydrocarbons was specifically observed with their investigations. We made a similar observation with our G1 coated capillary column using the OT-CEC technique. The separation of toluene, naphthalene and phenanthrene was used to demonstrate the resolution and selectivity provided by the G1 coated column.

One of the most interesting observations in our research is the temperature effect on the resolution and peak shape. Due to Joule heating, the CEC column temperature is generally controlled at $20^{\circ} \mathrm{C}$ to $25^{\circ} \mathrm{C}$ with air or liquid cooling. We investigated the separation of the polynuclear aromatic compounds at several different temperatures, $30^{\circ} \mathrm{C}, 25^{\circ} \mathrm{C}, 20^{\circ} \mathrm{C}$, and $15^{\circ} \mathrm{C}$. We found the best resolution and peak shape at $15^{\circ} \mathrm{C}$, in a pH 6.0, 5 mM MES buffer solution and 30\% methanol as the organic modifier. These results could be explained by the basic liquid chromatography separation mechanism- a partitioning effect. The separation factor, $\alpha$ is determined by thermodynamic factors (i.e. the relative retention is affected by the distribution coefficients which are a function of the temperature). Our observation suggests that at the lower temperature, the G1 stationary phase undergoes a phase transition which aligns with the polymer's molecular structure conformation. This structural rearrangement favors to $\pi-\pi$ interaction as one of the molecular interaction in chiral separations. In our case, the molecular interactions of naphthalene and phenanthrene with the stationary phase are favored as the molecules of the stationary phases align in an orderly conformation. Figure 6-5 shows the polynuclear aromatic hydrocarbons electropherograms obtained with a Gl coated column at different temperatures. 


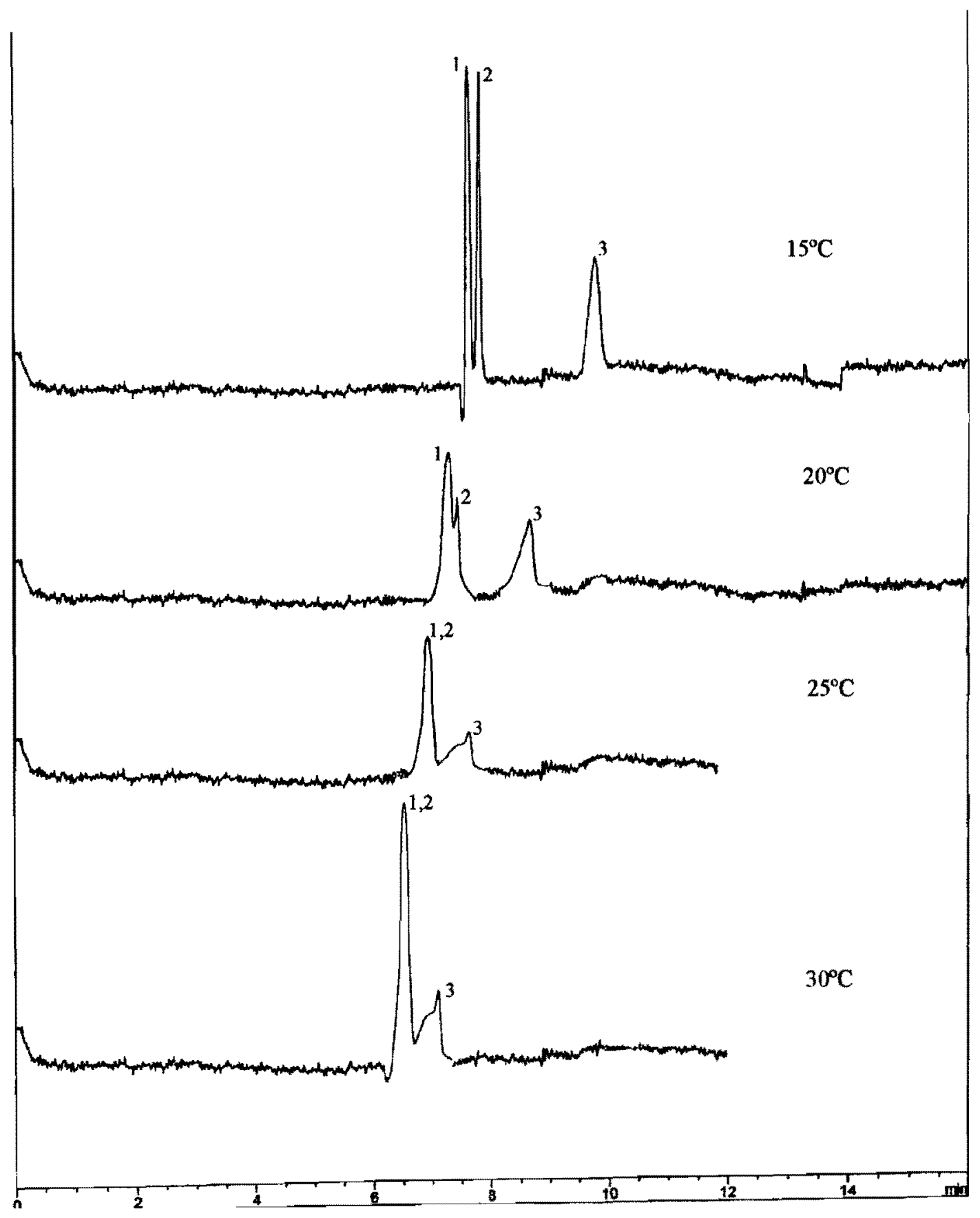

Figure 6-5. The separation of (1) toluene, (2) naphthalene, and (3) phenanthrene obtained at different temperatures with a G1 coated column. 
The sample electropherograms from Tarabe's report showing the comparison results of SDS and SBD(x) are shown in Figure 6-6 and 6-7.
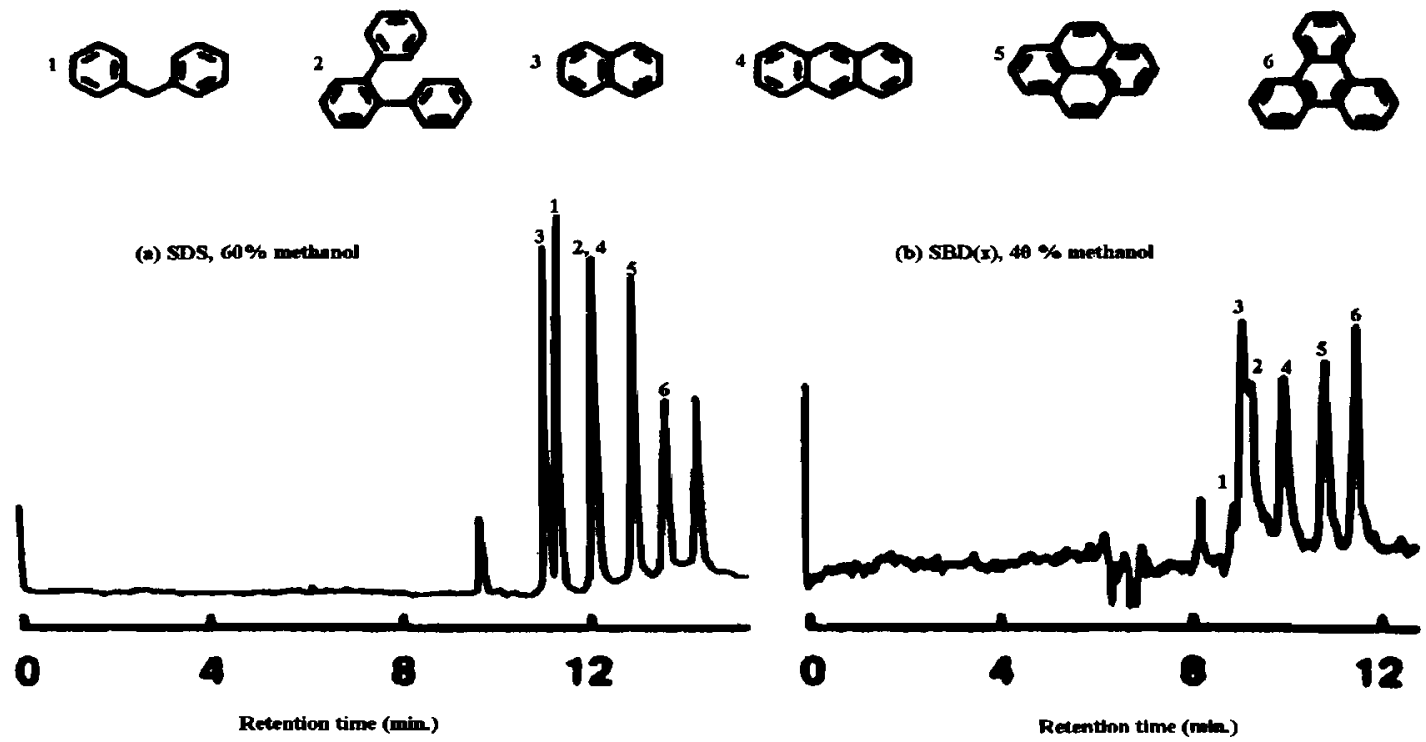

Figure 6-6. Electropherograms demonstrating the structural selectivity provided by PAMAM. ${ }^{7}$


Figure 6-7. Electropherograms showing the difference in selectivity between SDSMECC and SBD-EKC. 7 
6.5.2 Evaluation of coated capillaries with basic amine substances. The separations of basic amine compounds are difficult to achieve with the traditional CE methods. We evaluated the performance of our capillary columns, which were coated with G1 and C18 coated, for the separation of a mixture of phenyl ethylamine, 2,6-difluoroaniline and 2'fluoroacetanilide. At $\mathrm{pH} 4.0$, phenyl ethylamine migrated as a cation, and was separated from the other two amines. However, the G1 and C18 coatings did not provide good selectivity for 2,6-difluoroaniline and 2'-fluoroacetanilide. These amines remain as neutral species due to their low pKa value and this results in poor selectivity. The pKa of 2,6-difluoroaniline was estimated to be $\sim 2$ to 3 . 2'-fluoroacetanilide is classified as a neutral compound in general.

\subsubsection{Evaluation of coated capillaries with basic proteins. Like the basic amine} compounds, the adsorption of positively charged proteins onto the walls of fused-silica capillaries is a problem in CE. A G1 coated capillary column's performance was evaluated for the separation of a commercial protein test mixture which consisted of lysozyme, cytochrome $\mathrm{C}$ and ribonuclease $\mathrm{A}$. Electropherograms were obtained using a pH 6 MES.buffer, a pH 7.0 phosphate buffer and pH 8.3 TRIS buffer. Problems related to detection sensitivity and sample injection were noticed and experimental conditions were difficult to control.

6.5.4 Evaluation of coated capillaries with anions. In this study, some adverse effects were observed when an anion test mixture was examined at $\mathrm{pH}=9.30$. This is most likely due to the stability of the carbamate bond which links the coating material to 
the coupling agent, 3-(triethoxysilyl)propyl isocyanate. Carbamates are known to be less stable below pH 4.0 and above $\mathrm{pH} 8.0$.

6.5.5 Evaluation of the selectivity effect on structural isomers. Positive results were observed for the separation of the regioisomers of epinephrine and pyrrolopyridine when G1 and 4-pyridyl carbinol coated capillary columns.

Unexpected results were obtained for epinephrine. Two peaks were observed for this amine when the electropherograms were obtained with the G1 and 4-pyridyl carbinol coated capillary columns, Figure 5-21. The observation of two epinephrine peaks is unusual since this optically active compound (i.e. chiral) should give a single response in an achiral environment. To better understand this result, further examination of the epinephrine separation was made using the G1 coated capillary column with buffers at $\mathrm{pH} 4,7,8.3$, and 9.3, Figure 5-22. The two peaks for epinephrine were observed only with a pH 4.0 buffer. Since the epinephrine purity is only $95 \%$, as stated by Aldrich, an explanation to this observations is due to three possible compounds: nordefrin metanephrine and. normetanephrine. Nordefrin is a structural isomer of epinephrine and has a primary amine group. It is very probable that the pKa of epinephrine is greater than 4.0 and the $\mathrm{pKa}$ of nordefrin is less than 4.0 . In a $\mathrm{pH} 4.0$ buffer, the epinephrine is protonated and it migrates as a cation, the nordefrin remains as a neutral amine and therefore migrates more slowly. This hypothesis agrees with the results obtained with the 4-pyridyl coated column. If the EOF direction is reversed, the migration order is reversed. On the other hand, metanephrine and. normetanephrine are both naturally occurring derivatives of epinephrine, which are found in urine and in certain tissues. If 
the additional peak found is due to one of the naturally occurring derivatives as an impurity in the epinephrine material, the pKa of the impurity compound must be less than 4.0. It is unlikely the pKa value of metanephrine or normetanephrine is less than 4.0 based on their molecular structure. The molecular structures of epinephrine and the three possible impurities are shown in Figure 6-8.<smiles>CNC[C@H](O)c1ccc(O)c(O)c1</smiles>

Epinephrene<smiles>CNC[C@H](O)c1ccc(O)c(OC)c1</smiles>

Metanephrine<smiles>CC(N)[C@H](O)c1ccc(O)c(O)c1</smiles>

Nordefrin<smiles>COc1cc([C@@H](O)C(C)N)ccc1O</smiles>

Normetanephrine

Figure 6-8. The molecular structures of the epinephrine and the three possible impurities.

Both regio-isomers of pyrrolopyridine, the $(3,4 b)$ and $(2,3 c)$, were separated with an uncoated, C18 coated, and G1 coated capillary columns. The separation factors $(\alpha)$ of each column were calculated and they are listed in Table 6-1. The G1 coated column provided the best selectivity and this is clearly demonstrated by the separation factors. 
Table 6-1. Pyrrolopyridine separation factors obtained from G1, C18 coated and uncoated columns

\begin{tabular}{|c|c|c|c|}
\hline & \multicolumn{3}{|c|}{ Separation Factor } \\
\hline & G1 & C18 & Uncoated \\
\hline \hline$(2,3 c) /(3,4 b)$ & 1.21 & 1.08 & 1.09 \\
\hline
\end{tabular}

The separation factor $(\alpha)$ is the chromatographic parameter that is directly related to the selectivity of the phase system. In practice, it can be defined as the relative retention of two peaks:

$$
\alpha_{2,1}=\mathrm{t}_{2}^{\prime} / \mathrm{t}^{\prime}{ }_{1}
$$

where, $t^{\prime}{ }_{2}$ is the relative retention of peak 2 , and $t^{\prime}{ }_{1}$ is the relative retention of peak 1 .

\subsection{Conclusion}

In this investigation, a new class of macromolecules has been investigated as bonded stationary phases for open tubular capillary electrochromatography (OT-CEC). A reliable method was developed to prepare the stationary phase for OT-CEC. It has been demonstrated that the highly branched polymer stationary phases provide unique features compared to linear polymers. The controlled synthesis and the molecular architecture allowed the rational variation of the coating materials. This was a pioneer study to explore the utility of the poly(aryl ether) dendrimers as OT-CEC coating materials. The results obtained from the generation 1 poly(aryl ether) dendrimer are very promising. The poly(aryl ether) dendrimers coatings were found to have several unique 
features: (1) suppression of electroosmotic flow, (2) solute partitioning effect, and (3) improved resolution of CEC. Attachment using 3-(triethoxysilyl)propyl isocyanate as an anchoring agent gave better results than the direct connection of the benzylic ether, most likely due to greater stability of the tridentate $\mathrm{Si}-\mathrm{O}-\mathrm{Si}$ bonds. However, the carbamate linker is restricted to a pH region of $4-9$.

The flexibility of adding different substituents onto the core structure would lead to a variety of applications for dendrimer coatings. With the increased demand and interest in chiral separations, ${ }^{8-11}$ a dendrimer targeted for the use of stereoselective separations could be designed. The ability to generate "tailored" coatings for specific separations makes these materials very promising for CEC and/or for chromatography applications and promises to be a fertile area of research. 


\subsection{Reference for chapter 6}

1) Bruin, G. J. M.; Tock, P. P. H.; Kraak, J. C.; Poppe, H. J. Chromatogr. 1990, 517, 557-572.

2) Onuska, F.; Comba, M. E.; Bistricki, T.; Wilkinson, R. T. J. Chromatogr. 1977, 142, 117.

3) Pesek, J. J.; Matyska, M. J. J. Chromatogr. A 1996, 736, 313.

4) Tock, P. P. H.; Boshoven, C.; Poppe, H.; Kraak, J. C. J. Chromatogr. 1989, 477, 95.

5) Pretorius, V.; Hopkins, B. J.; Schieke, J. D. J. Chromatogr. 1974, 99, 23.

6) Towns, J. K.; Regnier, F. E. J. Chromatogr., 1990, 516, 1126.

7) Tanaka, N.; Fukutome, T.; Tanigawa, T.; Hosoya, K.; Kimata, K.; Araki, T.; and Unger, K. K. J. of Chromatog. A 1995, 699, 331-341.

8) Schurig, V.; Mayer, S. J. of Liq. Chroma. 1993, 16, 915.

9) Frechet, J. M. J.; Liu, Y.; Sevc, F. Anal. Chem. 1997, 69, 61.

10) Warner, I. M.; Agnew-Heard, K. A.; Pena, M. S.; Shamsi, S. A. Anal. Chem. $1997,69,958$.

11) Zare, R. N.; Lelievre, F.; Yan, C.; Gareil, P. J. Chromatogr. A, 1996, 723, 145. 